\title{
Design of sandwich-like complexes based on the tetracoordinate planar carbon unit $\mathrm{CAl}_{4}{ }^{2-}$
}

\section{Supporting Information.}

Li-ming Yang, Yi-hong Ding*, Chia-chung Sun

State Key Laboratory of Theoretical and Computational Chemistry, Institute of Theoretical Chemistry, Jilin University, Changchun 130023, People's Republic of China

\section{Full citations for ref 9f:}

9.(f) Gaussian03 (RevisionA.1), M. J. Frisch, G. W. Trucks, H. B. Schlegel, G. E. Scuseria, M. A. Robb, J. R. Cheeseman, J. A. Montgomery, Jr., T. Vreven, K. N. Kudin, J. C. Burant, J. M. Millam, S. S. Iyengar, J. Tomasi, V. Barone, B. Mennucci, M. Cossi, G. Scalmani, N. Rega, G. A. Petersson, H. Nakatsuji, M. Hada, M. Ehara, K. Toyota, R. Fukuda, J. Hasegawa, M. Ishida, T. Nakajima, Y. Honda,O. Kitao, H. Nakai, M. Klene, X. Li, J. E. Knox, H. P. Hratchian, J. B. Cross, C. Adamo, J. Jaramillo, R. Gomperts, R. E. Stratmann, O. Yazyev, A. J. Austin, R. Cammi, C. Pomelli, J. W. Ochterski, P. Y. Ayala, K. Morokuma, G. A. Voth, P. Salvador, J. J. Dannenberg, V. G. Zakrzewski, S. Dapprich, A. D. Daniels, M. C. Strain, O. Farkas, D. K. Malick, A. D. Rabuck, K. Raghavachari, J. B. Foresman, J. V. Ortiz, Q. Cui, A. G. Baboul, S. Clifford, J. Cioslowski, B. B. Stefanov, G. Liu, A. Liashenko, P. Piskorz, I. Komaromi, R. L. Martin, D. J. Fox, T. Keith, M. A. Al-Laham, C. Y. Peng, A. Nanayakkara, M. Challacombe, P. M.W. Gill, B. Johnson, W. Chen, M.W. Wong, C. Gonzalez, J. A. Pople, Gaussian, Inc., Pittsburgh, PA, 2003.

Additional references on TPC before year 1995.

S1. Krogh-Jesperson, M. B.; Cremer, D.; Poppinger, D.; Pople, J. A.; Schleyer, P. v. R.; Chandrasekhar, J. J. Am. Chem. Soc. 1979, 101, 4843.

S2. Wurthwein, E.-U.; Schleyer, P.v.R. Angew. Chem. Int. Ed. 1979, 18, 553.

S3. Krogh-Jesperson, M. B.; Chandrasekhar, J.; Wurthwein, E. U.; Collins, J. B.; Schleyer, P. v. R. J. Am. Chem. Soc. 1980, 102, 2263.

S4. Chadrasekhar, J.; Wurthwein, E. U.; Schleyer, P. v. R. Tetrahedron 1981, 37, 921.

S5. Wurthwein, E. U.; Chadrasekhar, J.; Jemmis, E. D.; Schleyer, P. v. R. Tetrahedron Lett. 1981, 22, 843. 
S6. Chandrasekhar, J.; Schleyer, P. v. R. J. Chem. Soc., Chem. Commun. 1981, 260.

S7. Schleyer, P. v. R. Pure Appl. Chem. 1983, 55, 355;

S8. Schleyer, P. v. R. Pure Appl. Chem. 1984, 56, 151.

S9. Schleyer, P. v. R.; Clark, T.; Kos, A. J.; Spitznagel, G. W.; Rohde, C.; Arad, D.; Houk, K. N.; Rondan, N. G. J. Am. Chem. Soc. 1984, 106, 6467

S10. Setzer, W. N.; Schleyer, P. v. R. Adv. Organomet. Chem. 1985, 24, 353.

S11. Sapse, A.-M.; Raghavachari, K.; Schleyer, P. v. R.; Kaufmann, E. J. Am. Chem. Soc. 1985, 107, 6483.

S12. Schleyer, P. v. R.; Kaufmann, E.; Spitznagel, G. W.; Janoschek, R.; Winkelhofer, G. Organometallics 1986, 5, 79.

S13. Bauer, W.; Winchester, W. R.; Schleyer, P. v. R. Organometallics 1987, 6, 2371.

S14. Geissler, M.; Kopf, J.; Schubert, B.; Weiss, E.; Neugebauer, W.; Schleyer. P. v. R. Angew. Chem. 1987, 99, 569; Angew. Chem., Int. Ed. Engl. 1987, 26, 587.

S15. Kaufmann, E.; Raghavachari, K.; Reed, A. E.; Schleyer, P. v. R. Organometallics 1988, 7, 1597.

S16. Bauer, W.; Klusener, P. A. A.; Harder, S.; Kanters, J. A.; Duisenberg, A. J. M.; Brandsma, L.; Schleyer, P. v. R. Organometallics 1988, 7, 552.

S17. Harder, S.; Boersma, J.; Brandsma, L.; van Heteren A.; Kanters, J. A.; Bauer, W.; Schleyer, P. v. R. J. Am. Chem. Soc. 1988, 110, 7802.

S18. Bauer, W; Schleyer, P. v. R. J. Am. Chem. Soc. 1989, 111, 7191

S19. Gregory, K.; Schleyer, P. v. R.; Snaith, R. Adv. Inorg. Chem. 1991, 37, 47.

S20. Lambert, C.; Schleyer, P. v. R. Angew. Chem. 1994, 106, 1187; Angew. Chem., Int. Ed. Engl. 1994, 33, 1129.

S21. Preliminary communication: Sorger, K.; Schleyer, P. v. R.; Stalke, D. J. Chem. Soc, Chem. Commun. 1995, 2279.

S22. Wiberg, K.; Hiatt, J. E.; Burgmaier, G. Tetrahedron Lett. 1968, 5855.

S23. Wiberg, K. B.; Elison, G. B.; Wendolski, J. J. J. Am. Chem. Soc. 1976, 98, 1212. S22.

Laidig, W. D.; Schaefer, H. F., III J. Am. Chem. Soc. 1978, 100, 5972.

S24. Wiberg, K. B.; Odonnell, J. Am. Chem. Soc. 1979, 101, 6660.

S25. Bohm, M. C.; Gleiter, R.; Schlang, P. Tetrahedron Lett. 1979, 2675. 
S26. Keese, R.; Pfenninger, A.; Roesle, A. Helv. Chim. Acta 1979, 62, 326.

S27. Wiberg, K. B.; Olli, L. K.; Golembeski, N.; Adams, R. D. J. Am. Chem. Soc. 1980, 102, 7467.

S28. Schori, H.; Patil, B. B.; Keese, R. Tetrahedron 1981, 37, 4457.

S29. Wiberg, K. B.; Wendoloski, J. J. J. Am. Chem. Soc. 1982, 104, 5679.

S30. Cotton, F. A.; Lewis, G. F.; Mott, G. N. Inorg. Chem. 1983, 22, 560.

S31. Bachrach, S. M.; Streitweiser, A., Jr. J. Am. Chem. Soc. 1984, 106, 5818.

S32. Frenking, G. Chem. Phys. Lett. 1984, 111, 529.

S33. Wiberg, K. B. Tetrahedron Lett. 1985, 26, 5967.

S34. Dodziuk, H. Bull. Chem. Soc. Jpn. 1987, 60, 3775.

S35. Glukhovtsev, M. N.; Simkin, B. Ya.; Minkin, V. I. J. Org. Chem. USSR 1990, 26, 1933.

S36. Dodziuk, H. J. Mol. Struct. 1990, 239, 167.

S37. Glukhovtsev, M. N.; Simkin, B. Ya.; Minkin, V. I. J. Org. Chem. USSR 1991, 27, 1.

S38. Erker, G.; Albrecht, M.; Kruger, C.; Wener, S. Organometallics 1991, 10, 3791.

S39. Thommen, M.; Gerber, P.; Keese, R. Chimia 1991, 45, 21.

S40. Luef, W.; Keese, R. J. Mol. Struct. (THEOCHEM) 1992, 257, 353.

S41. McGrath, M. P.; Schaefer, H. F., III; Radom, L. J. Org. Chem. 1992, 57, 4847.

S42. Albrecht, M.; Erker, G.; Notle, M.; Kruger, C. J. Organomet. Chem. 1992, 427, C21.

S43. Erker, G.; Albrecht, M.; Wener, S.; Notle, M.; Wener, S.; Binger, P.; Langhauser, F. Organometallics 1992, 11, 3517.

S44. Erker, G.; Albrecht, M.; Kruger, C.; Wener, S. J. Am. Chem. Soc. 1992, 114, 8531.

S45. Gleiter, R.; Hyla-Kryspin, I.; Niu, S.; Erker, G. Angew. Chem., Int. Ed. Engl. 1993, 32, 754.

S46. McGrath, M. P.; Radom, L. J. Am. Chem. Soc. 1993, 115, 3320.

S47. Poumbga, C. N.; Bernar, M.; Hyla-Kryspin, I. J. Am. Chem. Soc. 1994, 116, 8259.

S48.Glukhovtsev, M. N.; Pross. A.; Radom, L. J. Am. Chem. Soc. 1994, 116, 5961.

S49. Nakajima, A.; Taguwa, T.; Nakao, K.; Hoshino, K.; Iwata, S.; Kaya, K. J. Chem. Phys. 1995, 102, 660. 
Note1. An imaginary frequency indicates the existence of a vibrational mode that is dynamically unstable and leads to a more stable structure. Transition states of a chemical reaction are saddle points exhibiting only one imaginary frequency. Saddle points with more dynamical systems with sufficiently high vibrational energy but are generally not of chemical significance. We applied the SCF convergence of $10^{-6}$ hartree designation for the energy convergence in optimized geometries at the B3LYP/6-31+G(d) due to thundering gigantic workload.

(i): means that the isomer is not a minimum point.

1. The calculated properties of ${ }^{1} \mathrm{CAl}_{4} \mathrm{LiCAl}_{4}{ }^{3-}$

The potential energy surface of $\mathrm{CAl}_{4} \mathrm{LiCAl}_{4}{ }^{3-}$ and many other isomers of $\mathrm{CAl}_{4} \mathrm{LiCAl}_{4}{ }^{3-}$

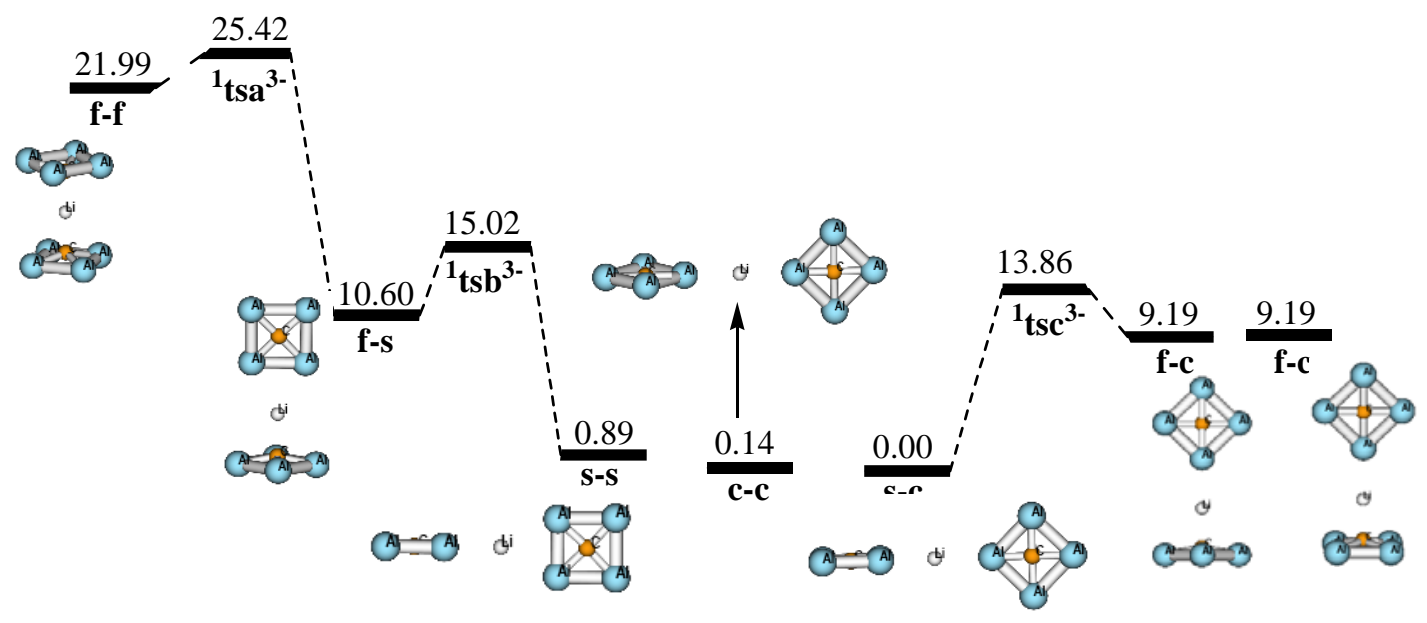

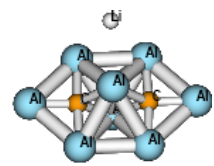

18.13

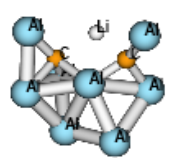

31.67

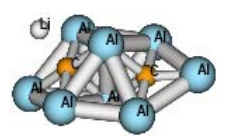

20.41

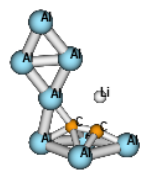

32.11

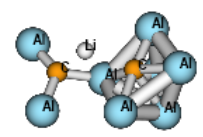

22.44

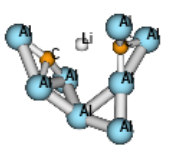

38.76

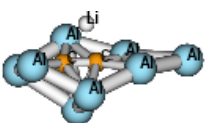

24.07

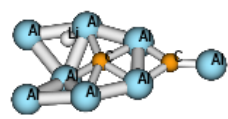

42.19

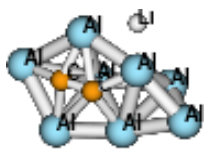

26.84

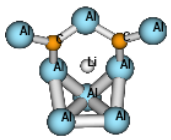

42.37 


\section{The calculated properties of ${ }^{1} \mathrm{CAl}_{4} \mathrm{NaCAl}_{4}{ }^{3-}$}

The potential energy surface of $\mathrm{CAl}_{4} \mathrm{NaCAl}_{4}{ }^{3-}$ and many other isomers of $\mathrm{CAl}_{4} \mathrm{NaCAl}_{4}{ }^{3-}$

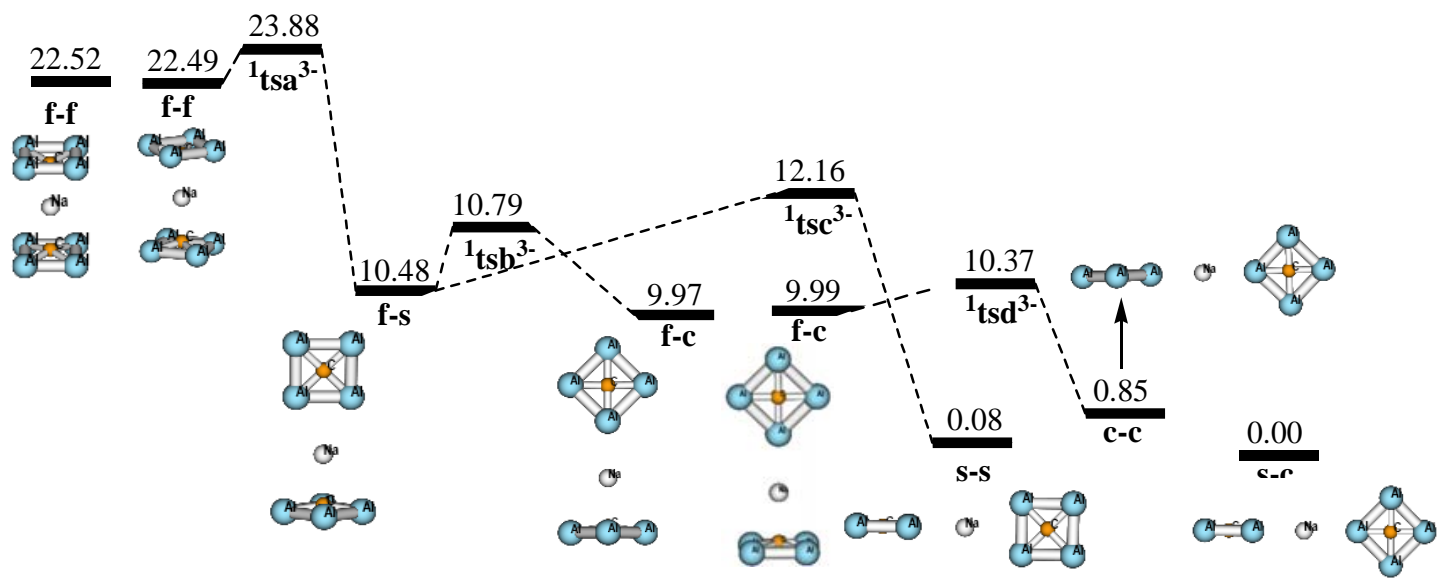

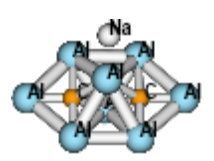

17.55

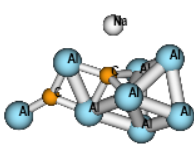

35.21

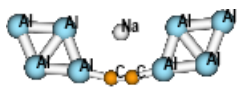

52.08

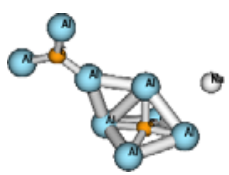

19.41

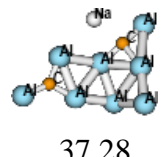

37.28

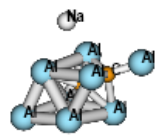

22.92

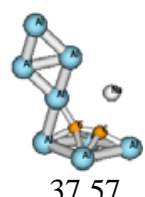

37.57

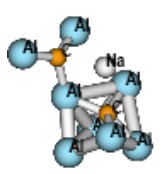

26.04

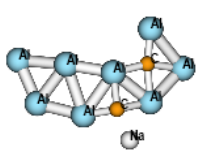

41.14
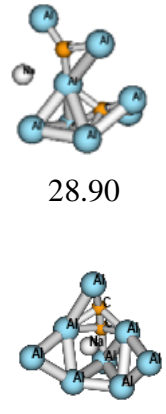

42.24 


\section{The calculated properties of ${ }^{1} \mathrm{CAl}_{4} \mathrm{KCAl}_{4}{ }^{3-}$}

The potential energy surface of $\mathrm{CAl}_{4} \mathrm{KCAl}_{4}{ }^{3-}$ and many other isomers of $\mathrm{CAl}_{4} \mathrm{KCAl}_{4}{ }^{3-}$

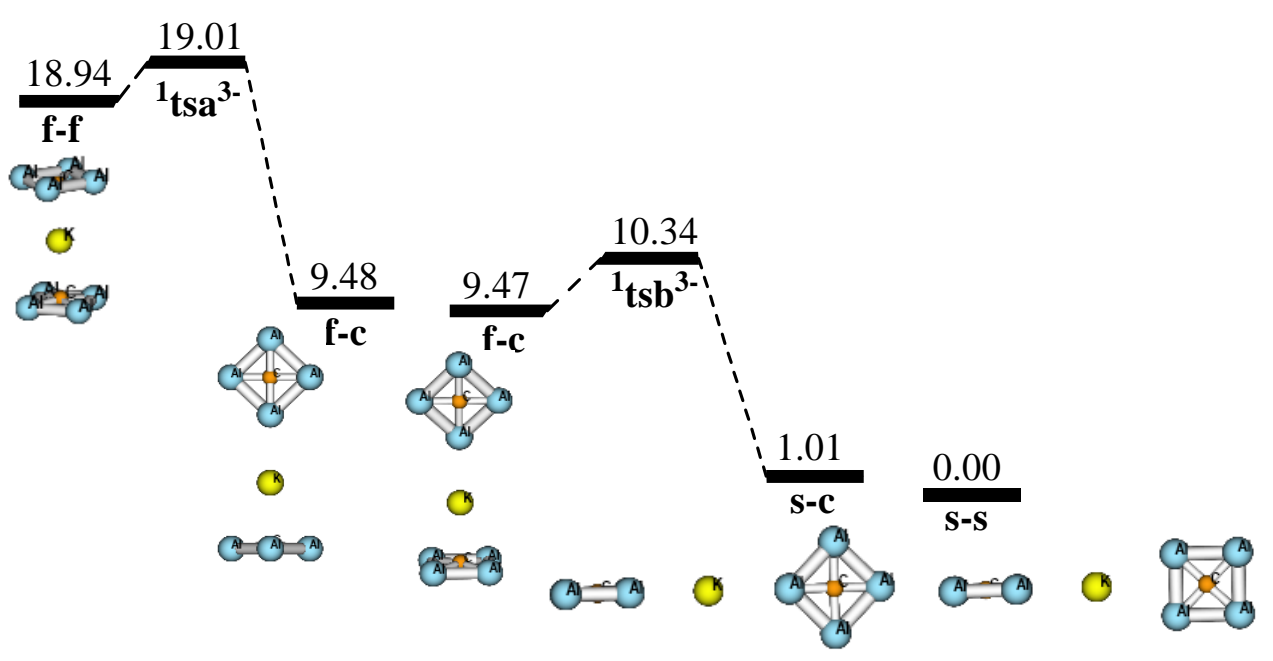

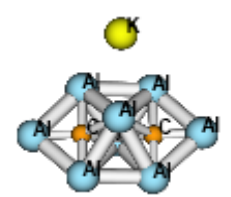

4.35

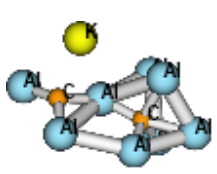

13.46

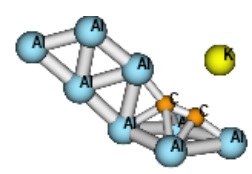

21.05

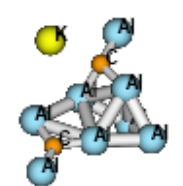

24.69

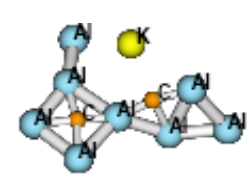

47.05 
4. The calculated properties of ${ }^{1} \mathrm{CAl}_{4} \mathrm{BeCAl}_{4}{ }^{2-}$

The relative energy of isomers of $\mathrm{CAl}_{4} \mathrm{KCAl}_{4}{ }^{3-}$
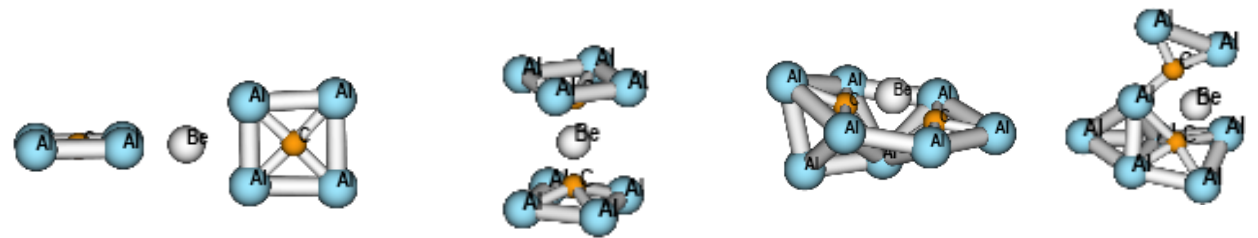

s-s(0.00)

$$
\mathbf{f - f}(8.17)
$$

$-12.63$

$-5.75$
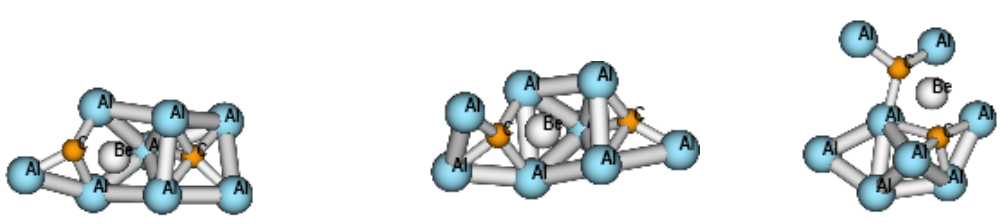

5.38

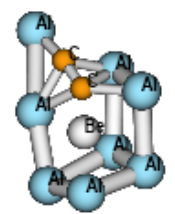

$-2.28$

$$
0.51
$$
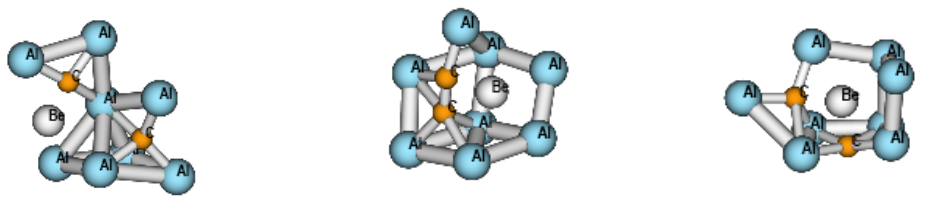

7.74

12.31

12.37

15.68

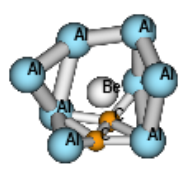

19.24
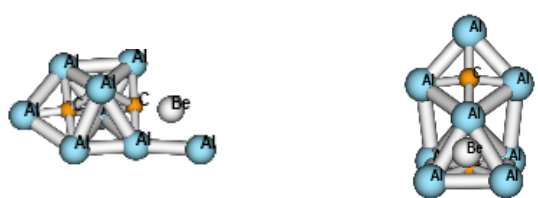

25.50
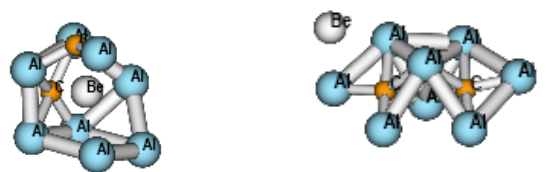

31.08

42.04 
5. The calculated properties of ${ }^{1} \mathrm{CAl}_{4} \mathrm{MgCAl}_{4}{ }^{2-}$

The relative energy of isomers of $\mathrm{CAl}_{4} \mathrm{MgCAl}_{4}{ }^{2-}$
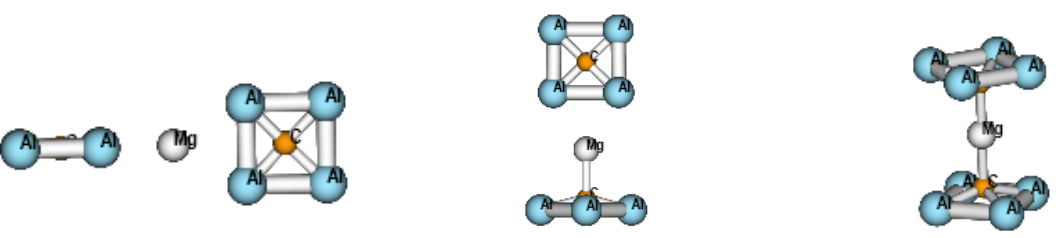

$$
\text { s-s(0.00) }
$$

f-s(14.49)

$$
\mathbf{f - f}(30.70)
$$

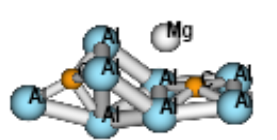

12.37

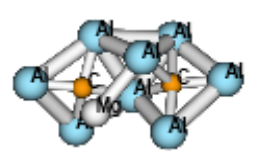

21.50

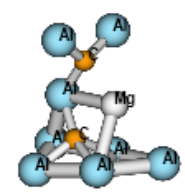

24.01

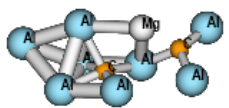

25.58

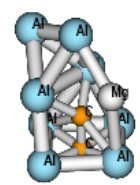

27.18

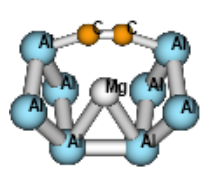

36.54

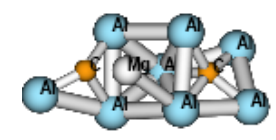

15.93

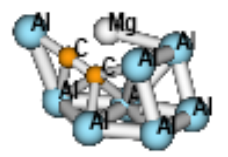

22.48

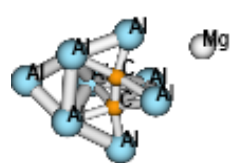

24.16
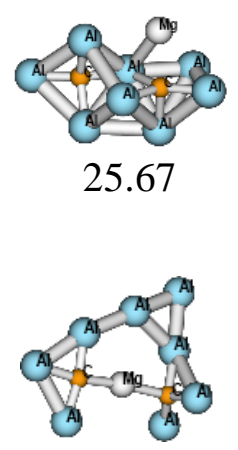

27.87

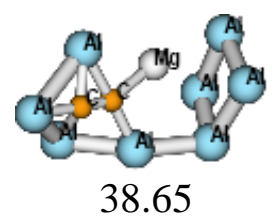

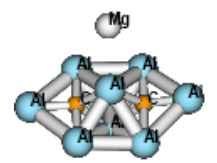

17.24

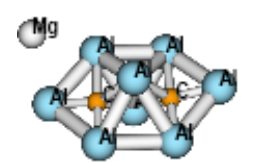

22.71

Mg

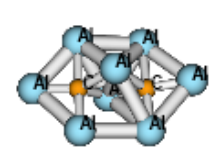

24.65
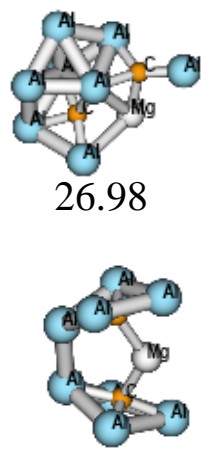

31.38

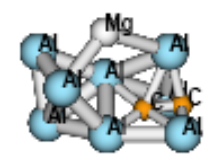

40.81

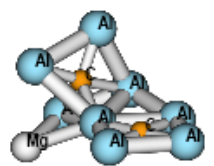

20.84

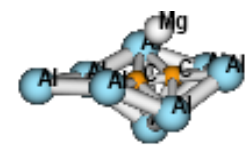

22.80

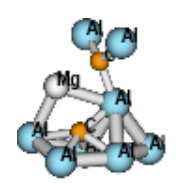

24.94

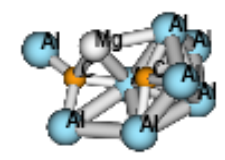

27.10

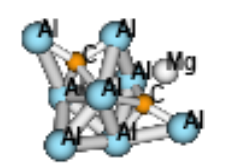

31.60

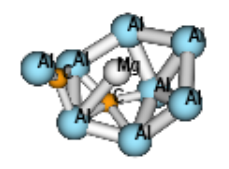

46.08 
6. The calculated properties of ${ }^{1} \mathrm{CAl}_{4} \mathrm{CaCAl}_{4}{ }^{2-}$

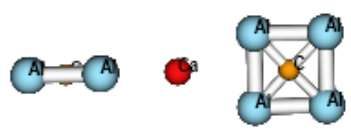

$\mathbf{s - s}(0.00)$

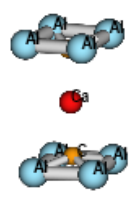

26.99(i)
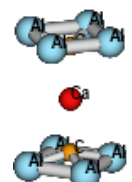

26.76(i)

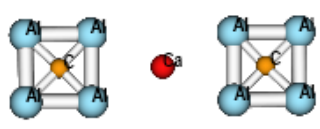

2.46(i)

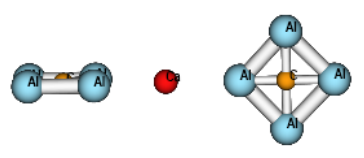

10.58(i)

(i): means that the isomer is not an minimum point.

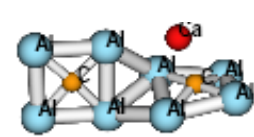

9.76

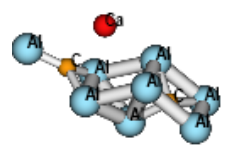

15.68

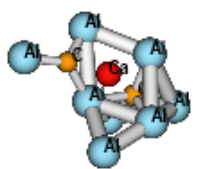

27.78

o

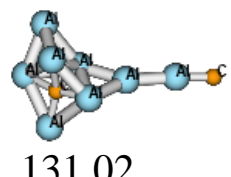

131.02

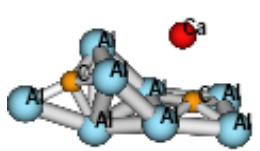

12.83

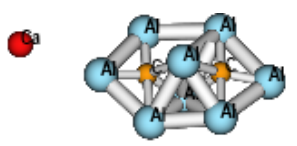

24.44

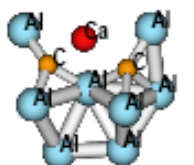

33.26

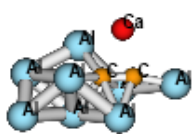

13.70

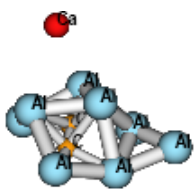

25.34

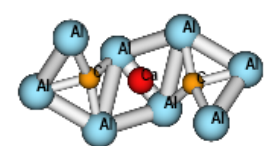

37.15

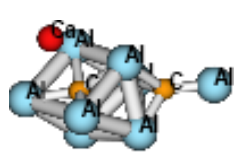

15.20
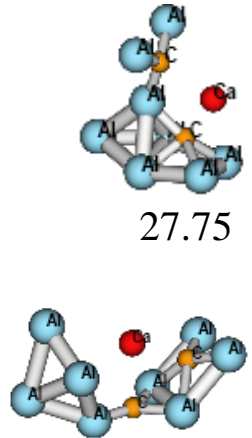

56.73 
7. The calculated properties of ${ }^{1} \mathrm{C}_{2} \mathrm{Al}_{8} \mathrm{Li}_{4}$
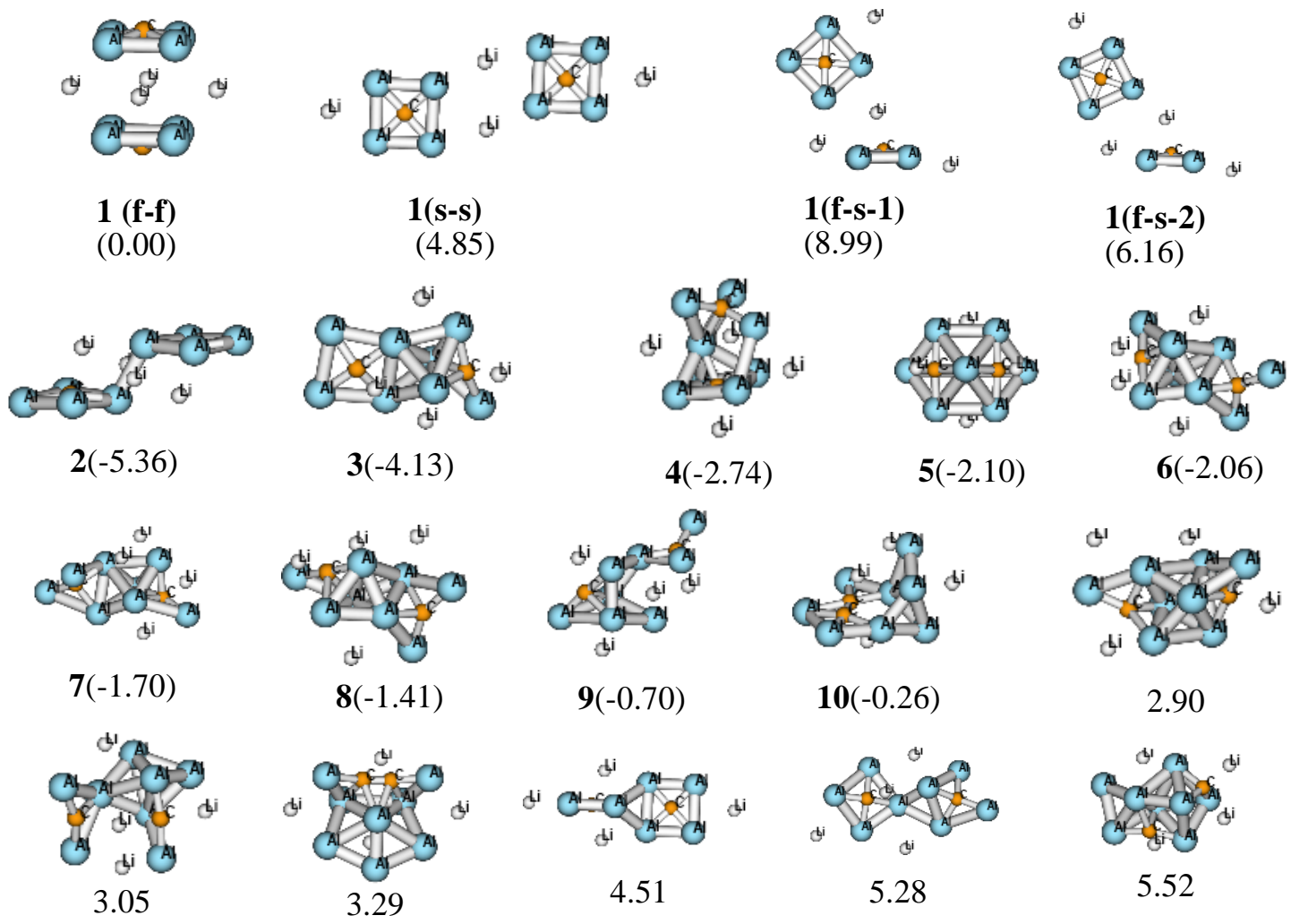

4.51

5.28

5.52
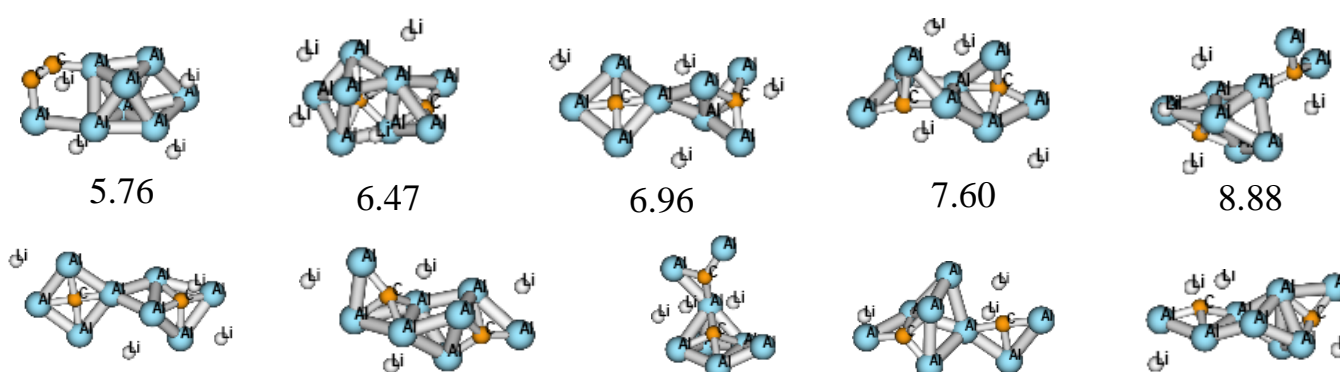

6.47

6.96

7.60

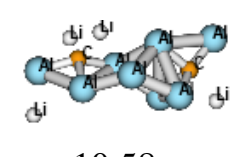

10.10
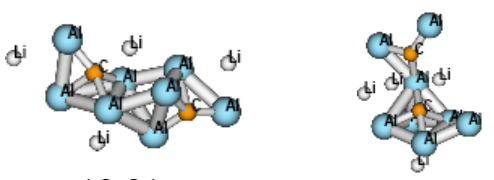

10.47

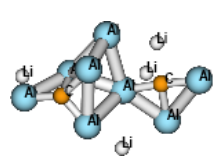

$$
10.31
$$
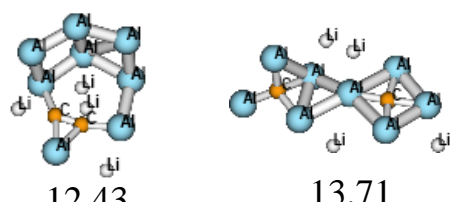

13.71

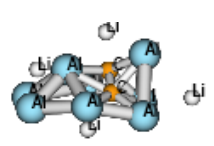

11.71

12.43

14.01 


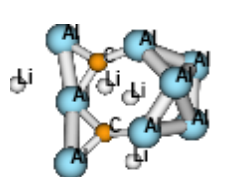

14.94

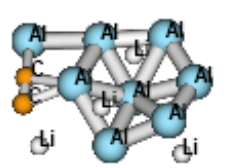

17.31

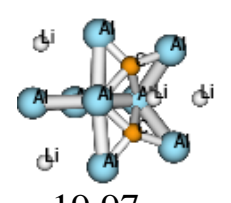

19.97

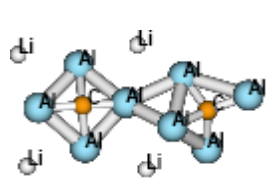

22.91

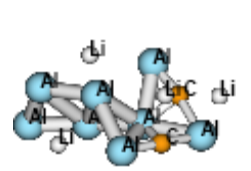

30.72

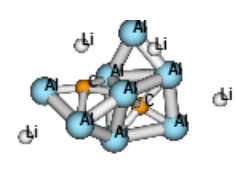

16.42

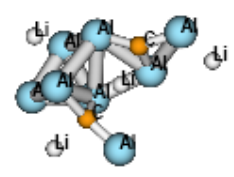

17.89

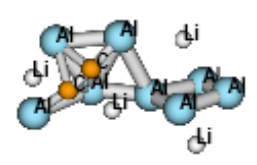

$$
20.81
$$

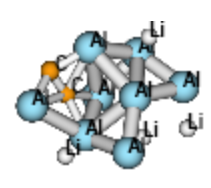

$$
26.20
$$

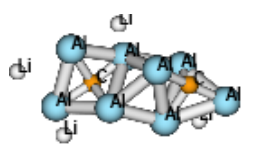

30.79

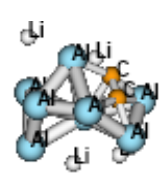

16.69

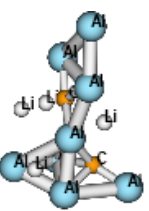

18.16

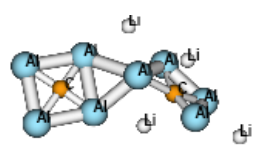

16.94

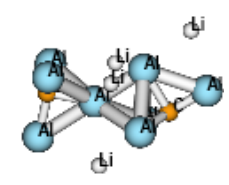

17.09

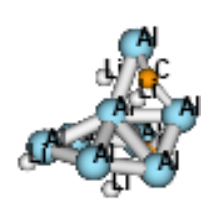

18.36

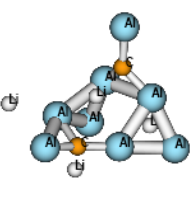

18.69

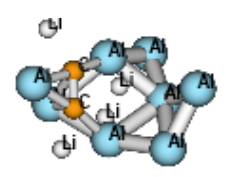

22.51

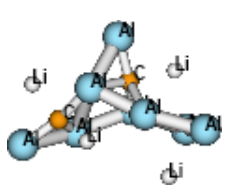

22.58

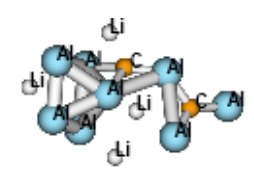

22.62

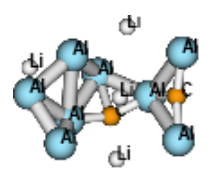

27.39

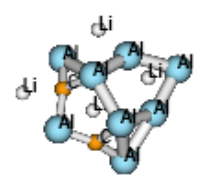

27.98

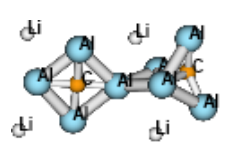

29.30 di di di

34.71 
8. The calculated properties of ${ }^{1} \mathrm{C}_{2} \mathrm{Al}_{8} \mathrm{Na}_{4}$

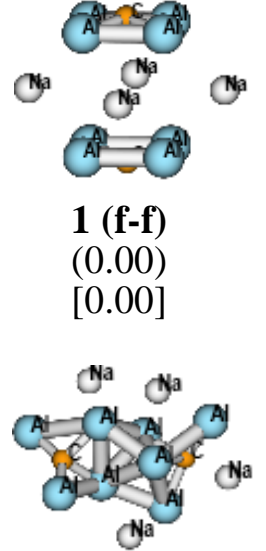

3.30

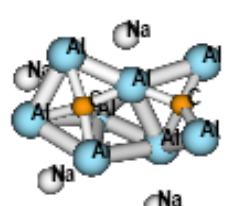

Na

6.81

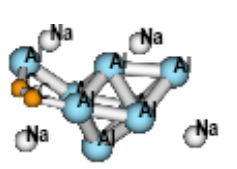

12.24

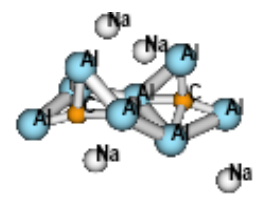

16.75
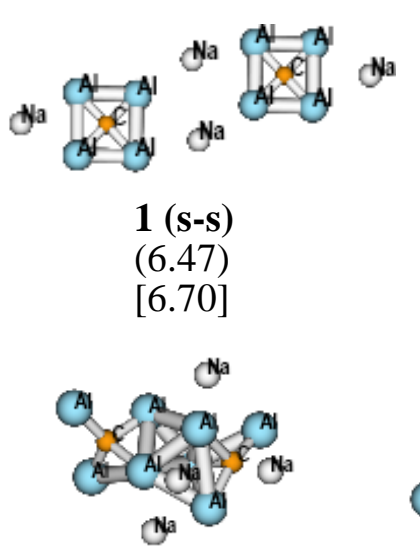

4.21

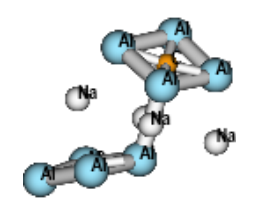

8.69

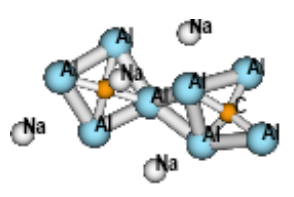

12.05

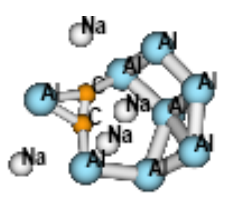

31.66

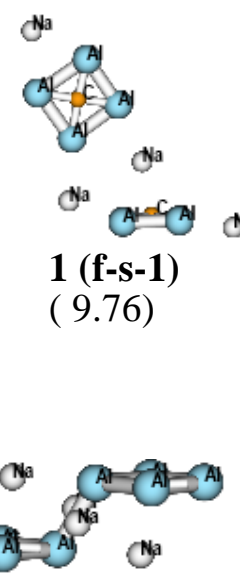

4.29

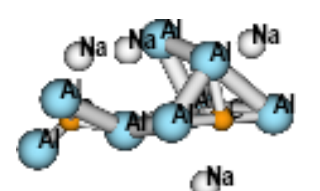

9.47

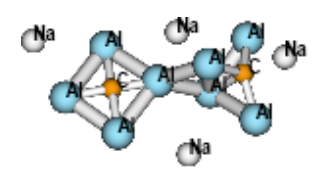

13.94
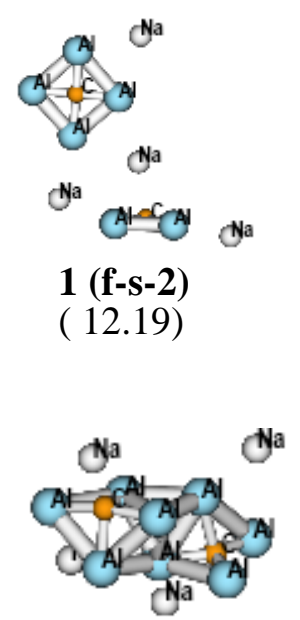

4.37

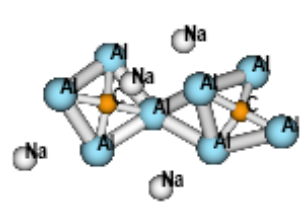

12.02

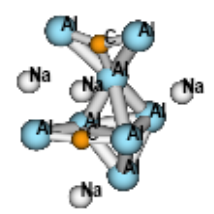

15.77 
9. The calculated properties of ${ }^{1} \mathrm{C}_{2} \mathrm{Al}_{8} \mathrm{~K}_{4}$

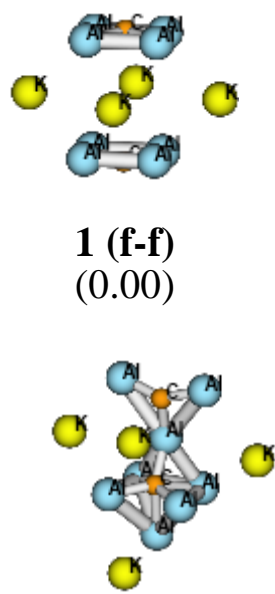

4.41

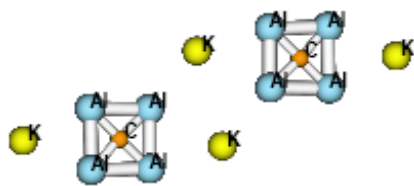

1 (s-s)

(15.10)

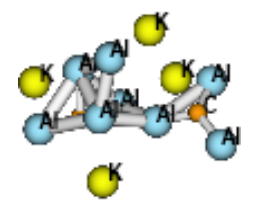

15.64

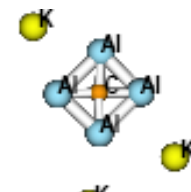

di

1 (f-s-1)

(17.66)

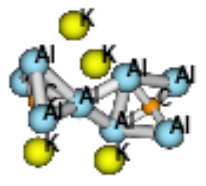

22.98

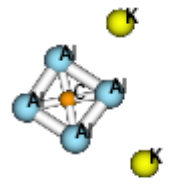

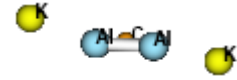

1 (f-s-2)

(19.72)

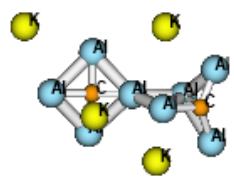

25.93 
10. The calculated properties of ${ }^{1} \mathrm{C}_{2} \mathrm{Al}_{8} B \mathrm{e}_{2}$

af

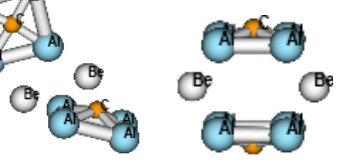

0.00
38.94

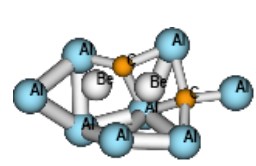

$-13.41$

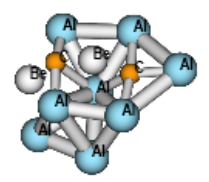

$-24.3$

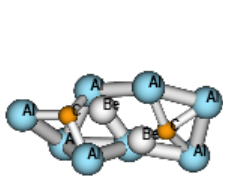

$-22.87$

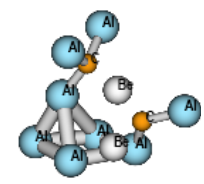

$-20.97$

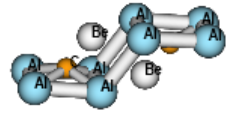

$-15.59$

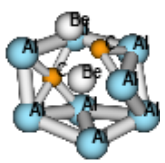

$-13.91$

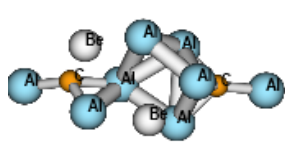

$-10.38$

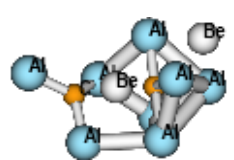

$-9.34$

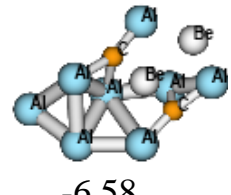

$-6.58$

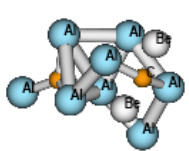

$-3.87$

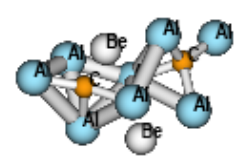

$-1.80$

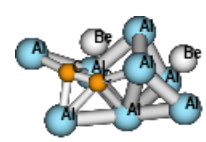

0.29

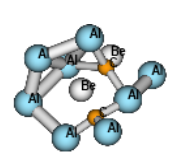

0.85

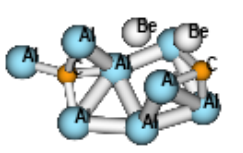

2.51

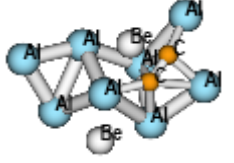

4.61

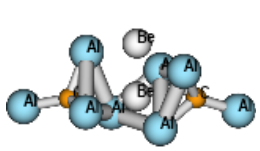

4.87

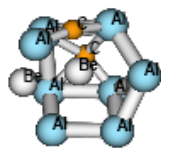

6.27

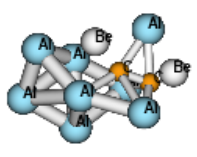

6.40

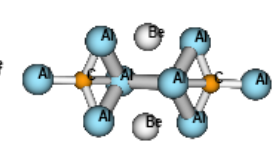

7.67

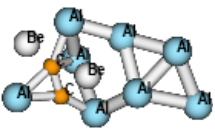

8.95

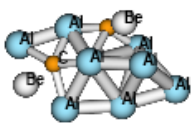

9.32
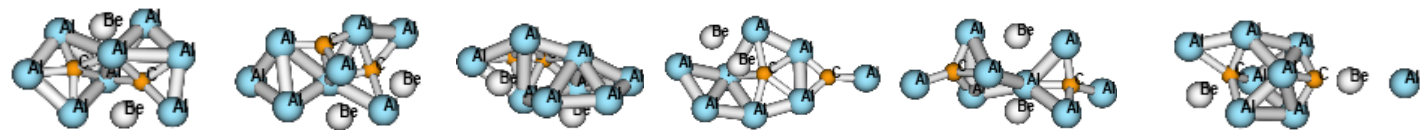

10.26

11.25

11.87

12.84

15.63

17.77

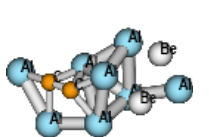

18.05

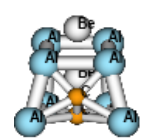

19.62

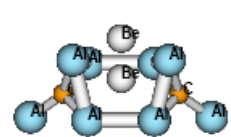

21.29

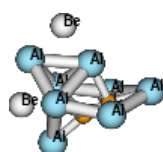

24.30

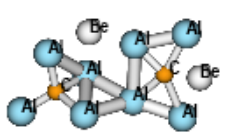

26.14
(A) $B$, $A$ (B) A 27.12

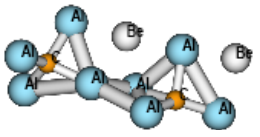

37.25

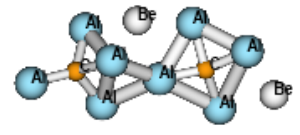

38.20

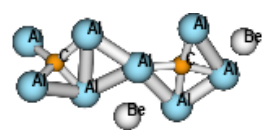

46.45 
11. The calculated properties of ${ }^{1} \mathrm{C}_{2} \mathrm{Al}_{8} \mathrm{Mg}$

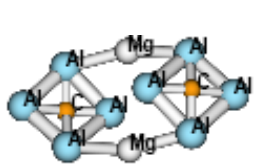

0.00

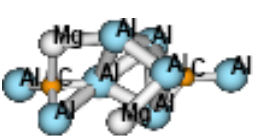

$-9.78$

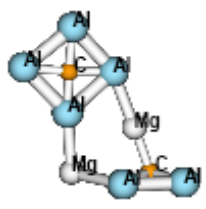

1.98

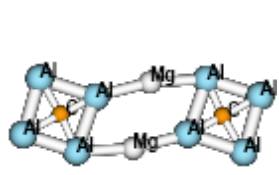

4.13

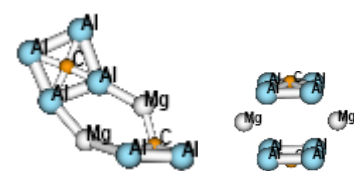

4.71
(4)

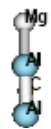

23.77

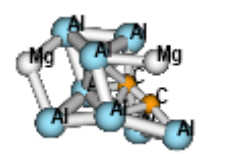

$-0.23$

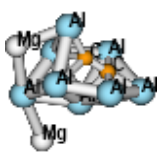

0.58

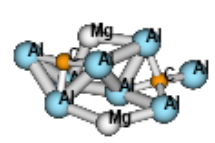

4.57

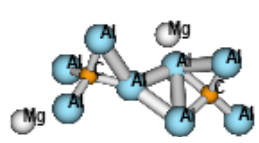

5.65

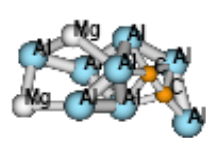

5.72

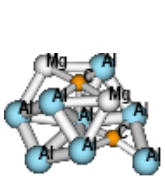

8.45

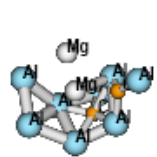

8.52

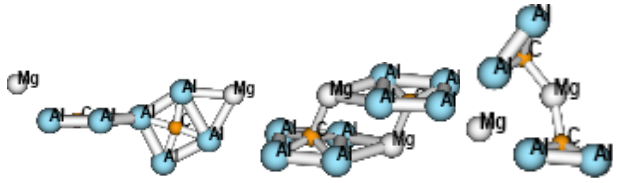

20.60

0.13(i)

9.13(i)

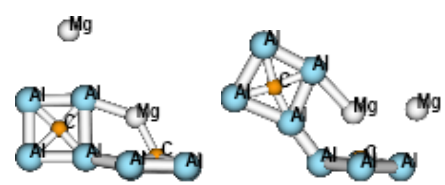

15.85(i) 25.06(i) 
12. The calculated properties of ${ }^{1} \mathrm{C}_{2} \mathrm{Al}_{8} \mathrm{Ca}_{2}$

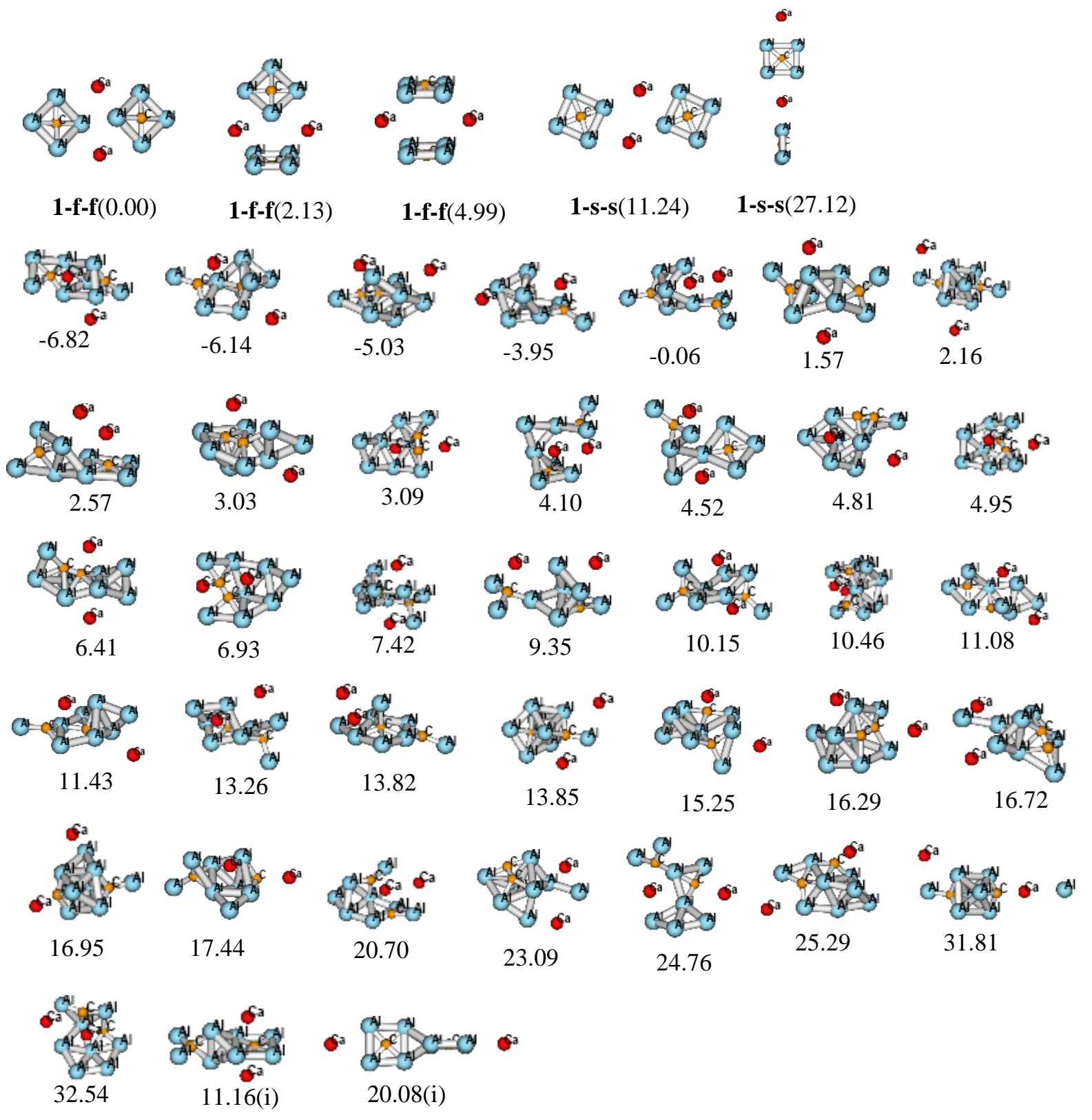




\section{The calculated properties of ${ }^{1} \mathrm{CAl}_{4} \mathrm{LiCp}^{2-}$}

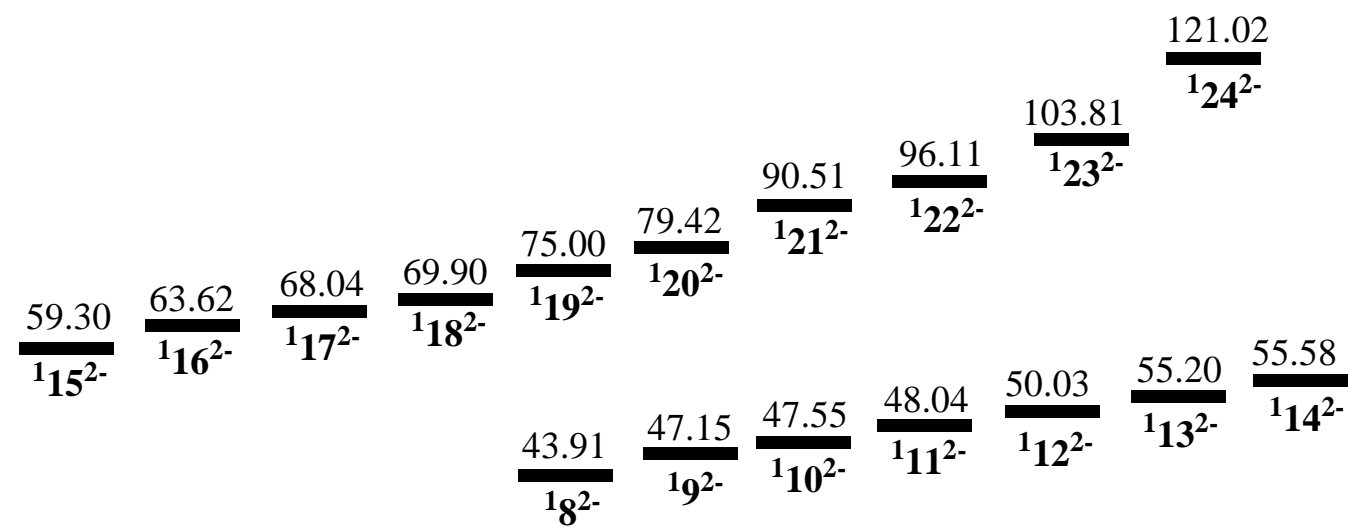

$\frac{20.48}{\mathbf{1}^{\mathbf{2 -}}} \frac{24.03}{\mathbf{1}_{5^{2-}}} \frac{25.32}{\mathbf{1}^{\mathbf{2 -}}} \frac{26.64}{\mathbf{1}^{\mathbf{2}^{2-}}}$

${ }^{\mathbf{1}} \mathbf{1}^{\mathbf{2 -}}$ is sandwich form, $\mathbf{1}^{\mathbf{2 -}}$ is upright form, $\mathrm{CpLiCAl}_{4}{ }^{2-}$

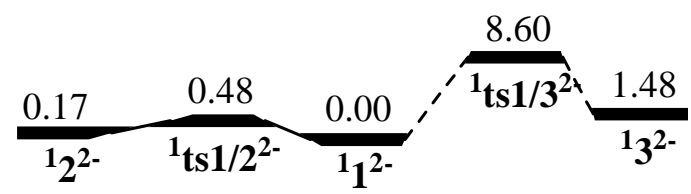

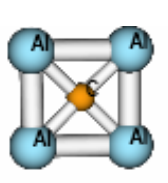

(b)

H.COH

${ }_{1}^{12-}$

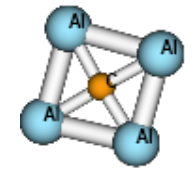

(4)

ate $-\cos ^{2}-\mathrm{H}^{\mathrm{H}}$

${ }^{1}$ ts1/2-

$1^{2-}$

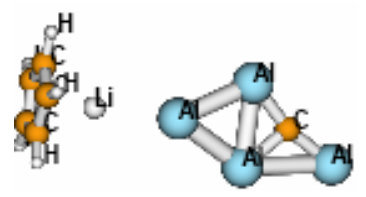

$15^{2-}$

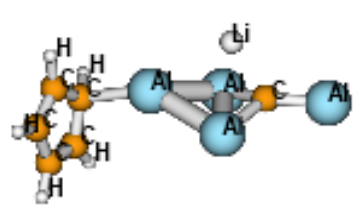

$19^{2-}$
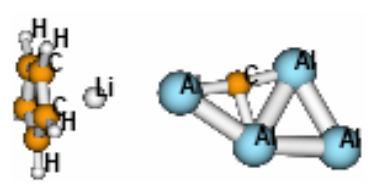

$16^{2-}$

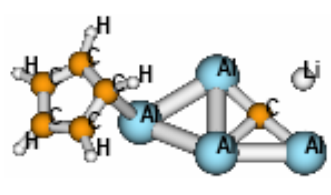

${ }^{1} 10^{2-}$

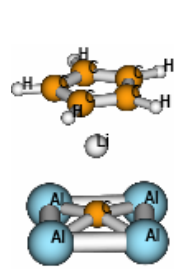

$1^{2-}$
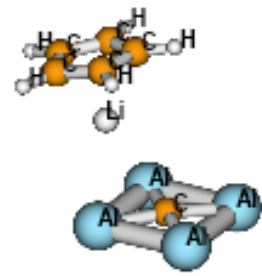

${ }^{1}$ ts1/32-

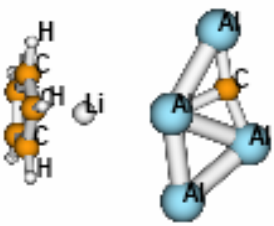

$17^{2-}$

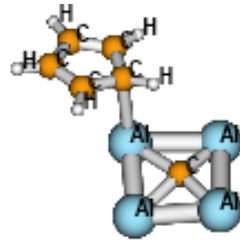

bi

$$
{ }^{1} 1^{2-}
$$
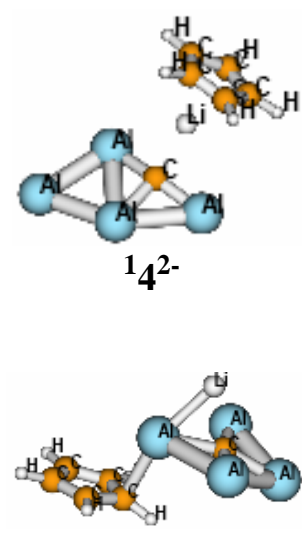

${ }^{1} 8^{2-}$

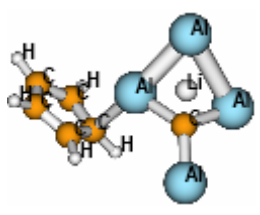

${ }^{1} 12^{2-}$ 

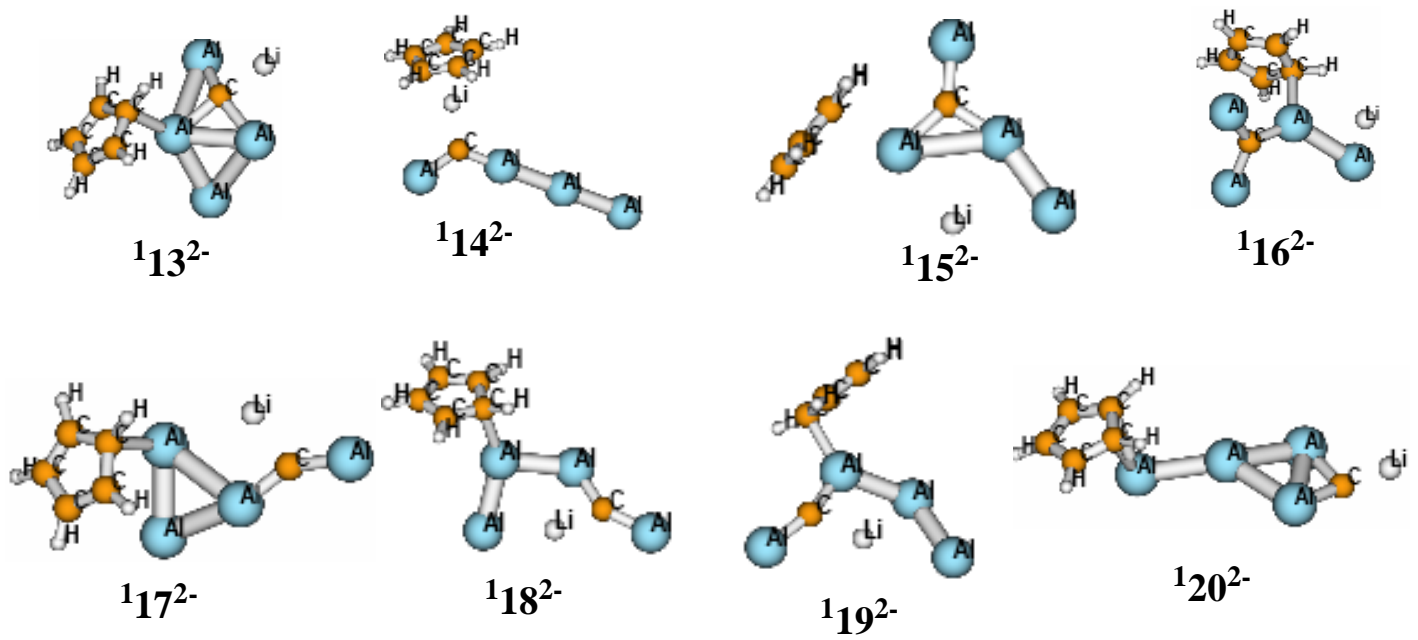

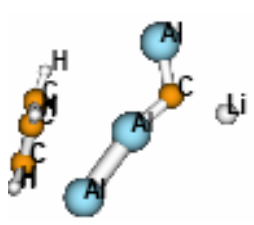

${ }^{1} 21^{2-}$
(A)

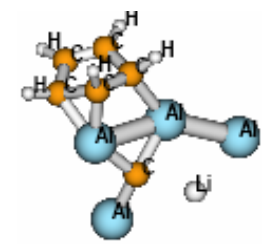

${ }^{1} 22^{2-}$

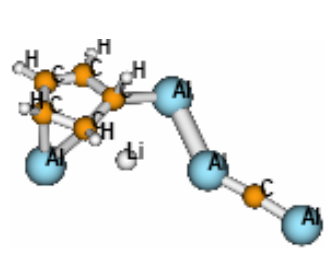

${ }^{1} 23^{2-}$

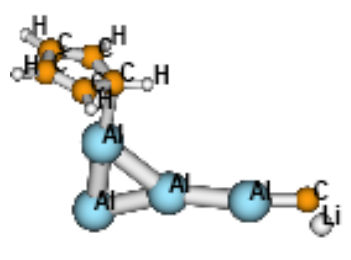

${ }^{1} 24^{2-}$ 
14. The calculated properties of ${ }^{1} \mathrm{CAl}_{4} \mathrm{NaCp}^{2-}$

$$
\begin{aligned}
& \frac{119.77^{\mathbf{1}_{\mathbf{1 3}^{\mathbf{2 -}}}}}{\mathbf{1 4}^{\mathbf{1 3 6 . 9 4}}} \\
& \frac{23.62}{\mathbf{1}^{2-}} \frac{27.98}{\mathbf{1}^{2-}} \frac{28.29}{\mathbf{1}^{2-}} \frac{33.80}{\mathbf{1}^{2-}} \frac{46.15}{\mathbf{1}^{2-}} \\
& \frac{68.39}{\mathbf{1}^{\mathbf{2 -}}} \frac{75.42}{{ }^{\mathbf{1} \mathbf{9}^{\mathbf{2 -}}}} \frac{81.44}{\mathbf{1}^{\mathbf{1 0}}} \frac{91.67}{\mathbf{1}^{\mathbf{1}}} \frac{\frac{93.09}{\mathbf{1 1}^{\mathbf{2 -}}}}{{ }^{\mathbf{1}} \mathbf{1}^{\mathbf{2 -}}} \\
& \frac{0.00}{\mathbf{1}_{\mathbf{1}}^{\mathbf{2 -}}}, \overbrace{\mathbf{1}_{\mathbf{t s} \mathbf{1} / \mathbf{2}^{\mathbf{2}}}}^{8.83}-\frac{5.29}{\mathbf{1}_{\mathbf{2}^{\mathbf{2}}}} \quad \mathbf{1}^{\mathbf{2 -}} \text { is sandwich form, } \mathbf{2}^{\mathbf{2 -}} \text { is upright form, } \mathrm{CpNaCAl}_{4}{ }^{2-} \\
& \text { (A) } \\
& \text { 整 }
\end{aligned}
$$
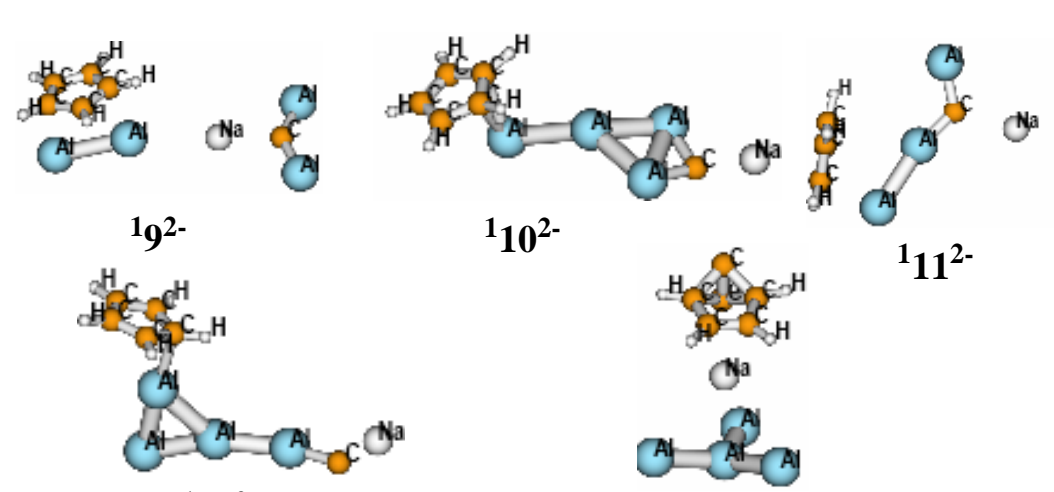
15. The calculated properties of ${ }^{1} \mathrm{CAl}_{4} \mathrm{KCp}^{2-}$

$$
\text { (19.72 }
$$
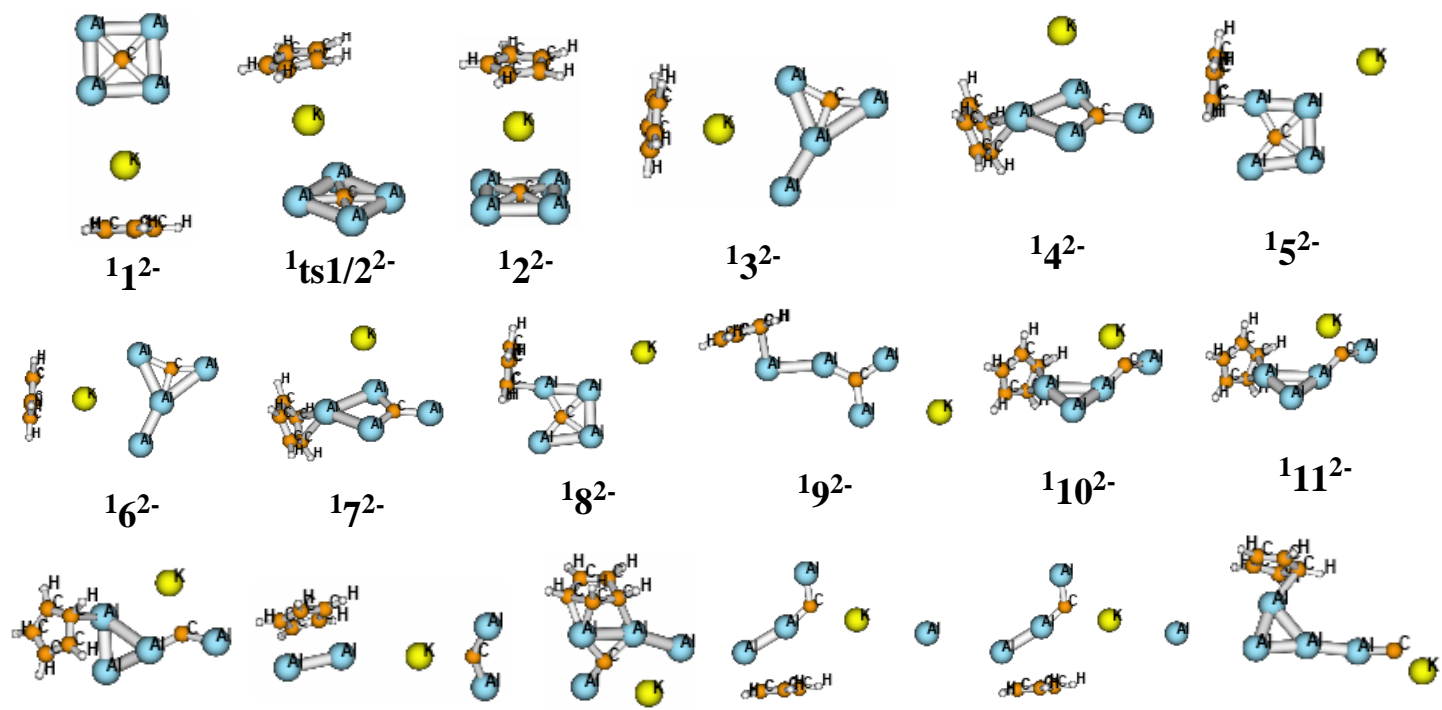
16. The calculated properties of ${ }^{1} \mathrm{CAl}_{4} \mathrm{BeCp}^{-}$

$\frac{113.44}{\mathbf{1}_{\mathbf{2 0}}^{-}}$

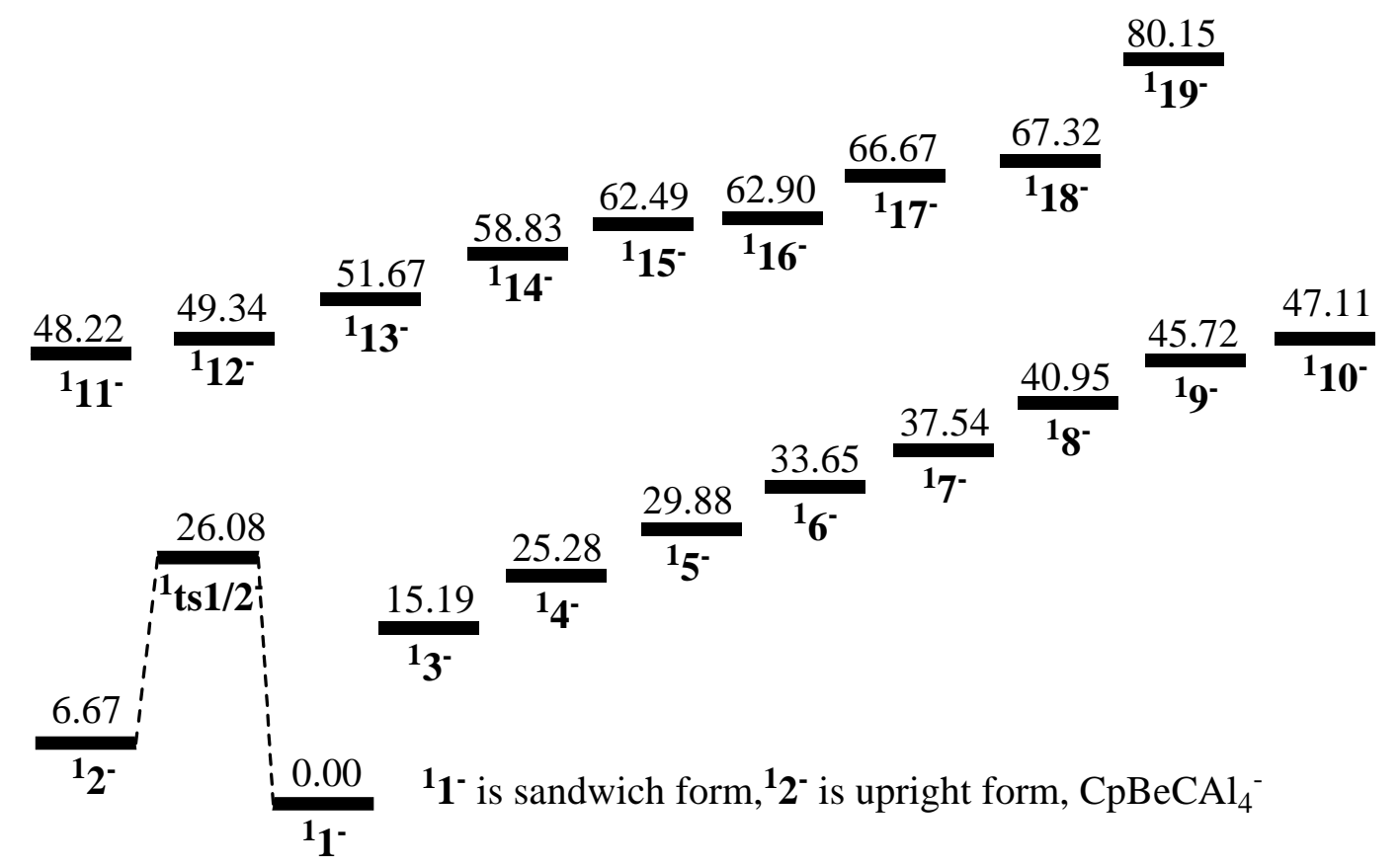

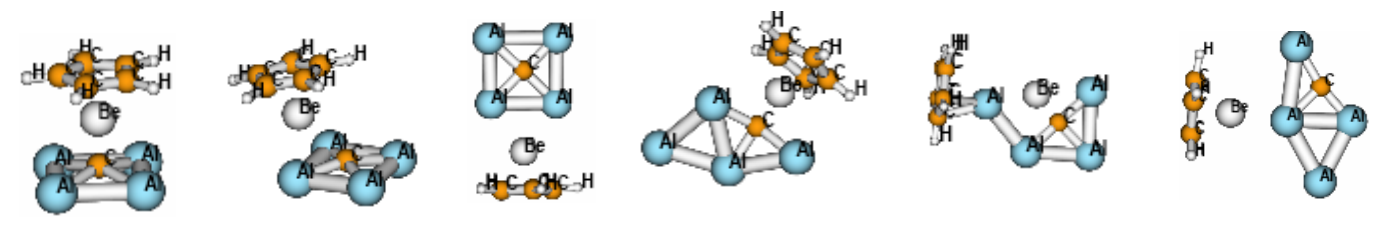

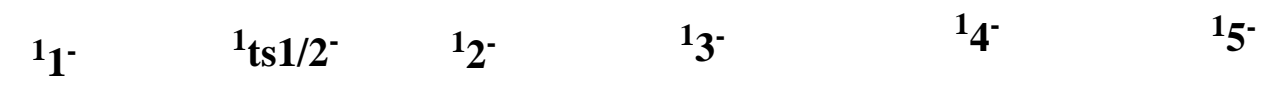

(3)
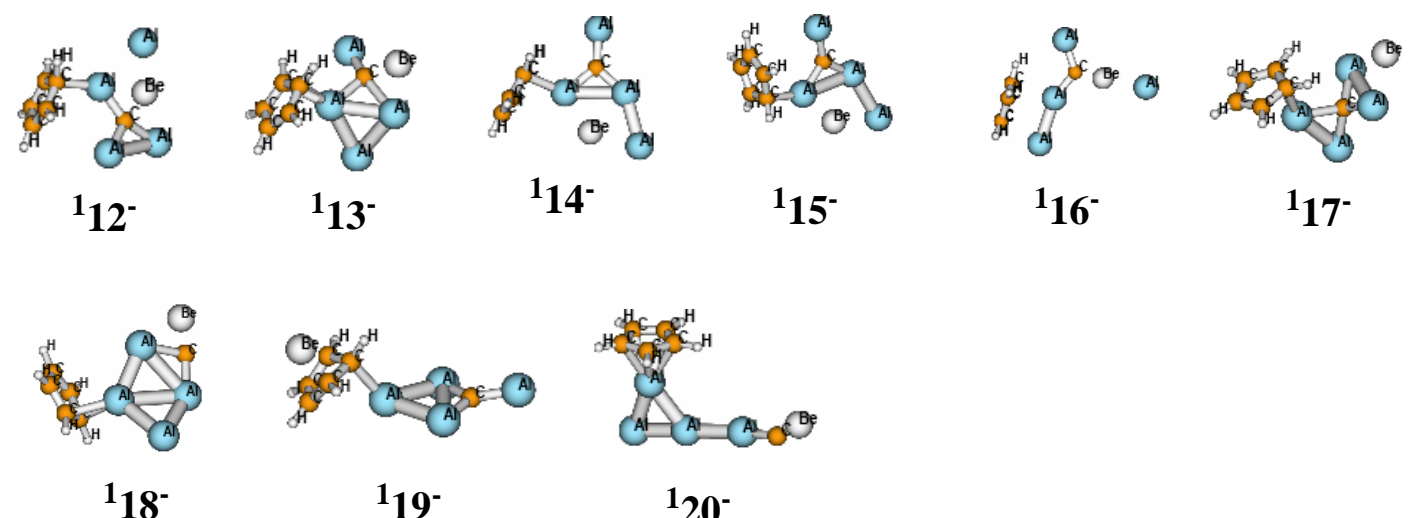
17. The calculated properties of ${ }^{1} \mathrm{CAl}_{4} \mathrm{MgCp}^{-}$

$$
\begin{aligned}
& \frac{112.53}{\mathbf{1}^{-}} \\
& \begin{array}{llll} 
& & \frac{76.54}{\mathbf{1}_{12}^{-}} & \frac{81.11}{\mathbf{1}^{-}}
\end{array} \\
& \frac{50.28}{\mathbf{1}^{-}} \frac{50.93}{\mathbf{1}^{-}} \frac{58.58}{\mathbf{1}^{-}} \\
& \frac{26.09}{\mathbf{1}_{\mathbf{3}^{-}}^{-}} \frac{28.06}{\mathbf{1}_{\mathbf{4}^{-}}} \frac{34.66}{{ }^{\mathbf{1}^{-}}} \frac{39.86}{\mathbf{1}^{-}} \frac{40.04}{\mathbf{1}^{-}} \frac{\frac{41.80}{\mathbf{1}^{-}}}{}
\end{aligned}
$$

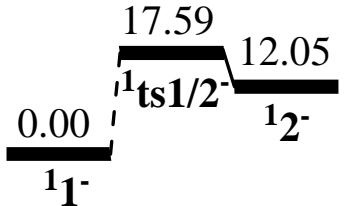

$$
\begin{aligned}
& { }^{1} \mathbf{1}^{-} \text {is sandwich form, }{ }^{\mathbf{1}} \mathbf{2}^{-} \text {is upright form, } \mathrm{CpMgCAl}_{4}^{-} \\
& 1^{-} 1_{151 / 2^{-}} \\
& \text {16- } \mathbf{6}^{-} \\
& \text {(1) } 1 \text { (1) } 12^{-}
\end{aligned}
$$


18. The calculated properties of ${ }^{1} \mathrm{CAl}_{4} \mathrm{CaCp}^{-}$

$$
\begin{aligned}
& \frac{124.13}{\mathbf{1}^{-}} \\
& \frac{93.01}{\mathbf{1}^{-}} \quad \frac{94.45}{\mathbf{1}^{-}} \\
& 45.79{\frac{49.20}{\mathbf{1}^{-}}}^{\frac{56.01}{\mathbf{1}^{-}}}
\end{aligned}
$$

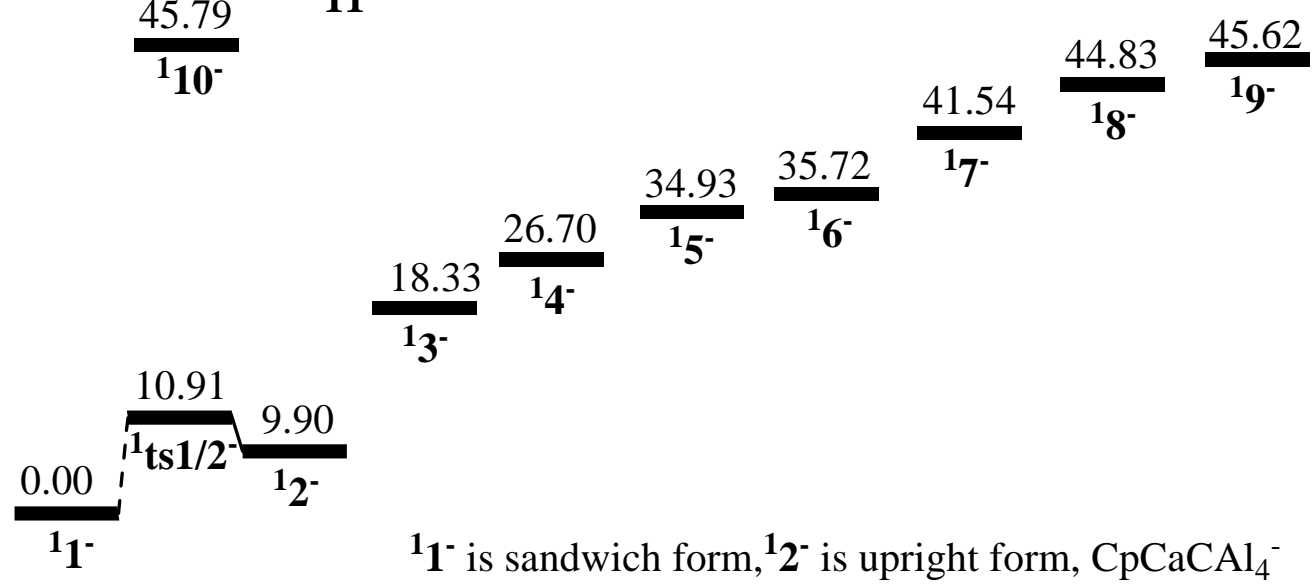
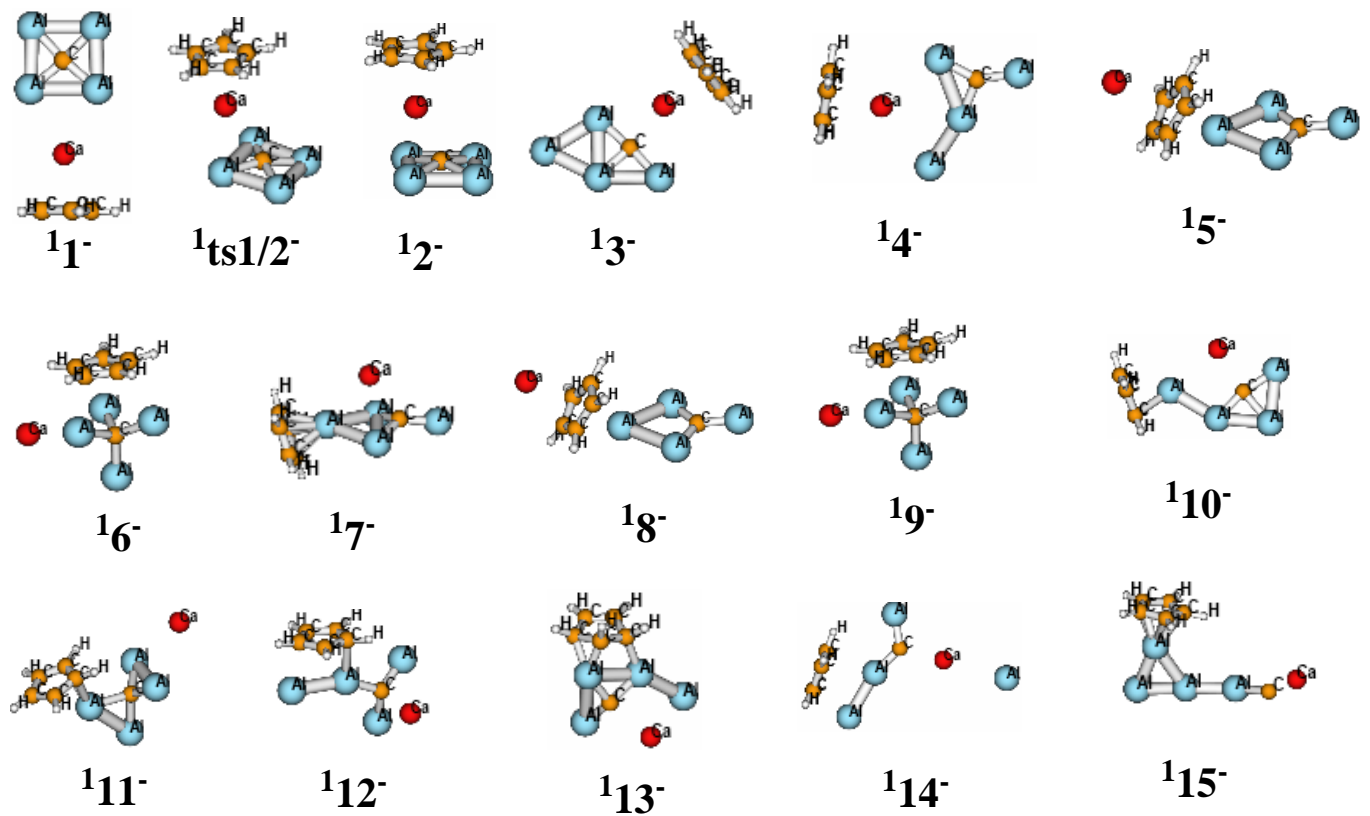
19. The calculated properties of $\left(\mathrm{Li}_{2}\right)^{2+} \mathrm{CAl}_{4} \mathrm{LiCp}^{2-}$

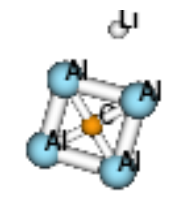

di

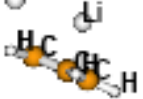

0.00

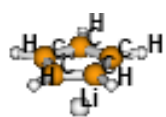

di

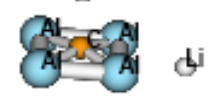

8.16
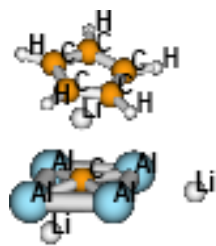

11.70

di

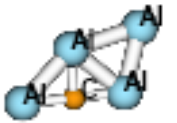

لji

HC $\mathrm{AHC}_{0} \mathrm{H}$

di

29.80 di

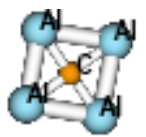

di di

HC. OHC $_{3}$

3.31
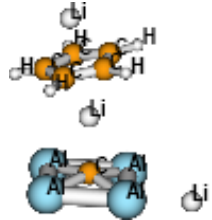

8.41

di

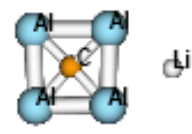

di

HoffeCAH

13.39

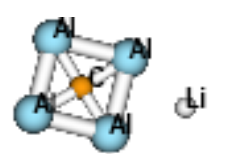

di

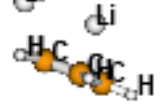

5.42

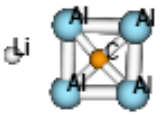

di OHACSCH

di

8.78

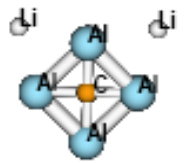

di

HAfIrGH

16.26

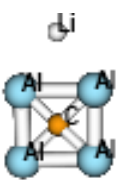

di

o $\mathrm{H}-\mathrm{G}-\mathrm{C}-\mathrm{C}$

di

5.61

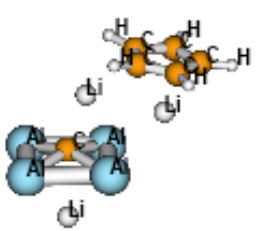

9.01

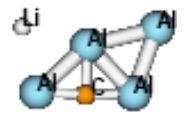

di

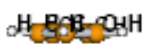

di

23.59 
20. The calculated properties of $\left(\mathrm{Li}_{2}\right)^{2+} \mathrm{CAl}_{4} \mathrm{NaCp}^{2-}$

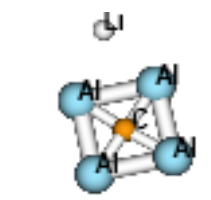

di Na

thof $\mathrm{CO}_{\mathrm{O}} \mathrm{H}$

0.00

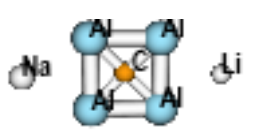

di

H.

8.29

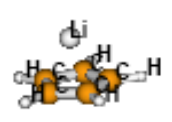

तja

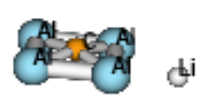

13.55

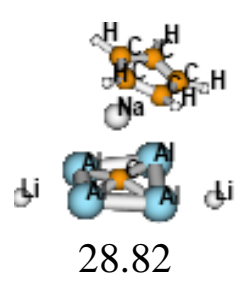

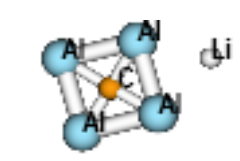

di Na

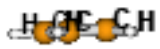

3.47

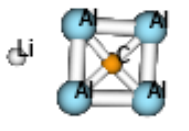

Na

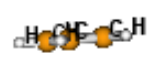

di

9.55
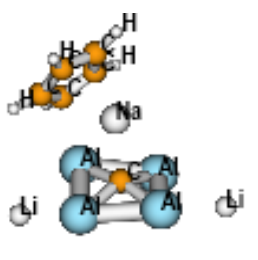

24.53

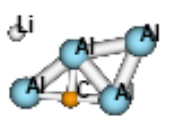

Na

배을 $\mathrm{H}$

di

29.82

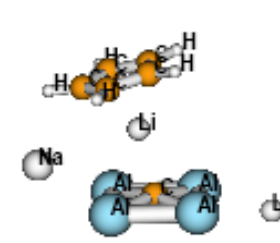

3.87

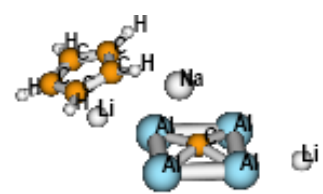

10.45

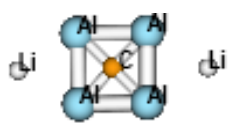

तNa

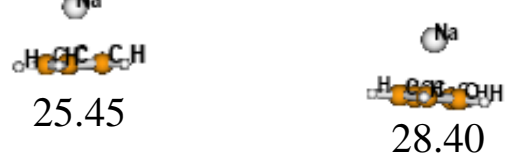

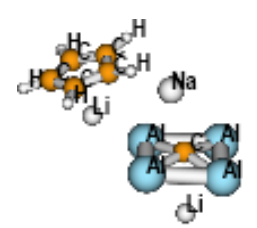

11.39

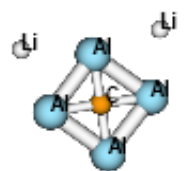

28.40

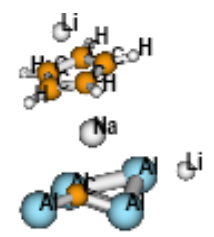

36.45 
21. The calculated properties of $\left(\mathrm{Li}_{2}\right)^{2+} \mathrm{CAl}_{4} \mathrm{KCp}^{2-}$

(i)


22. The calculated properties of $(\mathrm{Li})^{+} \mathrm{CAl}_{4} \mathrm{MCp}^{-}(\mathrm{M}=\mathrm{Be}, \mathrm{Mg}, \mathrm{Ca})$
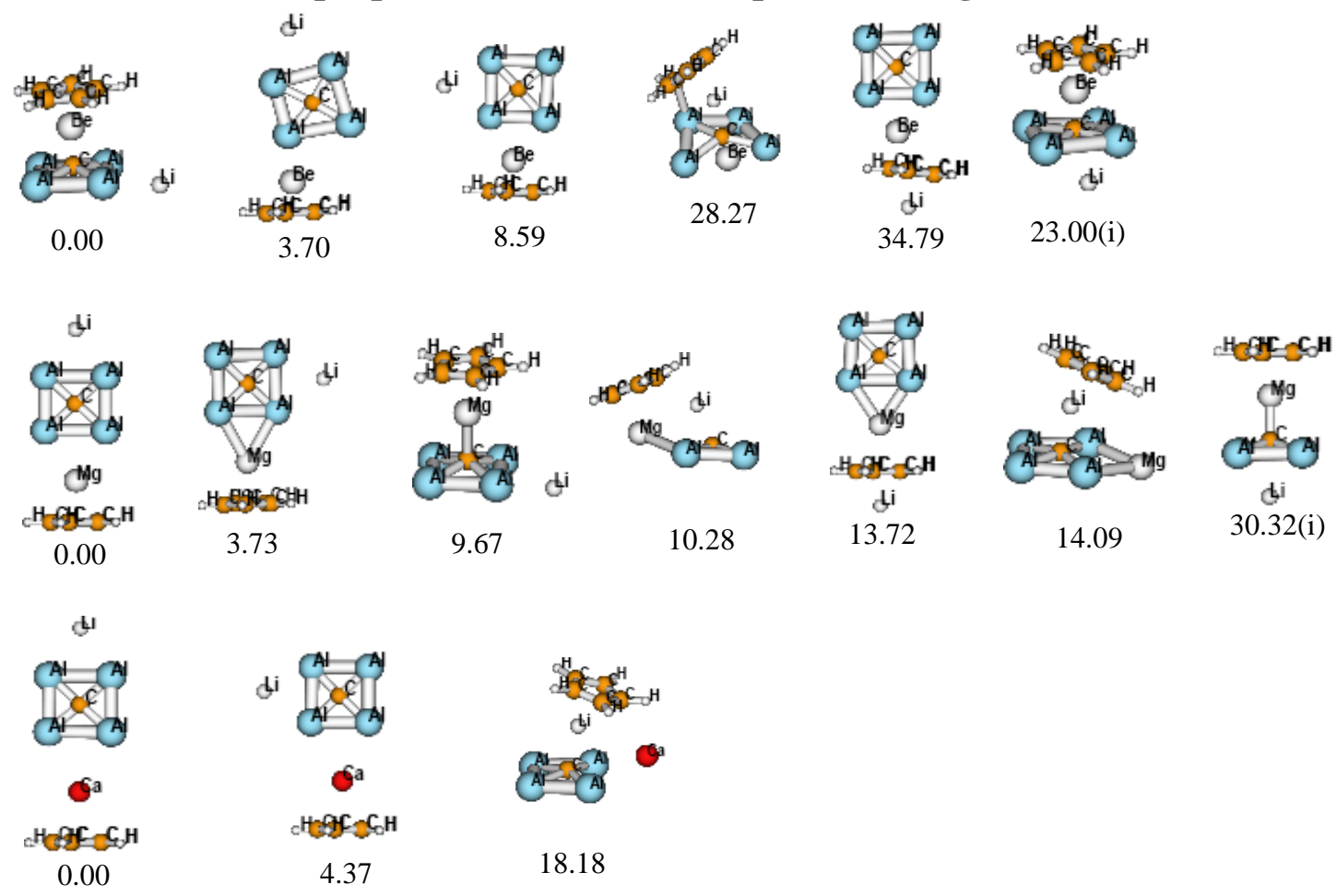
23. The calculated properties of various extend system

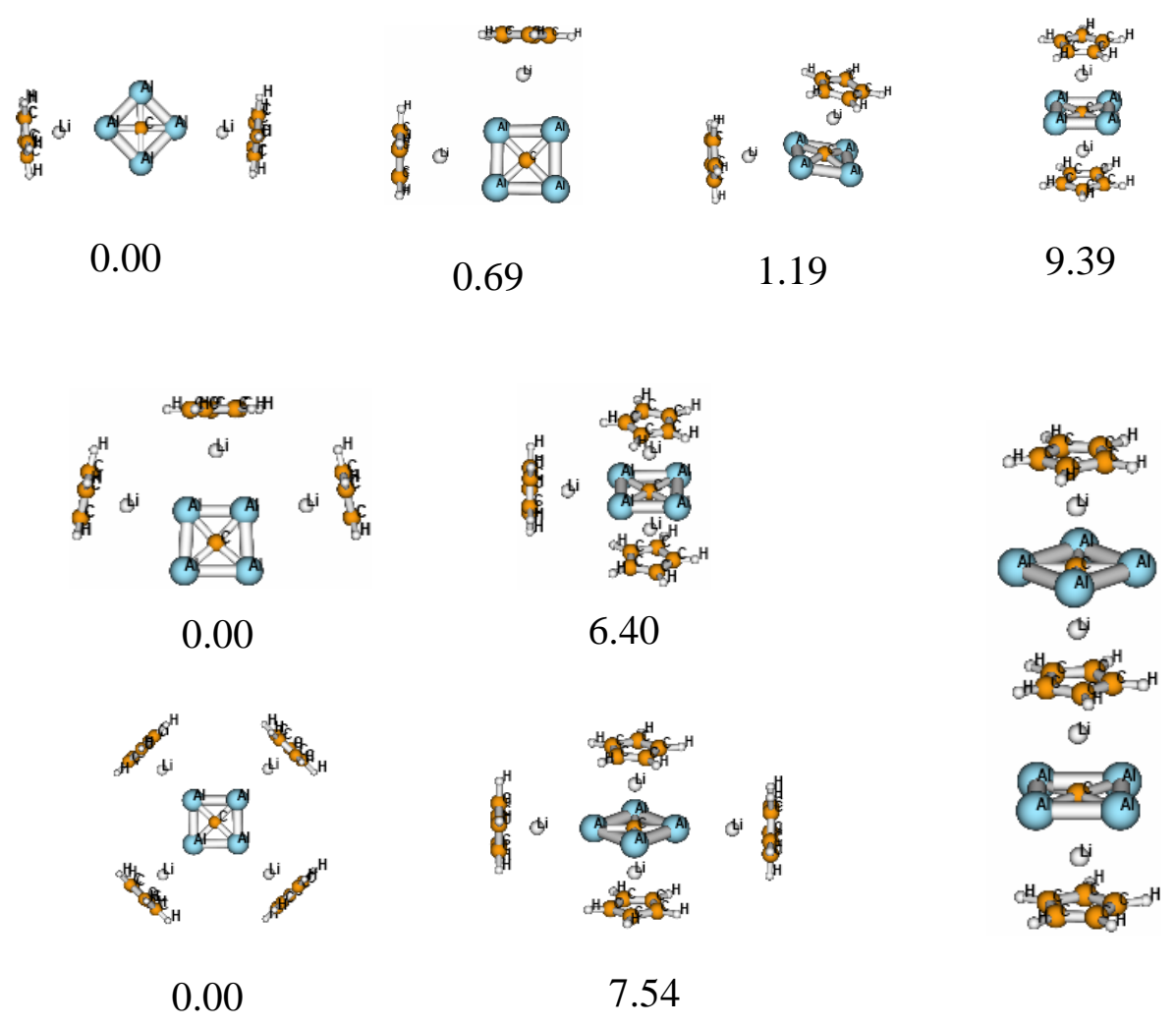

2.65 


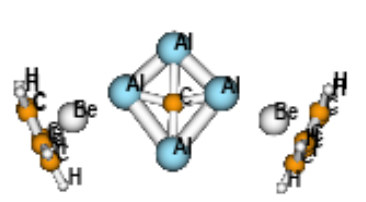

0.00

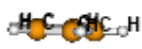

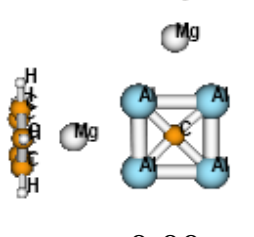

0.00

翼

0.00

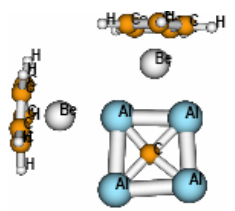

2.46

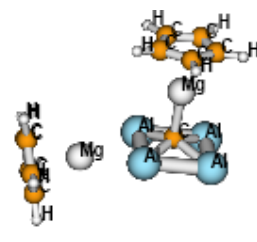

3.85

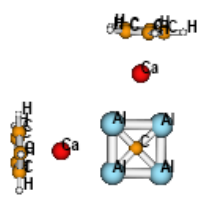

4.72 d.

A

"मet

7.75

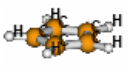

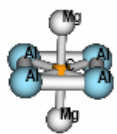

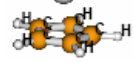

39.31

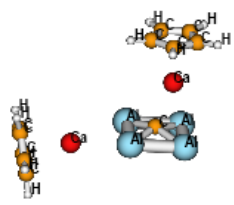

12.48 


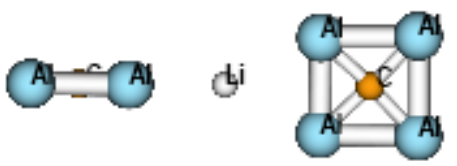

C,0,0.,-2.9378569991,2.9378569991

Al,0,-1.0048230926,-3.1980122783,4.6190259078 Al,0,-0.9712313018,-1.2445982858,2.618106645 Al,0,0.9712313018,-2.618106645,1.2445982858 Al,0,1.0048230926,-4.6190259078,3.1980122783 $\mathrm{Li}, 0,0 ., 0 ., 0$.

Al,0,0.9712170748,2.618116705,-1.2445882258 Al,0,-0.9712170748,1.2445882258,-2.618116705 Al,0,1.0048083735,4.6190363157,-3.1980018704 Al,0,-1.0048083735,3.1980018704,-4.6190363157 C,0,0.,2.9378569991,-2.9378569991

\section{HF $=-2023.1472094$}

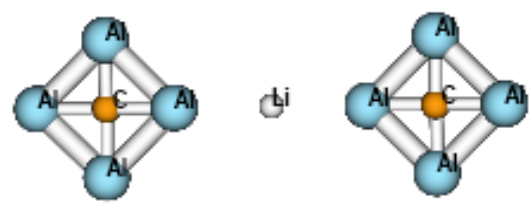

Li,0,0.,0.,0.

Al,0,1.406467036,4.3493772946,-2.360303392 Al,0,-0.0000001838,1.9663986277,-1.9663988875 Al,0,-0.0000004437,4.746805515,-4.7468061422 $\mathrm{Al}, 0,-1.4064676631,2.3603026858,-4.3493774751$ C,0,-0.0000003125,3.3436914543,-3.3436918962 Al,0,0.0000001838,-1.9663986277,1.9663988875 Al,0,0.0000004437,-4.746805515,4.7468061422 C,0,0.0000003125,-3.3436914543,3.3436918962 Al,0,1.4064676631,-2.3603026858,4.3493774751 Al,0,-1.406467036,-4.3493772946,2.360303392

\section{$\mathrm{HF}=-\mathbf{2 0 2 3 . 1 4 7 1 5 7 1}$}

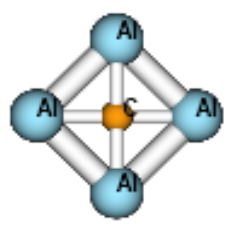

ci

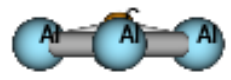

C,0,-3.2732793067,0.,0.

$\mathrm{Al}, 0,-3.7051651358,0 ., 1.973065181$

Al,0,-3.6993025423,1.9720991684,0.

$\mathrm{Al}, 0,-3.7051651358,0 .,-1.973065181$ 
Al,0,-3.6993025423,-1.9720991684,0.

Li,0,-1.0753563171,0.,0.

Al,0,1.7448002643,0.,0.

$\mathrm{Al}, 0,5.6834758664,0 ., 0$.

C,0,3.6997010102,0.,0.

$\mathrm{Al}, 0,3.7160041793,0 ., 1.9888387286$

$\mathrm{Al}, 0,3.7160041793,0 .,-1.9888387286$

\section{HF $=-\mathbf{2 0 2 3 . 1 3 3 9 8 3 1}$}

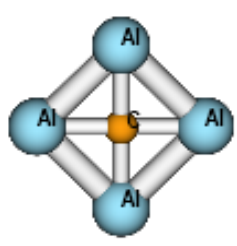

(t)

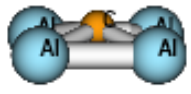

C,0,-3.2724655452,0.,0.

Al,0,-3.70261036,0.7517014381,1.8236595185

Al,0,-3.70261036,1.8210551934,-0.757988835

Al,0,-3.70261036,-0.7517014381,-1.8236595185

Al,0,-3.70261036,-1.8210551934,0.757988835

Li,0,-1.0746611341,0.,0.

Al,0,1.7449739382,0.,0.

$\mathrm{Al}, 0,5.6838109303,0 ., 0$.

C,0,3.6999949028,0.,0.

Al,0,3.7161670263,-0.7611575067,1.837596786 Al,0,3.7161670263,0.7611575067,-1.837596786

HF $=-2023.1339961$

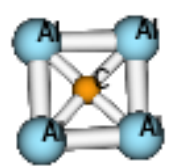

bi

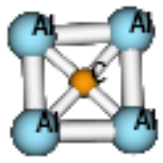

C,0,0.,-2.983694456,2.983694456

Al,0,0.,-2.9465900743,4.9594224258

Al,0,0.,-1.0050428218,2.9507133586

Al,0,0.,-2.9507133586,1.0050428218

$\mathrm{Al}, 0,0 .,-4.9594224258,2.9465900743$

$\mathrm{Li}, 0,0 ., 0 ., 0$.

Al,0,0.,2.9507133586,-1.0050428218

Al,0,0.,1.0050428218,-2.9507133586

Al,0,0.,4.9594224258,-2.9465900743

$\mathrm{Al}, 0,0 ., 2.9465900743,-4.9594224258$

C,0,0.,2.983694456,-2.983694456 


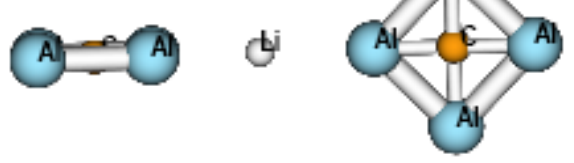

C,0,0.0000002939,-3.139135844,3.1391362598

$\mathrm{Al}, 0,-1.004445756,-3.4013982727,4.8218793573$

Al,0,-0.9728999888,-1.4499900545,2.8258588181 Al,0,0.9729003892,-2.825858535,1.4499903377 Al,0,1.0044465261,-4.8218788127,3.4013988174 Li,0,0.0000000234,-0.2498852522,0.2498852853 Al,0,1.4053686275,4.1352323804,-2.1477118832 Al,0,-0.0000001635,1.7463728234,-1.7463730548 Al,0,-0.0000004244,4.5321534292,-4.5321540296 Al,0,-1.4053692159,2.1477112038,-4.1352325333 C,0,-0.000000293,3.1289994523,-3.1289998667 HF $=-\mathbf{2 0 2 3 . 1 4 8 6 3 3 7}$
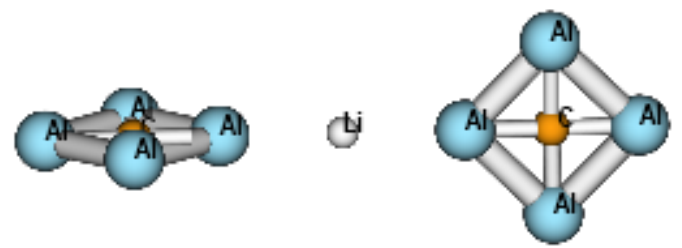

Li,0,0.,0.,0.

$\mathrm{Al}, 0,1.4063157702,4.3413913958,-2.3525314147$ Al,0,-0.0000001839,1.9583645237,-1.9583647822 Al,0,-0.0000004449,4.7387371994,-4.7387378251 Al,0,-1.4063163987,2.3525307095,-4.3413915744 C,0,-0.0000003132,3.3357268068,-3.3357272473 $\mathrm{Al}, 0,0.0000001839,-1.9583645237,1.9583647822$ Al,0,0.0000004449,-4.7387371994,4.7387378251 C,0,0.0000003132,-3.3357268068,3.3357272473 Al,0,-1.4063363778,-2.3525454132,4.3413771347 $\mathrm{Al}, 0,1.4063370062,-4.3413766921,2.3525458544$ HF $=-2023.148405$

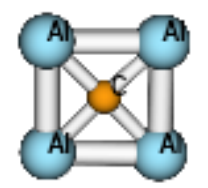

bi

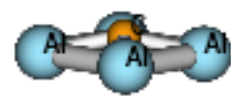

C,0,-2.976191555,0.,0. 
Al,0,-3.4009420933,0.,1.9735760299

Al,0,-3.3974799442,1.9700641228,0.

Al,0,-3.4009420933,0.,-1.9735760299

Al,0,-3.3974799442,-1.9700641228,0.

Li,0,-0.7338702098,0.,0.

Al,0,1.9935601262,0.,-1.3731474158

$\mathrm{Al}, 0,4.7876806122,0 ., 1.4234822722$

C,0,3.4175789561,0.,0.

Al,0,1.9935601262,0.,1.3731474158

$\mathrm{Al}, 0,4.7876806122,0 .,-1.4234822722$

\section{HF $=-2023.1317489$}
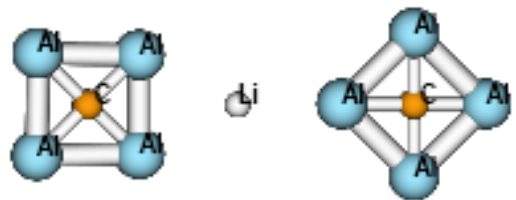

C,0,0.,-3.1570321727,3.1570321974^M Al,0,0.,-3.1230449931,5.1340371101^M $\mathrm{Al}, 0,0 .,-1.1822805651,3.1291063092 \wedge \mathrm{M}$ Al,0,0.,-3.1291062999,1.1822805896^M $\mathrm{Al}, 0,0 .,-5.1340370856,3.123045033 \wedge^{\wedge} \mathrm{M}$ $\mathrm{Li}, 0,0 .,-0.2528247419,0.2528247439 \wedge \mathrm{M}$ $\mathrm{C}, 0,0 ., 3.1460668674,-3.146066892^{\wedge} \mathrm{M}$ $\mathrm{Al}, 0,0 ., 1.7636838618,-1.7636838756 \wedge \mathrm{M}$ $\mathrm{Al}, 0,0 ., 4.5496828979,-4.5496829335^{\wedge} \mathrm{M}$ $\mathrm{Al}, 0,0 ., 4.5650383471,-1.7534689541 \wedge \mathrm{M}$ $\mathrm{Al}, 0,0 ., 1.7534689183,-4.5650383609 \wedge \mathrm{M}$

\section{$H F=-2023.1464761$}

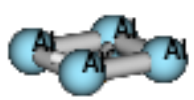

bi

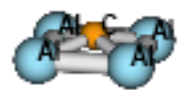

Al,0,1.8265240966,-0.7339084055,-2.7049437942 Al,0,-0.7339084055,-1.8265240966,-2.7049437942 Al,0,-1.8265240966,0.7339084055,-2.7049437942 Al,0,0.7339084055,1.8265240966,-2.7049437942 Al,0,-0.7339399782,1.8265114101,2.7049437942 Al,0,-1.8265114101,-0.7339399782,2.7049437942 Al,0,0.7339399782,-1.8265114101,2.7049437942 Al,0,1.8265114101,0.7339399782,2.7049437942 C,0,0.,0.,2.2553155537 C,0,0.,0.,-2.2553155537 $\mathrm{Li}, 0,0 ., 0 ., 0$. 
Al,0,1.3810774866,1.4006182872,2.7112870925 Al,0,-1.4002236629,1.3819594037,2.7116275899 Al,0,-1.3822023044,-1.3999656175,2.711047536 Al,0,1.4003658334,-1.3813141734,2.710704102 Al,0,1.4006058208,1.3811468628,-2.7112763664 Al,0,-1.3999470603,-1.3822224901,-2.7110556021 Al,0,1.3820245133,-1.400198549,-2.7116560825 Al,0,-1.3813386518,1.4003587002,-2.7106757991 C, $0,-0.0002986403,-0.000255836,2.2516568756$ C,0,-0.0002266397,-0.0003042058,-2.2516665293 Li,0,-0.0005179972,-0.0005370865,0.0000086028

\section{$H F=-2023.1134869$}

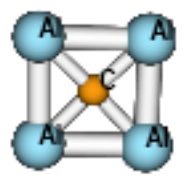

bi

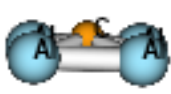

C,0,-2.9776654736,0.,0.

Al,0,-3.4022567197,0.750317923,1.8232308579 Al,0,-3.4022567197,1.8197737968,-0.7586640069 Al,0,-3.4022567197,-0.750317923,-1.8232308579 Al,0,-3.4022567197,-1.8197737968,0.7586640069 Li,0,-0.7341800049,0.,0.

Al,0,1.996487439,0.5254108095,-1.2684538971 Al,0,4.7905298807,-0.5447769774,1.3152079621 C,0,3.4205719947,0.,0. Al,0,1.996487439,-0.5254108095,1.2684538971 Al,0,4.7905298807,0.5447769774,-1.3152079621 HF $=-2023.1316499$ 
৫্

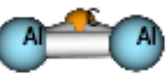

Al,0,2.2249144612,-0.5081397692,-1.878135595 Al,0,3.4792775621,-1.8993026779,0.1823465347 Al,0,2.2877906519,1.8477437392,-0.296464552 Al,0,3.5412310979,0.421212081,1.7402667275 Al,0,-2.6800286662,0.4101710126,1.9926832939 Al,0,-3.0433879881,1.9565579514,-0.2956336871 Al,0,-3.1052538245,-0.3513048435,-1.8474587415 Al,0,-2.7419896182,-1.9004913049,0.4389740179 C,0,2.6468622439,-0.085105887,0.0216026317 C,0,-2.4378766406,0.0522348656,0.0190607977 Li,0,-0.2557038032,0.1678085586,-0.2398315188 HF $=-2023.1081183$

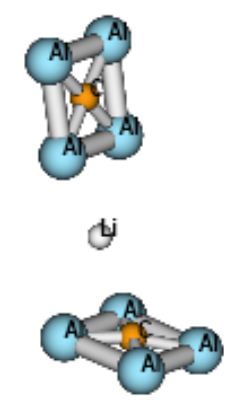

C,0,-3.4338841567,0.1435190431,-0.1126511063 Al,0,-3.0836103717,-0.3666242916,1.7790143846 Al,0,-4.2058107896,1.8652475421,0.5448840157 Al,0,-4.1803624483,0.5360031885,-1.9240900934 Al,0,-3.0578183535,-1.7133660225,-0.7224582775 Li,0,-0.4311771223,-0.356846984,0.1875425612 Al,0,2.2503065468,-0.7843085444,-1.1177873745 Al,0,5.0117705588,0.6692766186,1.3073673631 C,0,3.6551146212,-0.0672404003,0.0738694291 Al,0,2.2252647563,0.520509263,1.3058599691 Al,0,5.0376561457,-0.6795939772,-1.1981698041 HF =-2023.1246908

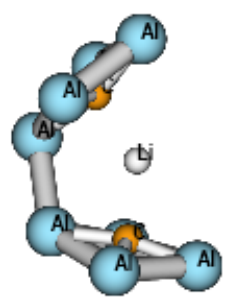


$\mathrm{Al}, 0,-2.2731206552,2.7356668846,0.5940396813$ Al,0,-3.3105641861,0.629972845,-0.8955936731 Al,0,-1.1229213883,0.6615005084,2.3279972327 Al,0,-1.8174299363,-1.2480051774,0.3573611428 Al,0,0.9700298042,-1.2360459518,-2.1569138057 Al,0,0.9694556603,-1.9237967908,0.5887436241 Al,0,3.3157676685,-0.6085689812,0.8895680369 Al,0,3.1719476536,0.6269104865,-1.5968798283 C,0,-1.7867812974,0.8190055403,0.4408158933 C,0,1.8835147174,-0.457117155,-0.5489245102 Li,0,0.2261531372,0.8464766623,-0.253179879 HF $=-2023.1025973$

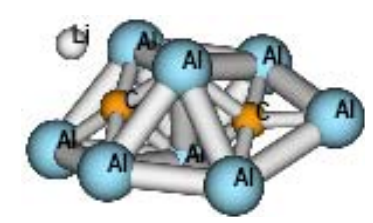

Al,0,-1.3767585385,-2.0898245281,-0.2295562343 Al,0,0.2092557838,-0.0030566202,-1.3026252619 Al,0,-1.5123301661,1.9900297643,-0.2705966017 Al,0,-3.5005028958,-0.1178113022,-0.1113407043 Al,0,3.1199606084,0.1046820772,-0.8248785269 Al,0,-0.2279749122,0.0093998073,1.5059894666 Al,0,1.4665192073,-2.0203522464,0.299208407 Al,0,1.3290522917,2.1142170762,0.2750555206 C,0,1.4702487285,0.0493025787,0.3792194804 C,0,-1.5069585621,-0.0513542466,-0.1042946121 Li,0,2.2087936929,0.0592058808,2.3047073147 HF $=-2023.1161064$

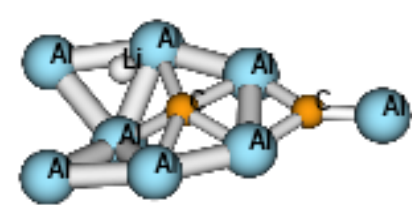

Al,0,-2.2531415882,2.932198994,-1.0118462517 Al,0,-3.78734744744,-0.5735652842,-0.2823863417 Al,0,-1.6029362983,0.824253485,0.6259644048 Al,0,-1.3887699415,-1.7336106484,0.0239902114 Al,0,0.2694072762,2.0588719243,-0.7596050004 Al,0,4.5610817463,-1.7086165317,1.1216592964 Al,0,2.051761076,0.5236552389,0.049665459 C,0,0.0414277582,-0.0697587979,-0.2163743838 Li,0,-1.0492514482,0.0338540364,-2.0259678497 Al,0,1.0483574695,-1.7734148672,0.5232234692 C,0,2.8699713318,-1.1383415604,0.5995836066 


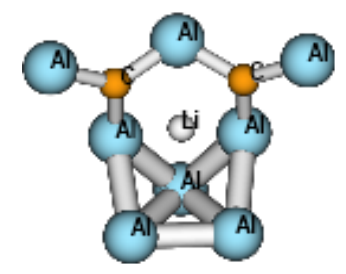

Al,0,-3.1359256246,-2.231446105,0.796525367 Al,0,-0.6646597961,3.7951491251,0.7986323075 Al,0,1.8456498403,-2.2051305054,0.8065109618 Al,0,2.8641186062,0.2623690449,0.8032775217 Al,0,1.9127063582,-0.7858135742,-1.6374584107 Al,0,-0.3164848272,-1.6284424697,-0.7641378085 Al,0,-2.1370702311,0.8787899522,-0.4728370761 Al,0,0.919832985,1.3826860271,-0.7616338779 Li,0,-0.4660141562,0.1933902357,1.3397913889 C,0,-0.6642628524,2.0268484225,0.1314216159 C,0,-1.8937592429,-0.9712267796,0.1327782223

\section{HF $=-2023.0811146$}

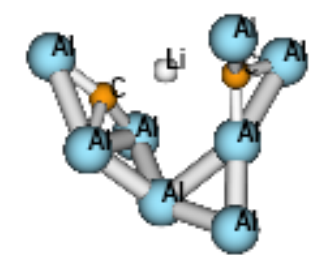

Al,0,0.0369732535,1.2119540513,3.4682818052 Al,0,-0.3812190084,1.6128374647,0.9815135557 Al,0,0.035673187,-2.6493537079,0.4997847707 Al,0,1.0284266658,-0.7090740746,2.0201257555 Al,0,2.2515834873,-1.0774573969,-0.4980110334 Al,0,0.1106117295,-3.3135909211,-2.4467192713 Al,0,-2.8847618578,1.7427568349,-1.2950506104 Al,0,0.0510444329,3.2834252467,-1.4237954684 Li,0,-0.3515100992,-0.1232687079,-1.4715249654 C,0,0.6743781978,-1.9535888479,-1.2507281702 C,0,-1.0366755762,1.7953119582,-0.8434566049 HF $=-2023.0868605$

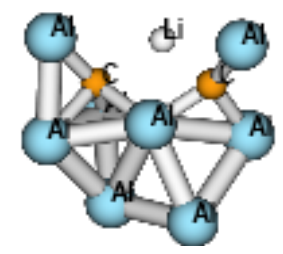

Al,0,0.2212709578,-0.1661716064,-0.4037340429 Al,0,0.5242736566,2.1059156831,-2.1472322774 Al,0,2.3894306686,1.488150014,-0.0544098826 
Al,0,3.414751692,-1.7161359408,-0.0198933957

Al,0,-1.6021572498,-2.7831279778,1.1853510861 Al,0,-1.6730814597,1.814472837,-0.7895598541 Al,0,-1.4718192249,0.8742013034,1.7678981584 Al,0,-2.4866485447,-0.7710839565,-0.5418946914 C,0,-1.0415052593,-0.9260504635,1.00965457 C,0,2.0731190242,-0.4225071412,0.2109939973 Li,0,0.9006836547,-0.9698396668,1.9070940974 HF $=-2023.0981643$

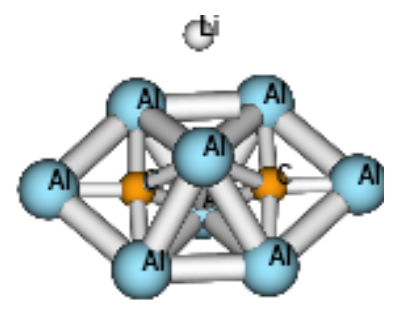

Al,0,0.2431669441,-1.4473153874,-1.9287466669 Al,0,-1.3592228411,0.3468850704,-0.5157315269 Al,0,-1.0856732886,-1.3159838004,1.8993374679 Al,0,-0.907005077,-3.3896904505,-0.0858509428 Li,0,2.9515860544,-0.6022200146,-1.7632226799 Al,0,0.8260926103,3.3945253017,0.2904409147 Al,0,1.2626226106,-0.3203852384,0.4610216059 Al,0,0.8844425101,1.3604893712,-1.7997228432 Al,0,-0.4577088328,1.4923177702,2.0247672611 C, $0,0.247073173,1.4825880567,0.1369941272$ C,0,-0.4374145772,-1.4433037624,-0.0039992052 $\mathrm{HF}=-\mathbf{2 0 2 3 . 1 1 9 7 3 6 4}$

Al,0,-2.9062337427,-1.1477156286,0.1747960517 Al,0,1.8945793849,1.3166074554,-0.2549115214 Al,0,-2.7613553366,1.3793685859,-0.8471347015 Al,0,-0.5188099769,2.1382646244,0.3716430211 Al,0,-0.5825787304,-0.2278245618,1.7934164427 Al,0,-0.6024591239,-2.1245372591,-0.6022777957 Al,0,1.7737026653,-1.3837578372,0.0911865918 Al,0,4.1746149809,-0.1248169177,0.07542404 Li,0,-0.2239253708,0.6227450986,-2.1961464146 C,0,0.2644310681,0.0076474636,-0.2555821663 C,0,-1.1739653107,0.0588716544,-0.3843192388 HF $=-2023.1102687$ 


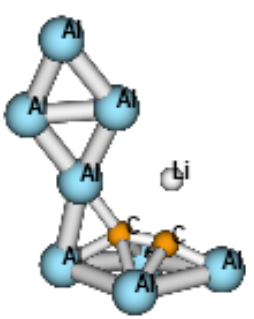

Al,0,-1.5047313828,-2.2610710773,0.0943463668 Al,0,3.3084996224,-1.3864450899,-0.015261927 Al,0,-2.6322858366,-0.1385680164,1.7103535493 Al,0,5.1547635586,0.5062344412,-0.1027724445 $\mathrm{Al}, 0,-2.6870802523,-0.2386167435,-1.6081781279$ Al,0,0.7745618084,-0.7074237148,0.0083083629 Al,0,2.5316384227,1.2242675144,-0.0795996987 Li,0,-0.1696594351,1.910964639,-0.0537176185 Al,0,-3.4081342133,2.233745014,-0.0110950837 C,0,-1.2324762193,-0.2401782242,0.0290377761 C,0,-2.0133628051,0.9484308616,0.0062688728 $\mathrm{HF}=-\mathbf{2 0 2 3 . 0 9 7 4 5 8 8}$

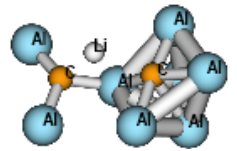

Al,0,0.4672736152,-0.8046686354,0.0215391118 Al,0,0.0650818474,1.7602811354,-1.496599668 Al,0,3.1362089683,0.5764072025,-0.9388360695 Al,0,3.0915656781,-1.4100918594,1.8769628824 Al,0,-1.7821147668,-2.2113046087,0.0516220297 Al,0,-2.5647730062,1.7944914169,-0.1031895961 Al,0,-1.7156425254,0.018650353,1.7166998961 Al,0,-1.6694710495,-0.2681516159,-1.7244360919 C,0,2.3103438575,-0.3223009638,0.5523984886 Li,0,1.0912433777,1.342301748,1.1777412187 C,0,-0.7502445289,0.8306544148,0.1505788306 $\mathrm{HF}=-\mathbf{2 0 2 3 . 1 1 2 8 7 5 1}$

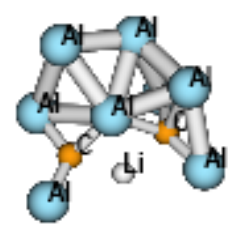

Al,0,0.2212709578,-0.1661716064,-0.4037340429 Al,0,0.5242736566,2.1059156831,-2.1472322774 Al,0,2.3894306686,1.488150014,-0.0544098826 Al,0,3.414751692,-1.7161359408,-0.0198933957 Al,0,-1.6021572498,-2.7831279778,1.1853510861 Al,0,-1.6730814597,1.814472837,-0.7895598541 Al,0,-1.4718192249,0.8742013034,1.7678981584 
Al,0,-2.4866485447,-0.7710839565,-0.5418946914

C,0,-1.0415052593,-0.9260504635,1.00965457

C,0,2.0731190242,-0.4225071412,0.2109939973

Li,0,0.9006836547,-0.9698396668,1.9070940974

HF $=-2023.0981643$

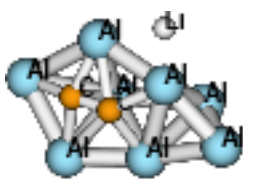

Al,0,0.6356134134,0.0678124499,1.3967225716

Al,0,-1.0234522883,-1.5771245526,-0.2139032951 Al,0,1.6018478519,-2.1474776181,-0.2128980304 Al,0,1.3357093915,2.0405654573,-0.5423509076 Al,0,-1.164389879,1.0945475478,-0.9521184987 Al,0,3.5068825737,0.1083329478,0.2835728404 Al,0,-3.4503841037,-0.5671354297,-0.8032880171 C,0,1.7927725186,-0.037364675,-0.6278532737 Li,0,-0.5021037116,2.6507475987,1.4882146514 $\mathrm{Al}, 0,-2.3573617599,0.461887944,1.386958225$ C,0,0.4419380714,-0.1643947415,-0.858759643 HF $=-\mathbf{2 0 2 3 . 1 0 5 8 5 8 7}$
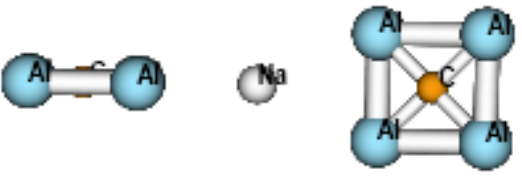

C,0,0.,-3.1729190725,3.1729190725

Al,0,-0.9980648715,-3.4496900636,4.8611462652 Al,0,-0.9856434652,-1.4862835111,2.8801734487 Al,0,0.9856434652,-2.8801734487,1.4862835111 Al,0,0.9980648715,-4.8611462652,3.4496900636 $\mathrm{Na}, 0,0 ., 0 ., 0$.

Al,0,0.9856290271,2.8801836579,-1.4862733018 Al,0,-0.9856290271,1.4862733018,-2.8801836579 Al,0,0.9980502515,4.8611566031,-3.4496797257 Al,0,-0.9980502515,3.4496797257,-4.8611566031 C,0,0.,3.1729190725,-3.1729190725 HF $=-2177.9224773$
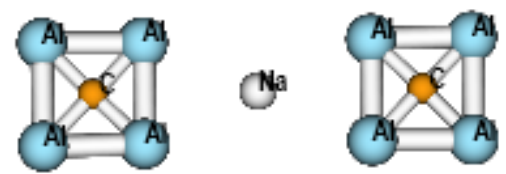

C,0,0.,-3.2030205583,3.2030205583 Al,0,0.,-3.1842914721,5.1834923048 Al,0,0.,-1.2267340081,3.1978136417 
Al,0,0.,-3.1978136417,1.2267340081

Al,0,0.,-5.1834923048,3.1842914721

$\mathrm{Na}, 0,0 ., 0 ., 0$.

$\mathrm{Al}, 0,0 ., 3.1978136417,-1.2267340081$

$\mathrm{Al}, 0,0 ., 1.2267340081,-3.1978136417$

Al,0,0.,5.1834923048,-3.1842914721

Al,0,0.,3.1842914721,-5.1834923048

C,0,0.,3.2030205583,-3.2030205583

HF $=-2177.9198901$

Al,0,1.4005878196,1.3949218304,3.0589905136 Al,0,-1.3949288578,1.4006467347,3.0589719355 Al,0,-1.4006601907,-1.3948787492,3.058980346 Al,0,1.3948724451,-1.4006040368,3.0590159829 Al,0,1.394896469,1.4006112418,-3.0592926935 Al,0,-1.3948471705,-1.4006459458,-3.0593220958 Al,0,1.4007657126,-1.3949361964,-3.0585454943 Al,0,-1.4007031146,1.3949020062,-3.0585520452 C, $0,-0.0000284708,0.0000097855,2.6990413507$ C, $0,0.0000257493,-0.0000276455,-2.6993736477$ $\mathrm{Na}, 0,0.0000214422,-0.0000102132,-0.0001100051$ HF $=-2177.8867144$

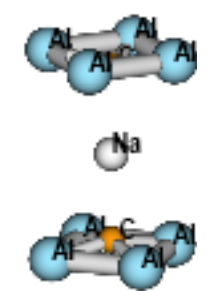

Al,0,0.000987678,1.9767896466,3.0607136441 Al,0,-1.9767898769,0.001003326,3.0607181354 Al,0,-0.0010035306,-1.9767725763,3.0607240262 Al,0,1.9767741898,-0.0009860434,3.0607269871 Al,0,1.3970172666,1.3986217248,-3.0606304357 Al,0,-1.3970006715,-1.3986393268,-3.0606301087 Al,0,1.3984194784,-1.3972365921,-3.0606222302 Al,0,-1.3984030579,1.3972191836,-3.0606379064 C, $0,-0.0000070529,0.0000076172,2.6999430567$ C,0,0.0000072119,-0.0000076475,-2.6998607182 $\mathrm{Na}, 0,-0.0000018316,0.0000007935,-0.0004728622$ HF $=-\mathbf{2 1 7 7 . 8 8 6 7 5 8}$ 

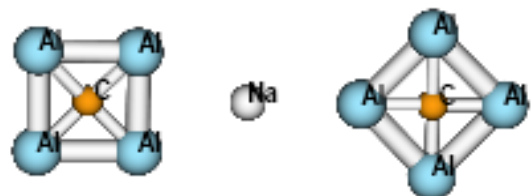

C,0,0.,-3.3517293132,3.3517293394^M Al,0,0.,-3.3360109678,5.3335048368^M Al,0,0.,-1.378310729,3.3557394874^M Al,0,0.,-3.3557394766,1.3783107553^M Al,0,0.,-5.3335048106,3.3360110096^M $\mathrm{Na}, 0,0 .,-0.2287302682,0.22873027 \wedge \mathrm{M}$ $\mathrm{C}, 0,0 ., 3.3863750959,-3.3863751225^{\wedge} \mathrm{M}$ $\mathrm{Al}, 0,0 ., 2.0030132852,-2.0030133009 \wedge \mathrm{M}$ Al,0,0.,4.7892966476,-4.7892966851^M Al,0,0.,4.8003738274,-1.9884328964^M Al,0,0.,1.9884328588,-4.800373843^M $\mathrm{HF}=-2177.9211701$

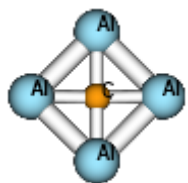

(N)

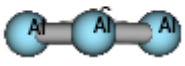

C,0,-3.6323588996,0.,0.

Al,0,-3.9603095639,0.,1.9811405999 Al,0,-3.9490924075,1.9813443188,0. Al,0,-3.9603095639,0.,-1.9811405999 Al,0,-3.9490924075,-1.9813443188,0. Na,0,-0.9787010726,0.,0. Al,0,2.1407637505,0.,0. Al,0,6.0792440258,0.,0. C,0,4.0960212369,0.,0. Al,0,4.1064649207,0.,1.9889607792 Al,0,4.1064649207,0.,-1.9889607792 $\mathrm{HF}=-2177.9067065$
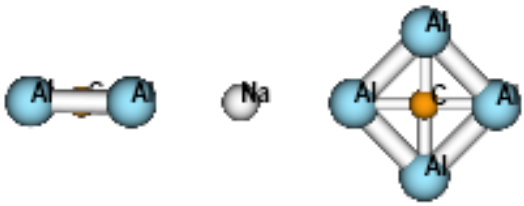

C,0,0.0000003127,-3.3390992284,3.3390996707 Al,0,-0.9976303518,-3.6180010992,5.0288439452 Al,0,-0.9884929164,-1.6561727849,3.0540930139 Al,0,0.9884933575,-3.054092702,1.6561730969 
Al,0,0.9976311615,-5.0288433726,3.6180016719

$\mathrm{Na}, 0,0.0000000212,-0.2259392897,0.2259393196$ Al,0,1.4055209991,4.3761067389,-2.3883707543 Al,0,-0.0000001864,1.9907457992,-1.9907460629 Al,0,-0.0000004473,4.7768903276,-4.7768909603 Al,0,-1.4055216325,2.388370043,-4.3761069236 C,0,-0.000000316,3.3741482013,-3.3741486482 HF $=-\mathbf{2 1 7 7 . 9 2 2 5 9 7 9}$

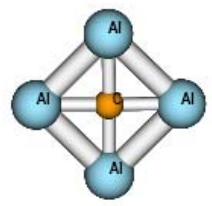

(1)

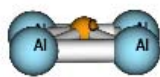

C,0,-3.6329550106,0.,0.

Al,0,-3.9544629485,0.756657862,1.8310641202 Al,0,-3.9544629485,1.8297957584,-0.7597199582 Al,0,-3.9544629485,-0.756657862,-1.8310641202 Al,0,-3.9544629485,-1.8297957584,0.7597199582 $\mathrm{Na}, 0,-0.9791515742,0 ., 0$.

$\mathrm{Al}, 0,2.1406222775,0.0$.

$\mathrm{Al}, 0,6.0791579954,0 ., 0$.

C,0,4.0958970683,0.,0.

Al,0,4.1064594132,-0.7611551285,1.8375910445 Al,0,4.1064594132,0.7611551285,-1.8375910445 HF $=-2177.9066838$

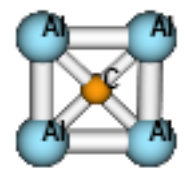

Wa

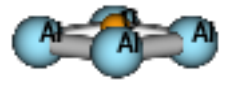

C,0,-3.3802049395,0.,0.

Al,0,-3.7160482134,0.,1.9795413521

Al,0,-3.6982055675,1.9792365044,0.

Al,0,-3.7160482134,0.,-1.9795413521

Al,0,-3.6982055675,-1.9792365044,0.

$\mathrm{Na}, 0,-0.6621298063,0 ., 0$.

Al,0,2.4048273754,0.,-1.3935129127

$\mathrm{Al}, 0,5.1912528061,0 ., 1.4146091727$ 
C,0,3.806195182,0.,0.

Al,0,2.4048273754,0.,1.3935129127

Al,0,5.1912528061,0.,-1.4146091727

HF $=-2177.9059037$
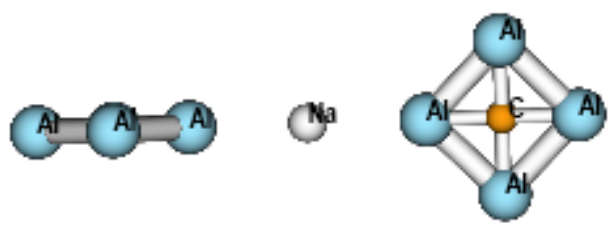

$\mathrm{Na}, 0,0 ., 0 ., 0$.

Al,0,1.4064260895,4.549343984,-2.5603279854

Al,0,-0.0000002036,2.1689611071,-2.1689613934

Al,0,-0.0000004647,4.9502756268,-4.9502762804

Al,0,-1.406426757,2.5603272527,-4.5493441901

C,0,-0.000000333,3.5475354196,-3.547535888

Al,0,0.0000002036,-2.1689611071,2.1689613934

Al,0,0.0000004647,-4.9502756268,4.9502762804

C,0,0.000000333,-3.5475354196,3.547535888

Al,0,-1.4064466987,-2.5603419576,4.5493297492

Al,0,1.4064473661,-4.5493292791,2.5603424263

HF $=-2177.921247$
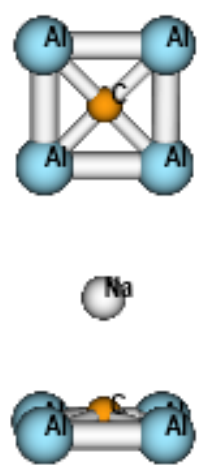

C,0,-3.3804394257,0.,0.

Al,0,-3.7073102482,0.7552205225,1.8295733392 Al,0,-3.7073102482,1.8277252697,-0.759682157

Al,0,-3.7073102482,-0.7552205225,-1.8295733392

Al,0,-3.7073102482,-1.8277252697,0.759682157

$\mathrm{Na}, 0,-0.6618612155,0 ., 0$.

Al,0,2.4049682833,0.5332875179,-1.2874699533

Al,0,5.1913792125,-0.5413661486,1.3069734931

C,0,3.8063679899,0.,0.

Al,0,2.4049682833,-0.5332875179,1.2874699533

Al,0,5.1913792125,0.5413661486,-1.3069734931

HF $=-\mathbf{2 1 7 7 . 9 0 5 8 5 0 2}$ 


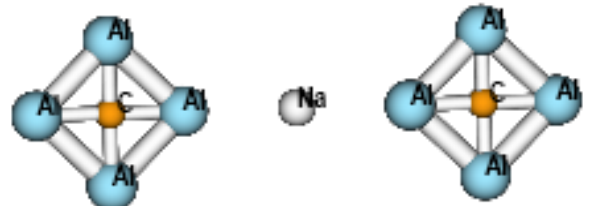

Na, $0,0 ., 0 ., 0$.

Al,0,1.4065041712,4.5556624803,-2.5665360598

Al,0,-0.0000002033,2.1752850672,-2.1752853547

Al,0,-0.0000004633,4.9567217196,-4.9567223746

Al,0,-1.4065048369,2.5665353263,-4.555662688

C,0,-0.0000003322,3.5538984345,-3.5538989041

Al,0,0.0000002033,-2.1752850672,2.1752853547

Al,0,0.0000004633,-4.9567217196,4.9567223746

C,0,0.0000003322,-3.5538984345,3.5538989041

Al,0,1.4065048369,-2.5665353263,4.555662688

Al,0,-1.4065041712,-4.5556624803,2.5665360598

$\mathrm{HF}=-\mathbf{2 1 7 7 . 9 2 0 3 6 1 6}$

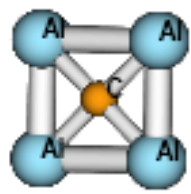

Na

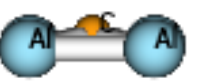

Al,0,2.3728877955,-1.3826582479,-0.4516829508 Al,0,5.153770906,-1.5180607013,-0.3444909935 Al,0,2.4387584674,1.2046881437,0.5862452296 Al,0,5.2205843763,1.106216067,0.7092724598 Al,0,-3.7430168253,0.1906214297,1.8615889236 Al,0,-3.6415756822,2.1210993608,-0.1605809816 Al,0,-3.6542248204,0.0935934158,-2.096127421 Al,0,-3.782162616,-1.8358674802,-0.0743391023 C,0,3.8044622748,-0.147712683,0.1250787523 C,0,-3.3811522123,0.130958467,-0.1097992813 $\mathrm{Na}, 0,-0.662285563,0.0332099506,-0.043653087$ $\mathbf{H F}=-\mathbf{2 1 7 7 . 9 0 5 8 5 8 9}$

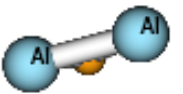

(4)

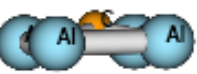


Al,0,2.6547297014,-0.7602695996,-1.8401535232 Al,0,3.716820798,-1.86908695,0.4911607215 Al,0,2.705972709,1.7972816751,-0.629089114 Al,0,3.7666196858,0.6621789696,1.6899147737 Al,0,-2.9382279329,0.1342409248,2.0707231882 Al,0,-3.2872639959,1.9957655826,0.0128029044 Al,0,-3.4390301016,-0.0860110756,-1.8459703328 Al,0,-3.0918754146,-1.9489654656,0.2104369289 C,0,2.9871130244,-0.0706028931,-0.0032700746 C,0,-2.8274970618,0.0385020695,0.0663078223 $\mathrm{Na}, 0,-0.1907624197,0.1059874678,-0.223268963$ HF $=-2177.8845439$

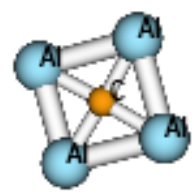

No

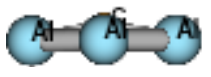

C, $0,-3.4709329546,-0.04094815,-0.0832680868$ Al,0,-3.832290646,-0.0902385336,1.8911127026 Al,0,-3.8111011058,1.9349645966,-0.0413045216 Al,0,-3.7769914683,0.0012150202,-2.0676461207 Al,0,-3.7669403777,-2.0238010467,-0.133521325 $\mathrm{Na}, 0,-0.780885685,-0.0112617831,-0.0546845575$ Al,0,2.2490375542,-0.0219086953,-0.9309383819 Al,0,5.6136113768,0.114706769,1.1171216534 C,0,3.9116979657,0.0472270664,0.1173398342 Al,0,2.869100297,0.1182270419,1.7990297074 Al,0,4.9128937905,-0.0265339123,-1.6033075875 HF $=-\mathbf{2 1 7 7 . 9 0 5 4 0 5 7}$

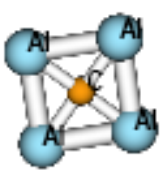

Na

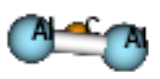

C,0,3.4807115665,1.2787761972,0.

Al,0,3.703971833,2.6894060336,1.4000968156 Al,0,3.6446759841,-0.1055280956,1.4150390861 Al,0,3.6446759841,-0.1055280956,-1.4150390861 Al,0,3.703971833,2.6894060336,-1.4000968156 
$\mathrm{Na}, 0,0.5326482961,-0.1739339355,0$.

Al,0,-2.7184523696,0.4178205143,0.

Al,0,-4.7928450832,-2.9479711682,0.

C,0,-3.7577682317,-1.2605671451,0.

Al,0,-5.4281934644,-0.1941664243,0.

$\mathrm{Al}, 0,-2.0806348142,-2.3046681072,0$.

HF $=-2177.9032124$ 


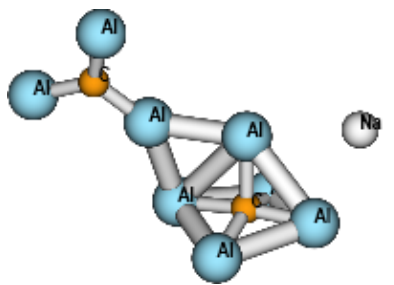

Al,0,-0.8045542043,-1.4791926001,0.6127191031 Al,0,-2.2235600897,-0.9675870289,-1.7616592063 Al,0,1.5728687887,-0.5949700868,-0.1292710744 Al,0,-3.1745801219,1.495928538,-0.7064932062 Al,0,4.5459074333,0.1404277093,-1.2334780947 Al,0,-2.3702948168,0.339066853,1.9053796305 Al,0,-0.2647470725,1.3458858183,-0.1574978866 Na,0,-2.2507357826,3.4841587342,1.654480616 Al,0,3.9288010004,-2.7767143705,0.2744482552 C,0,3.3995235923,-1.0746840753,-0.3520661989 C,0,-1.8944966448,0.0975625926,-0.0901345586 $\mathrm{HF}=-2177.891662$

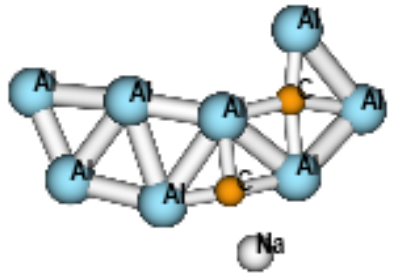

Al,0,3.8094905719,0.5712553797,-0.5052935338 Al,0,4.5144757432,-1.8701612596,-0.0889830864 Al,0,1.4053700927,1.5515675541,-0.3794609088 Al,0,2.011965771,-1.2128937893,0.3609244271 Al,0,-4.2619230158,-0.4874812777,-0.6655319408 Al,0,-2.7530867874,-2.6588683036,0.3999490604 Al,0,-2.276888282,1.1974668995,-0.5001557056 C,0,-0.4181407093,1.5322183595,0.0457616662 $\mathrm{Na}, 0,-0.8319057713,3.6054228964,1.2341956309$ Al,0,-0.4610766486,-0.470281746,0.3242118641 C,0,-2.3647415075,-0.8201344931,-0.0240507047 HF $=-2177.8570404$

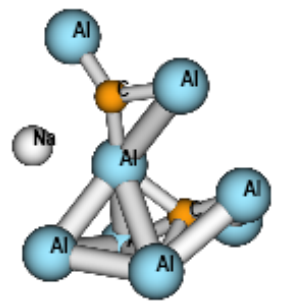

Al,0,1.4529201932,0.0030434456,1.9779724619 Al,0,1.8320951706,-1.9964379179,-0.0099405743 
Al,0,-0.5514340818,0.2678627084,-0.1874478902 Al,0,-0.5845806461,-3.0844954358,1.2630390861 Al,0,1.1777689125,-0.303300014,-2.6645076532 Al,0,-0.4236314447,1.9745415561,1.9867400471 Al,0,-1.3561449916,-0.5840585116,2.1199333383 $\mathrm{Na}, 0,0.177852093,3.0789199687,-0.94526625$ Al,0,-1.6744133777,1.326181767,-3.1780264878 C,0,-0.2596130842,0.825135046,-2.0250633684 C,0,0.2096281563,-1.2770531171,0.9245664496 HF $=-2177.8765436$

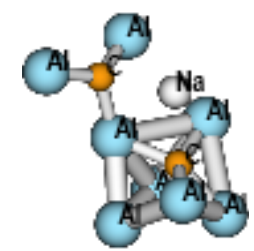

Al,0,0.5704647956,-0.5997530078,-0.5854389342 Al,0,-0.9596790823,2.2935650885,-0.9187904463 $\mathrm{Al}, 0,3.7706394228,-0.5168400431,-1.0263496347$ Al,0,2.1790464956,-1.5536897016,1.8872350792 Al,0,-2.1381687127,-1.532525878,-0.3033979043 Al,0,-2.9979974463,0.9529982372,0.2088861093 Al,0,-1.0528637388,-0.3910101203,1.9046671018 Al,0,-1.2810656928,-0.023806877,-2.2639230205 C,0,2.3548784173,-0.6237879424,0.2124087385 Na,0,1.4783053056,1.6532329433,1.1060770731 C,0,-0.9275862334,0.5634958676,0.1368585351 $\mathbf{H F}=-\mathbf{2 1 7 7 . 8 8 1 1 0 2 3}$

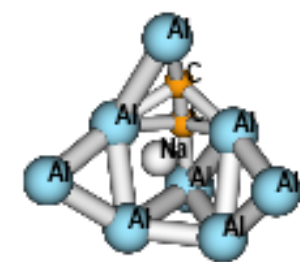

Al,0,-1.7023945848,1.0709663929,-1.0406513294 Al,0,-3.1007577004,-1.1761607259,-1.071165139 Al,0,-0.5189132617,2.0278722984,1.5144542717 Al,0,-1.3569754347,-1.0126728219,0.921682719 Al,0,0.619700912,2.2538685159,-1.0778286468 Al,0,2.83993589,0.9492863741,-1.6429386534 Al,0,0.9749667476,-2.8209492977,1.4945440373 Al,0,1.689552921,0.1977520287,0.6637960491 C,0,0.0478959274,0.2560646546,1.983595951 $\mathrm{Na}, 0,0.3670886196,-1.3425405334,-1.8752774111$ C,0,0.4813580441,-1.0229929999,1.9703104676 


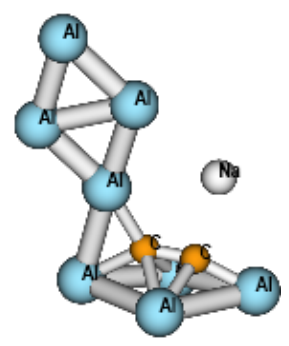

Al,0,1.2579498491,2.4866066673,-0.0130563614 Al,0,-3.2874204795,1.4129011416,-0.0172820206 Al,0,2.6412068434,0.5189758406,1.6729291676 Al,0,-5.2174528153,-0.391979207,-0.0108351743 Al,0,2.6488183293,0.4968794597,-1.6664586673 Al,0,-0.8201219955,0.5724367633,-0.0053935485 Al,0,-2.6445635354,-1.2597629249,0.0016918386 $\mathrm{Na}, 0,0.2624879045,-2.384499218,0.0159125424$ Al,0,3.6645705316,-1.7334753774,0.0203406326 C,0,1.2280591043,0.4677993581,0.0002939587 C,0,2.0975751605,-0.6518125788,0.0096720025 $\mathbf{H F}=-\mathbf{2 1 7 7 . 8 6 2 7 2 4 1}$

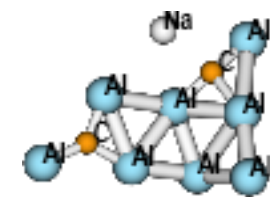

Al,0,2.3750558969,-0.4888618798,-0.2073936137 Al,0,0.7811433478,-1.6130188432,1.4624016181 Al,0,-0.262839751,-0.1242157785,-0.6855702224 Al,0,-1.1110172504,-3.3136002317,3.7645238821 Al,0,1.1651849713,1.9869459294,-1.4238842906 Al,0,3.3216076655,1.9465383401,0.1177624367 Al,0,-2.0155627709,-1.2667054818,1.0623380346 $\mathrm{Na}, 0,-3.0258953871,1.1125358414,-1.0070486985$ $\mathrm{Al}, 0,-0.9852147786,2.3258587266,-3.4355190878$ C,0,-0.6715488008,1.3617371961,-1.8375673406 C,0,-0.8624172056,-2.2160912641,2.2653959809 HF $=-2177.8631928$

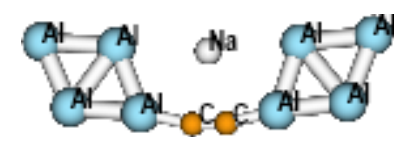

Al,0,3.3736304182,1.1626438994,0.0004562793^M $\mathrm{Al}, 0,2.5001316445,-1.2657857123,0.0007059279 \wedge \mathrm{M}$ $\mathrm{Al}, 0,6.0956392028,1.3795737633,0.0001857345^{\wedge} \mathrm{M}$ $\mathrm{Al}, 0,5.0850161676,-1.0479091045,0.0004072507 \wedge \mathrm{M}$ 
$\mathrm{Al}, 0,-2.5213815327,-1.2235856519,-0.0042651753^{\wedge} \mathrm{M}$ $\mathrm{Al}, 0,-3.3534275113,1.2194608598,-0.0002692896 \wedge \mathrm{M}$ $\mathrm{Al}, 0,-6.0711832018,1.4822436009,0.0037801134 \wedge \mathrm{M}$ $\mathrm{Al}, 0,-5.1018047975,-0.9618864316,0.0009884529 \wedge \mathrm{M}$ $\mathrm{Na}, 0,0.0078568593,0.9670679495,-0.0004392907 \wedge \mathrm{M}$ C,0,0.6137985208,-1.6982314684,-0.00074296^M $\mathrm{C}, 0,-0.642546941,-1.6883627558,-0.0027618101 \wedge \mathrm{M}$

\section{$\mathbf{H F}=-\mathbf{2 1 7 7 . 8 3 9 6 0 7 6}$}

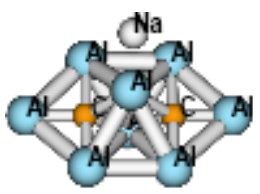

Al,0,1.42483314,-1.3632540189,-1.497648158 Al,0,-0.0916739512,-1.5710920471,0.7599297285 Al,0,1.4507680642,0.6613251493,2.0876665665 Al,0,3.475631876,-0.5002779369,0.3594754218 $\mathrm{Na}, 0,0.1930344332,3.214347085,-2.4919713257$ Al,0,-3.5162000473,-0.2492095572,0.2393581443 Al,0,0.0564308814,0.8972324859,-0.5544285847 Al,0,-1.4712591302,-1.2061376945,-1.5759645513 Al,0,-1.476773873,0.8127328933,2.0133432616 C,0,-1.5116499104,-0.1447352142,0.2642933763 C,0,1.4789467032,-0.2910928684,0.3355684252 HF $=-2177.8946298$ 
Al,0,-1.046027759,0.205916368,1.8470815514 $\mathrm{Al}, 0,0.2580207592,-2.400261839,0.0398092349$ Al,0,1.6442444702,-0.4004475422,1.5426941167 Al,0,0.6408559266,1.8663187153,-0.0340447325 Al,0,-1.0638183611,0.1407772774,-1.8452529199 $\mathrm{Al}, 0,3.238847065,1.1784123979,-0.0322083074$ Al,0,1.6297138086,-0.4514260311,-1.5414973062 C,0,0.0452660062,-0.1937237864,0.0016235272 $\mathrm{Na}, 0,-2.2971593599,2.6450045971,-0.0358831745$ Al,0,-2.8087937216,-1.897050123,0.0439403265 C,0,-1.2353985868,-0.8469696256,0.0196980381 $\mathbf{H F}=-\mathbf{2 1 7 7 . 8 8 6 0 6 7 3}$

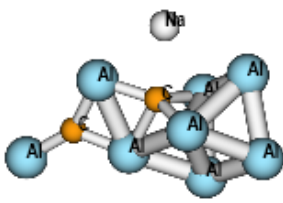

Al,0,4.7351989126,-1.0264858616,-0.1190625332 Al,0,2.0235392938,1.4371253313,-0.1022544054 Al,0,-0.9201809306,0.1959987551,1.7024410084 $\mathrm{Al}, 0,-0.9662726949,-2.2284727738,0.50295448$ Al,0,-2.8954320085,1.2213419516,0.2316969239 Al,0,-1.0897307212,-0.0474231778,-1.3591844223 $\mathrm{Na}, 0,-0.4847124391,3.1677089974,-0.1397143987$ Al,0,1.3293787223,-1.0724766282,-0.028991512 C,0,3.0600316833,-0.2082737293,-0.0854554881 C,0,0.1459778815,0.6580909942,-0.0077670299 Al,0,-3.2860560011,-1.3675847167,-0.666353886 $\mathbf{H F}=-\mathbf{2 1 7 7 . 8 6 6 4 8 7 8}$ 

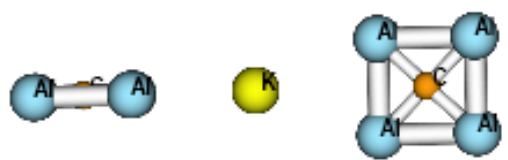

C,0,0.,-3.5064221094,3.5064221094^M

Al,0,-0.9941814251,-3.7930183484,5.1989826079^M

Al,0,-0.9902538101,-1.82294531,3.2233551644^M

$\mathrm{Al}, 0,0.9902538101,-3.2233551644,1.82294531 \wedge \mathrm{M}$

$\mathrm{Al}, 0,0.9941814251,-5.1989826079,3.7930183484 \wedge \mathrm{M}$

$\mathrm{K}, 0,0 ., 0 ., 0 . \wedge \mathrm{M}$

$\mathrm{Al}, 0,0.9902393045,3.2233654214,-1.822935053^{\wedge} \mathrm{M}$

$\mathrm{Al}, 0,-0.9902393045,1.822935053,-3.2233654214 \wedge \mathrm{M}$

$\mathrm{Al}, 0,0.994166862,5.1989929056,-3.7930080507 \wedge \mathrm{M}$

$\mathrm{Al}, 0,-0.994166862,3.7930080507,-5.1989929056 \wedge \mathrm{M}$

C,0,0.,3.5064221094,-3.5064221094^M

HF $=-\mathbf{2 6 1 5 . 5 3 4 5 5 2 7}$
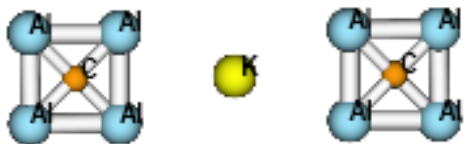

C,0,0.,-3.5265294175,3.5265294175^M

$\mathrm{Al}, 0,0 .,-3.5194732311,5.5101132347 \wedge \mathrm{M}$

$\mathrm{Al}, 0,0 .,-1.552082144,3.5321986353 \wedge \mathrm{M}$

$\mathrm{Al}, 0,0 .,-3.5321986353,1.552082144 \wedge \mathrm{M}$

$\mathrm{Al}, 0,0 .,-5.5101132347,3.5194732311^{\wedge} \mathrm{M}$

$\mathrm{K}, 0,0 ., 0 ., 0 . \wedge \mathrm{M}$

$\mathrm{Al}, 0,0 ., 3.5321986353,-1.552082144 \wedge \mathrm{M}$

$\mathrm{Al}, 0,0 ., 1.552082144,-3.5321986353 \wedge \mathrm{M}$

Al,0,0.,5.5101132347,-3.5194732311^M

$\mathrm{Al}, 0,0 ., 3.5194732311,-5.5101132347 \wedge \mathrm{M}$

C,0,0.,3.5265294175,-3.5265294175^M

$\mathrm{HF}=-\mathbf{2 6 1 5 . 5 3 3 0 1 6}$

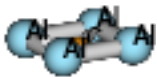

C.

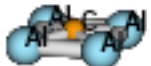

Al,0,-0.000486411,1.9826963215,3.572174178

Al,0,-1.9826940282,-0.0004589185,3.5721973533

Al,0,0.0004582207,-1.9826742758,3.5721715412

Al,0,1.9826722456,0.000485001,3.5721886643

Al,0,1.4023131925,1.4015726035,-3.5723713128

Al,0,-1.4022982023,-1.4015889543,-3.5723744286

Al,0,1.401669367,-1.4022404887,-3.5723712133

Al,0,-1.4016506562,1.4022180447,-3.5723920458 
C,0,-0.000008579,0.0000093825,3.3227286278

C,0,0.000011614,-0.0000114936,-3.3250700312

K,0,0.0000101751,-0.0000057193,0.0012712027

$\mathbf{H F}=-\mathbf{2 6 1 5 . 5 0 4 3 7 1 7}$
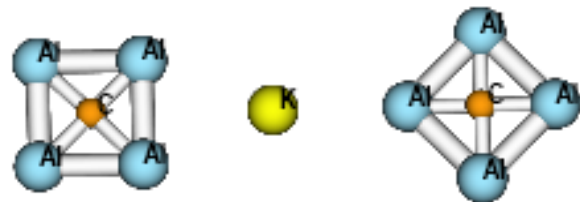

C,0,0.,-3.6653843318,3.6653843605^M Al,0,0.,-3.6615456678,5.6503420026^M $\mathrm{Al}, 0,0 .,-1.6936737491,3.6796322381^{\wedge} \mathrm{M}$ $\mathrm{Al}, 0,0 .,-3.6796322248,1.6936737779 \wedge \mathrm{M}$ $\mathrm{Al}, 0,0 .,-5.650341974,3.661545712^{\wedge} \mathrm{M}$ $\mathrm{K}, 0,0 .,-0.2099864112,0.2099864129 \wedge \mathrm{M}$ C,0,0.,3.7334512512,-3.7334512804^M $\mathrm{Al}, 0,0 ., 2.3474723707,-2.3474723891 \wedge \mathrm{M}$ $\mathrm{Al}, 0,0 ., 5.1360852678,-5.136085308^{\wedge} \mathrm{M}$ $\mathrm{Al}, 0,0 ., 5.144079231,-2.3330445017 \wedge \mathrm{M}$ $\mathrm{Al}, 0,0 ., 2.3330444614,-5.1440792492 \wedge \mathrm{M}$ HF $=-\mathbf{2 6 1 5 . 5 3 2 0 6 8 1}$
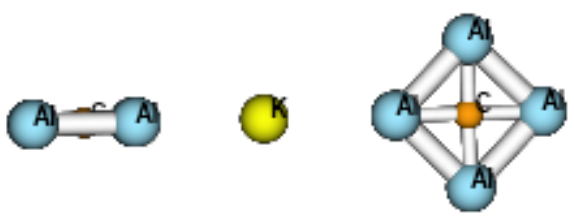

C,0,0.0000003425,-3.6573953209,3.6573958054^M $\mathrm{Al}, 0,-0.9943057571,-3.9448380727,5.350979386^{\wedge} \mathrm{M}$ Al,0,-0.9931287059,-1.9765206102,3.3809968246^M Al,0,0.9931292076,-3.3809964698,1.9765209651^M $\mathrm{Al}, 0,0.9943066275,-5.3509787704,3.9448386884 \wedge \mathrm{M}$ $\mathrm{K}, 0,0.0000000195,-0.2087736964,0.2087737241^{\wedge} \mathrm{M}$ $\mathrm{Al}, 0,1.4054576106,4.7237485826,-2.7361022432 \wedge \mathrm{M}$ $\mathrm{Al}, 0,-0.0000002191,2.3394947182,-2.3394950281^{\wedge} \mathrm{M}$ $\mathrm{Al}, 0,-0.0000004801,5.1277195749,-5.1277202541^{\wedge} \mathrm{M}$ $\mathrm{Al}, 0,-1.4054583091,2.7361014858,-4.7237488134 \wedge \mathrm{M}$ C,0,-0.0000003488,3.7254294095,-3.725429903^M HF $=-2615.5329401$
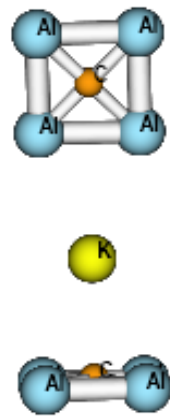
C,0,-3.967780903,0.,0.

Al,0,-4.1762833285,0.7582634706,1.8340378295

Al,0,-4.1762833285,1.8330338303,-0.7606873392

Al,0,-4.1762833285,-0.7582634706,-1.8340378295

Al,0,-4.1762833285,-1.8330338303,0.7606873392

$\mathrm{K}, 0,-0.601314504,0 ., 0$.

$\mathrm{Al}, 0,2.958056093,0.5359006492,-1.2937786103$

Al,0,5.7456537015,-0.5391242291,1.3015610207

C,0,4.3503232366,0.,0.

$\mathrm{Al}, 0,2.958056093,-0.5359006492,1.2937786103$

Al,0,5.7456537015,0.5391242291,-1.3015610207

$\mathbf{H F}=-\mathbf{2 6 1 5 . 5 2 0 3 3 0 1}$

(a)

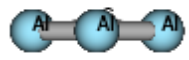

C,0,-4.1635433556,0.,0.

$\mathrm{Al}, 0,-4.3807462176,0 ., 1.9853236194$

$\mathrm{Al}, 0,-4.3705072168,1.9853270749,0$.

Al,0,-4.3807462176,0.,-1.9853236194

Al,0,-4.3705072168,-1.9853270749,0.

$\mathrm{K}, 0,-0.9099472804,0 ., 0$.

$\mathrm{Al}, 0,2.6846744234,0 ., 0$.

$\mathrm{Al}, 0,6.6276517396,0 ., 0$.

C,0,4.6453544911,0.,0.

$\mathrm{Al}, 0,4.6488646418,0 ., 1.9884054946$

$\mathrm{Al}, 0,4.6488646418,0 .,-1.9884054946$

$\mathrm{HF}=\mathbf{- 2 6 1 5 . 5 1 9 4 3 9 9}$

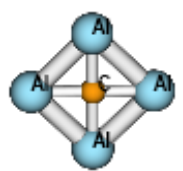

(

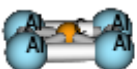

C,0,-4.168112654,0.,0.

Al,0,-4.3769585083,0.7594459671,1.8345235648

Al,0,-4.3769585083,1.8342134432,-0.7601946669

Al,0,-4.3769585083,-0.7594459671,-1.8345235648

Al,0,-4.3769585083,-1.8342134432,0.7601946669 
$\mathrm{K}, 0,-0.9082307728,0 ., 0$.

Al,0,2.6859055618,0.,0.

$\mathrm{Al}, 0,6.6282474909,0 ., 0$.

C,0,4.6460778267,0.,0.

Al,0,4.6502479382,-0.760771371,1.8366645721

$\mathrm{Al}, 0,4.6502479382,0.760771371,-1.8366645721$

\section{HF =-2615.5194535}
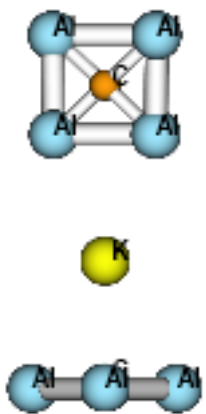

C,0,-3.9630955369,0.,0.

Al,0,-4.1837955236,0.,1.9846191164

Al,0,-4.1651587112,1.9844403049,0.

Al,0,-4.1837955236,0.,-1.9846191164

$\mathrm{Al}, 0,-4.1651587112,-1.9844403049,0$.

K,0,-0.6025895462,0.,0.

$\mathrm{Al}, 0,2.9563522485,0 .,-1.4003302307$

$\mathrm{Al}, 0,5.7439750702,0 ., 1.4088112039$

C,0,4.3486790698,0.,0.

Al,0,2.9563522485,0.,1.4003302307

$\mathrm{Al}, 0,5.7439750702,0 .,-1.4088112039$

\section{$\mathrm{HF}=-\mathbf{2 6 1 5 . 5 2 0 3 2 8 4}$}
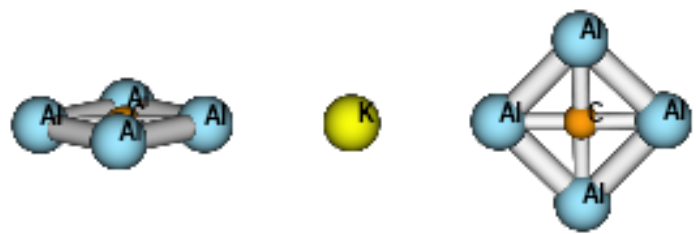

$\mathrm{K}, 0,0 ., 0 ., 0$.

Al,0,1.4064276002,4.8800725598,-2.8910544244 Al,0,-0.0000002346,2.4984842572,-2.4984845871 Al,0,-0.0000004959,5.2820850858,-5.2820857833 Al,0,-1.4064283298,2.891053648,-4.8800728095 C,0,-0.0000003642,3.8796478367,-3.879648349 Al,0,0.0000002346,-2.4984842572,2.4984845871 Al,0,0.0000004959,-5.2820850858,5.2820857833 C,0,0.0000003642,-3.8796478367,3.879648349 Al,0,-1.4064482094,-2.8910683529,4.8800583686 Al,0,1.406448939,-4.8800578548,2.8910688653 $\mathbf{H F}=-\mathbf{2 6 1 5 . 5 3 0 1 1 3 7}$ 


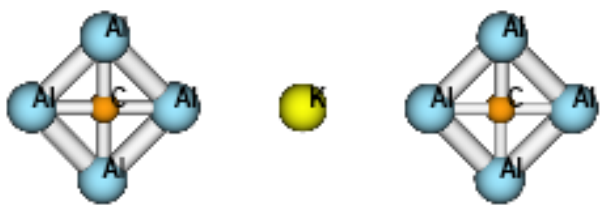

$\mathrm{K}, 0,0 ., 0 ., 0$.

Al,0,1.4064958246,4.8844344764,-2.89531986

$\mathrm{Al}, 0,-0.000000234,2.5029814483,-2.502981779$

Al,0,-0.0000004942,5.2866307041,-5.2866314027

Al,0,-1.4064965518,2.895319083,-4.8844347276

C,0,-0.0000003631,3.8842112099,-3.8842117232

$\mathrm{Al}, 0,0.000000234,-2.5029814483,2.502981779$

Al,0,0.0000004942,-5.2866307041,5.2866314027

C,0,0.0000003631,-3.8842112099,3.8842117232

$\mathrm{Al}, 0,1.4064965518,-2.895319083,4.8844347276$

$\mathrm{Al}, 0,-1.4064958246,-4.8844344764,2.89531986$

HF $=-\mathbf{2 6 1 5 . 5 2 9 5 6 0 4}$

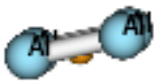

O

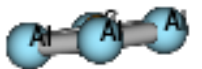

C,0,-3.3743713785,-0.2288660645,0.0046021518 Al,0,-3.5658421089,-0.3849377949,1.9868854872 Al,0,-3.997590323,1.6671264221,0.1266900657 Al,0,-3.6563706765,-0.1581950804,-1.9723581897 $\mathrm{Al}, 0,-3.2240641204,-2.2153420316,-0.1136507607$ $\mathrm{K}, 0,-0.0562258306,0.0951076175,-0.0068393678$ Al,0,3.2114008561,1.4862345576,-1.4970412044 Al,0,4.0396186078,-1.0015769804,1.4808536403 C,0,3.4266405115,0.1898640416,-0.0015841632 $\mathrm{Al}, 0,3.2070576756,1.66899902,1.3127581874$ Al,0,4.0438420881,-1.1833106197,-1.3155341446 HF $=-2615.5042606$

CAl4kCAl4-iso6-b3lan-631+gx.frq24.3 13.46015225 O

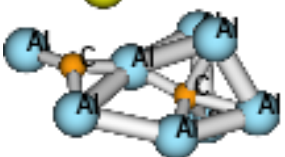

Al,0,0.3558022835,-0.8489331847,-0.0622771287 Al,0,-0.1915601614,1.8413197032,-1.6828970612 Al,0,2.6411640405,0.9271915452,-0.8907022846 Al,0,3.2074147223,-2.2100666122,0.4490449357 
Al,0,-2.0268812659,-1.5617364028,0.591965621

Al,0,-2.9512923442,1.3080544338,-1.358154907

Al,0,-1.7877354151,1.0449837803,1.0120153533

Al,0,-2.0365151789,-1.1131794463,-2.0460570288

C,0,2.2448998157,-0.5915263267,0.2136472831

$\mathrm{K}, 0,1.5271463879,0.5220346014,2.928147695$

C,0,-1.0367228523,0.2652101534,-0.8474795664

HF $=-2615.5131026$

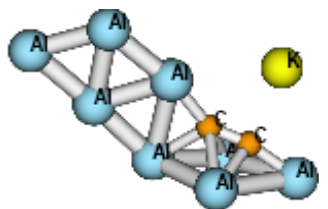

Al,0,-0.269884419,-1.881314466,0.0547271612

Al,0,-2.7483061197,-1.0005559835,0.0399749657 Al,0,1.8868582431,-1.438324049,-1.5920602879 Al,0,-5.3312786008,-0.2400026447,0.0194920896 Al,0,1.8939706423,-1.3420081866,1.6609807439 Al,0,-0.848981949,0.8242917553,-0.0212937017 Al,0,-3.3857238807,1.5320760841,-0.0300196631 $\mathrm{K}, 0,2.1793561252,2.781783792,-0.089997696$ Al,0,4.1525786166,-0.4485935953,0.0036308599 C, $0,0.8932166426,-0.0973570058,-0.00249731$ C,0,2.2821518068,-0.0570243168,-0.0059463493 HF $=-\mathbf{2 6 1 5 . 5 0 1 0 0 3 7}$

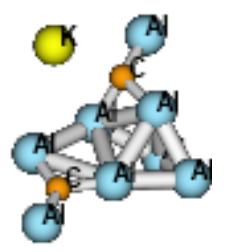

Al,0,-4.572953662,-0.1047362661,-0.3235392374 Al,0,-1.7335237643,-1.307995398,1.3861899831 Al,0,-0.0355717502,3.0563736208,-1.2765195895 Al,0,0.7520136616,-0.1163981304,1.3930943659 Al,0,1.2562420925,2.4456934106,1.1311514183 Al,0,3.8348885017,-0.7092515081,-0.009166974 Al,0,1.1208939461,0.7460702562,-1.2557265569 C,0,1.940235177,-0.5752681171,-0.114050145 K,0,0.8651184608,-3.266773447,-0.5581881687 Al,0,-1.4961590713,1.1084055128,-0.3042409116 C,0,-2.7857418703,-0.1692992125,0.2756206005 HF $=-\mathbf{2 6 1 5 . 4 9 5 2 0 8 3}$ 


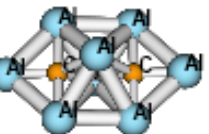

Al,0,-2.7166375105,-0.6940447444,-1.0020778145 Al,0,-1.105215077,0.2564760339,1.4185811291 Al,0,0.6671923267,-1.7852330304,1.0653814401 Al,0,-1.7655202466,-3.1417070796,0.1993790578 Al,0,0.4209928388,3.5248192377,0.1459724023 Al,0,0.1902616225,-0.0224826866,-1.0310439813 Al,0,-1.9048764543,2.1211246511,-0.8313441464 Al,0,1.4681094099,1.0273944294,1.2528337104 C,0,-0.2458861026,1.6210159439,0.1612730395 C,0,-1.0920169347,-1.2488525237,0.018955904 K,0,3.6695488632,-0.9976573192,-0.8900651068 $\mathbf{H F}=-\mathbf{2 6 1 5 . 5 2 7 6 2 7 2}$

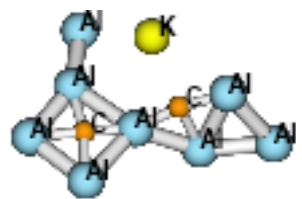

Al,0,2.5475186333,0.8921611782,0.1977389613 Al,0,4.0787913817,-1.2620086475,0.2027886801 Al,0,2.3025169273,3.4791835409,0.6889396013 Al,0,1.9587876352,-3.0045225765,0.2305583157 Al,0,-5.038044902,-0.9734517088,1.0036936762 Al,0,-2.3086176265,-1.2078834721,1.1079797322 Al,0,-3.3220046447,0.248643122,-0.8606261202 Al,0,0.1459126164,-0.8137330661,0.191964566 C,0,-1.5066595058,-0.1530736231,-0.4308074778 C,0,2.058554737,-1.0078271217,0.1876734877 $\mathrm{K}, 0,-0.4239237713,2.1740187189,-1.8137201276$ HF $=-\mathbf{2 6 1 5 . 4 5 9 5 7 9 8}$

o

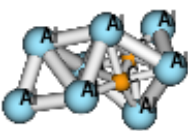

Al,0,-3.4029189743,0.0148976215,1.3569917367 Al,0,-0.6769965373,-1.3156152715,1.1292226915 Al,0,0.134703034,1.5171917307,0.986545007 Al,0,-2.6887175925,1.6344929319,-0.9334215129 $\mathrm{Al}, 0,2.419365708,1.9595861835,-0.2451539856$ Al,0,1.7687870909,-0.675409404,-0.1086245399 K,0,5.3244349635,-0.7975670277,0.0185170246 
Al,0,-0.4827884046,-0.0511377214,-1.4766392948

C, $0,-1.5558931228,0.6262482629,0.5056860337$

C,0,-2.0332142287,-0.549796859,-0.2473874187

Al,0,-3.1967897239,-1.9536164473,-0.855198191

HF $=-\mathbf{2 6 1 5 . 5 0 0 6 1 9 5}$
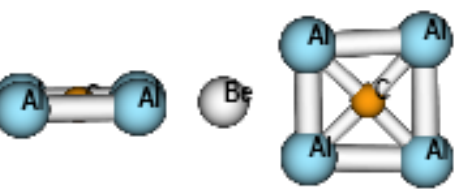

C,0,0.,-2.4516634021,2.4516634021

Al,0,-1.0122468998,-2.7048287174,4.1363410419 Al,0,-0.9471020027,-0.7738012154,2.1131860925 Al,0,0.9471020027,-2.1131860925,0.7738012154 $\mathrm{Al}, 0,1.0122468998,-4.1363410419,2.7048287174$ Be,0,0.,0.,0.

Al,0,0.9470881292,2.1131959025,-0.7737914053 Al,0,-0.9470881292,0.7737914053,-2.1131959025 Al,0,1.0122320721,4.1363515267,-2.7048182325 Al,0,-1.0122320721,2.7048182325,-4.1363515267 C,0,0.,2.4516634021,-2.4516634021 HF $=-\mathbf{2 0 3 0 . 4 6 8 8 2 1 6}$

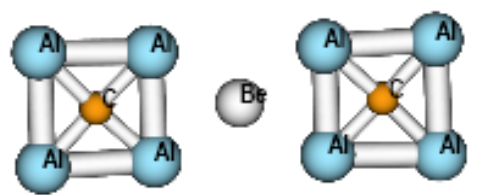

C,0,0.,-2.5231380631,2.5231380631 $\mathrm{Al}, 0,0 .,-2.4675185006,4.5072583401$ $\mathrm{Al}, 0,0 .,-0.5633461571,2.4874706268$ Al,0,0.,-2.4874706268,0.5633461571 $\mathrm{Al}, 0,0 .,-4.5072583401,2.4675185006$ Be,0,0.,0.,0.

Al,0,0.,2.4874706268,-0.5633461571 $\mathrm{Al}, 0,0 ., 0.5633461571,-2.4874706268$ $\mathrm{Al}, 0,0 ., 4.5072583401,-2.4675185006$ $\mathrm{Al}, 0,0 ., 2.4675185006,-4.5072583401$ C,0,0.,2.5231380631,-2.5231380631 HF $=-\mathbf{2 0 3 0 . 4 4 6 9 3 5 1}$

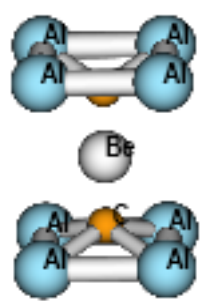

Be,0,0.,0.,0. 
Al,0,1.9751293103,0.000015558,-2.2851878118

Al,0,0.000015558,-1.9751293103,-2.2851878118

Al,0,-1.9751293103,-0.000015558,-2.2851878118

Al,0,-0.000015558,1.9751293103,-2.2851878118

Al,0,-0.000015558,1.9751293103,2.2851878118

Al,0,-1.9751293103,-0.000015558,2.2851878118

Al,0,0.000015558,-1.9751293103,2.2851878118

Al,0,1.9751293103,0.000015558,2.2851878118

C,0,0.,0.,1.6605829843

C,0,0.,0.,-1.6605829843

\section{HF $=-\mathbf{2 0 3 0 . 4 5 4 4 7 8}$}

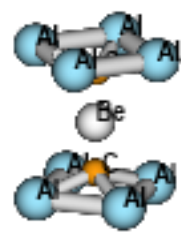

Al,0,0.861343106,1.8274646953,2.2274851548 Al,0,-1.736979252,0.7905239245,2.3250942094 Al,0,-0.7006112434,-1.8097101029,2.297150034 Al,0,1.8979666055,-0.7728791056,2.2000668844 Al,0,1.7385240103,0.7690510979,-2.3307743025 Al,0,-1.8993727487,-0.7867554049,-2.1942499659 Al,0,0.697478459,-1.8292575062,-2.2808206716 Al,0,-0.8580258814,1.811218486,-2.2438356656 C, $0,0.0588569178,0.006177851,1.6563490585$ C,0,-0.0593809807,-0.0056911254,-1.6565433852 Be,0,-0.0002638352,0.0003876381,-0.00008446 HF $=-\mathbf{2 0 3 0 . 4 5 5 8 0 8 3}$

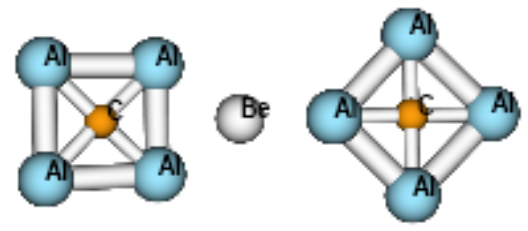

C,0,0.,-2.7177628982,2.7177628982 Al,0,0.,-2.6768417194,4.7074252266 Al,0,0.,-0.764763199,2.6928183663 Al,0,0.,-2.6928183663,0.764763199 Al,0,0.,-4.7074252266,2.6768417194 Be,0,0.,-0.2694719017,0.2694719017 C,0,0.,2.7111727675,-2.7111727675 Al,0,0.,1.3680894156,-1.3680894156 Al,0,0.,4.1192686404,-4.1192686404 Al,0,0.,4.1336535657,-1.3067929196 Al,0,0.,1.3067929196,-4.1336535657 


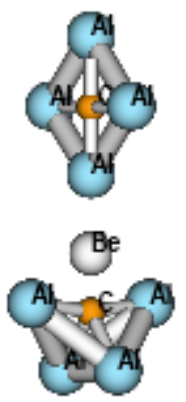

C,0,-2.5579185509,0.,0.

Al,0,-4.1944547063,0.,1.3248884603

Al, $,-2.3202448723,2.0241088446,0$.

Al,0,-4.1944547063,0.,-1.3248884603

Al,0,-2.3202448723,-2.0241088446,0.

Be,0,-0.9079611415,0.,0.

Al,0,1.3197734221,0.,0.

$\mathrm{Al}, 0,5.2080443282,0 ., 0$.

C,0,3.2227631513,0.,0.

Al,0,3.2370513559,0.,1.995163329

$\mathrm{Al}, 0,3.2370513559,0 .,-1.995163329$

HF $=-\mathbf{2 0 3 0 . 4 3 3 3 9 2 6}$
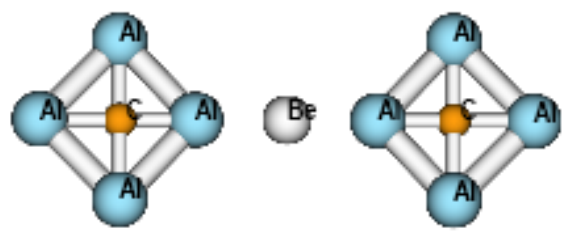

Be,0,0.,0.,0.

Al,0,1.4156598318,3.9477031089,-1.9456284392 $\mathrm{Al}, 0,-0.0000001501,1.6058124839,-1.6058126961$ Al,0,-0.0000004068,4.3521450255,-4.3521456006 Al,0,-1.4156603827,1.9456277852,-3.9477032337 C,0,-0.0000002752,2.9441618885,-2.9441622775 Al,0,0.0000001501,-1.6058124839,1.6058126961 Al,0,0.0000004068,-4.3521450255,4.3521456006 C,0,0.0000002752,-2.9441618885,2.9441622775 Al,0,1.4156603827,-1.9456277852,3.9477032337 Al,0,-1.4156598318,-3.9477031089,1.9456284392 HF $=-\mathbf{2 0 3 0 . 4 2 9 1 8 3 5}$ 


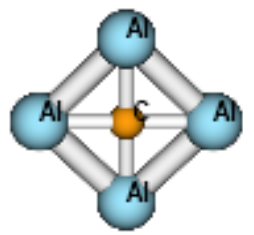

(B)

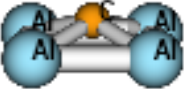

C,0,-2.6081321143,0.,0.

Al,0,-3.2016002407,0.7501747648,1.8389168898 Al,0,-3.2016002407,1.830764263,-0.7698569468 Al,0,-3.2016002407,-0.7501747648,-1.8389168898 Al,0,-3.2016002407,-1.830764263,0.7698569468 Be,0,-0.9779816855,0.,0.

Al,0,1.2877524175,0.,0.

Al,0,5.1729688428,0.,0.

C,0,3.1827432618,0.,0.

Al,0,3.1906959996,-0.7656164284,1.8483615755 Al,0,3.1906959996,0.7656164284,-1.8483615755

HF $=-\mathbf{2 0 3 0 . 4 4 8 7 2 5 9}$

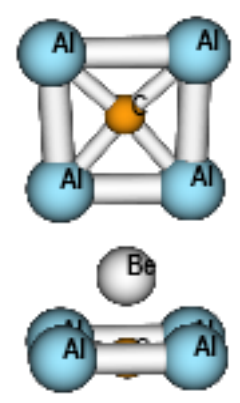

C,0,-2.5302597884,0.,0.

Al,0,-2.3390305557,0.9649070444,1.8329360906 Al,0,-2.3390305557,1.9783738552,-0.6137892194 Al,0,-2.3390305557,-0.9649070444,-1.8329360906 Al,0,-2.3390305557,-1.9783738552,0.6137892194 Be,0,-0.8263824454,0.,0.

Al,0,0.9933120963,0.5015388918,-1.2108219899 Al,0,3.8140451096,-0.5565783643,1.3436990303 C,0,2.5208983426,0.,0.

Al,0,0.9933120963,-0.5015388918,1.2108219899 $\mathrm{Al}, 0,3.8140451096,0.5565783643,-1.3436990303$ HF $=-\mathbf{2 0 3 0 . 4 2 0 9 3 5 2}$ 
C,0,-2.2492975116,0.,0.

Al,0,-3.792608375,0.,1.3779868515

Al,0,-1.928978519,2.0210055933,0.

Al,0,-3.792608375,0.,-1.3779868515

Al,0,-1.928978519,-2.0210055933,0.

Be,0,-0.5628296356,0.,0.

Al,0,1.4399916409,0.,-1.3543082667

$\mathrm{Al}, 0,4.2257884868,0 ., 1.4291226245$

C,0,2.8663465895,0.,0.

$\mathrm{Al}, 0,1.4399916409,0 ., 1.3543082667$

$\mathrm{Al}, 0,4.2257884868,0 .,-1.4291226245$

HF $=-\mathbf{2 0 3 0 . 4 4 5 7 2 4 2}$
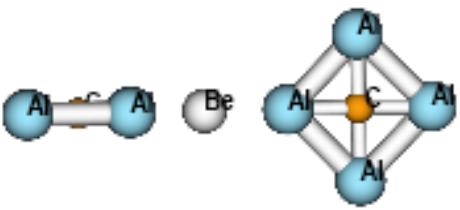

C,0,0.0000002521,-2.6923996268,2.6923999834 Al,0,-1.011827214,-2.9512792155,4.3821989887 Al,0,-0.9535336325,-1.0196760634,2.3681569483 Al,0,0.9535339497,-2.3681567239,1.0196762878 Al,0,1.0118279007,-4.382198503,2.9512797013 Be,0,0.0000000249,-0.2658750921,0.2658751273 Al,0,1.4129356222,3.6900679883,-1.6918459896 Al,0,-0.0000001251,1.3360800964,-1.3360802733 Al,0,-0.0000003829,4.0891771419,-4.0891776835 Al,0,-1.4129361261,1.6918453685,-3.6900680801 C,0,-0.0000002513,2.6836194952,-2.6836198507 HF $=-\mathbf{2 0 3 0 . 4 5 3 8 0 6 7}$

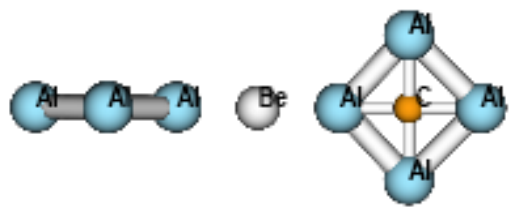

Be,0,0.,0.,0.

Al,0,1.4147898088,3.9311355555,-1.9302912987 Al,0,-0.000000149,1.5870643962,-1.5870646058 
Al,0,-0.0000004069,4.3347860897,-4.3347866621

Al,0,-1.4147903591,1.9302906468,-3.9311356775

C,0,-0.0000002749,2.9285326232,-2.9285330098

$\mathrm{Al}, 0,0.000000149,-1.5870643962,1.5870646058$

Al,0,0.0000004069,-4.3347860897,4.3347866621

C,0,0.0000002749,-2.9285326232,2.9285330098

Al,0,-1.4148105406,-1.9303054392,3.9311211508

Al,0,1.4148110908,-3.9311207631,1.9303058254

$\mathbf{H F}=-\mathbf{2 0 3 0 . 4 3 5 5 8 9 7}$
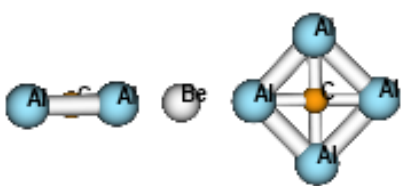

C,0,-3.8079787367,-0.0077458937,0.0013487199 $\mathrm{Al}, 0,-5.1854828089,-0.5516529877,1.3260222484$ Al,0,-2.3943146389,-0.5180053924,1.2479305565 Al,0,-2.3969151201,0.5075971608,-1.2462264011 Al,0,-5.187734346,0.531313321,-1.3232075609 Be,0,-0.3759743606,-0.0016065858,0.0002688789 Al,0,3.811544016,-1.8377032701,-0.7667003585 Al,0,1.8894535641,0.002638566,-0.0003586658 $\mathrm{Al}, 0,5.7837067206,0.0136169458,-0.0017753177$ Al,0,3.8014416036,1.8528389498,0.7642322166 C,0,3.7949471642,0.007423149,-0.0013475274 HF $=-\mathbf{2 0 3 0 . 4 5 3 8 1 2 6}$

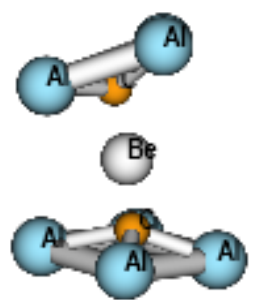

Al,0,2.039860558,-1.5524075382,1.2972535445 Al,0,1.795947865,1.3271532618,1.5226689822 Al,0,2.6000366913,-1.290683202,-1.4879049742 Al,0,2.4398264181,1.5301078648,-1.2526049936 Al,0,-2.6067059935,0.0488641305,-1.9604436484 Al,0,-2.4421710654,-1.9744736967,0.0185883366 Al,0,-1.7891283023,-0.0495950888,2.0251694423 $\mathrm{Al}, 0,-2.0373042833,2.0255346002,0.0164344753$ C,0,1.6611193644,-0.0401064871,-0.1260556052 C,0,-1.6616233569,-0.0474402754,-0.1171761652 Be,0,-0.0004201464,-0.0783059341,-0.2174261298 HF $=-2030.4544773$ 


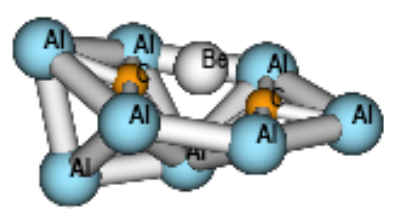

Al,0,2.1494405828,-1.0818540909,1.5764957967 Al,0,1.1099443953,2.062700049,0.6878412469 Al,0,1.2578733215,-1.6434568434,-0.9982359537 Al,0,3.2434782268,0.5730273395,-0.5251648342 Al,0,-3.7303166272,-0.0250955612,-0.5381910386 Al,0,-1.5724578346,-1.7803428822,-1.1857758738 Al,0,-0.315955713,-0.3403517736,1.2025438195 Al,0,-1.8480970788,1.9485002029,0.4180860609 C,0,1.2509805571,0.2816805459,-0.2713071907 C,0,-1.7460741927,0.1051621917,-0.4204704588 Be,0,-0.212564683,0.3520749634,-1.0345310031 HF $=\mathbf{- 2 0 3 0 . 4 8 8 9 5 0 7}$

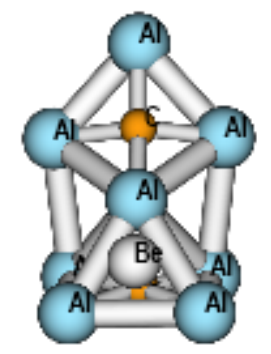

Al,0,2.5068074288,1.4604765525,0.7937236666 Al,0,1.0454260397,1.5268050036,-1.4443905818 Al,0,1.0116381099,-1.5029725781,-1.4928330083 Al,0,2.4733733174,-1.5412811976,0.7453262395 Al,0,-3.8214416999,0.0399247726,0.2244719109 Al,0,-0.0278877236,-0.0165507254,1.0102904797 Al,0,-1.7813688032,1.9079784667,-0.2631224313 Al,0,-1.8242649893,-1.8573819971,-0.3269341576 C,0,-1.8649770988,0.0152927672,0.3405957047 C,0,1.5982529994,-0.0131080436,-0.3000013407 Be,0,1.7576706895,-0.0585215509,2.387879072 HF $=-2030.4281811$

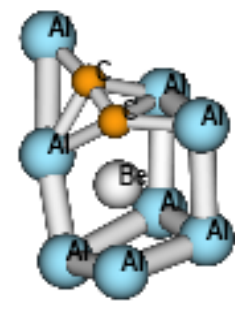

Al,0,-1.3803508136,1.2985976984,-0.2465244848 Al,0,-2.0943711542,-0.6320977288,-1.7638373218 
Al,0,-1.918129032,-0.4928216685,1.8597048642 Al,0,-2.2632185279,-2.4756374595,-0.0014994953 Al,0,1.3679370697,1.7451987977,-0.9168467928 Al,0,0.6788234471,-0.5280189317,-2.3102119308 Al,0,0.6976224655,0.1437688346,1.8425183527 Al,0,3.3933592309,1.3571000722,1.4572025996 Be,0,-0.2674545044,-1.1106471805,-0.0691985502 C,0,1.2237033622,-0.4166377112,-0.1934178771 C,0,2.2443088224,0.255541667,0.4117876966 HF $=-2030.4564891$

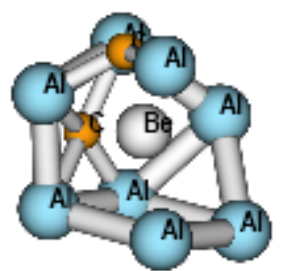

Al,0,-1.7113192007,1.8426924279,-1.7669155125 Al,0,-1.8286237549,-0.8781932191,-1.4742036167 Al,0,-1.4759794263,1.227629073,0.8420118152 Al,0,-1.6107141508,-1.4180252885,1.1370537142 Al,0,0.6055284182,2.3987927013,-0.5279901513 Al,0,2.1034648991,-1.8227735662,-1.5577246703 Al,0,1.9490254564,0.8005994659,1.2242182619 C, $0,-0.0087341419,0.1006074246,1.5050235137$ Be,0,0.3333111177,-0.2369989526,-0.2671918019 Al,0,0.9110952714,-1.7246710138,1.4729167019 C,0,2.0778254531,-0.8657177138,0.0828101793 $\mathbf{H F}=\mathbf{- 2 0 3 0 . 4 1 9 2 9 6 2}$

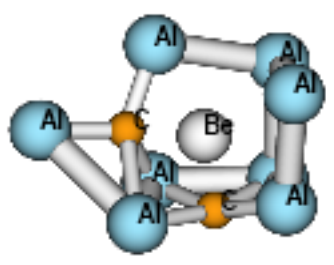

Al,0,-1.835433695,1.7165770152,-0.6442740216 Al,0,-2.5763907299,-0.6483471125,-1.4577835577 Al,0,-1.2006942467,0.9468689408,2.0065070797 Al,0,-1.852397102,-1.4627405814,0.9861435782 Al,0,0.6419592588,1.3059942683,-1.9444939913 Al,0,3.2748117053,-0.4062478306,-1.2959989697 Al,0,1.4014563904,-1.8833650143,0.5924973542 Al,0,1.5181441226,0.7612539612,1.4653225644 Be,0,-0.1571983086,-0.0517368702,-0.2168931133 C,0,1.5207663795,0.035936833,-0.6370905115 C,0,-0.0541215314,-0.7164318206,1.4145258421 $H F=-2030.4438272$ 


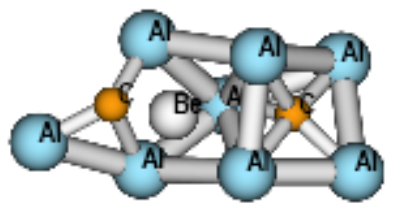

Al,0,-0.3466780431,-0.6496654148,-1.3686313287 Al,0,0.2334310151,1.719195365,0.1050237832 Al,0,2.1695805791,-0.1870979644,-0.5316629489 Al,0,3.8188239482,-1.4935833456,1.299298838 Al,0,0.2817179919,-2.1740139956,0.9457443012 Al,0,-1.8848277968,2.7657300353,-1.2155046678 Al,0,-1.5792495229,-0.0396748464,1.2449376804 Al,0,-3.1678780089,0.1525832411,-1.3579373948 Be,0,0.8890058138,0.1070831325,1.6676933142 C,0,-1.4879834786,0.8895785504,-0.5788598244 C,0,1.9246525837,-1.1634923004,1.3709830458 HF $=-2030.472462$

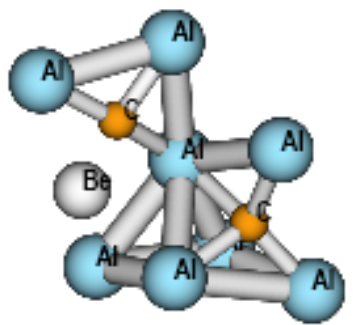

Al,0,1.0389719,0.6734076833,1.568074009 Al,0,1.4378041626,-1.9663671931,-0.7070118017 Al,0,-0.781958834,-0.2449709399,-0.3927551599 $\mathrm{Al}, 0,0.7769838546,-2.1620073402,2.603134363$ Al,0,-0.049730794,-0.3900856476,-3.101354381 Al,0,-1.2492974771,1.8703391428,1.5712829186 Al,0,-1.6561022392,-0.8635248531,1.9992341077 Be,0,-0.0695665046,2.0563395112,-0.4378994275 Al,0,0.221150491,2.4970450195,-3.0149576983 C,0,-0.0320527759,1.0567040456,-1.7438631191 C,0,0.6464848069,-1.1575747748,0.8968956299 HF $=-\mathbf{2 0 3 0 . 4 4 9 2 1 2 1}$

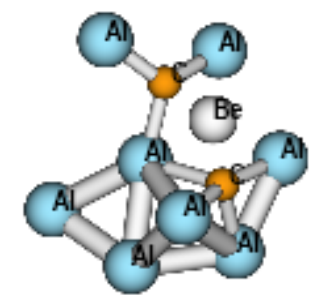

Al,0,0.3182286356,0.4575105352,-0.494497898 Al,0,-0.0487408772,2.3973334714,-2.3365390336 Al,0,3.3951328984,1.0532572218,0.5809416295 Al,0,2.4596054867,-2.1361644056,0.1858711188 
Al,0,-0.6119704278,-2.6312754829,0.5895656196 Al,0,-2.0860369343,1.3970658865,-1.1589188894 Al,0,-1.5940148029,1.1263338659,1.7274115693 Al,0,-2.5149509919,-1.0568103962,-0.2121431666 C,0,-0.9425305512,-0.6187032159,0.9930923342 C,0,1.9781567448,-0.2209574795,0.4861998038 Be,0,0.6654885032,-0.7140737194,1.4155662067

\section{HF $=-\mathbf{2 0 3 0 . 4 6 0 2 4 9 3}$}

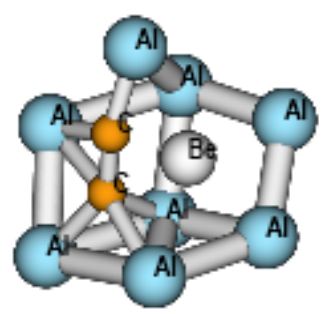

Al,0,-1.7186740981,1.7693191537,-0.5942532929 Al,0,0.6837901369,2.5331673241,1.4577009913 Al,0,1.1199499337,1.5053032301,-1.2857651312 Al,0,2.9210018616,-0.078885686,-0.2975026615 Al,0,-2.8923184628,-0.7933400622,-0.3265044341 $\mathrm{Al}, 0,-0.8845476041,-2.1329403782,1.2657461544$ Al,0,1.5840723242,-2.2570011632,0.1129486435 Al,0,-0.2813391331,-0.9810232224,-1.3391648954 Be,0,0.7596054219,-0.0722939204,0.8338203098 C,0,-0.5287739196,1.0581831026,1.0700547707 C,0,-1.130155438,-0.0666187469,0.5554533788 HF $=-\mathbf{2 0 3 0 . 4 4 9 1 0 7 2}$

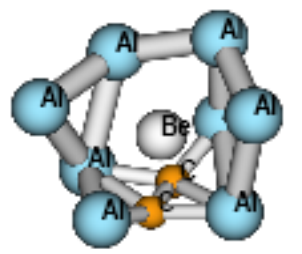

Al,0,1.8836704585,-1.9598849204,-0.4931320816 $\mathrm{Al}, 0,2.388664645,0.4696225828,-1.5854202749$ Al,0,1.4269884687,-0.5843223006,1.664540628 Al,0,1.3750294581,2.1372535685,0.3929851435 Al,0,-0.6408402028,-2.3225149139,0.0321406633 Al,0,-2.5847365371,-0.9582217382,-1.1485914639 Al,0,-1.6812181244,2.0817482557,-0.9652468799 Al,0,-1.9159907175,0.0364874323,1.2423251171 C, $0,0.0108127625,0.7022847538,1.4617799529$ Be,0,0.0609333343,0.0698041932,-0.3136852989 C,0,-0.5964977901,1.6341485237,0.6115417344 HF $=-2030.4381586$ 


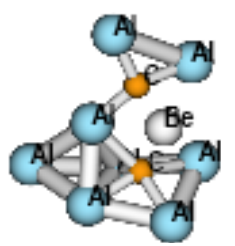

Al,0,-0.3090330755,1.2992080978,-0.3025267119 Al,0,-3.4900837951,1.5948549185,-0.4084166883 Al,0,-3.0129301393,-1.1743782984,0.5786022472 Al,0,0.2887250557,-2.4611350562,0.8210349269 Al,0,1.8311503054,-2.028531955,-1.408465862 $\mathrm{Al}, 0,1.9348628841,2.6271364706,0.7698259564$ $\mathrm{Al}, 0,2.144019786,0.6701523294,-1.107073559$ Al,0,1.4756544221,-0.1130760411,1.5301242779 Be,0,-0.9084193347,-0.8930281904,-0.4517837117 C,0,-2.0447208467,0.3477993062,-0.3772335764 C,0,0.7818752762,-0.6499465214,-0.3466372215 HF $=-\mathbf{2 0 3 0 . 4 7 7 9 8 0 4}$

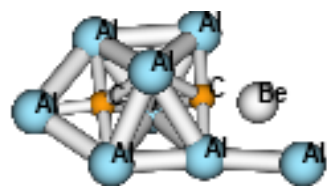

Al,0,-0.7401862044,-1.7708959621,-2.1744838125 Al,0,-1.3961647317,0.2862171672,-0.4506220458 Al,0,-0.4686231241,-1.3468461919,1.9170346357 Al,0,-1.3171656733,-3.4464963451,0.2007036753 Al,0,1.555185408,3.9795564987,1.9289034427 Al,0,1.211980329,-0.7233385816,-0.520900919 Al,0,0.3704724776,1.1045454692,-2.405353287 Al,0,0.52804108,1.2522222329,1.6990546947 C,0,0.4628894016,1.1935855272,-0.306332994 C,0,-0.6048007048,-1.5777121766,-0.0499328839 Be,0,1.0463633814,2.7375560408,-0.0971944318 HF $=-\mathbf{2 0 3 0 . 4 3 4 0 8 0 6}$

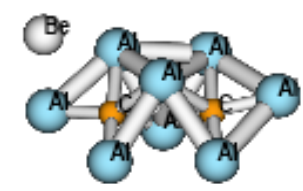

Al,0,-0.0832759192,-1.1222057248,-2.1824501832 Al,0,-1.4327545017,0.1184975309,-0.2687353429 Al,0,-0.4172666171,-2.0772422035,1.8216515956 Al,0,-0.1573127563,-3.518394524,-0.687331506 Al,0,-0.1784094519,3.3735312423,0.360195109 $\mathrm{Al}, 0,1.2903762121,-0.2908750994,0.186632041$ 
Al,0,0.5399255699,1.519013849,-1.5997400216

Al,0,-0.0042123206,1.0481935293,2.4530907498

C,0,0.0180020313,1.3490397911,0.5120293913

C,0,-0.3336351655,-1.5961217415,-0.0939939916

Be,0,1.9129715017,3.4564374765,-0.8978185349

HF $=-\mathbf{2 0 3 0 . 4 0 1 8 2 3 8}$

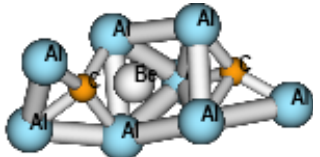

Al,0,3.3755498209,-1.3810054865,0.4178759155

Al,0,0.6585316233,-1.5895412587,0.4513482076 Al,0,3.2907488169,0.9007509462,-1.2605440196 Al,0,0.9285168266,1.2834934536,1.0570916226 Al,0,-1.3408778864,-0.127578596,1.6863400817 Al,0,-1.2845350711,1.8727751039,-0.4716791663 Al,0,-1.4098255614,-0.8147263709,-1.1406181914 Al,0,-4.1392433969,-0.4101883836,-0.4123548797 C,0,1.9528382097,-0.0491654717,-0.1393449058 C,0,-2.5333324058,0.4250571041,0.1992662087 Be,0,0.6144294852,0.3007294753,-1.1541255582 HF $=-\mathbf{2 0 3 0 . 4 6 8 0 0 8 3}$
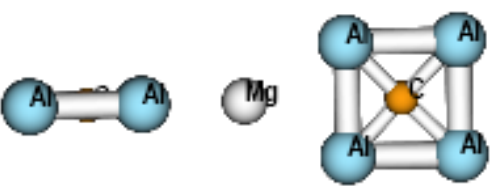

C,0,0.,-2.7484726475,2.7484726475

Al,0,-0.9973585101,-3.0381838468,4.4486411171 Al,0,-0.9928241876,-1.0872385059,2.4912833698 Al,0,0.9928241876,-2.4912833698,1.0872385059 $\mathrm{Al}, 0,0.9973585101,-4.4486411171,3.0381838468$ Mg,0,0.,0.,0.

Al,0,0.9928096444,2.4912936535,-1.0872282223 Al,0,-0.9928096444,1.0872282223,-2.4912936535 Al,0,0.9973439004,4.4486514478,-3.0381735162 Al,0,-0.9973439004,3.0381735162,-4.4486514478 C,0,0.,2.7484726475,-2.7484726475 $\mathbf{H F}=\mathbf{- 2 2 1 5 . 8 3 1 8 0 8 7}$
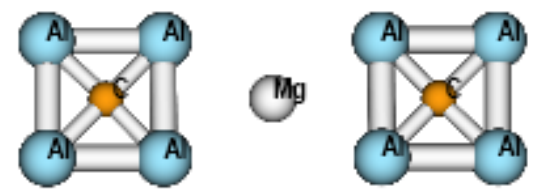

C,0,0.,-2.7887003527,2.7887003527 $\mathrm{Al}, 0,0 .,-2.7828159861,4.7815813278$ $\mathrm{Al}, 0,0 .,-0.8349196692,2.8267499151$ 
$\mathrm{Al}, 0,0 .,-2.8267499151,0.8349196692$

Al,0,0.,-4.7815813278,2.7828159861

Mg,0,0.,0.,0.

Al,0,0.,2.8267499151,-0.8349196692

Al,0,0.,0.8349196692,-2.8267499151

$\mathrm{Al}, 0,0 ., 4.7815813278,-2.7828159861$

$\mathrm{Al}, 0,0 ., 2.7828159861,-4.7815813278$

C,0,0.,2.7887003527,-2.7887003527

HF $=-\mathbf{2 2 1 5 . 8 2 3 5 6 6 2}$

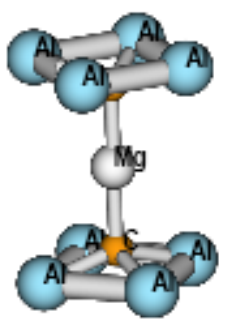

Al,0,0.0002764257,1.9808028091,2.6665837598

Al,0,-1.9808031933,0.0002950354,2.6665795589 Al,0,-0.0002936699,-1.980786096,2.6665738526 Al,0,1.9807865308,-0.0002754003,2.6665906315 Al,0,1.4002667843,1.4006064988,-2.6667972578 $\mathrm{Al}, 0,-1.4002514316,-1.4006252658,-2.666785074$ Al,0,1.4006660788,-1.4002342565,-2.6667671469 $\mathrm{Al}, 0,-1.4006517774,1.400217741,-2.6667697054$ C,0,-0.0000073029,0.0000106351,2.1035272756 C,0,0.0000075268,-0.0000064968,-2.1026426338 Mg,0,0.0000044951,-0.0000032236,0.0004150089 $\mathrm{HF}=-\mathbf{2 2 1 5 . 7 8 2 8 9 2 7}$

Al,0,1.3890707125,1.4081696488,2.6757917058 Al,0,-1.4080985783,1.389432172,2.6763511291 Al,0,-1.3894732065,-1.407758975,2.6756795694 Al,0,1.4078159313,-1.3891201914,2.6751397758 Al,0,1.4084550754,1.3888210691,-2.676008809 Al,0,-1.4080571692,-1.3892345314,-2.6761138924 Al,0,1.3898323523,-1.4078631744,-2.6760447037 Al,0,-1.3895594423,1.4075496974,-2.6748176006 C, $0,-0.0002943771,0.0003156525,2.102760444$ C,0,0.0002830542,-0.0003042177,-2.1027780482 Mg,0,0.0000211801,-0.0000010753,0.0000335297 $\mathrm{HF}=-\mathbf{2 2 1 5 . 7 8 2 6 0 0 1}$ 

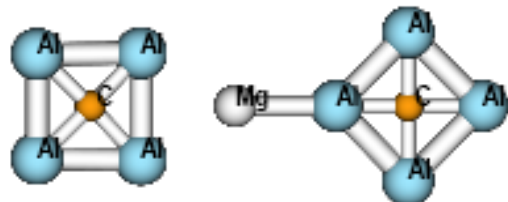

C,0,0.,-2.9603022344,2.9603022344

Al,0,0.,-2.9665114684,4.9581855146

Al,0,0.,-1.0117194349,3.0199508317

Al,0,0.,-3.0199508317,1.0117194349

Al,0,0.,-4.9581855146,2.9665114684

Mg,0,0.,-0.2380124677,0.2380124677

C,0,0.,3.0229424357,-3.0229424357

Al,0,0.,1.6767824891,-1.6767824891

Al,0,0.,4.4290758896,-4.4290758896

Al,0,0.,4.4336736984,-1.6076281267

Al,0,0.,1.6076281267,-4.4336736984

HF $=-2215.812971$

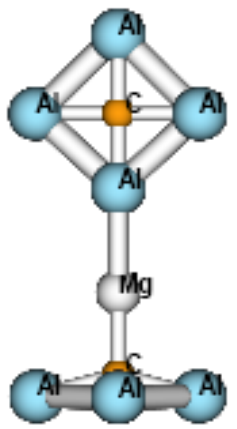

C,0,-3.0028075347,0.,0.

Al,0,-3.5308704631,0.,1.9888762821

Al,0,-3.5440881408,1.9824048765,0.

Al,0,-3.5308704631,0.,-1.9888762821

Al,0,-3.5440881408,-1.9824048765,0.

Mg,0,-0.8954952733,0.,0.

Al,0,1.7513708844,0.,0.

Al,0,5.637740672,0.,0.

C,0,3.647843441,0.,0.

Al,0,3.6448538966,0.,2.0011020603

Al,0,3.6448538966,0.,-2.0011020603

$H F=-2215.7914029$

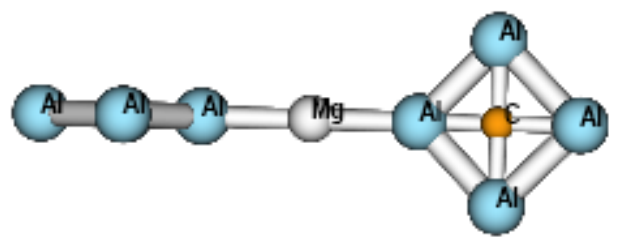

Mg,0,0.,0.,0.

Al,0,1.4150250156,4.2096074999,-2.2084306054

Al,0,-0.0000001757,1.8714932082,-1.8714934553 
Al,0,-0.0000004337,4.6193729104,-4.6193735203

Al,0,-1.4150256181,2.2084299167,-4.2096076586 C,0,-0.0000003016,3.2130843294,-3.2130847537 Al,0,0.0000001757,-1.8714932082,1.8714934553 Al,0,0.0000004337,-4.6193729104,4.6193735203 C,0,0.0000003016,-3.2130843294,3.2130847537 Al,0,-1.4150457508,-2.2084447115,4.2095931295 Al,0,1.4150463533,-4.2095927051,2.2084451345 HF $=-\mathbf{2 2 1 5 . 7 9 8 7 1 1 2}$

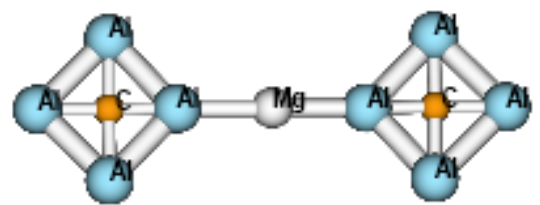

Mg,0,0.,0.,0.

Al,0,1.4152445803,4.2183261676,-2.2168387608 Al,0,-0.0000001759,1.8817640773,-1.8817643259 Al,0,-0.0000004327,4.6291575647,-4.6291581764 $\mathrm{Al}, 0,-1.4152451818,2.2168380711,-4.2183263283$ C,0,-0.0000003012,3.2225126326,-3.2225130584 Al,0,0.0000001759,-1.8817640773,1.8817643259 Al,0,0.0000004327,-4.6291575647,4.6291581764 C,0,0.0000003012,-3.2225126326,3.2225130584 Al,0,1.4152451818,-2.2168380711,4.2183263283 $\mathrm{Al}, 0,-1.4152445803,-4.2183261676,2.2168387608$ HF $=-2215.7959813$

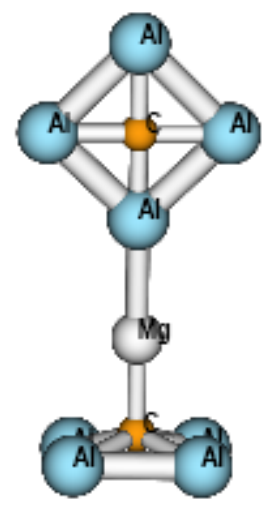

C,0,-3.0042266494,0.,0.

Al,0,-3.536490335,0.7555937038,1.8365739591 Al,0,-3.536490335,1.8329393294,-0.7643684761 Al,0,-3.536490335,-0.7555937038,-1.8365739591 Al,0,-3.536490335,-1.8329393294,0.7643684761 Mg,0,-0.8960312829,0.,0.

$\mathrm{Al}, 0,1.7510582474,0.0$.

$\mathrm{Al}, 0,5.6370175507,0 ., 0$.

C,0,3.6477680311,0.,0. 
Al,0,3.6439861211,-0.7658074187,1.8488226668

Al,0,3.6439861211,0.7658074187,-1.8488226668

HF $=-2215.791422$
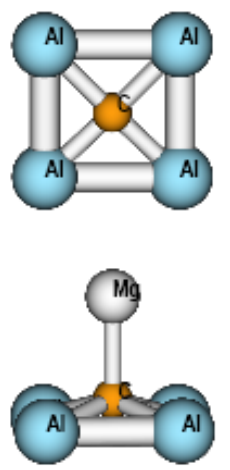

C,0,-2.7102601959,0.,0.

Al,0,-3.2339067098,0.7518580682,1.8334277192

Al,0,-3.2339067098,1.8280731137,-0.7647852295

Al,0,-3.2339067098,-0.7518580682,-1.8334277192

Al,0,-3.2339067098,-1.8280731137,0.7647852295

Mg,0,-0.5672602837,0.,0.

Al,0,1.9228352166,0.5405491277,-1.3050010301

Al,0,4.678315022,-0.5398575577,1.3033314326

C,0,3.2669878805,0.,0.

Al,0,1.9228352166,-0.5405491277,1.3050010301

Al,0,4.678315022,0.5398575577,-1.3033314326

$\mathbf{H F}=-\mathbf{2 2 1 5 . 8 0 8 5 1 2 6}$
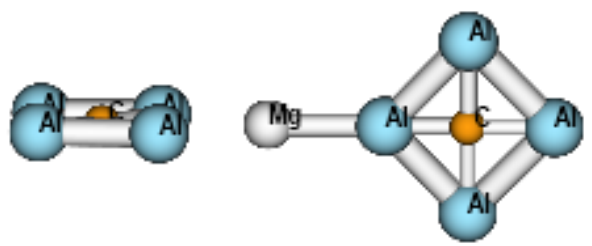

C,0,0.0000002758,-2.9455350602,2.9455354503 Al,0,-0.994885544,-3.2437445453,4.6507056043 Al,0,-1.001310955,-1.2905888032,2.7066361162 Al,0,1.0013113293,-2.7066358515,1.2905890679 Al,0,0.9948862833,-4.6507050815,3.2437450682 Mg,0,0.0000000223,-0.2376990747,0.2376991062 Al,0,1.4130730808,4.0050135609,-2.0065971635 Al,0,-0.0000001554,1.6594299244,-1.6594301442 Al,0,-0.0000004131,4.4119032991,-4.4119038835 $\mathrm{Al}, 0,-1.4130736437,2.0065965007,-4.0050136944$ C,0,-0.0000002815,3.0065170351,-3.0065174333 $\mathbf{H F}=-\mathbf{2 2 1 5 . 8 1 7 1 6 0 9}$ 

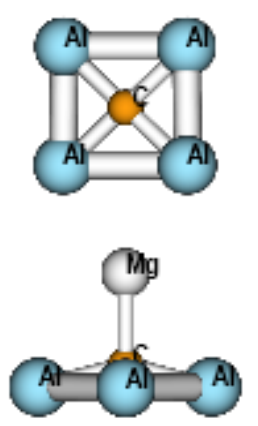

C,0,-2.7088775573,0.,0.

Al,0,-3.2242889041,0.,1.986967925

Al,0,-3.2388950485,1.9770990015,0.

Al,0,-3.2242889041,0.,-1.986967925

Al,0,-3.2388950485,-1.9770990015,0.

Mg,0,-0.5670353553,0.,0.

Al,0,1.9205707476,0.,-1.4127513778

Al,0,4.6760455331,0.,1.4107471366

C,0,3.2647415127,0.,0.

Al,0,1.9205707476,0.,1.4127513778

Al,0,4.6760455331,0.,-1.4107471366

$\mathbf{H F}=-2215.8087117$

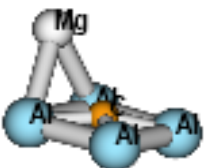

C,0,-3.035058985,-0.0633074695,-0.2685119517 Al,0,-2.8667670524,0.6049450414,1.6144755286 Al,0,-2.6434257295,1.6882436971,-1.1625877994 Al,0,-3.8142801814,-0.6271614293,-2.0388564911 Al,0,-4.0205945179,-1.6290741266,0.5290876309 Mg,0,-0.410909513,0.6977425374,0.239363483 Al,0,1.9320932508,-0.4134211129,1.5183658611 Al,0,4.8548261809,0.1183304723,-1.0795591981 C,0,3.358586867,-0.1465233485,0.212665502 Al,0,4.6449857497,-0.901223681,1.5371755595 Al,0,2.1431428279,0.6121360973,-1.1132767145 $\mathbf{H F}=-2215.8004097$

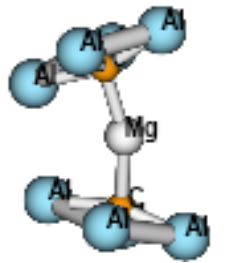


Al,0,-1.8064154843,0.1391405568,3.069901118 Al,0,-0.0850974483,1.9047690446,1.8306015037 Al,0,0.0098081707,-1.9644703903,3.0377946584 Al,0,2.0030473818,-0.1133678408,2.1433985082 Al,0,1.8106244003,0.2805710439,-3.0576737144 Al,0,-0.0471627148,-1.7847718467,-3.1464321404 Al,0,-2.0036735827,0.050788216,-2.1452824325 $\mathrm{Al}, 0,0.1240706461,2.0054330778,-1.7173012302$ C,0,-0.0427159811,-0.1778192687,2.0931213609 C,0,0.0403659799,-0.0572146539,-2.0999281883 Mg,0,-0.0044598156,-0.4437492218,-0.0128533798 HF $=-\mathbf{2 2 1 5 . 7 7 8 3 9 0 9}$

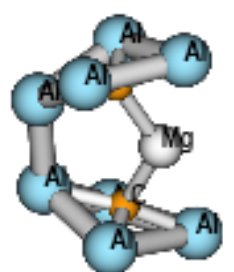

Al,0,-1.6812181472,0.4136214559,3.0492818317 Al,0,-0.0619089607,1.8656589088,1.4613509311 $\mathrm{Al}, 0,-0.2678368845,-1.9505053271,2.781388418$ Al,0,1.9702001673,-0.03212474,1.8451420029 Al,0,1.6910824965,0.5559190733,-3.0208987003 Al,0,0.2305940341,-1.7911371856,-2.8898633274 Al,0,-1.9693818267,0.1137206423,-1.8429155763 Al,0,0.0995855277,1.9455862019,-1.3510432495 C,0,-0.065671216,-0.1521767431,1.9169699204 C,0,0.0637855623,-0.0422458603,-1.9225603222 Mg,0,-0.0110999469,-1.116922647,-0.0323506567 $H F=-2215.7817963$

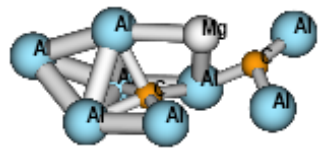

Al,0,0.0327383266,2.3016252367,-0.0183935515 $\mathrm{Al}, 0,2.4361691002,1.2807863762,-0.6444065033$ Al,0,-0.885338801,-0.7570371127,-0.979867239 Al,0,4.0043928895,-0.8468571457,0.2965581054 Al,0,-3.0964696481,1.4377194042,-0.2498384621 Al,0,1.5201411287,-0.4230499958,1.4343541649 Al,0,1.5880754293,-1.2286183624,-1.1866901516 Mg,0,-1.0855858255,0.2004789762,1.66478332 Al,0,-3.6979839092,-1.9457023881,-0.0300821433 C,0,-2.5573779096,-0.428357591,0.0218441805 C, $0,0.6081464424,0.4198566118,-0.3649516294$ HF $=-2215.7910504$ 


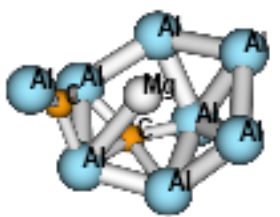

Al,0,-2.0052487535,1.9643693361,-1.6731159891 Al,0,-2.7303652082,-0.7067050151,-1.1066146689 Al,0,-1.5761334316,1.1259316188,0.8633666604 Al,0,-1.5109006335,-1.5196369167,1.1282349866 Al,0,0.3435709593,2.4988847203,-0.4096566318 Al,0,3.3204978605,-1.9068496061,-1.0697872931 $\mathrm{Al}, 0,1.8497974089,0.8748545404,1.0237565198$ C,0,-0.0175710613,0.1364119482,1.4359583977 Mg,0,0.2665981692,-0.4134966271,-1.0097786502 Al,0,0.994484939,-1.6060008883,1.4393130453 C,0,2.3320179177,-0.8799222376,0.1600228725 HF $=-\mathbf{2 2 1 5 . 7 5 8 3 7 4 2}$

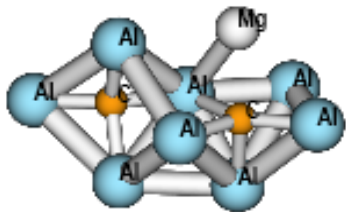

Al,0,3.9785538733,-0.02667753,-0.2401147431 Al,0,1.7023321731,-1.7730278964,0.6227434673 Al,0,1.73708831,1.9012185859,0.134671866 Al,0,-2.2767494844,-0.2622772941,-1.8633713847 Al,0,-1.1550932293,1.9658576064,-0.3424456598 Al,0,-3.3407965026,0.0845023038,0.7127020039 Al,0,-0.0981341095,0.2740365782,1.9756695856 C,0,-1.3614048729,0.0607399881,0.503941027 Mg,0,-1.2106859365,-2.1838443401,0.0563143083 Al,0,0.2551509646,-0.1564147549,-1.1326895719 C,0,2.0443474229,-0.0420227725,-0.3287616975 HF $=-2215.790896$

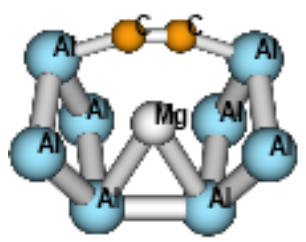

Al,0,-1.7290741239,1.7945515688,-0.2485892036 Al,0,-2.5548500596,-0.478176167,-1.4592905531 Al,0,-1.2685233363,0.6082434755,2.079050376 Al,0,-2.7146276384,-1.400747286,1.0805229586 Al,0,1.5226288297,1.9273097331,-0.4651568772 Al,0,2.3919988193,-0.2740305483,-1.7688392789 Al,0,2.937495713,-1.1527861442,0.7339745009 
Al,0,1.4526248404,0.7338022402,1.9039765281

Mg,0,0.047022882,-0.945285627,0.0896827202

C,0,-0.7229094327,-0.9861083565,-2.0601079475

C,0,0.5472387392,-0.9326819458,-2.1398291364

HF $=-2215.7735724$

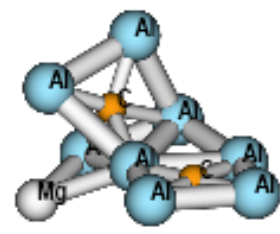

Al,0,2.4838854165,0.1038539174,-1.5302867591 Al,0,0.9852503584,-1.9603610952,-0.8648448282 Al,0,2.1930313407,1.1443776862,1.5344541509 Al,0,1.9241758188,-1.6677818196,1.6911403603 Al,0,-1.8265570097,-1.8058843355,-0.6918538092 Al,0,-4.0058200096,-0.5035696082,0.2136448881 Al,0,-2.5453959907,1.8398111965,0.6478500997 Al,0,-0.2353749216,0.5169677748,-0.2751289259 C,0,1.4788160898,-0.2473848665,0.3566052043 C,0,-2.0949687454,0.0302122526,-0.0034081629 Mg,0,1.4204484082,2.6355547807,-0.9619882953 HF $=-\mathbf{2 2 1 5 . 7 9 8 5 9 5 3}$

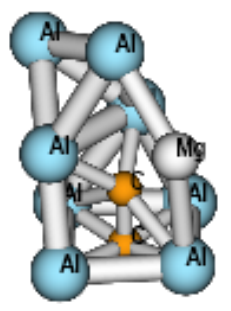

Al,0,-1.7995906604,-0.3659079179,2.0777492837^M Al,0,-1.0139740587,-2.1216151574,-0.0922264777^M $\mathrm{Al}, 0,2.9293596235,1.1326355336,0.0695388542 \wedge \mathrm{M}$ $\mathrm{Al}, 0,0.8949683185,-0.3122224259,-1.4448704459 \wedge \mathrm{M}$ $\mathrm{Al}, 0,3.0246199003,-1.4036561796,-0.0318150228 \wedge \mathrm{M}$ Al,0,-1.765234904,-0.2001061343,-2.1302091221^M $\mathrm{Mg}, 0,0.4008545957,2.3058255693,0.0949437033^{\wedge} \mathrm{M}$ $\mathrm{Al}, 0,-2.3542554672,1.6409189177,0.0459439805^{\wedge} \mathrm{M}$ $\mathrm{C}, 0,-0.5548998635,0.16032535,0.0022753866 \wedge \mathrm{M}$ $\mathrm{Al}, 0,0.8724764861,-0.4265628834,1.430473166 \wedge \mathrm{M}$ $\mathrm{C}, 0,-1.9549426775,-0.3161912862,-0.0287619279 \wedge \mathrm{M}$ HF $=-2215.7884894$ 


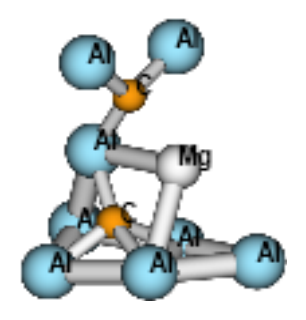

Al,0,2.6415521165,2.0044855857,-1.3109070402 Al,0,2.1280345636,0.8573739571,1.0540157734 Al,0,1.7140800453,-0.6707180598,-1.1906977605 Al,0,0.5668333035,-2.924283494,-0.125829683 Al,0,-3.0632691311,0.6783922166,-1.9092667111 Al,0,-3.3177009467,1.2498611351,1.2540568058 Al,0,1.4086593571,-1.5551337274,2.1477721705 Al,0,-1.170555152,-1.0766062241,0.0872995708 Mg,0,-0.1978434248,1.5246189871,-0.216210917 C,0,-2.2489278463,0.5838333586,-0.1772412905 C,0,0.6780740241,-0.5203760092,0.5957030188 $\mathbf{H F}=-2215.7935488$

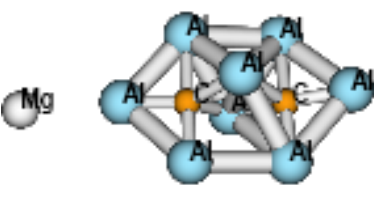

Al,0,-0.6552373126,-1.8341508022,-2.14249991 Al,0,-1.4738860311,-0.1797825564,-0.2101764684 Al,0,-0.634394106,-2.1202053256,2.0041809485 Al,0,-0.7042738077,-4.0021915804,-0.2536475556 Al,0,0.3043427418,2.7943994462,-0.0003542493 Al,0,1.1939019638,-0.9781443742,-0.2124650901 Al,0,0.3894537153,0.8219863336,-2.0025178367 Al,0,0.2752777383,0.7824300565,2.1683745356 C,0,0.3029931918,0.8222959666,0.1974660763 C,0,-0.5881512463,-2.0121352771,0.0317030817 Mg,0,1.5561287169,5.7035500246,0.5886131825 HF $=-2215.7925293$

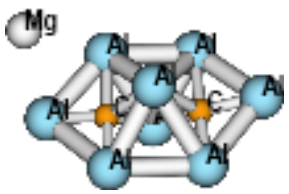

Al,0,-0.6065505802,-1.3242552575,-2.1089066268 Al,0,-1.5596270682,0.2753571503,-0.1938798332 Al,0,-1.2455310964,-1.9630374946,1.9403137011 Al,0,-1.2600685216,-3.5906731395,-0.4936808542 Al,0,0.4701294221,3.048730426,0.514812744 Al,0,0.9654985192,-0.8312612919,0.1705627317 
Al,0,0.6487820919,1.1501690091,-1.5835868026

Al,0,-0.0064216693,0.8126374926,2.4869386204

C,0,0.2072667108,1.0515726055,0.5426230317

C,0,-0.9400667098,-1.6627782929,0.009357753

Mg,0,3.1763379771,2.9297970413,-1.0696118795

HF $=-\mathbf{2 2 1 5 . 7 9 5 6 1 7 8}$

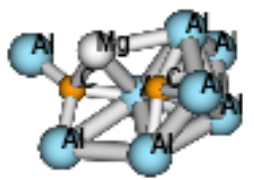

Al,0,0.3526581426,-0.8516625133,-0.3599307317 Al,0,-0.0045435302,1.5761495308,-1.7907920808 Al,0,2.8006604206,0.6417711187,-1.0429019974 Al,0,3.2916821743,-1.3559925119,1.6986637145 Al,0,-1.8265674971,-2.6646958773,0.059534839 Al,0,-2.2280394815,2.2289543368,-0.204956285 Al,0,-1.8194077034,-0.1196451893,1.3565416438 Al,0,-1.9704758281,-0.3949680576,-1.487187443 C,0,2.1655995202,-0.4415859741,0.4830253025 Mg,0,0.7922808406,0.786763418,1.6746214027 C,0,-0.7080890451,0.9049189913,0.0049599636 HF $=-\mathbf{2 2 1 5 . 7 8 8 6 1 9 1}$

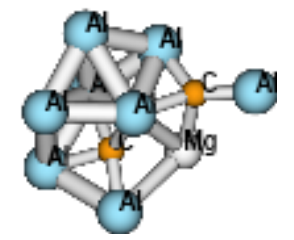

Al,0,-0.3402601608,0.1509776602,-1.0551884585 Al,0,0.9766126135,0.7246081157,1.401922137 Al,0,2.9648621592,-0.8481540012,0.6258567529 Al,0,1.2421680376,-2.6852482638,-0.6390631922 Al,0,-3.98019922,-0.6540358506,-0.4159453123 Al,0,2.1749343188,1.1849969517,-1.1654330202 Al,0,-1.5904313653,1.4146508634,0.9310953543 Al,0,0.2048321819,2.8846291664,-0.3289882813 C,0,-2.1901007176,-0.096601427,-0.1718076157 C,0,0.8803359386,-0.944277054,0.260902287 Mg,0,-1.1353460558,-1.8330207882,0.6550086863 HF $=-2215.788807$

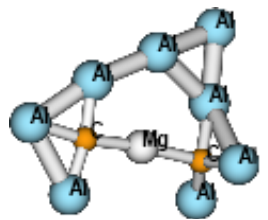

$\mathrm{Al}, 0,-0.7644233899,-2.2921660816,0.1225343125$ 
Al,0,1.7998740225,-1.8170814284,0.1709777119

Al,0,-2.3662205438,-0.2471622864,-0.2024599755

Al,0,-3.2166278795,-2.6368214367,-0.4368738643

Al,0,-1.7272360695,2.87897763,-1.3590300349

Al,0,-2.0771307075,1.8621593203,1.8783780797

Al,0,3.3202682687,1.7556260071,-0.1201807107

Al,0,4.2405846343,-0.8800806004,-0.0298110002

Mg,0,0.4257415769,0.6857288074,-0.0425904953

C,0,-1.5596592169,1.564496176,0.0218515546

C,0,2.4218180036,0.0465687745,0.0123379796

HF $=-\mathbf{2 2 1 5 . 7 8 7 4 0 2 2}$

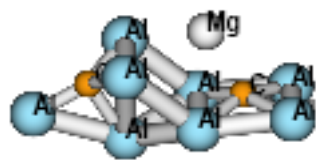

Al,0,3.418416294,-1.4331447852,-0.2963852754

Al,0,0.6622074047,-1.5487775459,-0.0128616018 $\mathrm{Al}, 0,3.4539265521,1.1352617361,0.7280898268$ Al,0,0.686289413,1.0140355364,1.1142833296 Al,0,-1.4350307987,-0.6191722373,1.4217837101 Al,0,-1.5446888192,1.8023880803,-0.3826100414 Al,0,-1.5320742813,-0.8454709346,-1.572385433 Al,0,-4.2032654328,-0.3159116749,0.7923863631 C,0,1.9975928091,-0.0376742061,0.0009703287 C,0,-2.6035724535,0.1055480201,-0.1730758752 Mg,0,0.8383944628,0.8444209038,-1.8556065112 HF $=-2215.812097$

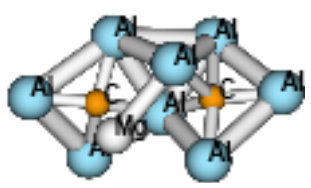

Al,0,1.6050950522,-1.6890370878,1.3039966877 Al,0,1.5797075641,2.0338269367,-0.4399858171 Al,0,1.0812773312,-0.8948315802,-1.4714510762 Al,0,3.6692006368,0.1456423117,0.6050328484 Al,0,-3.552683523,-0.3979681971,-0.7221802105 Al,0,-1.0734778689,-1.9906214444,0.030830017 Al,0,-0.0962888885,0.3441598336,1.1770955672 Al,0,-2.4562295021,1.4051126758,1.1933354113 C,0,1.7200083163,0.1302662444,0.1751797206 C,0,-1.711271179,-0.0659310298,-0.1436573621 Mg,0,-0.8240194372,1.0985253237,-1.8321573926 HF $=-\mathbf{2 2 1 5 . 7 9 7 5 4 1 8}$ 


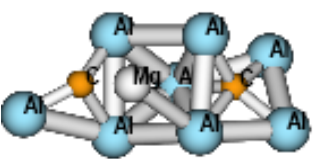

Al,0,-0.4986666501,-0.7224682958,-1.3824083892 Al,0,0.1465585432,1.7591769786,-0.1175063444 Al,0,2.0334457496,-0.27060859,-0.6475420293 Al,0,3.8899545534,-1.6760456394,1.1333549366 Al,0,0.2644116974,-2.1243104492,0.8400950982 Al,0,-2.0497534716,2.7418157811,-1.4135761541 Al,0,-1.7327846192,-0.0345811312,1.1358199421 Al,0,-3.1649862854,0.1789622679,-1.7445885667 Mg,0,1.0003039407,0.3677631434,2.1513865117 C,0,-1.6111938462,0.8926495647,-0.6947804098 C,0,2.0195303442,-1.3073811822,1.1507689846 HF $=-2215.806421$

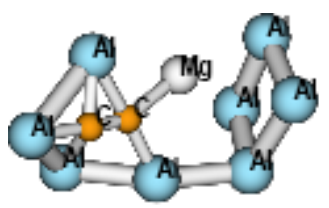

$\mathrm{Al}, 0,3.4343763411,1.5415944481,-0.624409847$ Al,0,3.2222083984,0.3619568098,1.7383245312 Al,0,2.6835891922,-0.7537579378,-1.4820988637 Al,0,2.0695757178,-1.8279038952,0.8343387547 Al,0,-4.5035554182,0.4612562358,0.6865538778 Al,0,-2.0976648714,2.163014047,-0.2164978878 Al,0,-0.6087502642,-1.7331943988,0.3047995914 Al,0,-2.9883293798,-1.3251234537,-0.9823898763 Mg,0,0.6290743383,0.9817773252,-0.511200755 C,0,-1.2799798272,0.1817113383,0.0599191721 C,0,-2.6029765674,0.2644099915,0.4021383973 $\mathbf{H F}=-\mathbf{2 2 1 5 . 7 7 0 2 2 3 9}$

Al,0,-2.3315442166,0.9977204712,-0.6308816647 Al,0,1.327712536,-2.0642412641,-0.240264832 Al,0,-1.3145533005,-1.8808382285,-0.6346838297 Al,0,-3.6569703654,-1.0860320682,0.2417182466 Al,0,-0.9771919587,-0.1233627025,1.5316127572 Al,0,1.7815217049,0.970337314,-1.3000480669 Al,0,1.4946998297,0.6454857631,1.4161429866 Al,0,-0.3942735941,2.870005147,-0.0784509914 Mg,0,4.3223484468,-0.6413707187,-0.0087909751 
C,0,-0.1457199599,0.9405302017,-0.3026581353

C,0,0.3206550229,-0.3707833671,-0.3409065602

HF $=-\mathbf{2 2 1 5 . 7 9 3 3 1 2 3}$

Al,0,-2.6232783928,-1.0623589041,-1.5608456668 Al,0,-2.3664595835,0.075465428,2.1283412045 Al,0,2.9900928628,0.8139727651,0.8158549135 Al,0,2.0594244977,-1.7124871343,1.0436469403 Al,0,0.061815131,-1.6564380734,-0.8260810983 Al,0,-1.688398941,1.8223540056,-0.2656186037 C,0,-2.6147731216,-0.017652188,0.132194564 C,0,-1.2575292102,-0.4589721739,0.0685353467 Al,0,2.1821659067,-0.3372392914,-1.5890638408 Al,0,0.277439146,0.3908764501,1.2057090097 Mg,0,0.9689504867,2.0429881649,-1.1316363855 $H F=-2215.7667686$

Al,0,-0.5026434849,1.9221916869,-1.0851951787 Al,0,-0.4546929062,1.0437167099,1.8263556872 Al,0,1.8277753371,1.3686937227,-0.1265670802 Al,0,1.820987918,-1.3969175189,0.1120011015 Al,0,-2.8047724295,-1.3865542608,-0.0806447523 Al,0,4.1795535318,-0.0318540876,-0.0729986589 Al,0,-0.5159877297,-1.7414802557,1.2705510901 C,0,0.2926122124,0.0041232867,0.0536026552 Mg,0,-0.3333720209,-1.2314331999,-1.9491912876 Al,0,-2.8501382162,1.3578451796,-0.0551882329 C,0,-1.142712548,-0.0018127685,-0.0299003611 HF $=-2215.7954751$

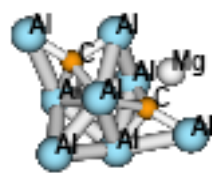

C,0,1.5312939259,-0.3244859817,0.3338095691 Al,0,-1.0477110824,-2.1062972439,0.4074290289 $\mathrm{Al}, 0,0.4058934338,-0.4843302886,1.9628025949$ Al,0,-1.5248762364,1.4220013422,0.7035007859 Al,0,-3.730133448,-0.3857782169,-0.3674235791 Al,0,3.4984456562,-0.3836196164,0.3647434915 
Al,0,-0.3101491895,2.7637900023,-1.489027325

Al,0,1.4182974869,1.6870459441,0.2640731826

C,0,-1.8266279286,-0.3440746138,-0.1403965367

Mg,0,1.6109750212,-2.3663302838,-0.8036478941

Al,0,-0.0605124852,-0.0199406168,-1.1935369077

HF $=-2215.781454$

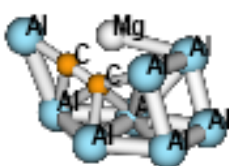

Al,0,-0.0914721691,-2.3994564027,0.3385343031 Al,0,2.4597453158,-1.5044955711,0.5398241975 Al,0,0.765301043,0.2989319678,1.7932474268 Al,0,3.1522588939,0.4570819036,-0.938662635 Al,0,-1.9664882303,-1.0977333935,-1.3142186426 Al,0,0.6105660812,-0.1311565561,-1.1424682546 Al,0,1.5339042562,2.3023393923,0.0917043336 Mg,0,-1.2799465603,1.977024596,-0.1792213113 Al,0,-3.9541623835,0.4074162559,0.3221757404 C,0,-0.767218306,-0.2999499988,0.493723953 C,0,-2.110469656,-0.0421089852,0.5360896533 HF $=-\mathbf{2 2 1 5 . 7 9 5 9 8 5 8}$

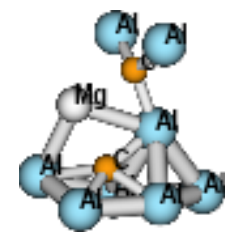

Al,0,-0.1175381087,0.6858947666,-0.8521374814 Al,0,1.8268288968,3.1031340505,0.6687913129 Al,0,2.9048360192,1.3500457841,-1.8340231463 Al,0,-0.1933866823,-2.99233368,0.6017723404 Al,0,-0.3771032766,-1.2108210282,2.5981707121 Al,0,-2.7560370448,0.6301132858,-1.7468076254 Al,0,-2.1540035622,-0.2078582756,0.8732671697 Al,0,-1.2151325445,-1.617355776,-1.4146703045 Mg,0,1.5509070814,0.0156421726,1.2377120177 C,0,1.6546371379,1.5244057776,-0.4002435861 C,0,-0.2464559773,-0.994131565,0.3203664329

\section{HF $=-\mathbf{2 2 1 5 . 7 9 2 0 6 5 4}$}

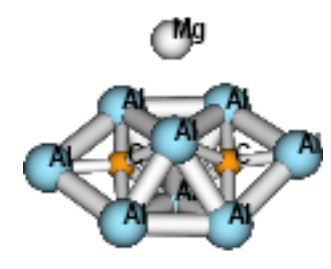


Al,0,-1.1725144562,-1.0877438966,-2.1671318267 Al,0,-1.6764464317,0.3511641641,0.1922225961 Al,0,-0.0490469657,-1.5807694708,1.7353942111 Al,0,-1.0582443204,-3.3377442083,-0.3455023795 Mg,0,2.8716524657,-0.7178732966,1.4265329185 Al,0,0.8262104183,3.3664130302,-0.0441312936 Al,0,0.946966291,-0.1389271227,-0.694610768 Al,0,-0.7150575388,1.7895275774,-1.9116526264 Al,0,0.5158983398,1.2335787478,1.9577811928 C,0,0.0129839506,1.5401452537,0.0715956239 C,0,-0.5947804439,-1.3946461065,-0.1564611902 HF $=-\mathbf{2 2 1 5 . 8 0 4 3 3 4 7}$

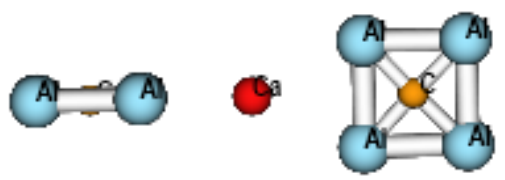

C,0,0.,-3.0403259733,3.0403259733 Al,0,-0.9915564653,-3.3445683256,4.7468203857 Al,0,-1.0067264941,-1.386180465,2.8098858713 Al,0,1.0067264941,-2.8098858713,1.386180465 Al,0,0.9915564653,-4.7468203857,3.3445683256 $\mathrm{Ca}, 0,0 ., 0 ., 0$.

Al,0,1.0067117472,2.8098962989,-1.3861700373 Al,0,-1.0067117472,1.3861700373,-2.8098962989 Al,0,0.9915419406,4.7468306562,-3.3445580551 Al,0,-0.9915419406,3.3445580551,-4.7468306562 C,0,0.,3.0403259733,-3.0403259733

HF $=-2693.2975224$
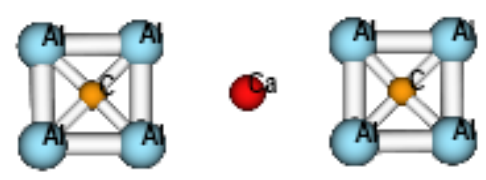

C,0,0.,-3.0600210936,3.0600210936 $\mathrm{Al}, 0,0 .,-3.0705723018,5.0568123849$ Al,0,0.,-1.1104670649,3.1203030739 Al,0,0.,-3.1203030739,1.1104670649 $\mathrm{Al}, 0,0 .,-5.0568123849,3.0705723018$ $\mathrm{Ca}, 0,0 ., 0 ., 0$.

Al,0,0.,3.1203030739,-1.1104670649 Al,0,0.,1.1104670649,-3.1203030739 Al,0,0.,5.0568123849,-3.0705723018 $\mathrm{Al}, 0,0 ., 3.0705723018,-5.0568123849$ C,0,0.,3.0600210936,-3.0600210936 $H F=-2693.2936058$ 


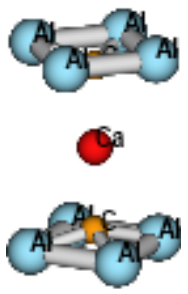

Al,0,-0.0000551494,1.9853767386,2.9708092587 Al,0,-1.9853825725,-0.0000279891,2.9707959437 Al,0,0.0000266789,-1.9853541082,2.9707869106 Al,0,1.9853581616,0.0000535476,2.9707593801 Al,0,1.4037876818,1.4037803855,-2.9709401653 Al,0,-1.4037600449,-1.403805937,-2.9709296119 Al,0,1.403728201,-1.4038626543,-2.9709499845 Al,0,-1.4037031305,1.4038408324,-2.9709089911 C,0,-0.0000165427,0.000014004,2.525150319 C, $0,0.0000165037,-0.0000136989,-2.5240595518$ Ca, $0,0.0000001248,-0.0000006216,0.0000479885$ $\mathrm{HF}=-\mathbf{2 6 9 3 . 2 5 4 8 7 6 2}$
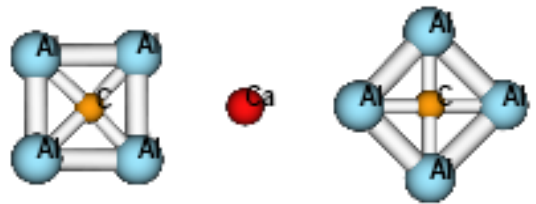

C,0,0.,-3.2210518439,3.2210518439 Al,0,0.,-3.2463014879,5.2230482428 $\mathrm{Al}, 0,0 .,-1.2764537564,3.3108509593$ Al,0,0.,-3.3108509593,1.2764537564 Al,0,0.,-5.2230482428,3.2463014879 Ca, $0,0 .,-0.2158173022,0.2158173022$ C,0,0.,3.3252047662,-3.3252047662 Al,0,0.,1.9735185655,-1.9735185655 Al,0,0.,4.7294071561,-4.7294071561 Al,0,0.,4.7316313767,-1.9060533874 $\mathrm{Al}, 0,0 ., 1.9060533874,-4.7316313767$ HF $=-\mathbf{2 6 9 3 . 2 7 8 5 8 1 7}$

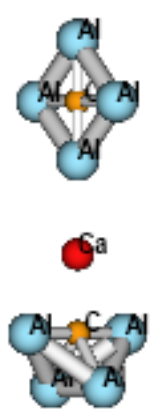

C,0,-3.2365830699,0.,0.

Al,0,-5.0231854111,0.,1.3157840904 
$\mathrm{Al}, 0,-3.2623943619,1.9735025577,0$.

Al,0,-5.0231854111,0.,-1.3157840904

Al,0,-3.2623943619,-1.9735025577,0.

Ca, 0,-0.7049421536,0.,0.

Al,0,2.3672670225,0.,0.

$\mathrm{Al}, 0,6.2631123105,0 ., 0$.

C,0,4.2780579289,0.,0.

Al,0,4.2723129495,0.,1.9981425218

$\mathrm{Al}, 0,4.2723129495,0 .,-1.9981425218$

$H F=-2693.2219879$

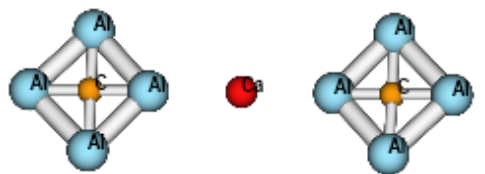

Ca,0,0.,0.,0.

Al,0,1.4146697422,4.5024177016,-2.5017432506

Al,0,-0.0000002021,2.1626127483,-2.1626130341

Al,0,-0.0000004594,4.9147230691,-4.9147237185

Al,0,-1.4146703969,2.5017425234,-4.5024179

C,0,-0.0000003281,3.5097610259,-3.5097614897

Al,0,0.0000002021,-2.1626127483,2.1626130341

Al,0,0.0000004594,-4.9147230691,4.9147237185

C,0,0.0000003281,-3.5097610259,3.5097614897

Al,0,1.4146703969,-2.5017425234,4.5024179

$\mathrm{Al}, 0,-1.4146697422,-4.5024177016,2.5017432506$

HF $=-2693.2591416$
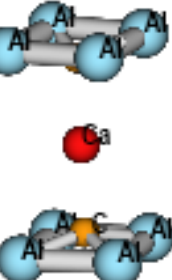

Al,0,1.4886157862,1.3119909593,2.9782144053

Al,0,-1.3119951426,1.4886843269,2.9782464497 Al,0,-1.4887021449,-1.3119271742,2.978163098 Al,0,1.3119240476,-1.4886278871,2.9780949863 Al,0,1.3118151725,1.4886050389,-2.9778183404 Al,0,-1.3117558083,-1.4886503364,-2.9778766442 Al,0,1.4884689945,-1.3116758392,-2.9792058378 Al,0,-1.4884128438,1.3116268199,-2.9792008693 C,0,-0.0000443972,0.000039216,2.5248177313 C, $0,0.0000253005,-0.0000303085,-2.5236756479$ Ca,0,0.0000329891,-0.0000195124,0.0005561641 $H F=-2693.2545113$ 


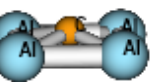

C,0,-3.4152849232,0.,0.

Al,0,-3.8254124021,0.7599389386,1.8400290695 Al,0,-3.8254124021,1.8384550063,-0.7637390631 Al,0,-3.8254124021,-0.7599389386,-1.8400290695 Al,0,-3.8254124021,-1.8384550063,0.7637390631 Ca,0,-0.8911673273,0.,0.

Al,0,2.1684683074,0.,0.

Al,0,6.0622509447,0.,0.

C,0,4.0759088021,0.,0.

Al,0,4.0685268425,-0.7655638738,1.8482346974 Al,0,4.0685268425,0.7655638738,-1.8482346974

$\mathrm{HF}=-\mathbf{2 6 9 3 . 2 5 8 7 4 4 3}$
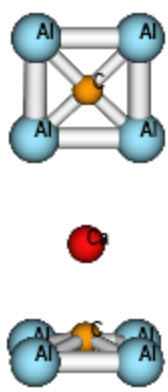

C,0,-3.1391348632,0.,0.

Al,0,-3.5723820852,0.755648732,1.8353688654

Al,0,-3.5723820852,1.8321261153,-0.7634774231

Al,0,-3.5723820852,-0.755648732,-1.8353688654

Al,0,-3.5723820852,-1.8321261153,0.7634774231

Ca,0,-0.5816599053,0.,0.

Al,0,2.3587755105,0.5467836388,-1.3200524713

Al,0,5.1077349865,-0.5365050898,1.295237859

C,0,3.6837671322,0.,0.

Al,0,2.3587755105,-0.5467836388,1.3200524713

Al,0,5.1077349865,0.5365050898,-1.295237859

$H F=-2693.2768667$
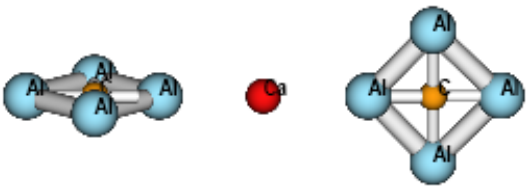
Ca,0,0.,0.,0.

Al,0,1.4147468819,4.4987150274,-2.4979314789

$\mathrm{Al}, 0,-0.0000002026,2.158244578,-2.158244863$

Al,0,-0.0000004609,4.9099471682,-4.9099478165

Al,0,-1.4147475388,2.4979307521,-4.4987152244

C,0,-0.0000003291,3.5055175383,-3.5055180012

$\mathrm{Al}, 0,0.0000002026,-2.158244578,2.158244863$

Al,0,0.0000004609,-4.9099471682,4.9099478165

C,0,0.0000003291,-3.5055175383,3.5055180012

Al,0,-1.414767613,-2.497945544,4.4987006982

Al,0,1.4147682699,-4.4987002355,2.4979460052

HF $=-2693.2604749$
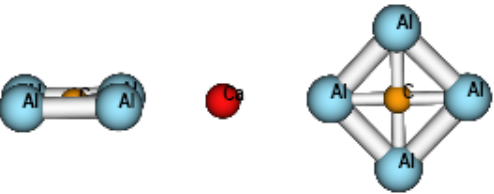

C,0,0.000000301,-3.2139220883,3.213922514

Al,0,-0.9879458886,-3.5290767051,4.9262238273

Al,0,-1.0171379287,-1.5672937792,3.0057235613

Al,0,1.0171383569,-3.0057232585,1.5672940821

Al,0,0.9879466804,-4.9262232673,3.5290772651

Ca,0,0.0000000203,-0.2164512758,0.2164513045

Al,0,1.4128119355,4.3119764513,-2.3139293744

Al,0,-0.0000001841,1.9657458312,-1.9657460915

Al,0,-0.0000004422,4.7218339352,-4.7218345606

Al,0,-1.4128125559,2.313928671,-4.3119766255

C,0,-0.0000003107,3.3175626041,-3.3175630435

$H F=-2693.2806694$
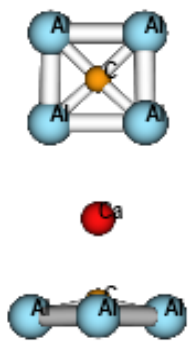

C,0,-3.1403063781,0.,0.

Al,0,-3.5855733523,0.,1.9857225922

Al,0,-3.5565649268,1.98470723,0.

Al,0,-3.5855733523,0.,-1.9857225922

Al,0,-3.5565649268,-1.98470723,0.

Ca, $0,-0.5819340545,0 ., 0$.

$\mathrm{Al}, 0,2.3577626705,0 .,-1.4286864667$

$\mathrm{Al}, 0,5.1067962107,0 ., 1.402022112$

C,0,3.6829306174,0.,0. 
$\mathrm{Al}, 0,2.3577626705,0 ., 1.4286864667$

$\mathrm{Al}, 0,5.1067962107,0 .,-1.402022112$

\section{$\mathbf{H F}=\mathbf{- 2 6 9 3 . 2 7 6 8 4 5 7}$}
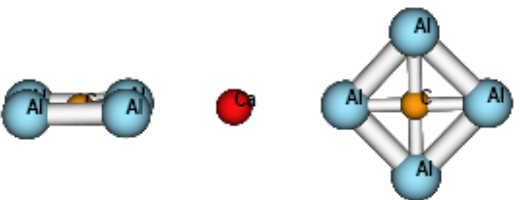

C,0,-0.1916900323,-3.3386873946,3.0806045052 Al,0,-1.2081505277,-3.6701124592,4.7726138515 Al,0,-1.1207414405,-1.6345696051,2.9321282815 Al,0,0.8478263002,-3.1151283759,1.450068114 Al,0,0.7039864066,-5.1111258315,3.3299035922 Ca,0,-0.0129204027,-0.224173277,0.2067582448 Al,0,1.6503466421,4.3251146326,-2.1255224554 Al,0,0.1172171375,2.0416896004,-1.8838693784 Al,0,0.2816851856,4.90355868887,-4.5243192631 Al,0,-1.255144,2.5562343109,-4.2237140366 C,0,0.1978690162,3.4453329027,-3.1789241836

\section{$\mathrm{HF}=-\mathbf{2 6 9 3 . 2 8 0 6 7 3}$}

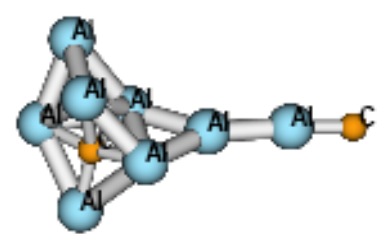

C,0,-0.1255062429,1.8830061027,0.0985418284 Ca,0,5.231038919,-2.3557695535,-0.0544962113 Al,0,-4.5535919731,-2.2861024912,-0.1469430262 Al,0,-2.6323337114,-0.657337759,-0.0378820433 Al,0,-1.7509697748,1.4957776799,1.1853526757 Al,0,-0.2620202998,-0.0154913985,-0.7706398497 Al,0,0.8561684414,1.0854168198,1.726081624 Al,0,-0.8314823217,3.3583531409,-1.0154807141 Al,0,1.6487740404,1.9163936273,-0.8377150755 Al,0,2.3279283131,-0.5502814262,0.0851141906 C,0,-6.049981034,-3.4483520093,-0.3239779841 HF $=-2693.0887313$

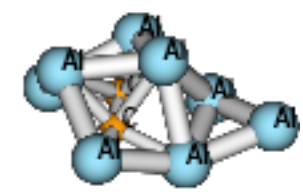

Al,0,0.1375704383,-2.1553101434,-1.6514173171 Al,0,1.1136634973,2.0305541076,-0.1388952115 
Al,0,2.0893736964,-0.0213081907,-1.4310512133 Al,0,2.626162779,-2.5645415468,-1.2043488156 Al,0,0.4485732067,-0.7682703767,0.8066234273 Al,0,-1.4503982606,2.4763116182,-1.0126575661 Al,0,-2.0267312357,0.4289813504,0.7616517742 Al,0,-2.3318450346,-1.8810742409,-0.7478361103 Ca,0,-0.0219771705,1.5156787523,3.5251121473 C,0,-1.0118645312,-0.4190970472,-0.9739759688 C, $0,-0.228677922,0.6852589547,-0.7708806182$ HF=-2693.2571402

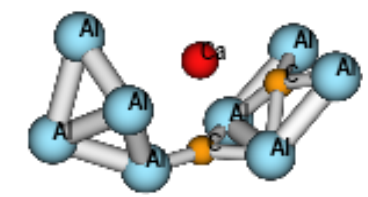

Al,0,-3.7354414501,-0.4378267005,1.8188877372 Al,0,-2.9068163063,0.7924967025,-0.4307601184 Al,0,-1.347837503,0.8480118612,1.7538444622 Al,0,-3.5162058194,-1.6379676214,-0.9355941913 Al,0,3.0587360518,1.2139553299,-2.0388984089 Al,0,3.691155628,-1.4986734255,-0.5728617296 Al,0,1.9998559986,-0.6147650307,1.3818743442 $\mathrm{Al}, 0,1.6116124283,1.8972164053,0.2934388576$ Ca,0,-0.113253765,-0.5408134984,-1.0309727116 C,0,2.4013308465,-0.0121877958,-0.6578882223 C,0,0.4568871432,0.5962631619,1.3429468626 HF $=-2693.2071114$

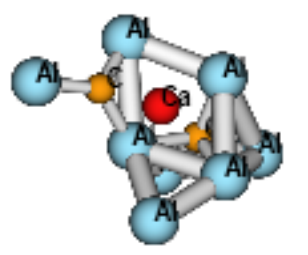

Al,0,0.3242549795,-0.5488538573,-0.809920692 Al,0,-0.0997560197,2.1509465972,-0.5045256395 Al,0,2.2655827633,1.4728339015,-1.3492011233 Al,0,2.2167401128,-3.0895382547,0.7862180363 Al,0,-0.8867303566,-2.1390017682,1.4108231989 Al,0,-2.9220272726,1.8116490997,-1.1890262147 Al,0,-1.672474205,0.5785385475,1.3329890393 Al,0,-1.7610248707,-0.3336306312,-2.4783381982 Ca, 0,1.814818357,0.4285378057,1.8011819495 C,0,-1.4343168821,0.5455190581,-0.6525892802 C,0,0.8783645748,-1.7636896183,0.7174429004 HF $=-2693.2532477$ 


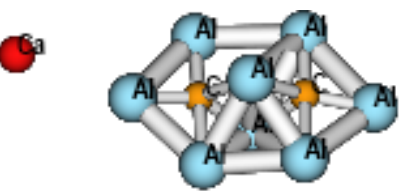

Al,0,-0.8272716014,-2.2242433931,-2.1720940037 Al,0,-1.4434858768,-0.4518441656,-0.270078183 Al,0,-0.7153704112,-2.3975016345,1.9801043298 Al,0,-1.0072767016,-4.3264351089,-0.2241843564 Al,0,0.583905519,2.3428967193,-0.216300046 Al,0,1.147341813,-1.478643479,-0.3031558443 Al,0,0.4510641093,0.3192804023,-2.1311013047 $\mathrm{Al}, 0,0.4455938168,0.4298056322,2.0479154577$ C, $0,0.4194019494,0.3994595455,0.0782458012$ C,0,-0.7133268706,-2.3453565225,0.0045370876 Ca,0,0.9757520428,5.6451143609,0.8129462013 $\mathbf{H F}=-\mathbf{2 6 9 3 . 2 5 8 5 7 0 4}$

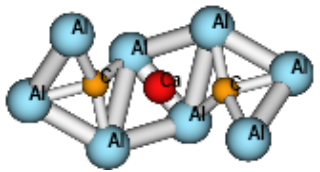

C,0,-1.9840171023,-0.0784182871,-0.0457039692 Al,0,0.9020862818,-1.7551383602,0.4315246473 Al,0,-0.9483813773,-1.0703616246,-1.4282760237 Al,0,-2.7383838635,1.5340411422,-1.0140549051 Al,0,1.4877964669,0.0795790039,-1.9749878304 Al,0,2.7715490325,0.4796540899,1.7240332882 Al,0,-1.505197296,-1.1342640784,1.6032715277 Al,0,3.9518460511,-0.1545561066,-0.6883175749 Al,0,-3.9611776414,-0.439649028,0.4778062491 C,0,1.980964277,-0.146782114,-0.0337217703 Ca,0,0.0268263724,1.6670118455,0.5886781261 $\mathrm{HF}=-\mathbf{2 6 9 3 . 2 3 8 3 2 7 3}$

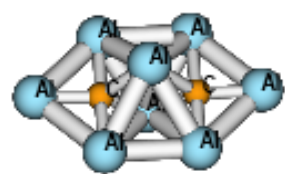

Al,0,-0.3412731093,-1.3080211621,-1.3401736487 Al,0,-1.3690001459,0.3545296351,0.5483559321 Al,0,-0.5080507155,-1.5467718233,2.7678322015 Al,0,-1.0200484694,-3.4029798249,0.6157609112 $\mathrm{Al}, 0,0.9430379147,3.3310447989,1.0068976572$ Al,0,1.3136436241,-0.4049034017,0.6100586442 $\mathrm{Al}, 0,0.4205577174,1.431242609,-1.1831772878$ 
Al,0,0.2846978598,1.2816693655,2.9301246427

C, $0,0.3655294283,1.3970455219,0.9398031428$

C,0,-0.4400048518,-1.4747802855,0.7750189467

Ca,0,0.2020255877,0.1950438014,-4.385638011

HF $=-2693.2640266$

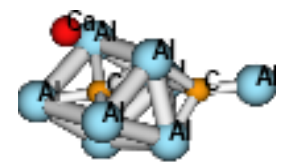

Al,0,1.0883339026,0.1090736753,-1.9347986843 Al,0,0.7819914231,-0.0234824749,2.0790552192 Al,0,0.0140739775,2.4107394616,0.0830996047 Al,0,2.5544376693,1.4400789869,0.2416347444 Al,0,-4.0709698669,-1.2086506373,-0.3523744769 Al,0,-1.5141644454,0.7169951798,-1.5742955005 Al,0,-0.4606943119,-1.3321723158,-0.0792267705 Al,0,-1.7410381225,0.6174848896,1.3724378878 C, $0,0.7200870419,0.4736087039,0.069967523$ C,0,-2.3391172853,-0.4785000057,-0.1941551321 Ca,0,2.6619284263,-1.7730760069,0.1441604672 HF=-2693.273294

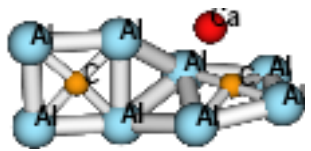

Al,0,3.2032007725,-1.8427476291,0.9660874727 Al,0,0.570744726,-1.2630466142,0.8394347307 Al,0,3.8803614314,0.5585986539,-0.3598708247 Al,0,1.284488346,1.4364920578,0.3501518585 Al,0,-1.0920844325,0.7194129321,1.5623621052 Al,0,-3.7467325881,1.2854893605,1.801707513 Al,0,-1.5450450896,-0.7000191664,-0.785139173 Al,0,-4.283554501,-0.0547279583,-0.6007725681 C,0,2.1644235631,-0.3450646853,0.1167316509 C,0,-2.6533335984,0.3634604302,0.4203420638 Ca,0,1.2702768786,-0.096162287,-2.6141968387 $\mathbf{H F}=-\mathbf{2 6 9 3 . 2 8 1 9 7 4}$

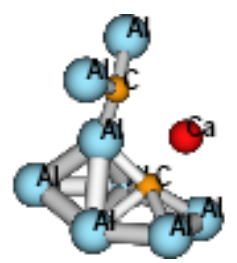

Al,0,-0.4364401566,0.9675260154,0.0053570106 Al,0,-3.3185222388,0.1527492588,-1.5650245718 Al,0,-3.2280979921,0.6822308525,1.6006447057 Al,0,2.4140878884,-1.4224583178,1.3393742647 
Al,0,1.763987064,-1.9802797881,-1.192444081

Al,0,1.2680592477,3.1678225045,-0.3809986394 Al,0,1.9516994064,0.6680828864,-1.3588584536 Al,0,1.7714945955,1.1344362029,1.4043663515 Ca,0,-1.0457743589,-2.1164176026,0.0160463348 C,0,-2.2670741968,0.1991129416,0.0219778134 C,0,1.0160751285,-0.4462917644,0.2442984658 HF $=-2693.2533039$

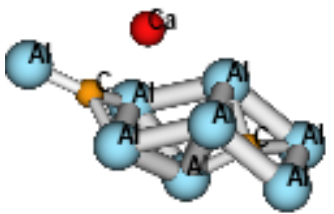

Al,0,-0.4184189239,-0.5541444453,-1.8279705793 Al,0,-0.089819099,1.8232949058,-0.2611642751 Al,0,2.0118940378,0.0165680375,-0.7822080614 Al,0,3.6605433071,-2.0306116417,1.5584239042 Al,0,0.4020228424,-2.1087402409,0.2303312352 Al,0,-2.0739589252,2.4499391094,-2.1525168385 Al,0,-1.6705848657,-0.2632054339,0.7332072044 Al,0,-3.6753869875,0.3348115052,-1.1453883265 Ca,0,1.0734340799,0.3697338799,2.485046308 C,0,-1.7136726358,0.7952506853,-1.0572108635 C,0,2.1519276996,-1.3081725099,0.6761756007

\section{HF $=-2693.2725382$}

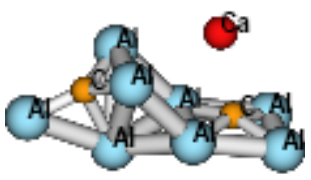

Al,0,3.3529454286,-1.51991122,-0.0694761101 Al,0,0.5738455485,-1.553433309,0.0827005753 Al,0,3.3837866551,0.9960638441,1.0576353283 Al,0,0.604321443,0.9645990772,1.2058811644 Al,0,-1.4826220576,-0.6903836916,1.5873421905 Al,0,-1.6722724462,1.6987358658,-0.2372975932 Al,0,-1.7058906222,-0.922427788,-1.4065406803 Al,0,-4.2775179733,-0.4255696301,1.072522259 C,0,1.9529886611,-0.1000973638,0.1717904526 C, $0,-2.7169669188,0.0136525021,0.0474256498$ Ca,0,1.0244060929,0.9699459121,-2.2060634678 HF $=-2693.2770829$ 
Al,0,3.263120046,1.3177316193,-0.8561566382 Al,0,1.846795007,-0.755506292,1.0171669717 Al,0,-1.3893744976,0.6736811845,-1.4292287676 Al,0,-2.838502023,-1.2581334491,-0.2462165348 Al,0,-0.8911004851,0.0275702708,1.6444409001 Al,0,1.1201557266,-0.4622479673,-2.0761758547 C,0,1.4842372179,0.8231224435,-0.4154947487 C,0,0.2475513825,0.2558324862,-0.1054887788 Al,0,-1.9764151002,-2.5323589524,1.7272427311 Al,0,-0.1863025424,-1.9740029378,-0.3300743718 Ca,0,0.1640189346,2.9024367616,0.513146075 $\mathrm{HF}=-\mathbf{2 6 9 3 . 2 7 5 6 9 1}$

Al,0,-0.0434678763,0.4155650248,-0.6840367034 Al,0,-0.5652496891,3.1560255572,-0.2953103918 Al,0,1.592970149,1.9147753302,1.0222340804 Al,0,3.3696201961,0.1469134908,-1.4955285203 Al,0,-0.7253926303,-3.1093414947,-0.7230346943 Al,0,-2.5797778686,1.5326705803,-0.0984197754 Al,0,-1.4592929846,-0.2484764923,1.6684679251 Al,0,-2.5040483877,-1.024428167,-0.997831968 C,0,-0.7797377658,-1.323679906,0.0818061493 C,0,1.906209051,0.5073763945,-0.3701774089 Ca,0,1.5565740239,-1.5645164356,1.1287604088 HF=-2693.244527 


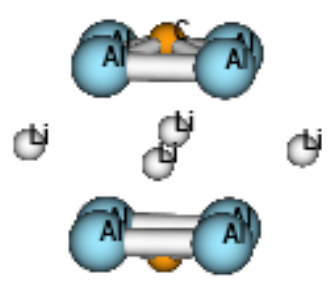

Al,0,1.9135925617,0.,-1.9611442551

Al,0,0.,-1.9135925617,-1.9611442551

Al,0,-1.9135925617,0.,-1.9611442551

Al,0,0.,1.9135925617,-1.9611442551

Al,0,0.,1.9135925617,1.9611442551

Al,0,-1.9135925617,0.,1.9611442551

Al,0,0.,-1.9135925617,1.9611442551

Al,0,1.9135925617,0.,1.9611442551

C,0,0.,0.,2.3803421766

C,0,0.,0.,-2.3803421766

Li,0,2.1359577445,2.1359577445,0.

Li,0,-2.1359577445,-2.1359577445,0.

Li,0,-2.1359577445,2.1359577445,0.

Li,0,2.1359577445,-2.1359577445,0.

HF $=-2045.8695792$

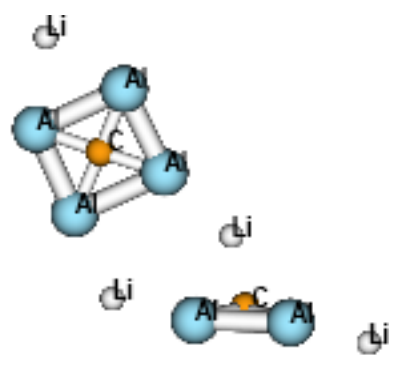

Al,0,-4.20744448499,-1.6087384037,0.

Al,0,-2.8953670804,-4.0818098595,0.

Al,0,-1.8005082329,-0.3350160632,0.

Al,0,-0.490300703,-2.8515757831,0.

C,0,-2.3319568362,-2.1961027754,0.

C,0,2.0231851685,2.1587066613,0.

Al,0,2.4556023547,0.7498637496,1.3836553026

Al,0,2.4029490318,3.4850308103,-1.4120927271

Al,0,2.4556023547,0.7498637496,-1.3836553026

Al,0,2.4029490318,3.4850308103,1.4120927271

Li,0,-5.6049844217,-3.9383108094,0.

Li,0,1.9442859977,-1.6676384604,0.

Li,0,2.908893611,5.7717010889,0.

Li,0,-0.0324083634,1.6742280317,0.

\section{HF $=-\mathbf{2 0 4 5 . 8 5 9 7 5 8 9}$}


bi

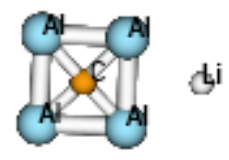

Li,0,-0.3258228959,-1.6085119024,-0.0173840808

Li,0,0.3257625921,1.6089564463,0.0174511179

Li,0,7.5880173439,-0.8831344457,-0.0051573511

Li,0,-7.5879463953,0.8834113599,0.0049156679

Al,0,4.9913718888,-1.7139305607,-0.0153379367 Al,0,3.0518317515,1.6919347771,0.0199054646 Al,0,5.6549400049,1.0236067384,0.0141430749 Al,0,2.3280513308,-1.0042143538,-0.0092137872 Al,0,-3.0519796099,-1.6920439646,-0.0202569959 Al,0,-4.9912267561,1.7140042778,0.0157197941 Al,0,-5.6550294503,-1.0234872572,-0.0146494712 Al,0,-2.3279532242,1.0039682831,0.0096865393 C,0,3.9953297017,-0.0298338978,0.0020987656 C,0,-3.9953478842,0.0298242984,-0.0020042536

\section{HF $=-\mathbf{2 0 4 5 . 8 6 1 8 5 0 8}$}

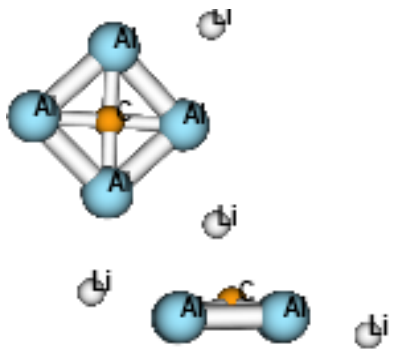

Al,0,-4.260080724,-2.3309490338,0.

$\mathrm{Al}, 0,-2.0995308334,-4.1206513505,0$.

$\mathrm{Al}, 0,-2.5097852578,-0.1776977492,0$.

$\mathrm{Al}, 0,-0.403991775,-1.8643876225,0$.

C,0,-2.3204269956,-2.1271540429,0.

C,0,2.0010900834,1.852005178,0.

Al,0,2.6224859718,0.518051588,1.3798878906

Al,0,2.1641726941,3.2200308791,-1.4120407117

Al,0,2.6224859718,0.518051588,-1.3798878906

$\mathrm{Al}, 0,2.1641726941,3.2200308791,1.4120407117$

$\mathrm{Li}, 0,-5.2575026026,0.1604810503,0$.

Li,0,2.3017561187,-1.8707310533,0.

Li,0,0.0001087403,1.1137442926,0.

$\mathrm{Li}, 0,2.2946203551,5.5560603344,0$.

HF $=-\mathbf{2 0 4 5 . 8 5 5 2 5 4 9}$ 


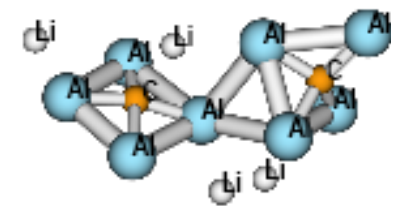

C,0,-2.3594443616,0.1552294571,-0.2592172446

Al,0,-2.687843368,-1.5965273615,0.5526132809 Al,0,-0.5457464504,0.4189343229,0.5559558791 Al,0,-2.4017273265,2.1543291588,-0.450513232 Al,0,-4.2711314461,0.3142740444,-0.772754265 Al,0,4.3834859344,-1.3128967493,-0.9521262953 Al,0,2.0061229533,1.3961607053,0.1217209288 Al,0,1.3754766421,-1.0655397064,-1.0497323586 Al,0,3.0922410965,-0.5574153509,1.8981563507 C,0,2.9252936069,-0.3771638293,-0.0345466881 Li,0,-5.3035593966,-2.0893359866,-0.0921978398 Li,0,0.1093473729,3.1450858247,0.1070228265 Li,0,-1.1191908961,-0.6403448424,-1.8294985898 Li,0,1.0612329425,1.1060811412,2.8211468844

\section{HF $=-2045.8509113$}

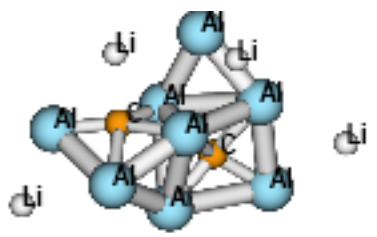

Al,0,0.2479175117,3.0437444845,-0.1559684687 Al,0,-0.1068760294,0.0996938032,1.2805953496 Al,0,-1.9008255384,-1.7658608773,1.0418502908 Al,0,-3.3557446996,-0.4751751825,-0.902676851 Al,0,3.1530439348,-1.1243436307,-0.4340273093 Al,0,-0.1651811348,0.690329258,-1.2975057465 Al,0,2.1928326444,1.329381067,0.3324324131 Al,0,0.3368653018,-2.1602014262,-0.4301978125 C,0,1.3092995675,-0.4131910581,-0.4210822979 C,0,-1.6794378519,0.0284996803,-0.1139558556 Li,0,-2.2214570066,2.0272653955,-0.0473464072 Li,0,-4.2086873813,-2.7882498424,0.2628948519 Li,0,0.6656572155,2.5234147371,2.3954224938 Li,0,4.762625116,0.5774933168,0.9095972851 HF $=-\mathbf{2 0 4 5 . 8 4 3 4 1 4 8}$

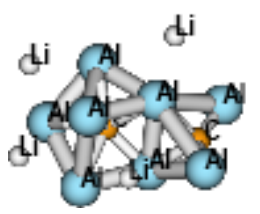

Al,0,-1.4280679668,-0.3620471462,1.4989825419 
Al,0,0.7691910541,-0.9758250087,-1.1816544022 Al,0,-1.1478102243,2.1830618926,0.2947654812 Al,0,-2.7197467627,0.5066844258,-1.0291671024 Al,0,2.6314667331,-1.8119964682,0.8672086625 Al,0,0.8672255493,0.7290569497,0.7805721506 Al,0,-1.5317639147,-1.8647950448,-0.9641898719 Al,0,3.1668299071,1.4350732541,-0.7493716332 C,0,2.3432272935,-0.2402060531,-0.2271888202 C,0,-0.7695128446,0.2173812024,-0.5545533004 Li,0,1.3925096606,3.4286957818,0.2442271698 Li,0,-3.7010028004,-1.5745015029,0.4995050217 Li,0,-3.4957069355,1.5172092126,1.3778351192 Li,0,0.0250322186,-2.629009493,1.5342850156

\section{HF $=-\mathbf{2 0 4 5 . 8 5 9 2 7}$}

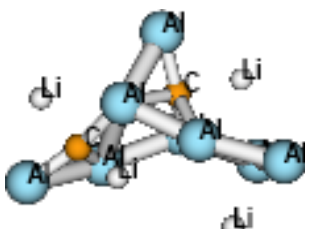

Al,0,3.1482072694,0.6460491542,0.4593502296 Al,0,3.7018889076,-0.657965023,-1.6567630871 Al,0,1.018705713,-0.6032973634,-1.0273960813 Al,0,-0.5227028251,-2.7072360381,1.6634536835 Al,0,0.78990165,0.7211506095,1.5036286268 Al,0,-3.9024428383,2.0135584129,-0.8832944651 Al,0,-1.5448819966,1.831896148,0.7461927127 C,0,0.2632879643,-1.0742695133,0.8764859611 Al,0,-1.4753470269,-1.0013207655,-0.193966784 C,0,-2.5521467179,0.69270415,-0.4598094797 Li,0,-4.0229210141,-0.7446319082,-0.3151185165 Li,0,2.5391370016,1.6923590959,-2.1005644129 Li,0,-1.3588651733,0.5621740704,-2.2197725018 Li,0,2.1626083297,-1.7990561147,1.1535481826 HF $=-\mathbf{2 0 4 5 . 8 3 3 5 9 4 6}$

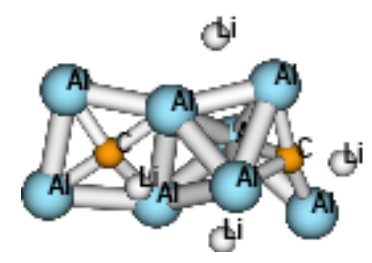

Al,0,-0.8213791994,0.3276666512,-1.696153461 Al,0,-1.937343098,2.0882936001,0.3540370198 Al,0,-3.3181232982,-1.4146261114,-0.7796488017 Al,0,-1.4482109032,-0.5254875632,1.0961723148 Al,0,3.3310123491,1.0437840161,1.3441572292 
Al,0,0.8050175267,-1.4250397513,-0.3140531989 Al, $0,0.702888845,1.1772450333,0.4017841021$ Al,0,3.6795426438,-1.4669161232,0.0139305618 C,0,-2.4778996181,0.2983361711,-0.5119199483 C,0,2.2279455479,-0.0917766081,0.1151560827 Li,0,-0.837572027,1.7357848239,2.7340768491 Li,0,-1.3208715399,-3.0885503527,0.0383040859 Li,0,-3.5268506123,1.3078580295,-1.8688660395 Li,0,1.8804479008,0.4771361162,-1.9309654842 HF $=-2045.8761611$

di

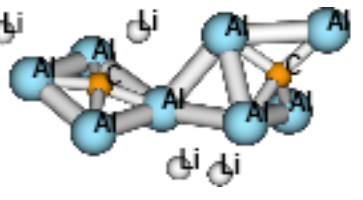

C,0,-2.372520085,-0.1206987134,-0.1177286951 Al,0,-2.4805647714,-1.5908728831,1.1719955974 $\mathrm{Al}, 0,-0.5578840886,0.5123685999,0.4560585791$ Al,0,-2.6259951834,1.7315940903,-0.85174464 Al,0,-4.3124274484,-0.2762791513,-0.511188255 Al,0,1.4233369089,-1.2012427731,-0.8114368757 Al,0,1.8586579374,1.5350569116,-0.4214478332 Al,0,3.2179363584,0.2643881719,1.7440213303 Al,0,4.4418794714,-1.1616636207,-0.8694261288 C,0,2.9425668287,-0.1271225794,-0.1444545555 Li,0,-5.0596591209,-2.4659642475,0.8862440059 Li,0,-0.203139125,3.0442396154,-0.7787614904 Li,0,1.0766419341,1.9481427661,2.3139501368 Li,0,-1.1353403074,-1.2219560449,-1.4933371723 HF $=-2045.8509115$

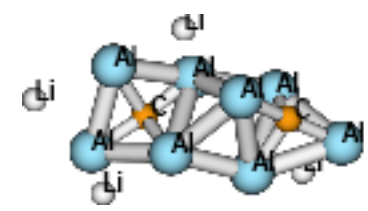

C,0,-2.4466155574,0.1873680349,-0.6846831207 Al,0,-4.1718677331,-0.5853040949,-0.2088235351 Al,0,-2.2456719064,0.7202855426,1.2972620194 Al,0,-1.4548822478,-1.5786647463,-0.3779653715 Al,0,-0.9081854974,0.9701535179,-1.4897032018 Al,0,2.8488202044,1.5442720372,0.2449357661 Al,0,1.0852902993,-0.9601477964,-0.8704136131 Al,0,3.3705649137,-1.1873753671,0.5987496773 Al,0,0.3550665043,1.1421841744,1.0614263504 C,0,1.7916105473,-0.0840933444,0.858880236 
Li,0,5.2770600598,0.5075442642,-0.3455853202

Li,0,3.2233353676,-2.1053313644,-2.0119832939

Li,0,0.8789839266,2.9650792688,-1.031623287

Li,0,-3.2122856609,-1.857255709,1.933769273

HF $=-2045.820506$

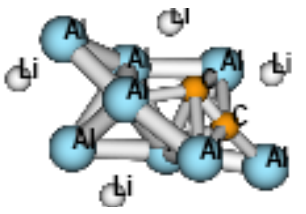

Al,0,-1.2215203926,-2.2132719222,-0.3027401257 Al,0,3.2647528163,-0.5525656311,0.9144952204 Al,0,-3.4262547201,-0.014878122,-0.9008910541 Al,0,1.3302879366,-1.4209881152,-0.661288893 Al,0,-0.5980287363,0.4530819021,-1.2110760136 Al,0,0.9789764704,0.7671451207,1.1163755266 Al,0,1.9741726017,1.4286127811,-1.2758600214 Al,0,-1.6201132058,1.827839857,1.2191716095 C,0,-0.7394711817,-0.4228064767,1.0320578821 C,0,-1.9334643957,-0.0481473403,0.3792949357 Li,0,4.1274694601,-0.2858222015,-1.4838697269 Li,0,0.575577965,-1.7230042378,1.926633589 Li,0,0.3643365217,3.1980024838,-0.1392921816 Li,0,-2.6780281298,-1.4388305152,1.6483489393

\section{HF $=-2045.8522625$}

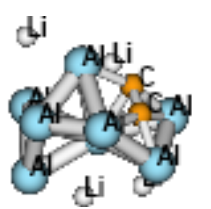

Al,0,-0.1144457403,-0.0069928968,0.9034430397 Al,0,2.3742398874,1.2792735464,0.8236079213 Al,0,-2.7396827343,0.8738744861,1.1530196571 Al,0,2.3580521641,-1.3073513419,1.3688430183 Al,0,-0.4279053132,-1.2639375484,-1.7140529766 Al,0,0.2940442095,1.6194377391,-1.5562941288 Al,0,1.937068262,-0.5529081646,-1.1792211623 Al,0,-2.3004791517,-1.7522643984,0.0668477242 C,0,-1.445028589,1.3401474841,-0.4088344972 C,0,-1.7372941711,0.0114667033,-0.6115221203 Li,0,2.3493702962,3.1736420992,-1.0441490252 Li,0,-0.6730272219,2.8589154704,0.5441663919 Li,0,-1.6570144897,-1.058256371,2.794727516 Li,0,0.3614534074,-2.8637657328,0.3257982832 HF $=-2045.8429882$ 
Al,0,0.0227791984,-0.3130385981,-0.477716657 Al,0,-2.8825925376,0.0666213967,-0.7304883501 Al,0,1.9503242418,-2.3092045614,-1.5034430407 Al,0,-1.286515348,2.1473558736,-0.8734330756 Al,0,0.4344560536,-1.2094489946,2.2550970588 Li,0,-1.2461647168,1.0808572509,1.8240740275 Li,0,0.1877287236,-3.3317003894,0.3337121202 Li,0,1.3256407519,1.268060216,-2.2837048029 $\mathrm{Al}, 0,2.4026634223,0.4785729063,0.2165813723$ $\mathrm{Al}, 0,0.8098378391,2.3880610016,0.651809535$ C,0,0.7214988129,0.4877752887,1.3808230822 Li,0,-3.5911981221,2.0692864542,-2.2904109961 Al,0,-1.902351815,-1.5214675172,1.0276785554 C,0,1.9185289174,-0.4405053196,-1.399176619

\section{$\mathrm{HF}=-\mathbf{2 0 4 5 . 8 4 0 3 2 3 6}$}

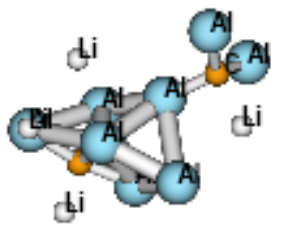

Al,0,1.3507348492,-1.5168206034,-0.7179889047 Al,0,1.0227036589,0.8761240661,-1.9807356896 Al,0,1.2855079075,1.056041468,0.8089480486 Al,0,3.5643615737,-0.5829440265,0.5521148685 Al,0,-3.1548009228,-0.7371334835,2.2203898965 Al,0,-3.5605874864,-1.6616330844,-1.019924768 Al,0,-0.85317664,-0.4055333815,0.1289270967 Al,0,-0.5890193639,2.4475454469,-0.6146047171 C,0,2.4478048349,0.1984935572,-0.8556431211 C,0,-2.7461911618,-0.5389195012,0.3417629609 Li,0,3.2030330628,1.0398697748,2.6638450457 Li,0,1.2935059366,-1.5356738311,2.0203076191 Li,0,-3.1014805737,1.4304235655,-0.1839848433 Li,0,3.2502453976,2.0184313045,-0.7732861016 HF $=-\mathbf{2 0 4 5 . 8 5 5 4 2 8 1}$

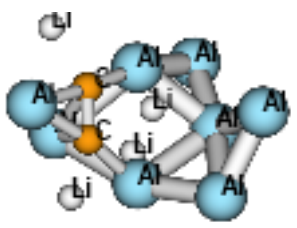

Al,0,-0.2375212371,-1.6857103018,-0.16550872 
Al,0,1.9952306903,-0.3736689321,0.5893967247

Al,0,-2.2777973032,-0.3062029481,1.3436938933 Al,0,2.9716935944,0.4575107768,-1.6480860558 Al,0,-3.3079736508,0.6647538528,-1.4914521029 Al,0,1.5193394912,-2.9503735498,1.2958310155 Al,0,2.0361943281,2.3775298782,-0.1852512267 Al,0,-0.0806861227,1.4384003564,0.9190964962 C,0,-1.6788482314,-0.4504457312,-0.818068303 C,0,-1.6064154423,0.9313264814,-0.3287889534 Li,0,-0.0311656399,0.6797387527,-1.7180998835 Li,0,-2.7415382939,-2.007026153,-1.3640171728 Li,0,-2.0557867399,2.8287012702,-0.2445260707 Li,0,0.052272264,-0.8262116111,2.7702375347

\section{$\mathrm{HF}=-\mathbf{2 0 4 5 . 8 3 3 7 1 0 8}$}

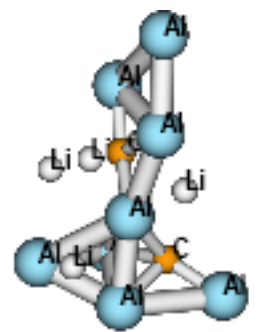

Al,0,-2.0427614058,-2.3604243086,-0.4918731096 Al,0,-2.9779400524,0.070727426,-0.7935513212 Al,0,3.0183764533,-0.7815686563,1.0873170735 Al,0,-1.8567776159,-0.6103625301,1.5946361984 Al,0,2.1467180582,0.7503039509,-0.9142195153 Al,0,-0.3791428447,0.0914261217,-0.6663258891 Al,0,-2.5728269392,2.8268062763,0.2228113885 $\mathrm{Al}, 0,4.949827901,0.5382656369,-0.5733517804$ C,0,1.2238724116,-0.4472420019,0.4772134135 Li,0,0.0966040144,1.3282943267,1.4978596334 Li,0,-1.5775566255,-1.1674270019,-2.8454083554 Li,0,0.5616732027,-1.4651027888,2.2076695928 Li,0,0.7122348104,-2.267377917,-0.5451011068 C,0,-1.7388761471,1.0951718728,0.5234834408

\section{HF $=-\mathbf{2 0 4 5 . 8 4 0 6 3 6 5}$}

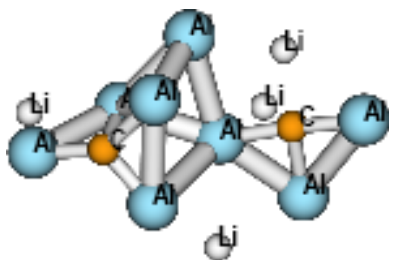

Al,0,1.2383459325,1.9599108692,0.4580124238 Al,0,1.0615070592,-0.545294783,1.7871117099 Al,0,1.8252165946,-0.9439106391,-0.7054519709 $\mathrm{Al}, 0,4.2514776871,0.3047862896,0.2141493817$ 
Al,0,-4.5013399667,0.0395795421,0.2402006844

Al,0,-2.8311681657,1.577689116,-1.4507951869

Al,0,-0.7159011221,0.0523267684,-0.7959737494

$\mathrm{Al}, 0,-0.0690397629,-2.4782354333,0.5395900067$

C,0,2.4240109383,0.4572138628,0.7416338308

C,0,-2.629277976,-0.0208287947,-0.2692637374

Li,0,-0.4795259075,2.8350459976,-1.4847769515

Li,0,-1.8173587818,-0.2074657462,1.6724537521

Li,0,-2.5641181745,-2.1035357743,-0.3242618952

Li,0,4.1487778299,-1.2531721098,-2.0511427215

\section{HF $=-\mathbf{2 0 4 5 . 8 5 2 7 5 5 4}$}

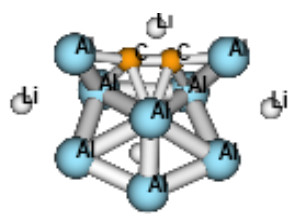

Al,0,2.8081466265,-1.4889667853,-0.4570383088 Al,0,-0.5126454293,2.5334680922,-1.1689534318 Al,0,-0.0859259929,0.0980679172,-0.8444479809 Al,0,2.0307408148,1.9530206863,-0.7779078684 Al,0,1.6141757669,0.0984742042,1.4283230737 Al,0,-2.6445613833,1.1839775837,-0.3962749655 Al,0,-2.2201768113,-2.3169773199,-0.0479829551 Al,0,-1.3156256515,-0.3831303856,1.6674371091 C,0,0.963228418,-1.4861037387,0.1399137211 C,0,-0.4025178865,-1.7108282206,0.2530600655 Li,0,0.4231214106,-3.0793665048,-1.0183233471 Li,0,4.12346857,0.7738159372,0.281589066 Li,0,-0.199542593,2.0036127447,1.5906148346 Li,0,-4.0563561909,-0.5752455609,0.9465016264 HF $=-\mathbf{2 0 4 5 . 8 6 4 3 3 9 6}$

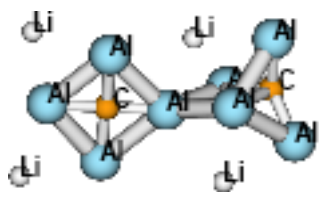

C,0,-1.9254925383,-2.5532374244,0.5769697878 Al,0,-0.9568744702,-4.0699256958,-0.1369605258 Al,0,-0.864134325,-1.5334367855,1.8663356844 Al,0,-3.5264359677,-1.5518419117,0.116391208 Al,0,-1.0901246641,-1.4647990975,-1.1965616695 Al,0,0.4659122733,0.0766418534,0.145584452 $\mathrm{Al}, 0,2.4328932463,3.5150475704,0.0891703186$ C,0,1.487481873,1.7929596757,0.1752031535 Al,0,3.2082549842,0.8969134382,0.3284471062 

Al,0,-0.1845333366,2.7588380303,-0.0791464081
Li,0,1.2585248528,-2.6605042924,-1.1935935416
Li,0,-2.3420651141,0.8976952223,-0.7590221208
Li,0,0.4812671969,5.4046137537,-0.2330490286
$\mathrm{Li}, 0,5.0917253182,2.8333453744,0.300231584$

\section{HF =-2045.8228805}

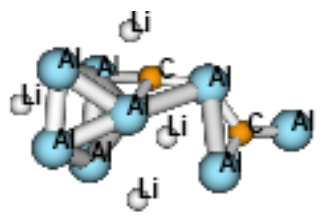

Al,0,3.0479150452,0.5880951467,1.4152692833

Al,0,-4.9176546345,0.2470181857,-0.5880099652 Al,0,-2.364374665,0.5220624635,1.8279623968 Al,0,3.1554868496,-1.5062555714,-0.0723775754 Al,0,1.4152775546,2.0115633662,-0.0353408941 Al,0,-1.9196690689,-1.115208816,-0.6580058594 Li,0,-1.038289712,1.1909020118,-0.7682572102 Li,0,0.9348725905,-2.6486903189,-1.0616729059 Li,0,0.1557257026,1.5517173949,2.3966089274 Li,0,3.7861600202,0.8927574858,-1.2462003826 C,0,-3.1296062942,0.070917213,0.1245237722 Al,0,1.4293924817,-0.0275106639,-1.9936010375 Al,0,0.7748678252,-0.5716840105,0.6978303535 C,0,-0.1356510134,-0.885100717,-1.0711708407 HF $=-2045.8335388$

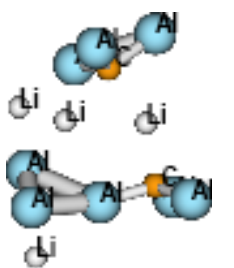

Al,0,-2.5166007603,-1.0791577457,1.1781035287 Al,0,3.662051955,-0.2555161849,-0.4222773527 Al,0,-0.7386778805,-3.477359282,1.5136328078 Al,0,2.3526011491,1.1208229408,2.3199928991 Al,0,-1.6746382028,2.3259332592,-0.3539022709 Al,0,-1.4169590777,-3.0247963039,-1.5156809308 Al,0,1.0665170068,1.647819169,-0.5461412829 Li,0,0.7514919886,-1.0910940789,0.6084413758 Li,0,0.2353368235,4.4153638818,-0.8802929334 Li,0,-3.4756244713,1.3738923689,1.4735983381 Li,0,-1.1478438358,-0.2469924072,-1.2542010522 C,0,-1.1720347503,-2.0750634348,0.1782523119 C,0,2.1119074949,0.4616533577,0.5134531627 $\mathrm{Al}, 0,-0.3288571113,2.4597119296,-2.480871247$ 


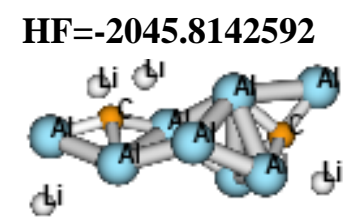

Al,0,-2.4900338013,1.0937641279,0.0519726576 Al,0,-0.2053406345,1.62479678,-1.0197442528 Al,0,-0.6678177218,-1.0841524812,0.5065989029 Al,0,-4.3928469561,-0.7846092395,0.2984481275 Al,0,4.0235700892,-1.2878151901,-0.709131553 Al,0,1.3085640111,-0.5429360932,-1.1418899487 Al,0,2.0674913051,1.9095726793,0.1414424986 Al,0,1.4129117413,-0.247317945,1.7685439067 C,0,-2.5046159153,-0.9552938012,-0.16391895 C,0,2.7718240051,-0.0176979258,0.0985879212 Li,0,-2.6994523247,-2.9015016934,0.0634667977 Li,0,-4.916000392,1.841818433,0.9399890936 Li,0,-1.4979778208,-0.6052858101,-1.9654872813 Li,0,4.0008562155,0.6586410923,1.5423186461

\section{HF $=-2045.8527261$}

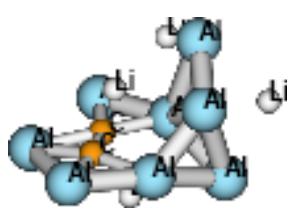

Al,0,1.1067248971,2.7152183179,0.4280211588 Al,0,-2.4230666151,1.1881422742,-1.8288679413 Al,0,3.4329198784,0.6373520678,0.0323322595 Al,0,-2.0644653678,-1.1256209133,-0.9893947756 Al,0,0.0143865917,-1.9809554596,0.3281184278 Al,0,-0.9186211827,1.0052377412,0.5915740117 Al,0,-2.0574477887,-1.1149563453,1.7610595459 Al,0,2.5002737195,-2.2452498936,-0.4827147299 C,0,1.2442406045,0.7732873624,0.2541291649 C,0,1.6242169016,-0.5266289171,-0.004138879 Li,0,-3.953173749,0.2759091083,0.4207507081 Li,0,0.4193123938,0.1263707363,-1.6933503856 Li,0,-1.3778914765,3.3796707966,-0.6409905444 Li,0,0.9484532459,-0.2849946185,2.1063885034

\section{HF $=-\mathbf{2 0 4 5 . 8 6 9 9 8 6 5}$}

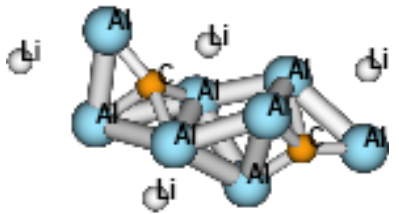

C, $0,-2.5326272108,0.5251533697,0.2500212236$ Al,0,-4.22521076,0.1562559464,-0.5733352444 
Al,0,-1.7772091752,-1.3297996448,-0.6236310844 Al,0,-1.2170166869,1.7349825133,-0.472058459 Al,0,-1.4266896048,-0.0864015219,1.7660865295 Al,0,3.3610607202,-1.697364558,0.660524206 Al,0,0.9826348149,1.1314390592,1.2189839654 Al,0,3.2184067103,0.8331180275,-0.5235825855 Al,0,0.5938264272,-0.365408305,-1.1660278462 C,0,1.894008417,-0.4419349162,0.392178954 Li,0,5.584584652,-0.4833444885,-0.4496637827 Li,0,1.2014186925,2.5573428521,-1.2493410559 Li,0,0.5505590083,-1.7616257659,1.2601021335 Li,0,-3.9351353637,-2.1117027443,-2.0889887362 HF $=-\mathbf{2 0 4 5 . 8 5 3 1 4 1 5}$

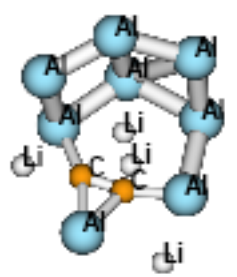

Al,0,0.6290385169,2.1787497454,-1.8261173715 Al,0,-1.9032268177,-0.5392178596,2.3317112075 Al,0,0.3096529035,-1.8286365962,2.4408389975 Al,0,-2.7994665607,1.378947803,0.8687148677 Al,0,3.5748519031,0.6202256507,-0.6737032564 Al,0,-1.6789965719,-1.1458457709,-0.1692614732 Al,0,-1.8765505774,1.1728991209,-1.5447642286 Al,0,0.6667913809,-2.2029336993,-0.1029595984 C,0,1.5294809815,0.5799550987,-1.1769176797 C,0,1.8760856047,-0.5485260653,-0.4896653939 Li, $0,0.3764552627,0.6314058738,0.6081812715$ Li,0,0.2050605137,-0.6207325691,-2.2722573923 Li,0,2.8416295743,-1.5792007767,0.9917384249 Li,0,3.1033133774,3.0908530318,-1.7338191174 HF $=-2045.8497722$

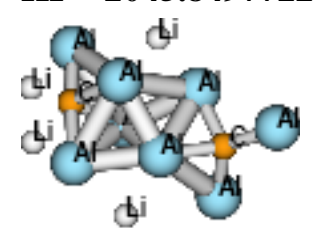

Al,0,-2.5886639986,1.2685520969,-1.3330412684 Al,0,-0.78632291,0.9501213138,0.7593773034 Al,0,-3.9155448255,-0.8934052419,1.0234435472 Al,0,-1.0553072132,-1.481610843,-0.2332291955 Al,0,3.0188004244,-1.6134116112,-0.5367008332 Al,0,0.9094840586,0.0716252643,-1.3184888261 Al,0,1.6371400657,2.011087615,0.6679188066 
Al,0,1.0505120984,-0.7452461563,1.4327189642

C,0,-2.4172245676,-0.0038432877,0.1318576423

C,0,2.4289018226,0.1322745314,0.0770203486

Li,0,3.2969545174,1.437911517,-1.2409747395

Li,0,-0.1019247804,2.6917629241,-1.4867155606

Li,0,0.8023765091,-2.8757904438,-1.5044596145

Li,0,3.4754825447,0.3624996193,1.8124004407

\section{HF $=-\mathbf{2 0 4 5 . 8 7 2 8 6 7 5}$}

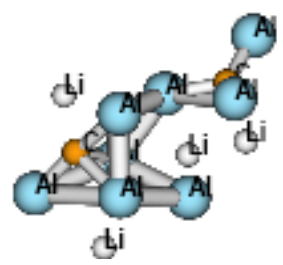

C,0,2.2642189783,-1.0272337746,0.1540413894 Al,0,0.1887016255,2.1800112717,-0.2394765439 Al,0,1.1472211307,-0.0297911333,-1.3792213415 Al,0,0.6639401964,-1.2366796804,1.3482773451 Al,0,-1.1593379995,-0.8545697797,-0.5373618248 Al,0,-2.4933841693,0.4573652721,1.6323251687 Al,0,2.1006537926,0.8702816557,1.0226060301 Al,0,-4.5068190247,-0.7133084272,-0.9236723867 $\mathrm{Al}, 0,3.8891702665,-0.6753092905,-0.8024868525$ C,0,-2.8655465237,-0.0250094863,-0.196866439 Li,0,-2.3978451267,1.8170192103,-1.0043863935 Li,0,-0.0928116632,0.9864025992,2.8005910286 Li,0,1.3723378712,-2.6314607029,-0.7825087758 Li,0,3.0570087974,1.9411925651,-1.4523340026 HF $=-\mathbf{2 0 4 5 . 8 7 0 7 0 1 1}$

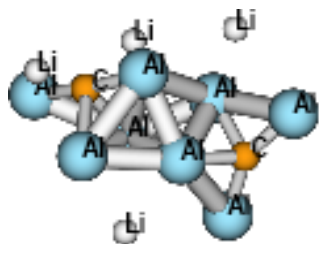

C,0,-2.4474027519,-0.0739370956,-0.2370055013 Al,0,1.5361191393,1.7194574727,1.2409381697 Al,0,-0.983304971,0.7971440355,0.8104209659 Al,0,-0.9660014097,-1.3952575241,-0.8793849202 Al,0,0.7856880928,-1.0015093256,1.4212981947 Al,0,0.9313778887,0.3632534931,-1.1968334277 Al,0,-2.4477740161,1.4667392271,-1.4422300023 Al,0,3.6892844504,0.2287526986,-0.955669386 Al,0,-3.3716168205,-1.5234808695,0.6207276189 C,0,2.2507752022,-0.241745965,0.2994438794 Li,0,3.4883828369,0.0012666682,1.8717623233 
Li,0,-0.0588862452,2.9856042702,-0.8319421001

Li,0,1.6461135253,-2.1075069491,-0.7840428611

Li,0,-1.1020352181,-3.0867611022,1.269187959

HF $=-2045.8718191$

di

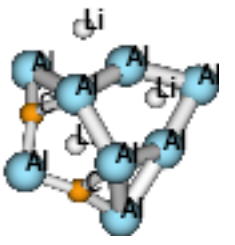

Al,0,2.2979400905,0.5230226571,-0.056336271

$\mathrm{Al}, 0,3.2745743745,-1.47928887,1.1781416399$

Al,0,0.4390006036,0.439066069,-2.0366831688

Al,0,0.7310945902,-1.8613473508,1.2076628465

Al,0,1.1619033696,2.8739093495,-0.819181576

Al,0,-2.2786880765,-0.9784572828,1.9537339754

Al,0,-2.3772912288,1.5296696541,-1.0776408168 Al,0,-1.0997999747,-1.3321400976,-0.609478587

C,0,-2.2818179307,-0.0583693801,0.268752807

Li,0,-4.2624682858,0.3173719533,0.053629749

Li,0,-0.5090031391,-2.0628153244,3.6004090807

Li,0,1.4581734578,-1.8914304036,-1.4347804389

Li,0,-0.520283671,1.0753881996,0.7588715199

C,0,-0.4569810383,1.9578382224,-1.194956854

HF $=-2045.8249972$

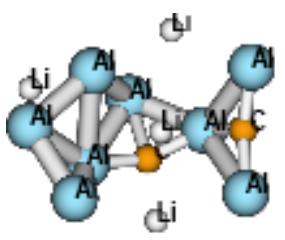

Al,0,-3.4908633407,0.0085357623,-0.9118451225

Al,0,-1.4538866996,-1.6075484214,-1.0265633992

Al,0,-1.6931301023,0.4849848831,1.0522531405

Al,0,-0.1568316457,-1.7181391121,1.4521055258

$\mathrm{Al}, 0,-2.348969038,2.3278368657,-0.6882841474$

Al,0,3.2764884764,-1.2511335997,-1.3809656797

Al,0,2.8421426918,2.2361909398,-0.0442700699

C, $0,0.1739254647,0.3314454436,1.3339986985$

Al,0,1.9261150032,-0.332245586,1.0168706728

C,0,2.9099782085,0.3809019907,-0.483889687

Li,0,0.4095445525,0.4563606994,-0.9183041295

Li,0,0.2303456687,2.4041115536,1.2048069293

Li,0,1.0921339329,-2.8798987815,-0.7452378495

Li,0,-3.1377813285,-2.0486891782,1.0582130385

HF $=-\mathbf{2 0 4 5 . 8 2 5 9 3 5 4}$ 


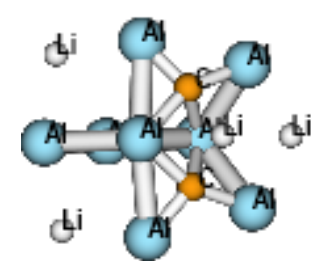

Al,0,1.6586355137,0.0139261719,1.5810263929 Al,0,3.4588554285,0.0258384461,-0.2249042201 $\mathrm{Al}, 0,-2.5692417602,2.0837927881,-0.0240390111$ Al,0,0.478950614,2.8860592272,-0.5712054735 Al,0,-1.0351631621,-0.0082746624,1.1054827348 Al,0,0.5252944294,-2.8774250491,-0.5670015237 Al,0,-2.536880456,-2.1234506552,-0.0259966205 C,0,-0.8145919244,1.4446898732,-0.5680753277 Al,0,0.6346435639,0.0045950272,-0.8347149996 C,0,-0.7922883384,-1.4576888053,-0.5694896484 Li,0,-1.801698078,-0.0133442333,-1.7111996804 Li,0,3.0618719322,-2.5638751188,0.2805431042 Li,0,3.0214588256,2.6103999811,0.2718759982 Li,0,-3.7332802285,-0.0291150377,1.5331056541 HF $=-\mathbf{2 0 4 5 . 8 3 7 7 5 7}$

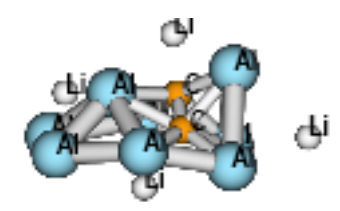

Al,0,-1.5293989347,-2.4264309588,0.6776111473 Al,0,3.0213246791,-0.7886110282,-0.4355502807 Al,0,-1.9631449518,0.4322942145,1.4822266742 Al,0,2.7168122321,1.7620347415,0.2964134486 Al,0,-2.500127879,-0.4119792215,-1.2510609945 Al,0,0.7868932612,-2.2410591424,-0.4861452257 Al,0,0.917833127,0.4011158858,-1.2019011521 Al,0,-0.0408114531,2.1960136973,0.7753392821 C,0,-0.6448287012,-0.6930138512,-0.0058070171 C, $0,-0.9212977704,0.6681614155,-0.4202869008$ Li,0,-3.9739844896,-1.3651253006,1.0250156793 Li,0,-1.4126793702,2.1953968795,-1.5281622319 Li,0,0.7384505881,-0.3170807228,1.6318953331 Li,0,1.6731525307,4.2018751995,0.3433964917 HF $=-2045.8472534$

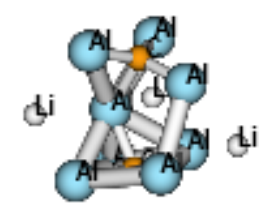

bi 
Al,0,1.1542945321,-0.4043932267,-2.1100694397 Al,0,1.0303577555,-1.9296596098,0.2346933931 Al,0,2.0408808577,0.0815066447,1.8639987458 Al,0,1.9277149991,1.9435600545,-0.3744005107 Al,0,-2.4624031989,1.9166691607,-0.4459266756 Al,0,-0.2161415151,0.6829695474,0.6344107302 Al,0,-1.7064852925,-1.1710470888,-1.4312223964 Al,0,-2.3078585773,-0.8838359295,1.7035402815 C, $0,-2.1355004484,0.0588818758,0.0042203802$ C,0,1.5611585657,-0.0566790412,-0.2300345657 Li,0,3.578480186,-0.3143224499,-0.2392079764 Li,0,0.0605627617,-1.3625551319,2.9237034335 Li,0,0.08836595,-2.8387575377,-2.3158077602 Li,0,-0.2402832281,3.4895613894,-0.2421638811 HF $=-2045.8739472$

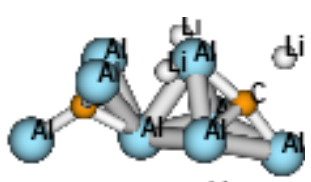

bi

C,0,2.2835814119,0.2451876721,-0.8936954427 $\mathrm{Al}, 0,3.7573654579,0.9445244429,0.194038663$ Al,0,0.5444970386,-0.4204305695,-1.6571383242 Al,0,1.8091745702,-1.0666810057,0.6533119502 Al,0,1.1019079237,1.8567877225,-0.2918298372 Al,0,-3.6504389285,-0.1488657236,2.0805927359 Al,0,-1.7816665044,-2.1187404239,-0.0400762557 Al,0,-2.4141903674,0.7951224505,-0.9958428846 $\mathrm{Al}, 0,-0.4723613396,0.2003573345,1.0167840831$ C,0,-2.3442728292,-0.3387903932,0.6432651034 Li,0,3.1605292932,-0.0428993127,-2.6102964079 Li,0,1.9297460009,1.2072363763,2.3021270517 Li,0,-0.6666792805,1.9224183621,-2.5182298072 Li,0,0.4892061357,-3.0818716369,-0.8320473909 HF $=-2045.8651071$

di

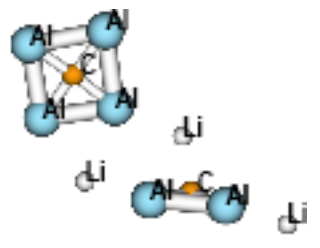

Al,0,-3.9647000568,-1.2501183989,0.

Al,0,-3.2417219453,-3.9644478981,0.

Al,0,-1.1881003585,-0.6710224355,0.

Al,0,-0.5565580846,-3.2782973258,0.

C,0,-2.2514411993,-2.2833959713,0. 
C,0,1.7601282739,2.3239400862,0.

$\mathrm{Al}, 0,2.1417012726,0.8904500565,1.3723398621$ Al,0,2.142535339,3.6429352291,-1.4111006328 Al,0,2.1417012726,0.8904500565,-1.3723398621 Al,0,2.142535339,3.6429352291,1.4111006328

Li,0,-1.3903546616,-5.9133176921,0. Li,0,1.6773400715,-1.5240811638,0. Li,0,2.6418667475,5.9314570983,0. Li,0,-0.2882616778,1.8456873053,0.

\section{$H F=-2045.8561678$}

bi

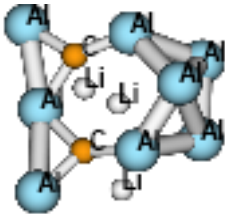

$\mathrm{Al}, 0,2.3042596188,-2.8369327092,-0.6631630786$ Al,0,-2.963968109,-0.9754042369,-1.0845834373 Al,0,2.2314387295,-0.0961595135,-0.432609475 Al,0,-2.5942568191,1.5448556395,-0.7495825818 Al,0,2.6579136092,2.6134885671,0.4588193601 Al,0,-0.8950738593,-2.0541292971,0.3327618447 Al,0,-2.2247065689,-0.159488066,1.4077383525 Al,0,-0.6235763632,1.760297413,1.108712931 C,0,1.0826827674,1.488079736,0.1638766303 Li,0,-0.196020593,0.0230774567,-0.963185588 Li,0,0.0776418165,2.7505260347,-1.2211677532 Li,0,4.4764899083,-1.2307089113,-1.3125578918 Li,0,0.7386926103,-0.3884382695,1.6594671327 C,0,0.9361831791,-1.6244514514,-0.0643580638 HF $=-\mathbf{2 0 4 5 . 8 4 5 7 6 7 8}$

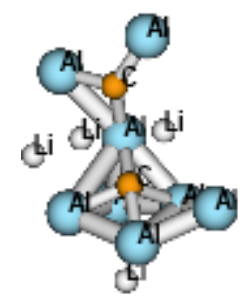

Al,0,2.4624218355,1.2993142797,-0.5097569641 Al,0,1.9089166596,-0.4016601974,1.3964265102 Al,0,0.8095507742,-0.3284296436,-2.1217965202 Al,0,1.080889829,-2.3342705051,-0.4952422014 Al,0,0.9434026155,2.2422233094,-2.4453491373 Al,0,-2.3528385913,-1.0132196293,2.4477333238 Al,0,-3.833848737,1.2080242917,0.5896606233 C,0,0.6938712187,0.4215412146,-0.0941803756 Al,0,-0.8548303279,-0.8035174197,0.0795295082 
C,0,-2.1969011123,0.2956044021,1.0353445452

Li,0,0.2403880454,-2.3485879155,2.3462176702 Li,0,-0.2955384517,0.9376208692,1.8956042469 Li,0,3.4246416329,-1.1339768698,-1.0821794988 Li,0,-1.0726423558,1.6806399116,-0.4538597085 HF $=-2045.8529$

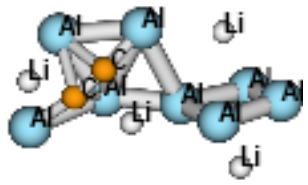

Al,0,-1.5040421301,-0.2111709887,1.183456687 Al,0,3.2336548604,-0.9548034032,1.5274145072 Al,0,-3.8939675019,1.4417210842,0.579337326 Al,0,4.2479518536,0.3405454264,-0.3714154962 Al,0,-3.1203144188,-1.4944913329,-0.5708305303 Al,0,0.9974000225,0.1445698335,0.6944434659 Al,0,2.2493694475,1.6502071111,-1.198704235 Al,0,-0.4689799208,-1.5323171797,-1.358721315 C,0,-2.5053718506,0.7850163619,-0.6016834613 C,0,-1.6150018122,0.0046249945,-1.1858639336 Li,0,-3.9816798641,-0.9233792459,1.9538825034 Li,0,-0.6167914633,1.794337128,-0.9556400343 Li,0,3.0841511122,1.8396885283,1.7737961997 Li,0,2.2104212872,-1.6217248424,-1.2985256541 HF $=-2045.8364099$

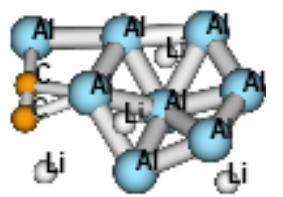

Al,0,0.7342750112,0.0638251763,-0.8681460444 Al,0,-3.4985040751,2.1692406718,-0.1611699011 Al,0,3.2063172229,0.3291726134,-0.0977550363 Al,0,-0.6685419012,2.1252349141,0.0813662766 $\mathrm{Al}, 0,0.1402181983,-2.5603215244,-0.4938200492$ Al,0,2.4910476265,-2.0368051284,0.5027532523 Al,0,-1.3958163074,-0.439603942,0.5868682048 Li,0,0.0437304867,-2.6841289448,2.1031685117 Li,0,1.016862116,0.1297972902,1.8019913054 Li,0,2.7175566761,-1.702688292,-2.0871508865 C,0,-3.4073463045,0.1547544052,0.1286578704 Al,0,1.8517038232,2.4484400498,-0.0371346178 Li,0,-2.6052855689,-2.9251435174,-0.1773863287 C,0,-3.3772680138,-1.1119021394,0.1062796448 HF $=-\mathbf{2 0 4 5 . 8 4 1 9 9 1 6}$ 


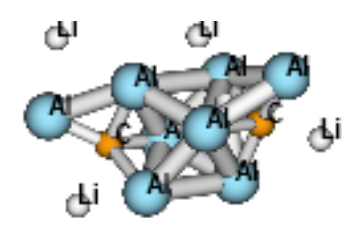

Al,0,-1.1371920462,1.33296401,-0.1093885137

Al,0,-0.8604547128,-1.1570416569,-1.0815132135

Al,0,-0.9049156025,-1.183468317,1.8380949088

Al,0,-3.7969896118,0.6433182193,0.1428486247

Al,0,3.4427418852,1.2787583374,-0.1441255381

Al,0,1.1043403852,0.3885785767,-1.8213588759

Al,0,1.0774158701,0.457814026,1.1474489307

Al,0,1.3339430342,-2.0300324572,0.0880990085

C,0,-2.1808604068,-0.4503945757,0.1568496949

C,0,2.3233482334,-0.3328832036,-0.2544724162

Li,0,3.8335176282,-1.4339438615,-0.9300610112

Li,0,-3.2311305051,-1.9284374441,1.0839653854

Li,0,-3.0704705931,3.2697904765,0.0089529187

Li,0,1.0612546106,2.8252865218,-0.2280682871

\section{HF $=-\mathbf{2 0 4 5 . 8 6 4 9 5 5 5}$}

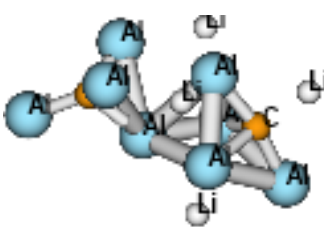

Al,0,-1.3838256301,-0.6465908767,-1.5880715036 Al,0,-0.9796877805,1.5075765821,0.0200668782 Al,0,-3.6005509812,-1.4501479773,-0.0172156356 Al,0,-1.3827390654,-0.687360759,1.572202732 Al,0,1.9342579899,1.2108916392,1.5820268057 Al,0,0.7224253178,-0.8475829242,-0.011148141 $\mathrm{Al}, 0,1.9321543697,1.2513249103,-1.5517481875$ Al,0,4.0870117063,-0.9433656007,-0.0142481405 C,0,-2.4716995411,0.1561701051,0.0031565122 C,0,2.4539854519,0.1268755025,0.000596797 Li,0,-0.3491554641,1.6838275827,2.7256173291 Li,0,-0.3519412832,1.7538927292,-2.6807944718 Li,0,-1.2438325435,-2.9845759996,-0.0379052334 Li,0,-3.7788415457,1.6035361658,0.0208282572

\section{HF $=-\mathbf{2 0 4 5 . 8 6 5 0 3 3 7}$}

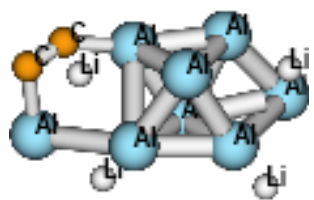

Al,0,-0.3128724623,-1.359769481,1.4948567906 $\mathrm{Al}, 0,-3.4844468205,0.4136180057,-1.0685500458$ 
Al,0,1.0008998814,0.8799217866,1.2657217163

Al,0,-1.7469662164,0.8042283124,1.4601527358 Al,0,-0.6458342324,1.0422225215,-0.9725626221 Al,0,0.8133830212,-1.1424952154,-0.9305127249 Al,0,-1.7128117468,-1.4863368031,-0.8186697697 Al,0,3.5169671208,1.1966070628,-0.2753848635 C,0,2.7693125329,-1.3086098636,-0.8921553874 C,0,3.8039594577,-0.7857981102,-0.3973922179 Li,0,-2.9288659451,2.6917654807,-0.0242184293 Li,0,-3.182401641,-1.4525771582,1.4130905259 Li,0,2.6191839229,-1.3091566332,1.3308201817 Li,0,1.489492654,2.7508007708,-0.8124856732 HF $=-2045.8603999$

C,0,-2.315626905,-0.4702706226,0.0342163169 Al,0,-0.8830057499,0.3634351455,-1.2376622741 Al,0,-3.7441641338,-0.3348761151,-1.2828101278 Al,0,-0.8004062213,-1.2720891025,1.2252103119 Al,0,-1.9224053357,1.3673085057,1.02812655 Al,0,1.1101514142,-1.3178422377,-0.7769223551 Al,0,3.9853969228,-1.1674494461,0.2894119189 Al,0,0.9253819258,0.7284410285,1.0120639898 Al,0,2.5344534051,1.7018873066,-1.0066885892 C,0,2.4579465375,-0.0021532105,-0.0559054148 Li,0,-0.0031294968,2.9481658903,-0.5616509227 Li,0,-1.4073085346,-2.2200375179,-1.1374395558 Li,0,-3.3880820549,-0.8448881931,1.7038567541 Li,0,-0.7095288296,0.763408786,3.2854510816 HF $=-\mathbf{2 0 4 5 . 8 7 2 2 8 2 9}$

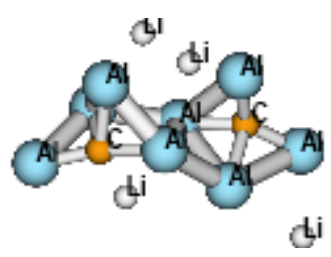

Al,0,1.9639084939,0.2047980248,1.6328572349 Al,0,2.3617262953,1.4183968937,-0.770667017 Al,0,3.8968710675,-1.2627126505,0.2331817293 Al,0,0.5389680476,-0.2795410388,-1.4702859887 Al,0,-1.7813756314,1.8617713555,-0.4157658542 Al,0,-1.7394977439,-1.3998824557,-0.6550886016 Al,0,-0.5648533162,-0.0001017999,1.2518615016 
Al,0,-4.1582719095,-0.4476003922,0.5298362545

C,0,2.2056093839,-0.6008378887,-0.4570184096

C,0,-2.37902704,0.2501392171,0.5011721143

Li,0,0.2794934958,2.483082735,-2.0468711101

Li,0,0.8692054101,2.6416822731,1.1587320345

Li,0,1.024585129,-2.1134974667,0.3801774829

Li,0,-4.0688417035,-2.7220912579,-1.0360392715

\section{HF $=-2045.8574704$}

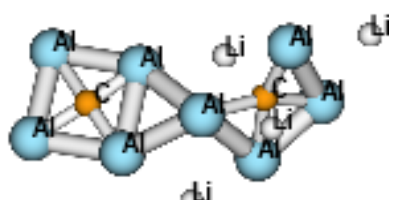

bi

C,0,-2.4382825358,0.0173127924,0.0274398742

Al,0,-3.4363596053,-1.5697788944,0.6196703285 Al,0,-0.5904952572,0.2583368474,1.0205759898

Al,0,-1.9653501237,1.9473399103,-0.5261920142 Al,0,-4.0092871032,0.4454630775,-1.1720580785 Al,0,3.956905199,-1.8763362679,-0.7704279536 Al,0,2.0093777609,1.2899769409,0.6059178805 Al,0,1.3106808678,-1.2091733878,-0.3584906457 Al,0,4.7368791567,0.6371238784,0.2873641843 C,0,2.9691343871,-0.2785246369,-0.0175881456 Li,0,-5.7610216121,-1.5478888101,-0.8118121277 Li,0,-0.0166869533,2.8983016024,1.1592616944 Li,0,-1.2018834348,-1.2291023677,-1.1706466394 Li,0,-2.8022989137,0.7349874788,2.0759349542

\section{HF $=-2045.8425784$}

का

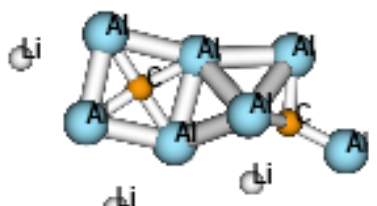

di

C,0,-0.757924435,-2.4337892409,0.

Al,0,1.2591004209,-2.6062089569,0.

Al,0,-0.4796750124,-1.2325359843,1.6780781352

$\mathrm{Al}, 0,-1.6911397928,-4.1231701543,0$.

Al,0,-0.4796750124,-1.2325359843,-1.6780781352

Al,0,1.3223293903,0.2601240907,0.

Al,0,-0.5197407843,3.701035132,0.

C,0,0.4730837759,2.0444708328,0.

Al,0,2.1318582877,3.0176821616,0.

Al,0,-1.2331394276,1.0420701245,0.

Li,0,3.4426007214,-1.4305681023,0.

Li,0,-2.5485020807,-1.2265585731,0. 
Li,0,-3.1587437033,2.9209793175,0.

$\mathrm{Li}, 0,1.4913480798,5.6001223145,0$.

\section{HF $=-\mathbf{2 0 4 5 . 8 2 6 1 7 9 5}$}

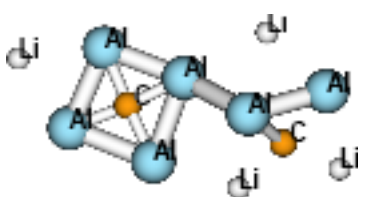

Al,0,1.3302612577,-1.3323962649,3.2138262143

Al,0,-0.4625274605,-3.3850236766,2.7311403836 Al,0,0.6334478822,-0.0796539637,0.9755181404 Al,0,-1.2319814016,-2.0954004724,0.3532162358 C,0,0.0247121528,-1.754184206,1.7892628726 C,0,-0.8567445757,1.0350087247,-2.2467423162 Al,0,0.8139823624,0.0533615347,-1.7159469031 Al,0,-0.5713063276,3.4454459109,-1.7746820372 Al,0,-1.175574453,1.4602508348,-0.3091613153 Al,0,1.1815411391,2.2059425569,-3.0140940182 Li,0,1.1017749051,-3.3955859263,4.9540294834 Li,0,-1.8663541429,-0.6666497519,-1.9728061093 Li,0,1.6381030482,2.4726037199,-0.1560867804 Li,0,-1.4534452923,1.8470349287,-3.9027167415

\section{HF $=-\mathbf{2 0 4 5 . 8 4 0 9 8 7 2}$}

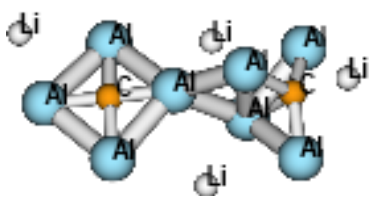

Al,0,4.4354716988,0.3831309232,0.0042164501 Al,0,2.6603177229,-1.7561168681,-0.1972890576 Al,0,2.2821980366,2.1087643497,-0.0360299439 Al,0,0.5837537132,-0.0638091682,0.2909471053 C,0,2.5020132256,0.1679544473,0.1438738917 C,0,-3.0023750089,-0.0979313852,0.3257741961 Al,0,-1.5419598576,0.0708920344,-1.2188196363 Al,0,-1.6578554859,-0.1518273935,1.8013781388 Al,0,-3.3273817774,1.8121204429,-0.1872469644 Al,0,-3.1662475123,-1.94481622,-0.4326972255 Li,0,5.389219731,-2.0964013188,-0.4723211034 Li,0,-0.5098958113,2.5543835418,-0.7735282591 Li,0,-0.329370488,-2.3609429169,-1.0896437379 Li,0,-4.7118481981,-0.2232171991,1.2902085034 HF $=-\mathbf{2 0 4 5 . 8 5 8 4 8 5 4}$ 


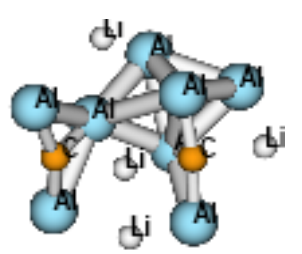

Al,0,-1.1418958178,-2.0526798459,-1.2258932617 Al,0,-3.2955660999,-0.5969582822,-0.3529357061 Al,0,-1.1444279031,0.7959843853,-0.9973010092 Al,0,-1.1399650728,-1.020796868,1.2903935536 Al,0,1.0394634046,-0.6829639679,-0.8133699646 Al,0,3.0971261636,-1.6468162815,0.9350721529 Al,0,-0.7286529338,2.7705224094,0.8282205072 Al,0,2.7639742111,1.6445588777,-0.8370779357 C,0,2.6187370295,0.0023019201,0.1161149462 C,0,-1.0580525005,0.8972479292,1.1749844883 Li,0,-3.0364891512,1.0487448722,1.7317859741 Li,0,1.0853933863,0.6581622862,1.5427238368 Li,0,0.6643451528,-3.1239644334,0.8233332815 Li,0,0.5484724291,3.037605726,-1.5975114193 $H F=-2045.8647263$

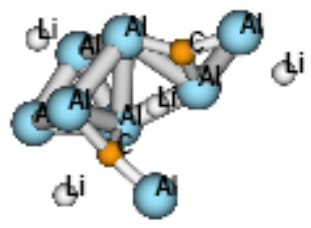

Al,0,1.256218802,-2.2894835012,-1.0683970742 Al,0,3.17303886,-0.5283956729,-0.5272134782 Al,0,-1.9277089835,0.1323698817,-1.1881226944 Al,0,-0.5784043785,-1.5965466855,0.8288313002 Al,0,0.7457770322,0.3662110473,-1.0566293965 Al,0,1.6817812362,0.1925669576,1.7251313051 Al,0,-3.9993410949,-0.3828750073,0.6494100888 Al,0,0.256911821,3.2754002721,-0.0816348384 C,0,1.0943113566,1.6335963629,0.4645347712 C,0,-2.1186832856,-0.4887612855,0.6887129241 Li,0,-4.5096024243,0.5161190815,-1.8905413475 Li,0,-0.9434863821,1.076447885,1.4290615233 Li,0,2.9663075044,2.0826271864,-0.2907583809 Li,0,1.8996742163,-2.3649359056,1.5597835613 HF $=-2045.8410709$

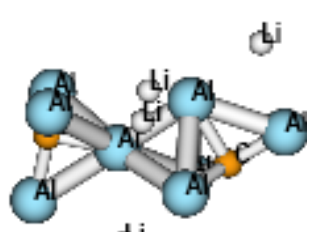

di 
Al,0,-1.2396875512,-1.0507819978,-1.6854828896 Al,0,-1.4276264887,-0.5481291652,1.1600306685 Al,0,-4.1473549616,-0.4182849693,0.2640031453 Al,0,-1.4523778137,1.7227166106,-0.636205774 Al,0,2.732135318,-1.8175944884,0.5437869184 Al,0,2.4890175394,1.3528616264,1.7430803076 Al,0,0.6755224728,0.1049273752,-0.140675629 Al,0,3.044134249,0.8191080861,-1.5478415352 C,0,-2.5148661278,0.0293312917,-0.5872008216 C,0,2.6744310163,0.1118226088,0.2465046828 Li,0,-0.3056691387,2.0235626925,1.9265314995 Li,0,0.0646994686,-2.8080223723,0.0989149743 Li,0,-3.4349754284,-1.2991996984,2.7367943508 Li,0,0.4371766775,1.087118241,-2.7838611314 HF $=-\mathbf{2 0 4 5 . 8 4 2 3 4 6 4}$

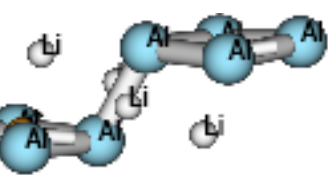

Al,0,2.849561448,-1.9889326419,-0.0903874969 Al,0,1.0408727893,-0.0039431802,-0.891139998 Al,0,4.7532464548,-0.0040016692,0.4655281606 $\mathrm{Al}, 0,2.8525973441,1.9813123879,-0.0991565169$ Al,0,-2.8538678623,-1.9811155334,0.1029540039 Al,0,-2.8491397444,1.9887994712,0.0918111838 Al,0,-1.0401904717,0.0041047952,0.8884796205 Al,0,-4.7531554635,0.0048805268,-0.4670390244 C,0,2.7748956019,-0.0035464138,0.0080385401 C,0,-2.7743969472,0.0032749705,-0.0110160697 Li,0,0.0033646687,2.615210968,-0.0046288646 Li,0,-0.0029197831,-2.6151996744,0.0088698438 Li,0,-1.5359141672,-0.004341349,-1.7756100111 Li,0,1.534799163,0.0000882648,1.7727743828

\section{HF $=-\mathbf{2 0 4 5 . 8 7 8 1 2 4 5}$}

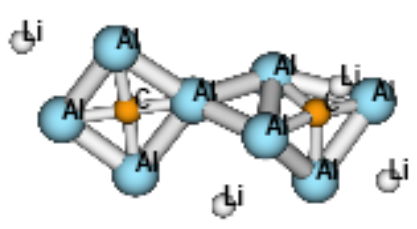

C,0,-2.6651981447, 0.1243906244,0.2685694868 Al,0,-3.3683650692,-1.1134259369,-1.0777545419 Al,0,-0.8687520815,-0.2164807913,-0.3540810596 Al,0,-2.0706761258,1.4890414321,1.5425067342 Al,0,-4.477910538,0.472749064,0.9344478597 Al,0,4.3314649667,-1.2675526125,-0.1929094028 Al,0,1.2847565549,1.0110292828,-1.3015723779 
Al,0,1.3900958149,-1.6435768391,0.0040502574

Al,0,3.0801970092,1.1264734671,0.6978616293

C,0,2.7146570208,-0.309780352,-0.7123063552

Li,0,-6.0502176314,-1.0499074418,-0.6508743772

Li,0,0.5492828148,2.2446327927,1.1777550757

Li,0,3.1236562263,-0.3441572729,-2.6665792742

Li,0,5.3081818699,0.1344307571,1.9327928868

HF $=-2045.8534914$

di

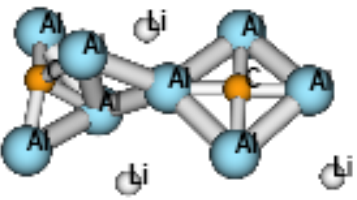

Al,0,-0.0304271716,-2.8352442691,3.4321887098

Al,0,-0.4375327717,-3.0886182803,0.6862302088 Al,0,0.6066876592,-0.1792183896,3.0423046885

Al,0,-0.3153364974,-0.3798368778,0.4309640269 C,0,-0.1668792863,-1.5981535075,1.930460521

C,0,-0.2422680668,2.1075824072,-2.1516433055 Al,0,1.2312346159,0.8295746924,-1.289963175 Al,0,-1.7062477029,1.489218679,-0.9423309161 Al,0,0.8421327441,3.4987100755,-1.1998244688 Al,0,-0.0819443341,0.7933554623,-3.6550435022 Li,0,-0.3685855536,-5.2882918825,2.3583415052 Li,0,1.5359163036,1.8917501792,1.2021257644 Li,0,0.3229912826,-1.6254343848,-2.0306237606 Li,0,-1.1424823397,3.4487068886,-3.2737554186 HF $=-\mathbf{2 0 4 5 . 8 5 8 4 8 4 5}$

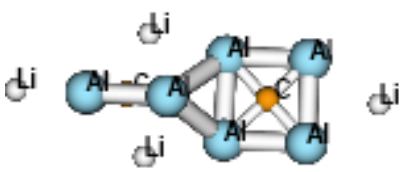

Al,0,3.8080372911,1.2172826079,0. Al,0,2.099533037,3.4022477743,0. Al,0,1.6424691187,-0.4691365489,0. $\mathrm{Al}, 0,-0.0593418932,1.706601584,0$. C,0,1.8872190057,1.4758589036,0. C,0,-1.7694324906,-1.3842595274,0. Al,0,-0.6992186642,-0.5471528313,1.5137885779 Al,0,-2.8037644807,-2.1919049917,-1.4532049845 Al,0,-0.6992186642,-0.5471528313,-1.5137885779 Al,0,-2.8037644807,-2.1919049917,1.4532049845 Li,0,4.7866142583,3.7426587623,0. Li,0,0.0224817274,-2.5828205754,0. 
Li,0,-4.6439394363,-3.6287660247,0.

$\mathrm{Li}, 0,-2.501231723,0.6439167436,0$.

\section{HF $=-\mathbf{2 0 4 5 . 8 6 2 3 9 8}$}

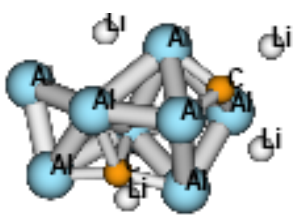

Al,0,3.0200658856,0.1687176784,-1.532303551 Al,0,1.6060307274,-0.0714972323,0.8039756566 Al,0,-0.0952851747,-2.1835290235,-0.1201772467 Al,0,-0.5743206981,-0.7578071866,2.1888014568 Al,0,1.9379025145,2.2888657958,-0.4174653923 Al,0,-1.6747716285,1.4929916801,0.5012090878 Al,0,-2.5158060188,-0.707045013,-0.9370996131 Al,0,0.0019333667,0.5798959309,-1.4331738532 C,0,0.283011478,1.5630889791,0.3620417639 C,0,-1.7114902573,-1.6279995012,0.8090064232 Li,0,-2.8768829009,-3.0486591674,0.0555445534 Li,0,-3.1441083642,-0.4538572199,1.7207025213 Li,0,0.2336974597,1.7853316134,2.3847248661 Li,0,1.2526724761,-1.6655622445,-2.4027233424

\section{HF $=-\mathbf{2 0 4 5 . 8 6 0 7 7 6 8}$}

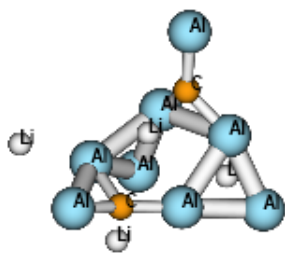

Al,0,-0.7097199792,0.3820582592,2.7113426126 Al,0,1.7475959605,3.0966076374,-0.7516564926 Al,0,-1.9095849344,-0.9293550511,0.6828349943 Al,0,-0.3728887994,1.6352948296,-0.6657061717 Al,0,0.8442238233,-1.0519092539,1.2995544417 Al,0,2.1039772224,0.5436869819,-0.5751037644 Al,0,-3.3285179697,-0.3468352365,-1.6989162364 Al,0,2.8020644107,-2.9125404847,-0.7452894111 C,0,1.7518048782,-1.350005541,-0.3528271435 C, $0,-1.975820844,0.5660745056,-0.7068011138$ Li,0,-3.580438091,-2.7867325893,-0.4166406099 Li,0,-0.0558474241,-1.0318990609,-1.4817653069 Li,0,1.5140904949,1.9352212077,1.9254716046 Li,0,-2.5307552295,1.6442392245,0.9782642797 HF $=-\mathbf{2 0 4 5 . 8 3 9 7 9 3}$ 


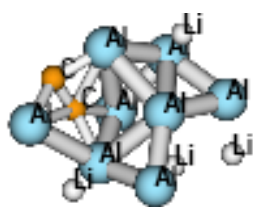

Al,0,0.2218207879,-1.0197876843,1.7496391134 Al,0,3.3566723745,0.4862486175,0.0024121655 Al,0,-0.2449443598,-2.0223922796,-1.0574932369 $\mathrm{Al}, 0,2.1463316916,-1.754714963,0.2546728757$ Al,0,0.8233635197,0.713506679,-0.8206032105 Al,0,-1.3333862106,1.0032484944,0.9951026245 Al,0,-0.3663476893,2.9900830617,-0.3706990152 $\mathrm{Al}, 0,-3.5324645155,-0.4068462173,-0.3815257311$ C,0,-1.5549463209,-0.9237004502,0.4697494378 C,0,-2.1124668503,-1.817350593,-0.3529418255 Li,0,2.4370283948,2.8750843883,-0.8621247414 Li,0,1.3945309427,1.4110970049,1.8894858113 Li,0,1.9803264027,-1.1277203512,-2.387981337 Li,0,-3.1182569909,2.3698096416,-0.4828524944 HF $=-\mathbf{2 0 4 5 . 8 2 7 8 2 3}$

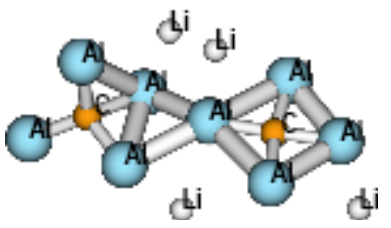

C,0,-2.551099132,-0.088708762,-0.037945152 Al,0,-2.8035676663,1.8325851595,-0.0142157169 Al,0,-0.5939268182,-0.0392156268,0.1607452913 Al,0,-2.5993472571,-2.0480217681,0.0585871994 Al,0,-4.512148449,-0.2574546294,-0.2628563931 Al,0,3.2885726751,-0.4273444976,1.7277058929 Al,0,1.6638302519,-1.2970437221,-0.4607119379 Al,0,1.7210247609,1.4517264161,-0.5390537325 Al,0,4.8010051146,0.6362045142,-0.914788531 C,0,3.092125642,0.1015940615,-0.1572833121 Li,0,-5.5680097615,2.1839150549,-0.2885392848 Li,0,-0.1541583973,-3.1128229218,-0.2981049288 Li,0,0.8070547774,-1.4900907728,2.3516612097 Li,0,-0.3505242902,3.0370060425,-0.3146790478

\section{HF $=-\mathbf{2 0 4 5 . 8 4 7 7 3 5 7}$}

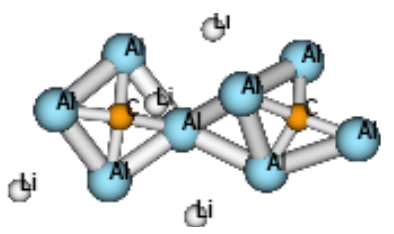

C,0,-2.4047625721,0.1587499605,0.1919954772 Al,0,-4.2940976146,0.4373573101,0.6150914456 
Al,0,-0.5715578681,-0.2683479582,-0.5291079605

Al,0,-2.2179885473,2.0332510517,-0.4758468613 Al,0,-2.8146010719,-1.725709935,-0.3490291853 Al,0,1.9395676113,-1.6178572274,-0.3730701525 Al,0,3.1320052729,1.6364809234,-0.7507277967 Al,0,1.3071045411,0.6764766353,1.0421518764 Al,0,4.7542427561,-0.8235118163,0.5704314886 C,0,2.9887627112,-0.0675564495,0.2406550053 Li,0,-5.5129976207,-1.9612601056,0.1995450616 Li,0,0.4981946113,2.7294783015,-0.804184475 Li,0,-1.2378179707,0.4525957774,1.9274712752 Li,0,-0.2656379761,-2.9118032569,-1.1043351958 HF $=-\mathbf{2 0 4 5 . 8 6 1 1 7 1 6}$

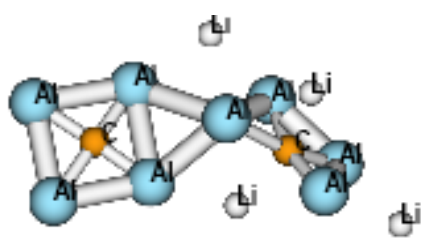

C,0,2.4364268587,-0.0010044843,0.1008670994 Al,0,4.0480105436,0.0127491698,-1.1200827428 Al,0,2.0208624592,1.6681343796,-1.0369330534 Al,0,0.5678887579,0.5545074412,0.9106161819 Al,0,3.3802796355,-1.3387143774,1.1894041273 Al,0,-1.3249109079,-1.2468478761,-0.0092791959 Al,0,-1.9963544116,1.4364879508,0.1094238558 Al,0,-3.9729029771,-1.9772233748,-0.2819101386 Al,0,-4.7266932396,0.7495721753,-0.0828176993 C, $0,-2.9717704999,-0.2368785145,-0.0285969166$ Li,0,5.7411584472,-1.796527148,-0.1032162851 Li,0,0.0457939212,3.1145864727,0.2107920432 Li,0,2.758794192,1.3127936306,1.836449217 Li,0,1.2081613289,-1.5426374075,-0.6950577924 HF $=-2045.8425779$

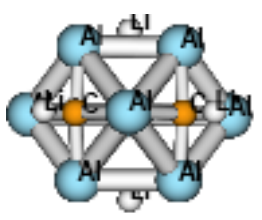

Al,0,-1.456356507,1.9970209226,0.1979402613 Al,0,0.0011398628,0.0010212297,1.8810450571 Al,0,-1.473520812,-1.9896181096,0.1909318454 Al,0,-2.8826501168,0.0147639359,-1.1368683509 Al,0,2.8914130198,-0.0109443821,-1.1289376699 Al,0,-0.0006542299,-0.0013389585,-1.0012187191 $\mathrm{Al}, 0,1.4697127437,1.9899898528,0.182605316$ 
Al,0,1.4566762393,-1.9978735304,0.1903776287

C,0,1.567724762,-0.003454494,0.5418513389

C,0,-1.5685024399,0.0032328774,0.5433794621

Li,0,-2.4989371094,0.0000189376,2.3121565464

Li,0,-0.0125279521,-2.539315501,-2.0421130595

Li,0,2.5004218874,-0.0081380087,2.3100237838

Li,0,-0.0123623363,2.5347869766,-2.0459888037

HF $=-2045.872921$

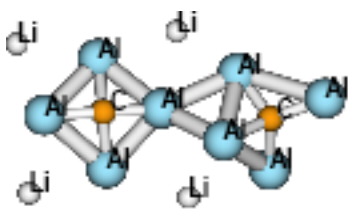

C,0,-3.1238734581,0.1732511596,0.2176668832

Al,0,-4.9574259162,-0.4611619991,0.4112808846 Al,0,-1.5469595034,0.9326802449,1.221753854

Al,0,-2.0810203396,-1.4311475744,-0.2015775797 Al,0,-3.0360125135,1.8081063996,-0.9077923599 $\mathrm{Al}, 0,2.3366633815,1.6658978245,-0.3494653409$ Al,0,2.5265861721,-2.0216915708,-0.4289074575 Al,0,4.3528307996,-0.1340960609,0.3502436981 Al,0,0.4739880526,-0.2537846886,0.2057575172 C,0,2.4194725001,-0.1935423625,0.3212846546 Li,0,-0.0636851653,-2.9348249563,-0.9446805801 Li,0,4.9495311302,2.4478475428,-0.3406766562 Li,0,5.2207484701,-2.6141637138,-0.4867349987 Li,0,-0.3286097631,2.6875790409,-0.6114147759 HF $=-2045.8330693$

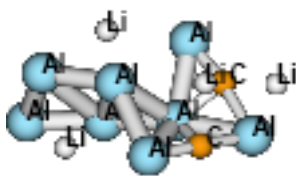

Al,0,3.212525083,-0.5179641688,1.2054765134 Al,0,3.9336394581,-0.803586498,-1.2150383835 Al,0,-0.2848519654,2.6723235671,0.1269589388 Al,0,1.3807996203,-0.6137100481,-1.1728687563 Al,0,0.7763321357,0.2912152395,1.3523820262 Al,0,-1.5981392683,-2.2165170993,0.7913693291 Al,0,-3.5851797002,0.9310823516,-0.7794068439 C,0,-1.9180715521,1.8500063163,-0.2684357809 $\mathrm{Al}, 0,-1.0444215862,0.0712836828,-0.7136301697$ C,0,-2.661745098,-0.8160621887,0.0965396555 Li,0,-2.2305892367,0.7023747443,1.5224458063 Li,0,2.4813490599,2.1046209351,0.1460147048 
Li,0,1.141497694,-2.5563998026,1.1434034749

Li,0,-4.3256739181,-1.5130345815,-0.7141232361

\section{HF $=-2045.8206314$}

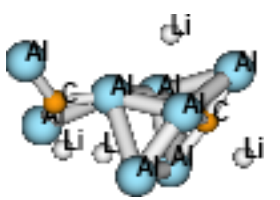

Al,0,3.0268741165,0.2327066425,-2.2542906994 Al,0,0.4945204275,1.4034884704,-1.3237330029 $\mathrm{Al}, 0,1.6101715002,1.312844996,1.2446168716$ Al,0,1.7449980201,-0.9890078568,-0.0128265185 Al,0,-1.6467115464,-1.795219599,2.1819049491 Al,0,-3.8254976339,-2.0836712349,-0.4529114073 Al,0,-0.8546513871,-0.590716809,-0.3259222614 $\mathrm{Al}, 0,-0.8132437649,2.273153912,0.8568048469$ C,0,2.4137876402,0.8890295887,-0.5437498153 C,0,-2.4663693545,-1.1792942777,0.5754607783 Li,0,0.9411767049,-0.9766462937,2.6897629573 Li,0,0.6657267581,-1.0558434891,-2.7524245341 Li,0,2.6915709983,2.8771144439,-0.6893737451 $\mathrm{Li}, 0,-3.0513032043,0.7603977924,1.062828024$ HF $=-2045.872275$ 


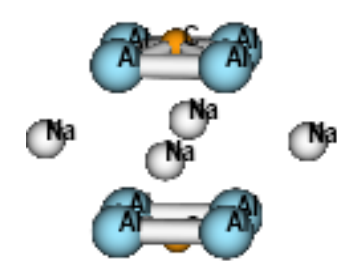

Al,0,1.9325658797,0.,-2.1508375707

Al,0,0.,-1.9325658797,-2.1508375707

Al,0,-1.9325658797,0.,-2.1508375707

Al,0,0.,1.9325658797,-2.1508375707

Al,0,0.,1.9325658797,2.1508375707

Al,0,-1.9325658797,0.,2.1508375707

Al,0,0.,-1.9325658797,2.1508375707

Al,0,1.9325658797,0.,2.1508375707

C,0,0.,0.,2.4924749982

C,0,0.,0.,-2.4924749982

$\mathrm{Na}, 0,2.3437836706,2.3437836706,0$.

$\mathrm{Na}, 0,-2.3437836706,-2.3437836706,0$.

$\mathrm{Na}, 0,-2.3437836706,2.3437836706,0$.

$\mathrm{Na}, 0,2.3437836706,-2.3437836706,0$.

\section{HF $=-\mathbf{2 6 6 4 . 9 6 2 1 4 8 1}$}

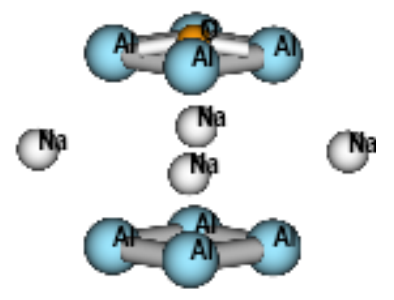

Al,0,1.9336048716,0.,-2.3597979873 Al,0,0.,-1.9336048716,-2.3597979873 Al,0,-1.9336048716,0.,-2.3597979873 Al,0,0.,1.9336048716,-2.3597979873 Al,0,0.,1.9336048716,2.3597979873 Al,0,-1.9336048716,0.,2.3597979873 Al,0,0.,-1.9336048716,2.3597979873 Al,0,1.9336048716,0.,2.3597979873 C,0,0.,0.,2.662699451 C,0,0.,0.,-2.662699451 $\mathrm{Na}, 0,3.7212648066,0 ., 0$. $\mathrm{Na}, 0,-3.7212648066,0 ., 0$. $\mathrm{Na}, 0,0 ., 3.7212648066,0$. $\mathrm{Na}, 0,0 .,-3.7212648066,0$.

\section{HF $=-\mathbf{2 6 6 4 . 9 3 5 3 4 0 8}$}

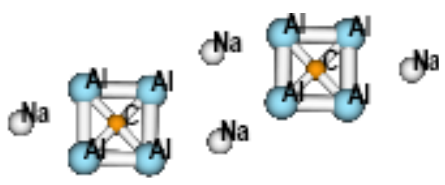


$\mathrm{Na}, 0,0.0294791441,1.8701968044,0.0036781917$

$\mathrm{Na}, 0,-0.0295239542,-1.8700204604,-0.006052047$

Na,0,-8.1641241441,-0.2794279215,-0.1398764725

$\mathrm{Na}, 0,8.1641032134,0.2796705772,0.143627708$

Al,0,-5.3783966916,0.8813276281,-0.090845912

Al,0,-3.0329383124,-2.2739251275,-0.0577079348

Al,0,-5.6898662681,-1.981141742,-0.1017250315

Al,0,-2.6702143747,0.4977250215,-0.0461010881

Al,0,3.032822726,2.2737530485,0.057763766

Al,0,5.3785422249,-0.8812974125,0.0902595125

Al,0,5.6897259835,1.9811778561,0.10437506

Al,0,2.6703782502,-0.497942837,0.0429864213

C,0,-4.2039523157,-0.6886149347,-0.0742577823

C,0,4.203956842,0.6885478253,0.0738888667

HF $=-2664.951836$

Na

(A) $A$

dja

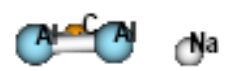

Al,0,-4.5624338723,-2.0748779039,0.

Al,0,-2.6107191623,-4.19150544,0.

$\mathrm{Al}, 0,-2.6006318501,-0.2620590541,0$.

$\mathrm{Al}, 0,-0.6582432717,-2.2663884938,0$.

C,0,-2.599435741,-2.2324492423,0.

C,0,2.4938591157,2.1049890508,0.

Al,0,2.9898466785,0.7638855202,1.4082516994

Al,0,2.5412681452,3.4497366443,-1.4465641815

Al,0,2.9898466785,0.7638855202,-1.4082516994

$\mathrm{Al}, 0,2.5412681452,3.4497366443,1.4465641815$

$\mathrm{Na}, 0,-5.5409544043,-4.9121101535,0$.

$\mathrm{Na}, 0,2.3588821217,-1.9712578517,0$.

$\mathrm{Na}, 0,2.4987938136,6.1053939074,0$.

$\mathrm{Na}, 0,-0.0039178612,1.2819183217,0$.

HF $=-\mathbf{2 6 6 4 . 9 4 6 5 9 0 6}$

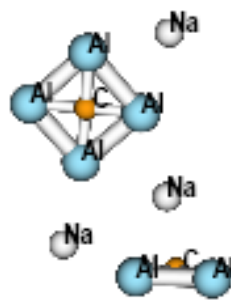

Al,0,-4.5433776439,-2.7611909666,0.

$\mathrm{Al}, 0,-2.3850348633,-4.4801693342,0$. 
Al,0,-2.7651415891,-0.5355142789,0.

$\mathrm{Al}, 0,-0.6760320156,-2.2901642526,0$.

C,0,-2.6063177807,-2.478857666,0.

C, $0,2.5014757718,1.7144402912,0$.

Al,0,2.99698579,0.3668917582,1.4054971929

Al,0,2.5651476135,3.056744936,-1.4471005062

$\mathrm{Al}, 0,2.99698579,0.3668917582,-1.4054971929$

Al,0,2.5651476135,3.056744936,1.4471005062

$\mathrm{Na}, 0,-5.7809632172,-0.0465810675,0$.

$\mathrm{Na}, 0,2.3520613993,-2.3745285074,0$.

$\mathrm{Na}, 0,0 ., 0.9313652379,0$.

$\mathrm{Na}, 0,2.5941930015,5.7118766115,0$.

HF $=-2664.9427294$

(1) $A=A=A$

$(A-A)=A$ A

Al,0,2.9894348344,-1.9795680272,-0.1528371953

Al,0,1.0999641933,-0.0032427811,-0.8926870604 Al,0,4.8114922659,-0.0046464443,0.5175096608 Al,0,2.9949093035,1.9717228381,-0.1637894322 Al,0,-2.9948095394,-1.9716802257,0.1640532789 Al,0,-2.9895313201,1.9796228491,0.1525927979 Al,0,-1.0999844408,0.0035760627,0.8927898815 $\mathrm{Al}, 0,-4.8114466486,0.0044901793,-0.5176247953$ C,0,2.8907057082,-0.0036446344,-0.1089734257 C,0,-2.890724796,0.0036695312,0.10910378 $\mathrm{Na}, 0,0.0036894418,2.9979862832,-0.0087601329$ $\mathrm{Na}, 0,-0.0036935053,-2.9978621335,0.0086045484$ $\mathrm{Na}, 0,-1.6855275685,-0.0060693833,-2.1890719127$ $\mathrm{Na}, 0,1.6855081866,0.0056073025,2.1891479615$ HF $=-\mathbf{2 6 6 4 . 9 5 5 3 1 9 1}$

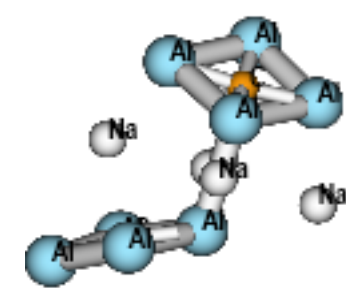

Al,0,4.3872894956,-0.9842015224,0.0109780979 Al,0,3.4141369522,1.5927374542,0.1726752661 Al,0,2.0254314758,-1.8493323216,-1.1806519212 Al,0,1.0783843826,0.8401397187,-1.2025233248 Al,0,-3.6721966778,0.5190831752,-0.6846750593 Al,0,-4.0404269683,-1.8528766717,0.6761638888 Al,0,-1.6135186587,-1.522923663,1.9986235791 
Al,0,-1.3059730276,1.0224419645,0.7508484493

C,0,2.6150231827,-0.1080203455,-0.4548715102

C,0,-2.5429034578,-0.482804877,0.5983031746

$\mathrm{Na}, 0,1.3705091602,-0.6833464958,1.8476766594$

$\mathrm{Na}, 0,-1.5366838517,2.1800787242,-2.1534691258$

$\mathrm{Na}, 0,-1.0749900309,-1.7360793193,-1.2609516524$

$\mathrm{Na}, 0,0.8790402672,3.2028985086,0.8486262394$

$\mathbf{H F}=-\mathbf{2 6 6 4 . 9 4 8 2 9 7 8}$

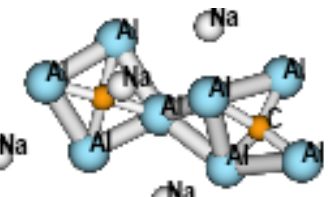

C,0,-2.4468436163,0.1485553785,0.1309656023

Al,0,-4.3089132943,0.4195160958,0.7162147621

Al,0,-0.5579392652,-0.1846169113,-0.452698325

$\mathrm{Al}, 0,-2.4111435539,2.0405253726,-0.5256369317$

Al,0,-2.8295691771,-1.7259777325,-0.4135018881

Al,0,1.9370313884,-1.5720604174,-0.356970926

Al,0,3.4253957754,1.6031036194,-0.676703083

Al,0,1.40309111,0.7195533798,1.0662736547

Al,0,4.7709441078,-0.951363724,0.6551852005

C,0,3.0662357355,-0.0818428779,0.2883186187

$\mathrm{Na}, 0,-5.7421654567,-2.2221015686,0.3263579862$

Na, $0,0.5663776497,2.8999230447,-1.1353673167$

$\mathrm{Na}, 0,-1.311709614,0.7829385084,2.3410927915$

$\mathrm{Na}, 0,-0.2603755271,-3.1278089565,-1.5624901428$

$\mathrm{HF}=-\mathbf{2 6 6 4 . 9 4 2 9 4 8}$

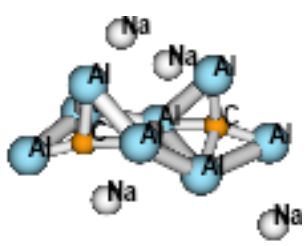

Al,0,2.1073573222,0.1507436751,1.7264764689

Al,0,2.4954990959,1.4417943399,-0.7071771246

Al,0,4.0831935955,-1.244464271,0.2750633508

Al,0,0.6909532344,-0.3773891477,-1.3385707141

Al,0,-1.8396211567,1.9515432998,-0.1090018137

Al,0,-1.6512172163,-1.3908405542,-0.5939418733

Al,0,-0.434300833,-0.0203768478,1.3111468859

Al,0,-4.1264969597,-0.3209004577,0.6018453171

C,0,2.3878947751,-0.5861453225,-0.3822095487

C,0,-2.2696763062,0.1833327418,0.6005761735

$\mathrm{Na}, 0,0.2362702764,2.5038153605,-2.396805945$

$\mathrm{Na}, 0,1.0537823525,2.9912465498,1.4738634356$ 
Na,0,1.1893985855,-2.6179740833,0.6761978918

$\mathrm{Na}, 0,-4.110276931,-2.8820473713,-1.2501759469$

\section{$\mathrm{HF}=-\mathbf{2 6 6 4 . 9 3 5 4 6 2 9}$}

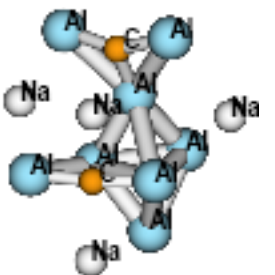

Al,0,-1.0737220244,-2.021012229,-1.4188315054 Al,0,-3.367476887,-0.7855203741,-0.6060865515 Al,0,-1.2522442845,0.7393762382,-1.0691858536 Al,0,-1.1821025403,-1.0780528482,1.1399184548 Al,0,1.05937447,-0.5640471376,-0.8664813314 Al,0,3.334522206,-1.8005196012,0.6185793171 Al,0,-0.8590107698,2.7382724762,0.7980707484 Al,0,3.1914479913,1.4590539504,-1.0810610919 C,0,2.8086342084,-0.1345447902,-0.1221973839 C,0,-1.1047701347,0.8409979949,1.063951417 $\mathrm{Na}, 0,-3.4816607615,1.012316889,1.7611721572$ $\mathrm{Na}, 0,1.3632995791,0.6948946283,1.8706258481$ $\mathrm{Na}, 0,0.7130401895,-3.50767668,0.7837301553$ $\mathrm{Na}, 0,0.6522818531,2.9662037629,-1.9923020355$ $H F=-2664.9370146$

Al,0,1.4168996184,0.2943499238,2.0134801017 Al,0,-0.0307656361,1.7582314793,-0.0102206832 Al,0,1.4051732147,0.2609469472,-2.0190148504 Al,0,2.7862515281,-1.2080793025,0.0073408572 Al,0,-3.2926034865,-0.7021929171,0.0313754127 Al,0,-0.0655890245,-1.0717613946,0.0098662776 Al,0,-1.5093415342,0.1335114859,2.0281596277 Al,0,-1.5303173996,0.0829441716,-2.020082334 C,0,-1.5982918866,0.4519921071,-0.0022175722 C,0,1.5195779583,0.4258808162,-0.0049025299 $\mathrm{Na}, 0,2.6849150549,2.5824376369,-0.0387121086$ $\mathrm{Na}, 0,0.4902315967,-2.634559976,-2.4415250718$ $\mathrm{Na}, 0,-2.6609633413,2.7045741226,-0.0437783714$ $\mathrm{Na}, 0,0.4981884102,-2.5970511159,2.4795576693$ HF $=-\mathbf{2 6 6 4 . 9 5 1 7 5 0 1}$ 


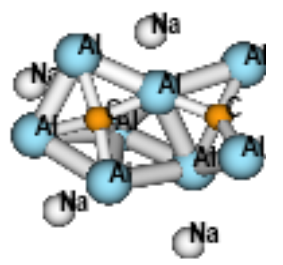

Al,0,1.0684638673,-0.0065848923,-1.1513691477 Al,0,-0.5499934206,-1.081143623,0.6989834693 Al,0,-0.0049610771,2.0118787797,0.7551427997 Al,0,2.6061929461,1.2136699851,1.0373441779 Al,0,-2.4299448906,-2.8186130088,-0.4491195301 Al,0,-1.6644235338,0.5227759336,-1.2063199377 Al,0,1.8649999164,-1.3833872675,1.7118103113 Al,0,-3.7745086236,0.2499465818,0.7368161805 C,0,-2.3936483716,-0.9407236031,0.0452313771 C,0,0.8029028171,0.27736698883,1.1668274832 $\mathrm{Na}, 0,-2.7726426389,3.1053877587,-0.0485459887$ Na,0,3.9919156352,-0.725588054,-0.8041179474 $\mathrm{Na}, 0,2.1141983535,2.7347411589,-1.6115909614$ $\mathrm{Na}, 0,0.8949759365,-3.2091265257,-0.8452569546$ HF $=-\mathbf{2 6 6 4 . 9 5 1 2 9 0 4}$

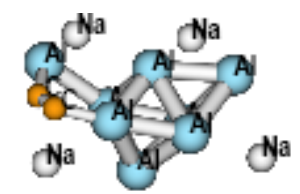

Al,0,-0.0014789108,-1.8182703493,1.3085937667 Al,0,-3.2481421658,0.5900550292,-0.9884551145 Al,0,0.8670480488,0.7536289817,1.4303994252 Al,0,-1.7082587928,0.1225520392,1.4285512245 Al,0,-0.4778806617,0.8533587329,-0.9617170796 Al,0,0.9964180399,-1.2693448081,-1.0369536526 Al,0,-1.6821246317,-1.6252735932,-0.7590000357 Al,0,3.0862928321,1.8636546951,0.2586929731 C,0,2.9354588075,-0.935282017,-1.3292404722 C,0,3.4139364426,-0.0072354154,-0.6144527727 $\mathrm{Na}, 0,-1.9714743902,2.9945103087,0.5453092304$ Na,0,-4.2094625264,-1.5845075584,0.9215155078 $\mathrm{Na}, 0,3.0866979995,-1.5295094838,1.366907767$ $\mathrm{Na}, 0,2.1932634303,1.2595444732,-2.5773043344$ HF $=-\mathbf{2 6 6 4 . 9 4 2 6 3 9}$

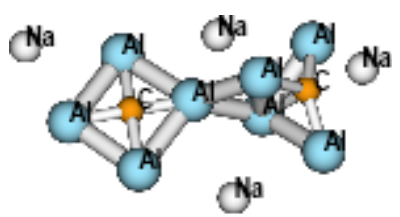

Al,0,4.4012058578,0.9499093751,0.3454424002 
Al,0,2.8448490116,-1.4005761674,0.1392560991 Al,0,2.1669155909,2.4548675142,0.1607747343 $\mathrm{Al}, 0,0.5755107381,0.1364344355,0.239974104$ C,0,2.4836273866,0.5154262564,0.3117824295 C,0,-3.0388414597,-0.2680863137,0.1826948796 Al,0,-1.5272401606,0.0055906514,-1.3428823854 Al,0,-1.7087608539,-0.1424435818,1.6638077668 Al,0,-3.5932727713,1.5485655159,-0.432010769 Al,0,-3.0876677959,-2.1209555061,-0.540083013 $\mathrm{Na}, 0,5.9059109955,-1.6150772233,0.1847757819$ $\mathrm{Na}, 0,-0.7633480501,2.9265931963,-1.0731726542$ Na,0,-0.0202092133,-2.6233030964,-1.2414211078 $\mathrm{Na}, 0,-4.9040565122,-0.5147709432,1.5832279767$ HF $=-\mathbf{2 6 6 4 . 9 3 9 9 3 2 8}$

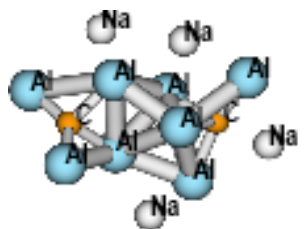

Al,0,-0.7227875238,-1.3059456781,-0.8443629557 Al,0,-1.5913521241,1.3296346919,-1.7069977889 Al,0,-3.0514932809,-0.9054328703,1.2648980176 Al,0,-0.8463378037,0.8529557083,0.9911423146 Al,0,2.8912324959,2.0972839953,0.4679609517 Al,0,1.267039868,-0.7928721717,1.0183253096 Al,0,0.9580323143,0.6602441872,-1.2287998072 Al,0,3.7408740144,-1.1785510947,-0.6586085421 C,0,-2.2066970582,-0.086190549,-0.2565869683 C,0,2.5959140583,0.2800174369,-0.127729683 $\mathrm{Na}, 0,0.0811323754,3.5798383817,-0.0383689473$ Na,0,-0.6873021714,-1.0703879993,3.3308417409 $\mathrm{Na}, 0,-4.1194717138,-0.0578016174,-1.7148366446$ $\mathrm{Na}, 0,1.387186466,-3.4523832477,-0.544940475$ HF $=-\mathbf{2 6 6 4 . 9 5 6 8 8 1 6}$

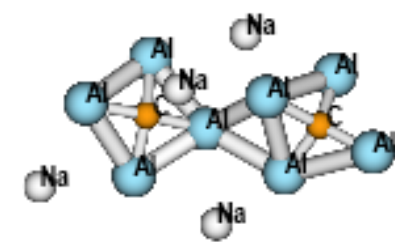

C,0,2.0870939804,-0.475331692,0.043294771 Al,0,3.9344656698,-1.0186685203,0.4619489099 Al,0,0.2410704581,0.1445250846,-0.4470238796 $\mathrm{Al}, 0,1.8241808866,-2.2362840118,-0.8772549913$ 
Al,0,2.6603087726,1.4048721142,-0.2756489656

Al,0,-2.0981718753,1.7622132331,-0.0882439968 Al,0,-3.9310303795,-1.1796316384,-0.7669667095 Al,0,-1.7871907417,-0.7450194024,0.9963290096 Al,0,-4.9654256846,1.3141498298,0.8935665786 C,0,-3.3668212683,0.3220966614,0.3838490168 $\mathrm{Na}, 0,5.6371906284,1.4833301311,0.3576711935$ Na,0,-1.2349599092,-2.6893455898,-1.4917600663 $\mathrm{Na}, 0,0.9258396428,-1.2731871153,2.1698519694$ $\mathrm{Na}, 0,0.2411724879,3.217327413,-1.1466758369$

\section{HF $=-2664.9430006$}

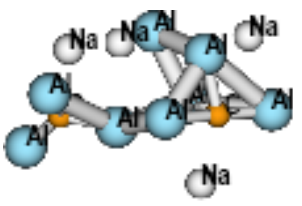

C,0,2.2305725792,-1.0819660114,0.202711124 Al,0,0.2888897872,2.1784767584,-0.2585720061 Al,0,1.0484243733,-0.15282703,-1.3337593771 Al,0,0.6585079756,-1.2781513173,1.4137250027 Al,0,-1.2638691838,-0.8898089124,-0.3415513164 Al,0,-3.0859991427,0.0129470257,1.815731997 Al,0,2.1380696377,0.8339179003,0.9931766498 Al,0,-4.5012223334,-0.9158130721,-1.1763721145 Al,0,3.878321083,-0.9522083341,-0.7629095155 C,0,-3.0663703459,-0.2804824813,-0.0737240918 $\mathrm{Na}, 0,-2.6723557597,2.098879603,-0.7194267429$ $\mathrm{Na}, 0,-0.3474320852,1.1344132428,2.9708149595$ Na,0,1.2480048068,-3.071295726,-0.9578744949 $\mathrm{Na}, 0,3.2190737691,1.9561630362,-1.7768794809$ HF=-2664.9470533

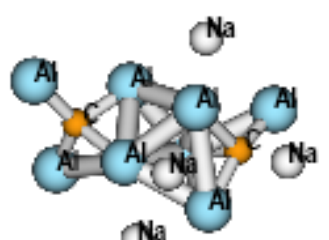

C,0,-2.16521907,-0.018838279,0.3454325847 Al,0,-0.5029504667,0.4569142935,1.4568571774 Al,0,-2.9767557458,1.6631619832,0.7408322118 Al,0,-1.0109891211,0.4338800591,-1.3516430411 Al,0,-1.4960787961,-1.9656430219,0.49463139 Al,0,1.2255104562,1.6108754454,-0.241446148 Al,0,3.8473484413,0.7623136708,-1.7676713585 Al,0,1.0494317344,-1.0176111702,-0.3493362138 Al,0,3.3555911193,-0.5037280734,1.3434218401 
C,0,2.6604326127,0.1859360862,-0.3316589623

$\mathrm{Na}, 0,0.8637548983,-2.0039411404,2.7262938619$

$\mathrm{Na}, 0,-0.7159552574,3.7062821614,0.3089244091$

$\mathrm{Na}, 0,-3.8969930501,-1.0279014301,-0.9667956143$

$\mathrm{Na}, 0,-0.6467775303,-2.4675948879,-2.4607897375$

HF $=-\mathbf{2 6 6 4 . 9 5 5 4 3 2 1}$

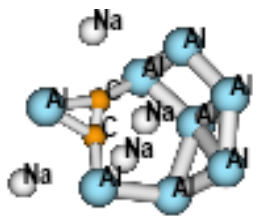

Al,0,1.9246362393,-1.9080199256,-0.0513877246 Al,0,-3.3603471606,0.091239276,-1.0869017853 Al,0,-2.1527611744,2.2859915753,-0.6206790221 Al,0,-2.6151913525,-2.4069417814,-0.9379063907 Al,0,3.0988985019,1.3200877076,-0.4052895148 Al,0,-1.9947684961,-0.3358477164,1.0681574033 Al,0,-0.5888699123,-2.6833521921,0.7381356052 Al,0,-0.5561528024,1.8239580032,1.3721577016 C,0,1.6235810628,-0.014691553,0.3147469133 C,0,1.1694617031,1.2398520975,0.3979270931 $\mathrm{Na}, 0,-0.1888922045,-0.1582050973,-1.6002073973$ $\mathrm{Na}, 0,0.6758359575,-0.6939191754,2.6955412$ $\mathrm{Na}, 0,0.8209028781,3.6134994247,-0.3878243448$ $\mathrm{Na}, 0,4.5486055004,-1.2871440222,-1.1863972381$ HF $=-2664.9116922$

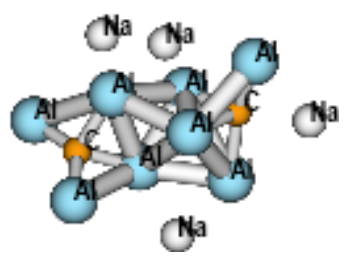

C,0,2.6003413436,0.2557522934,-0.1034117783 Al,0,-1.5803403427,1.7417870713,-1.2926362983 Al,0,0.9561109929,0.9260640259,-1.0447278344 Al,0,1.2643484984,-1.049187876,0.7609247449 Al,0,-0.7624745769,-1.0418228838,-1.1314816878 Al,0,-0.8107901352,0.5911105486,1.1875109432 Al,0,2.9328920314,1.8559357269,0.9330068506 Al,0,-3.0512071931,-1.1388808246,1.0523549945 Al,0,3.7072836298,-1.0400821477,-1.0062298698 C,0,-2.2093270334,0.0204780374,-0.2306387703 $\mathrm{Na}, 0,-4.150675818,0.4664043915,-1.5805060516$ $\mathrm{Na}, 0,0.1582451814,3.4714738835,0.8618383396$ $\mathrm{Na}, 0,-0.6656055212,-1.8594504392,2.9671790544$ $\mathrm{Na}, 0,1.3060558287,-3.2276450462,-1.4266095849$ 


\section{$H F=-2664.9568844$}

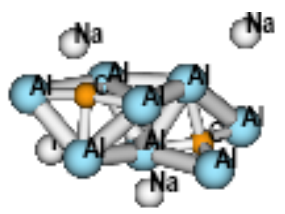

Al,0,2.0124135179,2.3426752512,-0.4667134842 Al,0,0.4549442835,0.6487484737,1.3224154447 Al,0,1.6912502872,-0.8627785951,-0.6153370184 Al,0,4.0177116227,0.0198373438,1.0339542612 Al,0,-3.5694961167,-1.2005271541,0.4085358103 Al,0,-2.1120410773,1.2016219834,0.5619516134 Al,0,-0.51542132,0.3345021563,-1.5330730634 Al,0,-0.7040981364,-1.6010961733,0.4756546948 C,0,2.2807823571,0.742828914,0.6096196715 C,0,-2.0478043659,-0.5608459331,-0.6739117044 $\mathrm{Na}, 0,-0.7455426099,3.4073601845,-1.0361316963$ $\mathrm{Na}, 0,-2.038787173,-0.4312825465,3.1155575833$ $\mathrm{Na}, 0,-2.9246052692,-1.5875499072,-2.5600918668$ $\mathrm{Na}, 0,4.074727076,-2.5313168764,-0.8875426713$ HF $=-\mathbf{2 6 6 4 . 9 5 5 1 9 0 4}$ 


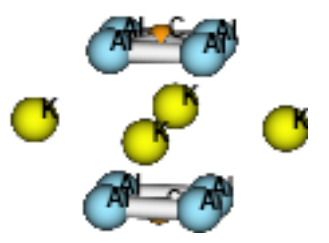

Al,0,1.9449880284,0.,-2.3914896075

Al,0,0.,-1.9449880284,-2.3914896075

Al,0,-1.9449880284,0.,-2.3914896075

Al,0,0.,1.9449880284,-2.3914896075

Al,0,0.,1.9449880284,2.3914896075

Al,0,-1.9449880284,0.,2.3914896075

Al,0,0.,-1.9449880284,2.3914896075

$\mathrm{Al}, 0,1.9449880284,0 ., 2.3914896075$

C,0,0.,0.,2.6641286665

C,0,0.,0.,-2.6641286665

$\mathrm{K}, 0,2.6424856077,2.6424856077,0$.

$\mathrm{K}, 0,-2.6424856077,-2.6424856077,0$.

$\mathrm{K}, 0,-2.6424856077,2.6424856077,0$.

$\mathrm{K}, 0,2.6424856077,-2.6424856077,0$.

HF $=-\mathbf{4 4 1 5 . 4 0 8 6 2 0 6}$
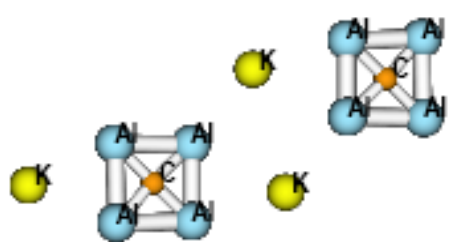

$\mathrm{K}, 0,0.1356095461,2.2856144851,0.0025955427$

$\mathrm{K}, 0,-0.1352413734,-2.2859752874,-0.001781476$

K,0,-8.8391401719,0.8269362879,0.002369492

K,0,8.838596283,-0.8282358748,-0.0043616034

$\mathrm{Al}, 0,-5.408007116,1.2941598039,0.0031594259$

Al,0,-3.6664472098,-2.239874978,-0.0005656838

Al,0,-6.2566413312,-1.458661413,0.0005762283

$\mathrm{Al}, 0,-2.819939288,0.4059528141,0.0021575641$

$\mathrm{Al}, 0,3.6673243233,2.2406938247,0.0009670537$

Al,0,5.4072368217,-1.2942008514,-0.0030991302

Al,0,6.2571868517,1.4583725008,-0.0010890606

Al,0,2.8194712041,-0.4041597127,-0.0007647609

C,0,-4.5576734219,-0.4723881754,0.0019723248

C,0,4.5578306356,0.472701767,-0.001148729

$\mathbf{H F}=-\mathbf{4 4 1 5 . 3 8 4 5 5 0 1}$ 


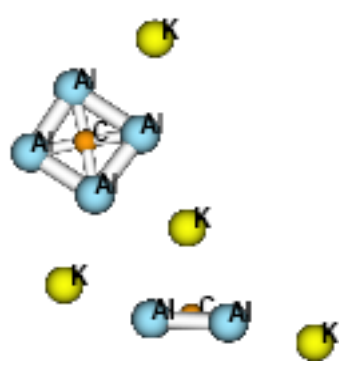

Al,0,-4.6560937003,-3.1860603394,0.

Al,0,-2.1495009683,-4.3540706715,0.

Al,0,-3.4011996138,-0.5955984741,0.

$\mathrm{Al}, 0,-0.9935564888,-1.8637364989,0$.

C,0,-2.8402483712,-2.4686078951,0.

C,0,2.8462599951,2.204459035,0.

$\mathrm{Al}, 0,3.1780092848,0.8156949644,1.4001182494$

Al,0,2.8991902138,3.5405535396,-1.4453436177 Al,0,3.1780092848,0.8156949644,-1.4001182494 Al,0,2.8991902138,3.5405535396,1.4453436177 K,0,-6.9088330457,-0.6102001731,0.

$\mathrm{K}, 0,2.424580216,-2.4497915085,0$.

$\mathrm{K}, 0,-0.2865161422,1.585054504,0$.

$\mathrm{K}, 0,3.1303860766,6.6931956355,0$.

\section{HF $=-\mathbf{4 4 1 5} .3771931$}

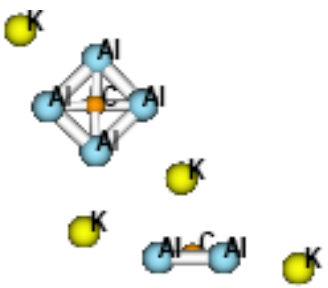

Al,0,-5.1249121697,-2.0152152943,0.

Al,0,-3.0855019392,-4.0538132149,0.

Al,0,-3.1953725798,-0.1159300536,0.

Al,0,-1.2158182473,-2.0580488467,0.

C,0,-3.1636574278,-2.0959962508,0.

C,0,3.1432708852,2.0177601953,0.

$\mathrm{Al}, 0,3.3123835865,0.6139282288,1.4062436897$ Al,0,3.3108063477,3.3503914638,-1.4417861718 Al,0,3.3123835865,0.6139282288,-1.4062436897 $\mathrm{Al}, 0,3.3108063477,3.3503914638,1.4417861718$

K,0,-6.3356716317,-5.2428851399,0.

$\mathrm{K}, 0,2.2429990156,-2.536087128,0$.

$\mathrm{K}, 0,3.7395006516,6.4826576965,0$.

$\mathrm{K}, 0,-0.0678677653,1.5361146057,0$.

\section{HF $=-\mathbf{4 4 1 5 . 3 8 0 4 8 1 4}$}




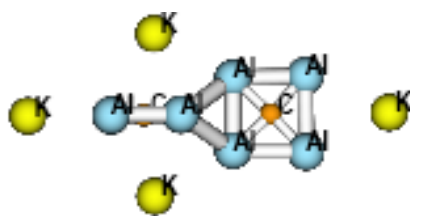

Al,0,4.0887082519,1.3810317477,0.

$\mathrm{Al}, 0,2.3268340429,3.6347982642,0$.

Al,0,1.894423556,-0.2494549345,0.

Al,0,0.2149186883,1.8989104863,0.

C,0,2.181408883,1.7055582461,0.

C,0,-1.5903522438,-1.2429763595,0.

Al,0,-0.4748807475,-0.3707741479,1.440930969

Al,0,-2.5553909852,-1.9976366835,-1.52930186

Al,0,-0.4748807475,-0.3707741479,-1.440930969

Al,0,-2.5553909852,-1.9976366835,1.52930186

$\mathrm{K}, 0,5.6698315704,4.4323031276,0$.

$\mathrm{K}, 0,0.6178149556,-3.4004389642,0$.

$\mathrm{K}, 0,-5.0091987083,-3.917984018,0$.

$\mathrm{K}, 0,-3.1512253858,1.4205660635,0$.

\section{$\mathbf{H F}=-\mathbf{4 4 1 5} .3715863$}

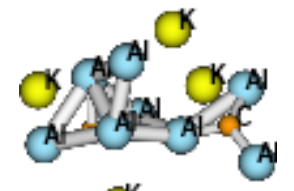

C.

C,0,-2.2099426591,0.8181658226,0.8018874496 Al,0,-0.1812224019,-2.2045289835,-0.3549328272 Al,0,-1.1306707236,0.2389231668,-0.9419311978 Al,0,-0.5279655256,0.9898766244,1.8681553597 Al,0,1.1885827533,1.1686481639,-0.1268649476 Al,0,3.7384728245,0.3148062647,1.2654951454 Al,0,-1.8839486738,-1.1743957781,1.2795598192 Al,0,3.992082787,2.1127745182,-1.6179986802 Al,0,-3.9438593727,0.698448455,0.0235563514 C,0,3.0813655204,0.9736665606,-0.3931169565 K,0,2.7103264443,-1.4750165516,-2.0252320104 K,0,1.2967663568,-1.8191082201,2.9798391532 K,0,-1.684349082,3.5299499537,-0.1983791455 K,0,-3.4541999739,-2.268992335,-1.8398138003 HF $=-\mathbf{4 4 1 5 . 3 8 3 6 9 9 7}$

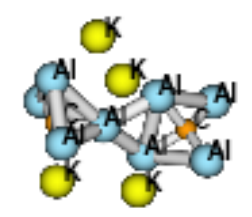

C,0,-2.2768798517,-0.6287320541,-0.4518369405 
Al,0,-2.7854999675,0.6633199559,-1.8369065317

Al,0,-0.3681589914,-0.2599559941,-0.1939995585

Al,0,-2.0921012419,-2.004445363,1.0071592159

Al,0,-3.082670953,0.6441980176,0.9560310815

Al,0,1.3870030329,1.453455677,0.4717426697

Al,0,3.9805219879,-0.6646391222,0.4149949186

Al,0,1.7908734073,-0.4465330255,-1.5441097137

Al,0,3.9063461772,2.4369245722,-0.9581826985

C,0,3.0282251298,0.7643195458,-0.498405362

K,0,-1.4458314936,3.5546239919,-0.4128916057

$\mathrm{K}, 0,1.3244651474,-3.1428743072,1.2077560688$

$\mathrm{K}, 0,-1.2081068983,-2.1874638629,-2.8326751448$

$\mathrm{K}, 0,-0.7800081522,0.4860433212,3.4895986729$

HF=-4415.3720006

$\mathrm{O}$

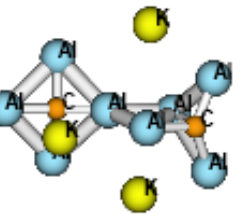

Al,0,-3.5839578091,-1.4095014479,-0.0100032893 Al,0,-2.4138613795,1.0945003723,-0.6129186279 Al,0,-1.2385361839,-2.6194280656,-0.7285434499 $\mathrm{Al}, 0,0.1257607219,-0.0712595484,-0.2830426155$ C,0,-1.73392013,-0.7411686727,-0.3325559692 C,0,3.5388922423,0.666164588,0.9268939954 Al,0,2.4506916422,0.6757063127,-1.0128099949 Al,0,1.8889842213,0.0294047261,1.7559764586 Al,0,4.5965073881,-0.808288619,0.2631770491 Al,0,3.5496442647,2.6093179911,0.7291157286 $\mathrm{K}, 0,-5.9682589327,1.1085635097,-0.2722156629$ $\mathrm{K}, 0,2.3095416643,-2.8303612064,-1.6886054707$ $\mathrm{K}, 0,0.4645538691,3.5015608068,-1.0662539646$ K,0,-1.053618702,-1.414281419,2.7703174918 HF $=-4415.3673015$

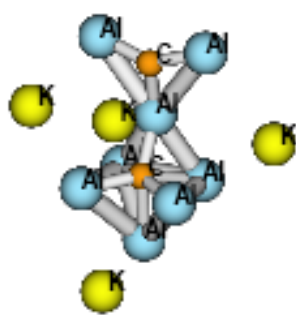

Al,0,-0.5716279411,-1.3634322711,-2.0914324532 Al,0,-2.6959229553,-0.0174939941,-1.4055361351 Al,0,-0.5862897485,1.3534286798,-2.0870273569 Al,0,-1.3796014757,-1.5939261964,0.6075497537 Al,0,1.2452879631,0.0076795774,-0.7211624615 
Al,0,3.4224061795,-1.7695584197,0.3914771394

Al,0,-1.4012582085,1.5688991895,0.6075006691

Al,0,3.3850591828,1.8292229576,0.3867690348

C,0,2.8381728195,0.023699437,0.4073782878

C,0,-0.7125533931,-0.0075825363,-0.3587849758

K,0,-4.4459265811,-0.0379427179,1.5172458778

$\mathrm{K}, 0,0.9294947114,0.0011809592,2.7471265811$

$\mathrm{K}, 0,0.9483528505,-4.1220203073,-0.68222879$

K,0,0.869096373,4.1442052908,-0.6626964067

HF =-4415.4015904 


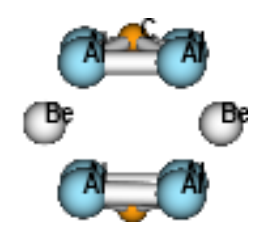

Al,0,1.9030799229,-0.0213675684,-1.7902675412 Al,0,0.0213675684,-1.9030799229,-1.7902675412 Al,0,-1.9030799229,0.0213675684,-1.7902675412 Al,0,-0.0213675684,1.9030799229,-1.7902675412 Al,0,-0.0213675684,1.9030799229,1.7902675412 Al,0,-1.9030799229,0.0213675684,1.7902675412 Al,0,0.0213675684,-1.9030799229,1.7902675412 Al,0,1.9030799229,-0.0213675684,1.7902675412 C,0,0.,0.,2.3012892637 C,0,0.,0.,-2.3012892637 Be,0,1.6922127006,1.6922127006,0. Be,0,-1.6922127006,-1.6922127006,0.

\section{HF $=-\mathbf{2 0 4 5 . 0 8 3 3 3 8 2}$}

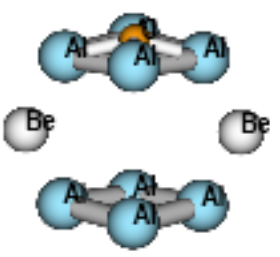

$\mathrm{Al}, 0,1.8231284846,0 .,-2.0174542116$ $\mathrm{Al}, 0,0 .,-2.015054708,-2.1460582625$ Al,0,-1.8231284846,0.,-2.0174542116 $\mathrm{Al}, 0,0 ., 2.015054708,-2.1460582625$ Al,0,0.,2.015054708,2.1460582625 $\mathrm{Al}, 0,-1.8231284846,0 ., 2.0174542116$ Al,0,0.,-2.015054708,2.1460582625 $\mathrm{Al}, 0,1.8231284846,0 ., 2.0174542116$ C,0,0.,0.,2.4924190983 C,0,0.,0.,-2.4924190983 Be,0,2.8255221258,0.,0. Be,0,-2.8255221258,0.,0.

\section{HF $=-\mathbf{2 0 4 5 . 0 2 3 3 1 7 2}$}

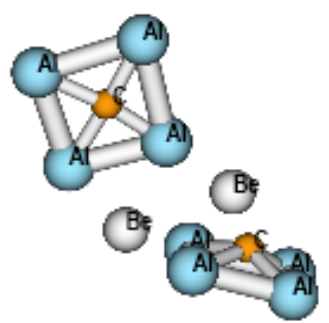

Al,0,-2.3125113338,-1.6460576673,0.05448667019 Al,0,-1.1001775501,0.7297203352,-0.0291614541 $\mathrm{Al}, 0,-4.8131632849,-0.5418955223,0.0152785933$ 
Al,0,-3.6313072121,2.1211516028,-0.0759038849 Al,0,4.0648262919,0.6145231092,1.3624384045 Al,0,1.7714981856,-0.9015113917,1.4235373194 Al,0,1.7755700443,-0.9978393835,-1.3546584546 Al,0,4.0686149658,0.5191930116,-1.3918748537 C,0,-2.9294256119,0.2340324207,-0.0114253069 C,0,2.561765015,0.3716417618,-0.0100836941 Be,0,0.0077257434,-1.6902707042,0.0572352786 Be,0,1.1178773052,1.1155861251,-0.0384344856 HF $=-\mathbf{2 0 4 5 . 1 4 5 3 9 8 9}$

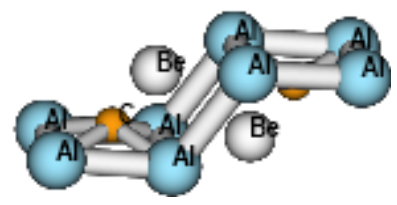

$\mathrm{Al}, 0,-1.64267342,0.291160628,3.5849488563 \wedge \mathrm{M}$ $\mathrm{Al}, 0,-0.4195033991,1.4172215614,1.4312639375 \wedge \mathrm{M}$ $\mathrm{Al}, 0,-0.0753345504,-1.961248806,3.4324304172^{\wedge} \mathrm{M}$ $\mathrm{Al}, 0,1.2686153908,-1.0085225319,1.2671565013 \wedge \mathrm{M}$ $\mathrm{Al}, 0,1.6430071229,-0.2910925552,-3.5846286926 \wedge \mathrm{M}$ $\mathrm{Al}, 0,0.4196634512,-1.4170886616,-1.4310569042^{\wedge} \mathrm{M}$ $\mathrm{Al}, 0,-1.268804595,1.008443959,-1.2673538629 \wedge \mathrm{M}$ $\mathrm{Al}, 0,0.0751283165,1.9609884279,-3.4327699125 \wedge \mathrm{M}$ C,0,-0.5677097753,-0.533089943,2.0428562902^M $\mathrm{C}, 0,0.5676250624,0.5332053512,-2.0428461303^{\wedge} \mathrm{M}$ Be,0,-1.0582285976,-0.7679274176,0.467413716^M $\mathrm{Be}, 0,1.0580361366,0.7682027351,-0.4673975608 \wedge \mathrm{M}$

\section{HF $=-\mathbf{2 0 4 5 . 1 7 0 2 3 5 6}$}

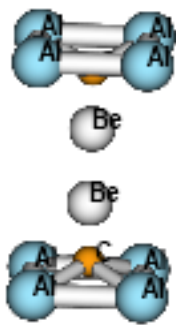

$\begin{array}{rcccc}6 & 0 & 0.000000 & 0.000000 & 2.666830 \\ 4 & 0 & 0.000000 & 0.000000 & 1.031819 \\ 13 & 0 & 0.000000 & 2.034993 & 3.235840 \\ 13 & 0 & -2.034993 & 0.000000 & 3.235840 \\ 13 & 0 & 0.000000 & -2.034993 & 3.235840 \\ 13 & 0 & 2.034993 & 0.000000 & 3.235840 \\ 4 & 0 & 0.000000 & 0.000000 & -1.031819 \\ 6 & 0 & 0.000000 & 0.000000 & -2.666830 \\ 13 & 0 & 0.000000 & 2.034993 & -3.235840 \\ 13 & 0 & 2.034993 & 0.000000 & -3.235840 \\ 13 & 0 & 0.000000 & -2.034993 & -3.235840\end{array}$




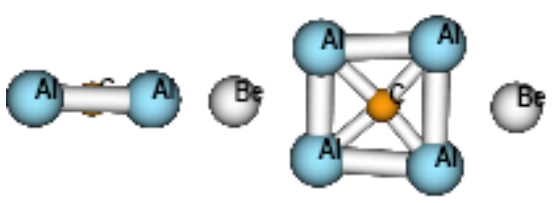

Be,0,0.444522138,-4.2260117868,4.8546702489 Al,0,1.3403413645,-2.1489239559,4.0444807464 Al,0,-0.7139096089,-3.8019440497,2.792308545 Al,0,1.052231043,-0.4197444096,1.907845693 Al,0,-0.8033728248,-1.9112895794,0.7751321283 C,0,0.226502446,-2.1488398082,2.4691673401 Be,0,-0.0136223402,0.1679142201,-0.1871794009 C,0,-0.2509727458,2.3750191088,-2.7299533785 Al,0,0.7935106402,0.7780178974,-2.3265284203 Al,0,-1.3922685707,4.0061008979,-3.0202089387 Al,0,-1.0949739601,2.1133585319,-0.9911877812 $\mathrm{Al}, 0,0.6971513482,2.528679626,-4.4976302157$

\section{HF $=-\mathbf{2 0 4 5 . 0 6 0 0 8 3 1}$}

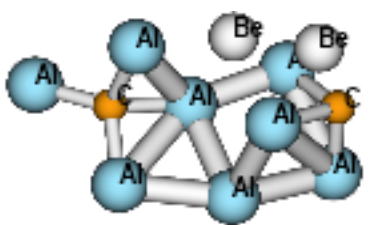

Al,0,-1.9379615486,-1.6697561153,0.6367287518 Al,0,-0.4102735734,0.4462149837,0.71298513 Al,0,-1.9866529003,1.214858358,-1.3979498274 Al,0,-4.1433292044,0.5699338893,1.0140454619 Al,0,3.1856001236,1.0638223348,-0.5974909173 Al,0,1.9144827944,-0.0235304538,1.6702765571 Al,0,1.4337986265,-1.317923659,-1.1666850525 Al,0,0.7348470966,1.1391277434,-1.5669673448 C,0,-2.3412452834,0.2716120822,0.3050514908 C,0,3.0618181548,-0.7318435352,0.0129702248 Be,0,0.3943017616,-1.8886360364,1.0228717666 Be,0,2.4556768347,-2.0449447981,0.7590316941 HF $=-\mathbf{2 0 4 5 . 1 4 1 3 9 1 5}$

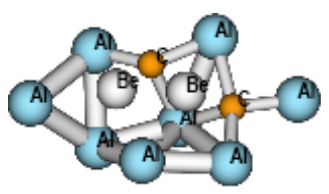

Al,0,-0.9928252847,1.5246870699,-1.1871425126 Al,0,-3.9368159811,-0.3110663589,0.5816910126 Al,0,-1.7703778912,1.0969921487,1.4499975923 
Al,0,-1.7316492601,-1.7608239478,1.3162850317 Al,0,1.5601005649,1.7511014498,-1.7344774556 Al,0,3.9950687164,-0.0115440884,-0.6587330775 Al,0,1.7356773758,-1.9769405614,0.1554671867 Al,0,0.6950676681,0.555690449,0.9329204424 Be,0,0.4531975481,-0.4399991167,-1.1994580005 C,0,1.9914213849,0.1165859019,-0.6188024186 C,0,-0.2754966869,-1.2826475582,0.09275545 Be,0,-1.5783837964,-0.6322209214,-0.7934982618 HF=-2045.1667699

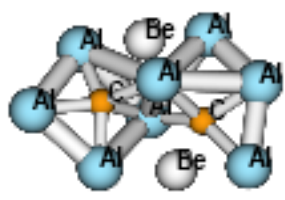

Al,0,2.358452118,-1.5964238954,-0.0585585233 Al,0,-0.1571217678,-0.3081233928,1.1534932571 Al,0,1.461056294,2.1712255263,-0.0893283586 $\mathrm{Al}, 0,3.5324090702,0.6975542186,0.6693344709$ Al,0,-3.1136768585,-0.594779346,0.527842659 Al,0,0.2862069384,-0.0795637522,-1.4611706143 Al,0,-1.38973858,-2.2862399682,-0.1061421875 Al,0,-2.9638066592,1.8771797371,-0.0183028692 C,0,-1.4557141728,0.3968785465,-0.3178101267 C, $0,1.5458463401,0.2064644457,0.2453614668$ Be,0,-0.7679547175,1.8915714867,-0.6754422072 Be,0,0.5879696626,-2.4092806397,-1.2216802637 HF=-2045.1290472

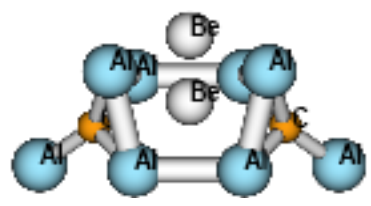

Al,0,1.7215505531,0.,-2.0067172655

Al,0,-0.194263606,-1.6796072302,-1.3843238156 Al,0,-1.7377616074,0.,-3.7733165515 Al,0,-0.194263606,1.6796072302,-1.3843238156 Al,0,-0.194263606,1.6796072302,1.3843238156 Al,0,-1.7377616074,0.,3.7733165515 Al,0,-0.194263606,-1.6796072302,1.3843238156 $\mathrm{Al}, 0,1.7215505531,0 ., 2.0067172655$ C,0,-0.3359171569,0.,2.4118899599 C,0,-0.3359171569,0.,-2.4118899599 Be,0,1.8192751005,1.1778160383,0. Be,0,1.8192751005,-1.1778160383,0. HF $=-2045.1114643$ 


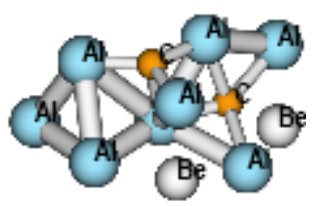

Al,0,3.492264219,-0.5144769507,-1.4969683785 Al,0,0.1895614797,0.2700876859,-0.7108486198 Al,0,-1.3930022758,-1.5401533176,0.323760534 Al,0,2.1535700707,-1.5142260207,0.616408184 Al,0,-1.8190226709,2.1653045999,-0.1464062983 Al,0,0.0514443671,0.5086696082,2.2584940417 Al,0,2.3950239355,1.2772919453,0.194970742 Al,0,-3.5385623782,-1.2396404928,-0.9387492581 C,0,-1.7984414988,0.1149862881,-0.8340098383 C,0,0.3537412971,-0.9758066677,1.0287283694 Be,0,-3.1323134016,0.9126549545,-1.3877542452 $\mathrm{Be}, 0,0.3227142758,2.2867901779,0.7685283711$ HF $=-2045.1274652$

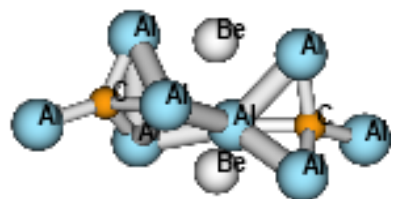

C,0,0.0569368072,-1.870590833,1.9850519856 Al,0,-0.1752775142,-3.7112118851,2.6475556787 $\mathrm{Al}, 0,-1.2004617677,-0.588060733,2.774428327$ Al,0,-0.2809779832,-0.8453727531,0.2611664584 Al,0,1.8989743661,-1.2557164147,1.7192637931 Be,0,-1.4114333937,1.1088132674,1.172377449 Al,0,0.1080458358,1.9929648423,-0.1996679718 Al,0,-1.1397882301,-0.0179010016,-2.0464218309 Al,0,1.6683170436,0.9634795515,-2.1079313535 Al,0,-0.9884361867,3.0942330689,-3.311651988 C,0,-0.1708829409,1.6870419751,-2.221301798 Be,0,1.9385670124,0.361162325,0.0375886524 HF=-2045.1204868

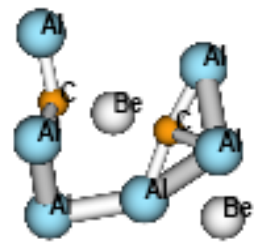

Be,0,-0.6650317248,-0.2587455845,0. Al,0,2.2830690364,-1.9025362079,0. Al,0,0.1900142261,-2.3137372072,1.6350498377 Al,0,-2.7991092113,-2.3556634918,0. 
Al,0,0.1900142261,-2.3137372072,-1.6350498377

Al,0,0.2737177658,2.7094570677,-1.5007826319

Al,0,-2.0559667062,2.2042323624,0.

Al,0,0.2737177658,2.7094570677,1.5007826319

Al,0,1.7070511452,0.7212881369,0.

C,0,-0.2397920332,1.3210905263,0.

C,0,-0.8792336952,-1.9165711432,0.

Be,0,2.1404185117,2.9109948177,0.

\section{HF $=-2045.1181374$}

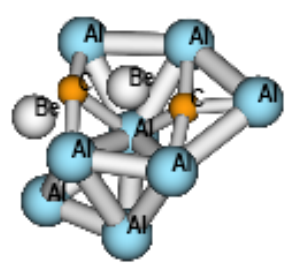

Al,0,0.4310100058,-1.9838685514,0.7126661023 Al,0,-1.5202781762,-0.2726618228,1.5059028329 Al,0,2.4789734009,1.2907721735,-0.6031875792 Al,0,3.2873103954,-1.4579167757,-0.1143453622 Al,0,-3.0586727426,0.1340659134,-0.7984864597 Al,0,-0.1673456495,0.2451591779,-0.9617135926 Al,0,-1.7329324638,-2.0513449145,-0.7879152786 Al, 0,0.2053787068,2.9037660145,0.0016001268 C,0,-0.58429217,1.4442054069,1.1328512965 C,0,1.5559701764,-0.4255296382,0.0764795424 Be,0,-1.9633921119,1.8361021747,0.3343879348 Be,0,0.7546838023,0.5099777239,1.2494232402

\section{HF $=-\mathbf{2 0 4 5 . 1 8 4 1 1 0 6}$}

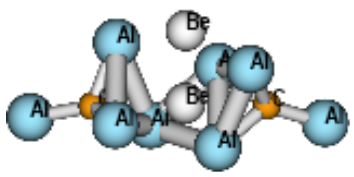

Al,0,-1.1628540476,-0.3614071677,-1.4031854091 Al,0,-2.1358166001,1.683850755,0.0179378264 Al,0,-4.2947078904,-1.2039523593,-0.0169650657 Al,0,-1.1610844481,-0.3917241923,1.3929188394 Al,0,1.8361833322,0.8679119284,1.4415246444 Al,0,0.8478834585,-1.3557159624,-0.0169248894 Al,0,1.842715021,0.9021659422,-1.4219371436 Al,0,4.387025147,-0.9778452024,-0.0035723198 C,0,-2.5952265741,-0.2651404145,-0.0028041607 C,0,2.5461425463,-0.3234644708,-0.0033100482 Be,0,-0.2206562532,1.8132334981,-1.2322844227 Be,0,-0.2235856159,1.7890016703,1.2746171683 HF $=-2045.137637$ 


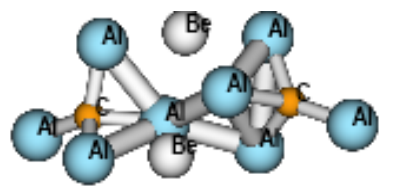

Al,0,-1.6066703269,-0.8289699088,0.8695608352 Al,0,-1.2841407804,1.9452998032,-0.2306626116 Al,0,-2.2516780744,-0.4563066786,-1.6960119677 Al,0,-4.433452941,1.1882691107,0.6689364369 Al,0,4.5248784913,0.2746019188,-0.5134314558 Al,0,2.3919602545,-0.4044534616,1.8983188489 Al,0,0.8176062603,0.4227324638,-0.1183403731 Al,0,1.942606409,-1.7601470486,-1.1249936067 C,0,-2.7254450384,0.6109096352,-0.0972547012 C,0,2.7083735646,-0.3209759581,-0.0365328721 Be,0,0.0791682762,-0.1069885498,2.1393011651 Be,0,-0.382166266,-1.566247112,-1.1370921503 HF $=-2045.1204863$

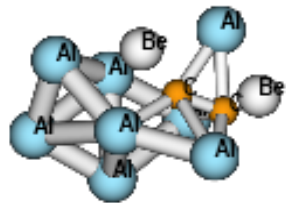

Al,0,-1.5389406191,-0.9399898578,1.4173004266 Al,0,0.5943707516,-2.2598924213,-0.052801234 Al,0,2.5595641384,1.2677482812,0.1691056683 Al,0,0.8446502053,-0.0418725604,-1.4851024002 Al,0,2.8602957654,-1.2098509986,0.3326152331 Al,0,-1.7552191815,-0.2252261448,-2.468797955 Al,0,-1.9913148823,2.2482404948,0.6185782114 C,0,-0.8487480978,0.6416636738,-0.3663149656 $\mathrm{Al}, 0,0.7601248588,0.1294566836,1.6483840566$ C,0,-2.1683963522,0.2146927823,-0.4338186958 Be,0,0.2619316444,1.8759990999,-0.1027924954 Be,0,-3.3201908385,0.1914724168,0.7203264657 HF $=-2045.1351995$

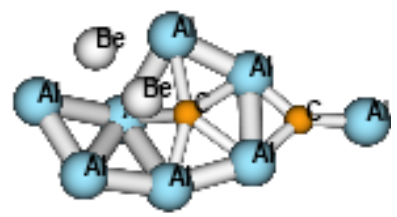

Al,0,-1.8143235374,2.5157678218,-1.3369538874 Al,0,-3.9110093916,1.2621909031,-0.6727922213 Al,0,-1.5835505754,0.8432140917,0.8065125068 Al,0,-1.2994381084,-1.9348216612,0.3989525128 Al,0,0.4751086956,1.8724793257,-0.5881464821 $\mathrm{Al}, 0,4.6634786673,-2.0423782692,1.048366057$ 
Al,0,2.2780233693,0.2838272947,-0.128741409

C,0,0.0472782755,-0.1948228337,-0.1390727835

Al,0,1.137873092,-1.8196279169,0.6989966728

C,0,2.9453043205,-1.3121224576,0.6094894551

Be,0,-1.2351145658,-0.1697005142,-1.2330052552

Be,0,-3.0787865154,-0.756999215,-0.2077494385

HF $=-2045.1249369$

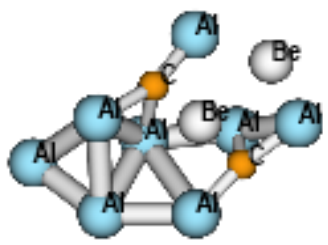

Al,0,-2.3064643989,1.07474426637,-1.4071990434 Al,0,-3.902365058,0.2299494053,0.4499653804 Al,0,-0.7690697374,0.5612279113,0.8972171818 Al,0,-1.8309534799,-1.467920185,-0.3539330534 Al,0,0.2611357261,1.3825566582,-1.8810883839 Al,0,3.5477595356,-0.0370644589,-0.6276722526 Al,0,1.2498481325,-2.007141984,1.7664470958 Al,0,1.7580092453,1.0749292098,1.0554285987 C,0,1.7761801074,0.669153604,-1.0330113766 C,0,-0.15796712,-1.4585983712,0.5905935111 Be,0,3.1466613092,-0.756580682,1.4859019307 Be,0,0.9003443222,-0.6959096331,-0.4945630832 HF $=-2045.1558815$

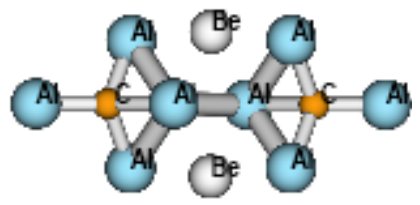

Al,0,2.3136320128,1.5986317294,-0.6283709141 Al,0,0.9494037263,-0.0082294882,0.9573525414 Al,0,2.1270718815,-1.8865188506,-0.4724660935 Al,0,4.7250733787,-0.2270022681,0.4966882745 Al,0,-4.7238272732,0.2299535031,-0.5054456447 Al,0,-2.1262827309,1.8847453252,0.4765678444 Al,0,-0.9509360457,0.0078217675,-0.9572447275 Al,0,-2.3133044693,-1.598594429,0.631749154 C,0,2.800520571,-0.1448318628,0.1328840627 C,0,-2.8018218841,0.1438046062,-0.1299462599 Be,0,0.1050731217,1.9670434176,-0.0883462454 Be,0,-0.1058202127,-1.9681262227,0.087740629 HF $=-\mathbf{2 0 4 5 . 1 3 3 1 6 3 7}$ 


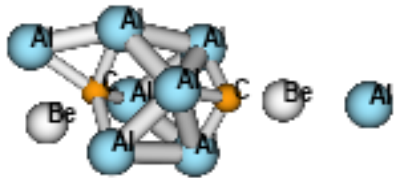

C,0,-1.7274360282,0.0281029995,0.0937894618 Al,0,1.3427816955,-1.9840189931,0.6349405921 Al,0,-0.4112805499,-0.9221408476,-1.0673156354 Al,0,-0.8352983033,1.7645122339,-0.1770488182 Al,0,1.4199723676,0.9255764199,-1.5406755899 Al,0,1.1495194988,1.1069743052,1.5792602108 Al,0,-0.8932566114,-0.7004542998,1.7180179592 Al,0,3.9081518572,0.4390375155,-1.0005050923 $\mathrm{Al}, 0,-5.8175605257,-0.3495102646,-0.4819133761$ C,0,2.0389657842,0.0246438481,0.2006285703 Be,0,3.3156053133,-0.8634917382,0.7869093528 Be,0,-3.337745591,-0.1255507588,-0.1390072141 HF $=-2045.1170713$

Al,0,0.9287298952,2.1421293002,0.3924187263 $\mathrm{Al}, 0,-2.9773027306,1.1316187454,0.4681605521$ Al,0,0.8694471687,-0.3453322755,1.3712266587 Al,0,-3.9852455029,-1.2224510662,-0.2591958416 Al,0,3.3300967398,0.8997625052,-0.1368785765 Al,0,-0.8377628283,0.6939106024,-1.244975661 Al,0,-1.4605669543,-1.4814527481,0.2831933166 Al,0,2.9456770044,-2.1715485899,0.0854589929 C,0,1.0889173932,0.4364536739,-0.8776901462 C,0,2.0161261371,-0.5970179823,-0.6776753049 Be,0,-1.286000739,2.6437146468,0.1877621884 Be,0,0.4859488697,-1.254436723,-0.9727905562 HF $=-2045.1380399$

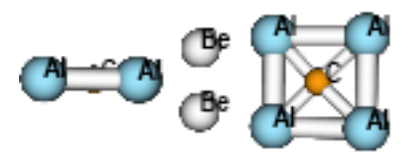

Be,0,0.6886733684,0.5997426412,0.3742028356 Al,0,1.010488962,-2.7121109803,4.1411790975 Al,0,-1.0104889517,-4.1411790932,2.7121109906 Al,0,0.9739140774,-0.7617153322,2.1390579942 Al,0,-0.973914073,-2.139057994,0.7617153382 C,0,0.0000000036,-2.3968294393,2.3968294444 Be,0,-0.6886733688,-0.3742028379,-0.5997426394 
C,0,-0.0000000036,2.3685872614,-2.3685872665 Al,0,0.9744958147,0.7303545304,-2.1084795572 Al,0,-1.0172940026,4.1487586044,-2.7101087067 Al,0,-0.974495819,2.1084795542,-0.7303545334 Al,0,1.0172939923,2.7101086994,-4.1487586117 HF $=-2045.0942783$

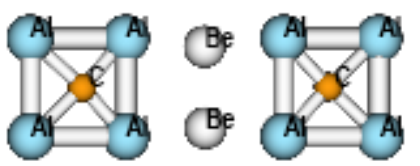

Al,0,0.9965425746,-0.9959377843,-2.2852258017 Al,0,1.0026332988,-1.0034339879,-5.0574247457 Al,0,-1.0034339879,1.0026332988,-5.0574247457 $\mathrm{Al}, 0,-0.9959377843,0.9965425746,-2.2852258017$ Al,0,-0.9959377843,0.9965425746,2.2852258017 Al,0,-1.0034339879,1.0026332988,5.0574247457 Al,0,1.0026332988,-1.0034339879,5.0574247457 Al,0,0.9965425746,-0.9959377843,2.2852258017 C,0,-0.0000162535,-0.0000162535,3.5763162895 C,0,-0.0000162535,-0.0000162535,-3.5763162895 Be, $0,0.8330425728,-0.8317204699,0$. Be,0,-0.8317204699,0.8330425728,0.

\section{HF =-2045.1105972}

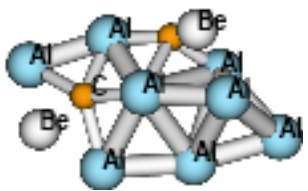

Al,0,-1.6594217209,0.9802255199,-1.0271117717 Al,0,-3.7802117493,0.0872909357,0.2466158807 Al,0,-1.6948883618,1.0993894372,1.8182276581 Al,0,-1.5925321485,-1.3821342058,0.6730896195 Al,0,0.7831687532,1.4415510459,-2.0003403439 Al,0,3.6861354777,-1.081285976,-1.2592742684 Al,0,1.8230872718,-1.5633219201,0.3326182629 $\mathrm{Al}, 0,0.5917303196,0.7603208277,0.6378710584$ C,0,2.0243571757,0.2365006528,-0.6309905789 C,0,0.2052276882,-1.2996310856,1.3579184159 Be,0,-0.2477320528,-0.423951483,2.6575159482 Be,0,2.8928842711,0.9070312224,-1.8684200147 HF $=-2045.1305354$

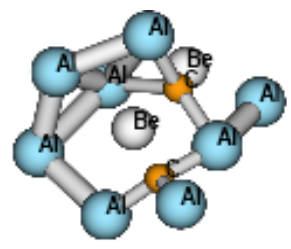


Al,0,-1.4452317019,2.2219473645,-0.9299656063 Al,0,-2.6998703868,0.1244463803,-1.3317174736 Al,0,-0.6445457197,-1.0707762445,3.4672854782 Al,0,-2.0800252071,-1.6060442652,0.6400490461 Al,0,0.0191216786,0.5616583796,-2.3539500981 Al,0,3.3422735621,-1.2245740129,-0.5027367482 Al,0,1.4156888635,-1.0599116638,1.1367717336 Al,0,1.1242784362,2.2957376026,-0.0850567803 C,0,1.3904634206,0.3193167098,-0.4345142931 C,0,-0.4816124042,-0.9802834864,1.5154460906 Be,0,2.1248033885,0.0644529441,-1.9336457271 Be,0,-0.341070869,0.1389257137,0.1800394885 HF $=-\mathbf{2 0 4 5 . 1 4 4 0 3 8 3}$

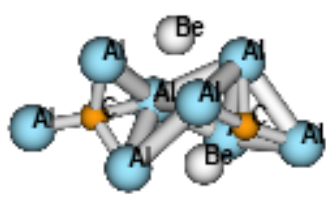

Al,0,-2.2286511706,1.6905455021,-0.1853250684 Al,0,0.5758040523,1.3927813631,1.2548145173 Al,0,-1.3385278244,-0.9202923078,1.5591209892 Al,0,-4.0812363216,-1.1046404099,-0.393483663 Al,0,1.7280594341,-1.6242970848,-1.0366261766 Al,0,-0.5366171399,-0.2994262067,-0.9245466283 Al,0,1.9479223849,1.1707798258,-1.1009015394 Al,0,3.8109995039,-0.6481297294,0.4100218606 C,0,2.059959522,0.2219231746,0.7479829043 C,0,-2.3382346406,-0.2781265537,-0.0090712029 Be,0,0.9257896925,-0.9615472178,1.1656697557 Be,0,-0.1110740004,2.1595591911,-0.9190287547 HF $=-\mathbf{2 0 4 5 . 1 4 8 2 6 4 4}$

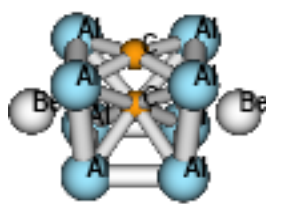

Be,0,-1.9420831992,1.9420526008,-0.2390265537 Al,0,1.9563276961,0.1377491969,-1.4165052254 Al,0,-0.1377183737,-1.9563298662,-1.4165052254 Al,0,-1.9563276961,-0.1377491969,-1.4165052254 Al,0,0.1377183737,1.9563298662,-1.4165052254 Al,0,-0.1429701982,2.0567254838,1.2806217591 Al,0,-2.0567277361,0.1429377933,1.2806217591 Al,0,0.1429701982,-2.0567254838,1.2806217591 Al,0,2.0567277361,-0.1429377933,1.2806217591 C,0,0.,0.,1.5269950356 
C,0,0.,0.,-0.0306362565

Be,0,1.9420831992,-1.9420526008,-0.2390265537

\section{HF $=-2045.0574594$}

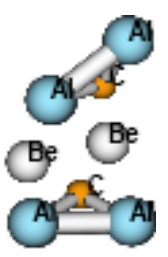

Be,0,-0.4560332994,0.4560261143,0.0226032476 Al,0,2.3240340363,-0.0854270557,-2.0381810297 Al,0,0.0854636723,-2.3240326901,-2.0381810297 Al,0,-1.6753111047,-0.2635646359,-2.3153060873 Al,0,0.2635382403,1.6753152571,-2.3153060873 Al,0,-0.1183313259,2.0992572949,2.8801090852 Al,0,-2.099259159,0.1182982508,2.8801090852 Al,0,-0.4906944911,-1.6092152744,1.4319434358 Al,0,1.609207543,0.4907198452,1.4319434358 C,0,-0.4716578881,0.4716504569,1.6967906044 C,0,0.2145843879,-0.214581007,-1.364504432 Be,0,1.1710394634,-1.1710210131,-0.251707632 HF $=-\mathbf{2 0 4 5 . 1 5 8 7 6 0 3}$

(B)

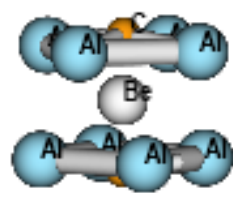

Be,0,0.,0.,0.0375732513

Al,0,-1.4520920056,1.4520920056,1.4493488149 Al,0,-1.4520920056,-1.4520920056,1.4493488149 Al,0,1.4520920056,-1.4520920056,1.4493488149 Al,0,1.4520920056,1.4520920056,1.4493488149 Al,0,0.,2.1351672079,-1.2311450344 Al,0,2.1351672079,0.,-1.2311450344 Al,0,0.,-2.1351672079,-1.2311450344 Al,0,-2.1351672079,0.,-1.2311450344 C,0,0.,0.,-1.5766012344 C,0,0.,0.,1.7410417311

Be,0,0.,0.,-3.1208831432 HF $=-2045.0439894$ 


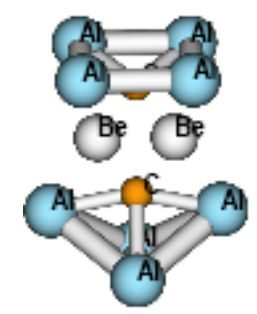

Al,0,2.1372912831,0.000016834,-1.6610894777

Al,0,0.0000099658,-1.2652778241,-3.1166018597 Al,0,-2.1372912831,-0.000016834,-1.6610894777 Al,0,-0.0000099658,1.2652778241,-3.1166018597 Al,0,1.3916387381,1.4815826068,2.2939917948 Al,0,-1.3916620768,1.4815606845,2.2939917948 Al,0,-1.3916387381,-1.4815826068,2.2939917948 Al,0,1.3916620768,-1.4815606845,2.2939917948 C,0,0.,0.,1.6893566035 C,0,0.,0.,-1.2233884358 Be,0,0.9737869731,0.0000076699,0.2670740543 Be,0,-0.9737869731,-0.0000076699,0.2670740543 HF=-2045.1227513

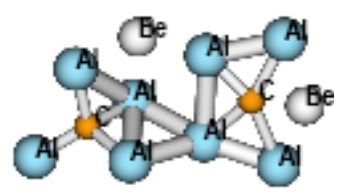

Al,0,2.7330398932,1.8332941962,-0.3660365037 Al,0,1.2189255371,0.2053827891,1.2961031963 Al,0,1.525021008,-1.2255861078,-1.2042462306 Al,0,4.5083922207,-0.8593892182,0.0865713693 Al,0,-2.9857773359,-2.3839936101,0.149470895 Al,0,-0.8034658316,-1.19860713,0.2514311615 Al,0,-1.3618600836,1.5179364186,-0.0503714583 Al,0,-3.8621632848,1.6464705367,-0.1857096045 C,0,2.7016081497,-0.09964491,-0.093960447 C,0,-2.5211791508,-0.1120139572,-0.0912145867 Be,0,0.698937899,2.36675666,0.5588695064 Be,0,-4.1289457978,-0.5396689515,-0.2070486367 HF $=-2045.1037361$

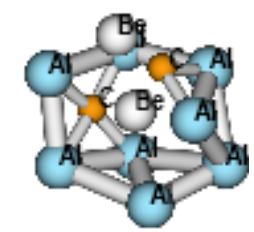

Al,0,-2.636296395,0.3913463238,-0.7468879372 Al,0,-1.291191776,2.1831210236,0.4999200998 Al,0,0.1108712866,1.1634798999,-1.669215637 
Al,0,1.648584919,2.1046629896,0.4042299245

Al,0,-1.2150168942,-1.9999245739,-0.7168402972

Al,0,-1.8641212003,-0.6141722198,1.747706639

Al,0,1.479284472,-1.649382287,-1.1804805517

C,0,1.5448628501,0.2903253366,-0.5999649346

Al,0,2.7753689728,-0.2779370206,0.8968556741

C,0,-0.3167213844,-1.6431388058,1.1640023998

Be,0,0.1375269606,-0.1474245487,0.4079834838

Be,0,1.2459398398,-2.052236188,1.2312745967

HF $=-2045.1675589$

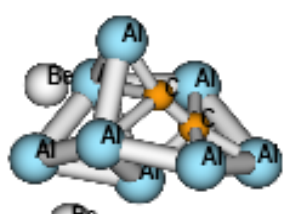

(B)

Be,0,-0.6864064819,-0.0000054036,-2.7625835662 Al,0,1.5926582509,0.0000125515,-2.4765882966 Al,0,-0.1391022614,-1.7110390697,-1.2018902049 Al,0,-2.4729212239,-0.000019481,-1.1798721471 Al,0,-0.139129222,1.7110368814,-1.2018901996 Al,0,-0.4212083856,2.2126456086,1.4463643155 Al,0,0.3060758356,0.0000024064,3.2621334709 Al,0,-0.4211735211,-2.2126522497,1.4463643088 $\mathrm{Al}, 0,1.5309883,0.0000120613,0.3543895694$ C,0,-0.3581809214,-0.000002824,1.3633232371 C,0,-1.0642068122,-0.0000083846,0.1850886773 Be,0,3.3523778215,0.0000264131,-1.0193194582 HF $=-$ 2045.1066649

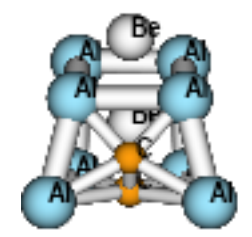

Be, $0,-0.3990151371,0.3990088511,-2.4753657683$ Al,0,1.8480483245,0.0304325669,-2.0214037802 $\mathrm{Al}, 0,-0.0304034526,-1.8480488037,-2.0214037802$ Al,0,-1.9734759397,-0.002960045,-0.8067175337 $\mathrm{Al}, 0,0.0029289548,1.9734759861,-0.8067175337$ Al,0,0.6130835611,2.2888821081,1.8668174967 Al,0,-2.2888724493,-0.6131196202,1.8668174967 Al,0,-0.0533969047,-1.9198270896,0.9173718644 $\mathrm{Al}, 0,1.9198262482,0.0534271498,0.9173718644$ C,0,-0.0916671374,0.0916656932,1.4677364194 C,0,-0.8935588924,0.8935448153,0.7346066814 
Be,0,1.7542045694,-1.7541769337,-0.5425911899 HF $=$-2045.114126

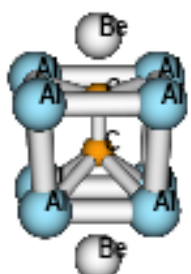

Be,0,0.,0.,-2.9579239622

Al,0,1.9404448747,0.0000152823,-1.4546933059 Al,0,0.0000152823,-1.9404448747,-1.4546933059 Al,0,-1.9404448747,-0.0000152823,-1.4546933059 Al,0,-0.0000152823,1.9404448747,-1.4546933059 Al,0,-0.0000172271,2.1873835677,1.3575057312 Al,0,-2.1873835677,-0.0000172271,1.3575057312 Al,0,0.0000172271,-2.1873835677,1.3575057312 $\mathrm{Al}, 0,2.1873835677,0.0000172271,1.3575057312$ C,0,0.,0.,1.156850991 C, $0,0 ., 0 .,-0.2081757079$ Be,0,0.,0.,2.7983495093 HF $=-\mathbf{2 0 4 5 . 0 7 4 7 9 5 2}$

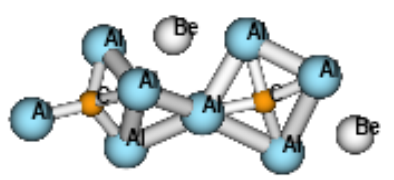

Al,0,-1.9011797727,1.8297042906,0.3015985217 Al,0,-0.5046318098,-0.4917688793,-0.1633101365 Al,0,-4.1969024931,0.7550153816,-0.379784079 Al,0,-3.0105884639,-1.8991941708,0.3912470253 Al,0,1.8931395324,-1.7084349891,0.4847791115 Al,0,1.5063166859,0.5653826271,-1.2625906659 $\mathrm{Al}, 0,2.5806082474,1.4155687943,1.0304055733$ Al,0,4.8775020603,-0.5591982868,-0.4670162582 C,0,-2.4452978022,-0.0983078488,-0.0651556564 C,0,3.0063327909,-0.1420235202,-0.1126092381 Be,0,0.4108103073,1.9404170017,0.3422095492 Be,0,-5.2962207466,-1.2779129431,0.1346182429 HF $=-\mathbf{2 0 4 5 . 0 8 4 5 1 9 7}$

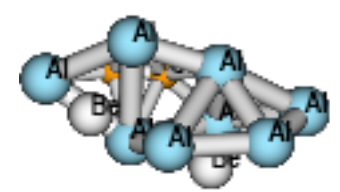

Al,0,0.8566641384,-1.3946346796,0.3507849154 Al,0,-0.7785046851,-1.2838420314,-1.7632469106 
Al,0,1.5771810683,0.9309781535,-1.3539194034 Al,0,-2.9022124737,0.055019306,-0.6789197125 Al,0,-0.4579429553,0.271515263,2.3501014481 Al,0,-0.6363640621,1.3221420591,-0.2546207212 Al,0,-2.7526609819,1.3884974171,1.5164419992 Al,0,3.5045354056,-0.9110475865,-0.5106492359 C,0,1.1093805168,0.6784494662,1.0330715948 C,0,2.2496906058,0.1640678711,0.6122320656 Be,0,-1.3940753607,-1.1076246131,0.5963246524 Be,0,1.5207084503,-1.3866920719,-1.9461903747 HF $=-$ 2045.1264759

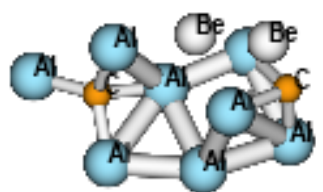

Al,0,-1.9379615486,-1.6697561153,0.6367287518 Al,0,-0.4102735734,0.4462149837,0.71298513 Al,0,-1.9866529003,1.214858358,-1.3979498274 Al,0,-4.1433292044,0.5699338893,1.0140454619 Al,0,3.1856001236,1.0638223348,-0.5974909173 Al,0,1.9144827944,-0.0235304538,1.6702765571 Al,0,1.4337986265,-1.317923659,-1.1666850525 Al,0,0.7348470966,1.1391277434,-1.5669673448 C,0,-2.3412452834,0.2716120822,0.3050514908 C,0,3.0618181548,-0.7318435352,0.0129702248 Be,0,0.3943017616,-1.8886360364,1.0228717666 Be,0,2.4556768347,-2.0449447981,0.7590316941 HF $=-2045.1413915$

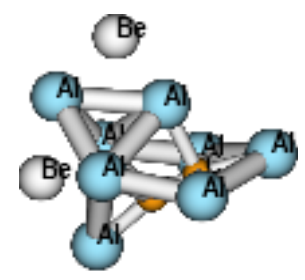

Be,0,-0.6864064819,-0.0000054036,-2.7625835662 Al,0,1.5926582509,0.0000125515,-2.4765882966 Al,0,-0.1391022614,-1.7110390697,-1.2018902049 Al,0,-2.4729212239,-0.000019481,-1.1798721471 Al,0,-0.139129222,1.7110368814,-1.2018901996 Al,0,-0.4212083856,2.2126456086,1.4463643155 Al,0,0.3060758356,0.0000024064,3.2621334709 Al,0,-0.4211735211,-2.2126522497,1.4463643088 Al,0,1.5309883,0.0000120613,0.3543895694 C,0,-0.3581809214,-0.000002824,1.3633232371 
C,0,-1.0642068122,-0.0000083846,0.1850886773

Be,0,3.3523778215,0.0000264131,-1.0193194582

\section{HF=-2045.1066649}

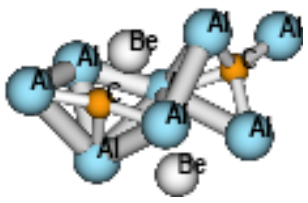

Al,0,-2.2286511706,1.6905455021,-0.1853250684 Al,0,0.5758040523,1.3927813631,1.2548145173 Al,0,-1.3385278244,-0.9202923078,1.5591209892 Al,0,-4.0812363216,-1.1046404099,-0.393483663 Al,0,1.7280594341,-1.6242970848,-1.0366261766 Al,0,-0.5366171399,-0.2994262067,-0.9245466283 Al,0,1.9479223849,1.1707798258,-1.1009015394 Al,0,3.8109995039,-0.6481297294,0.4100218606 C,0,2.059959522,0.2219231746,0.7479829043 C,0,-2.3382346406,-0.2781265537,-0.0090712029 Be,0,0.9257896925,-0.9615472178,1.1656697557 Be,0,-0.1110740004,2.1595591911,-0.9190287547 HF $=-2045.1482644$

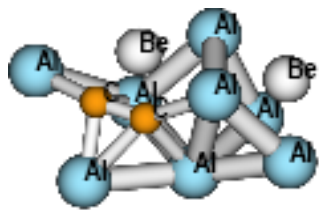

Al,0,0.6324094968,-1.2145041956,-0.8314432578 $\mathrm{Al}, 0,3.0958078844,-1.2635022245,0.147745206$ Al,0,-2.0950673332,-2.0131366359,-0.5124340446 Al,0,1.9884642066,1.0777695406,-1.5773073797 Al,0,-1.1470058753,0.9243335577,-1.1382683624 Al,0,1.0787378107,-0.4746758516,1.7904567912 Al,0,0.7877709515,2.1068452912,0.6107856171 Al,0,-3.6664424337,0.7442433384,0.2527551069 C,0,-0.6620681424,-0.6923428027,0.7028908833 C,0,-1.9862466714,-0.2689353451,0.6364594046 Be,0,2.7873952314,0.9088286892,0.6734214168 Be,0,-1.0076158117,0.8991268667,1.4051117023 HF $=-\mathbf{2 0 4 5 . 1 4 4 9 3 5 2}$ 


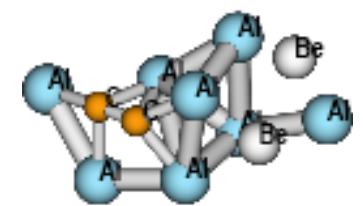

Al,0,0.7095452123,-1.6535613011,0.5193684163 Al,0,-3.5905012111,-1.5055714764,0.4469241541 Al,0,2.7922329512,-0.2837582956,1.2013041544 Al,0,-1.3942236638,-0.3016179061,1.4334429094 Al,0,0.2199612091,1.3157879997,0.1162970735 Al,0,-0.1114161787,-0.7625510401,-1.9396142599 Al,0,-1.9821808812,0.8744886247,-1.350797614 Al,0,3.1460323662,2.4180417441,0.2342320654 C,0,1.5075489887,-0.2258918348,-0.8991094025 C,0,2.2269297154,0.8466549485,-0.5455552059 Be,0,-3.4998780821,0.6800437336,0.3736865397 Be,0,-1.4175518368,-1.9402780391,-0.3554495491 HF $=-2045.1166349$

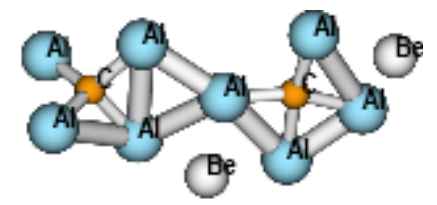

C,0,-2.5512687368,-0.0206239142,-0.1196882901 $\mathrm{Al}, 0,-3.2012112233,-1.8014187,0.2086971096$ Al,0,-0.6133389314,-0.0444045626,0.2069093445 Al,0,-2.2138752076,1.9750064117,-0.3753235133 Al,0,-4.3361429085,0.6432159599,-0.7511065196 Be,0,-0.0167879299,2.2698570135,0.0789797303 Al,0,4.4155015464,-0.8524389285,-1.2160241352 Al,0,1.8684407307,1.2053754884,-0.1905027059 Al,0,1.5599851774,-1.5943054866,0.1754297752 Al,0,3.9524509216,0.2745786548,1.8517721496 C,0,3.0778309473,-0.2931119754,0.1389476123 Be,0,-5.4264382283,-1.1674819001,0.1851138954 HF $=-2045.0713641$

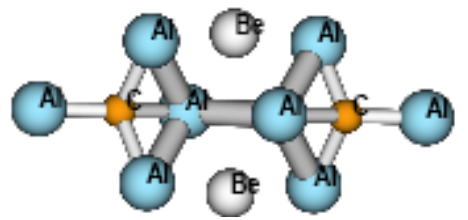

Al,0,-2.1032317178,-1.702504302,-0.9927590088 Al,0,-0.8800314323,-0.3909922806,0.9423545171 Al,0,-4.6695855079,-0.6262257001,0.6470116954 Al,0,-2.3944471494,1.6008301443,0.1057781575 Al,0,2.3932592337,-1.6007915317,-0.1042213197 Al,0,2.1018261237,1.7000432895,0.9947500262 
Al,0,0.8817978276,0.3920242673,-0.9444964224 $\mathrm{Al}, 0,4.6698466403,0.6273550563,-0.6482440746$ C,0,-2.7793229992,-0.3205124479,0.2271405441 C,0,2.7806423482,0.3202010728,-0.2259883861 Be,0,-0.1645321786,1.8658129051,0.6188920153 Be,0,0.1643925967,-1.864497407,-0.6211843568 HF $=-2045.1331639$

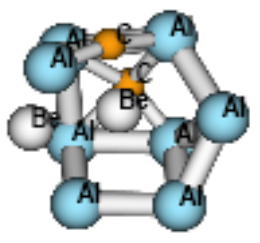

Al,0,2.5731604272,1.30866114,-0.1168441209 Al,0,2.1909631768,-0.9845374794,1.1243791384 Al,0,0.8663089487,0.1562136214,-1.9843033125 Al,0,0.4703046571,-2.1125791339,-0.6637888687 Al,0,0.4128633912,2.5317136863,-0.7765693395 Al,0,-1.4341411739,-0.2018461613,2.6614316223 Al,0,-1.895669603,1.1951645491,-0.8946731734 C,0,-0.8036946749,-0.5217058899,-1.2441975245 Al,0,-1.9200138781,-1.3992294592,0.1613045585 C,0,-1.8717603257,0.5193242879,0.9556146002 Be,0,0.0361986226,-1.7550640283,1.6327474001 Be,0,-0.1302879462,0.1545639518,0.3895833477 HF $=-\mathbf{2 0 4 5 . 1 3 5 4 0 8 8}$

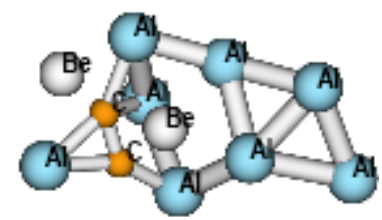

Al,0,-1.40951702,-2.4271595342,0.0802546502 Al,0,3.5038671973,-0.9106856283,0.0657658697 Al,0,-1.497911451,-0.0985658517,1.7799413344 Al,0,4.2827922134,1.6796417409,0.080435326 $\mathrm{Al}, 0,-3.8282120344,0.7367445223,-1.2742040749$ Al,0,0.9788928943,-1.5612493073,0.3646624179 Al,0,1.6166176044,0.8609354379,-0.5072973889 Al,0,-0.5150393211,2.0435862267,0.298073495 C,0,-2.2702945942,-0.441915378,-0.4115899746 C,0,-1.9417519058,0.9326454013,-0.4586016868 Be,0,-3.3026571342,-1.6736998742,-0.7435074357 Be,0,-0.5566158855,-0.1129498807,-0.8360078672 HF $=-\mathbf{2 0 4 5 . 1 3 1 1 3 3 5}$ 


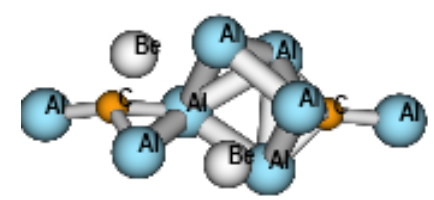

C,0,-2.5794607712,-0.0679969876,-0.0624799983 Al,0,0.4065239332,-1.715850877,1.2355542757 Al,0,-1.2674320503,-1.3844952959,-0.7162129689 Al,0,-1.253226317,1.4301969474,-0.3240454782 Al,0,0.8476629004,0.153392404,-1.1506363919 Al,0,2.2169146426,1.1955928611,1.0514323247 Al,0,-1.6622287532,-0.2180952708,1.7534780568 Al,0,4.3322484363,0.4894457206,-1.6837629107 Al,0,-4.4743970054,-0.018648569,-0.4887748089 C,0,2.7883861319,0.1507504055,-0.5214928519 Be,0,0.019023055,1.3369909129,1.6334766116 Be,0,2.4428750971,-1.2386192804,0.2921283434 $\mathrm{HF}=-\mathbf{2 0 4 5 . 1 6 1 9 3 8 8}$

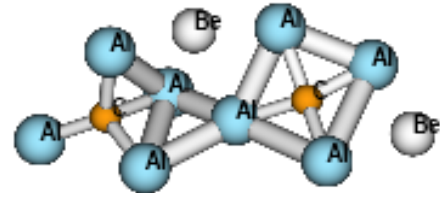

C,0,2.9950717944,0.274411408,-0.1552558724 Al,0,4.8451104654,0.8816418927,-0.2526012255 Al,0,1.4195147726,0.4333918145,-1.4061674402 Al,0,2.6232792917,-1.6648629228,-0.2734880472 Al,0,1.9385122774,1.0463797205,1.3736641805 Be,0,0.4096771669,-1.6869433435,-1.0191419534 Al,0,-2.9594887324,1.098385423,1.6900613888 Al,0,-1.8994661539,-1.647506892,-0.8566544899 Al,0,-4.2203455888,-0.4592887459,-0.5528476031 Al,0,-0.508272458,0.4634786569,0.2225241443 C,0,-2.4435018225,0.0399697394,0.1459415302 Be,0,-5.2632747147,0.7226100451,1.2135180166 $H F=-2045.0845196$

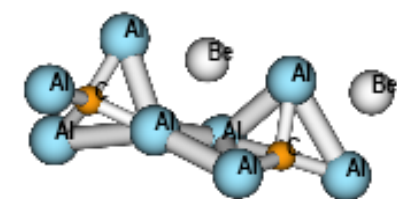

C,0,2.8632867632,0.2268634914,0.0907107689 Al,0,3.4726206398,0.3991707475,-1.7970553771 Al,0,2.259234111,-1.5172574847,0.8492754991 Al,0,0.9419787819,0.6213416909,-0.0687606209 Al,0,4.0618342542,1.1429658574,1.3675256266 Be,0,-0.0817047926,-1.5269938698,0.7068211164 
Al,0,-1.0080824089,-0.4633040339,-1.2411969092 Al,0,-2.4376013701,-1.3907568342,0.8342899762 Al,0,-1.4729414235,1.8111393628,0.4657144467 Al,0,-4.4690868807,0.2020951479,-0.6563995716 C,0,-2.6075511564,0.3386672813,-0.2751910144 Be,0,-4.6827546548,-1.9388342635,0.3713717749 HF $=-2045.0860319$

(A)

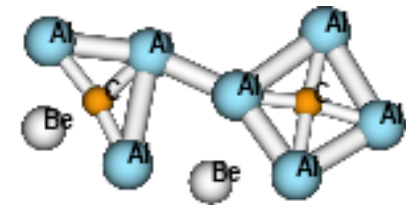

C,0,-3.2348494331,-0.2442948314,0.2979704375 Al,0,-3.4969900561,-1.3431211771,1.964049853 Al,0,-1.3339806215,-0.2174381125,0.2990730061 Al,0,-3.1351662429,0.8575399264,-1.375934594 Al,0,-5.2422917422,-0.0599964772,-0.0271981944 Be,0,-0.8683306647,1.1251184083,-1.7319945188 Al,0,1.1569582419,-0.6698792302,1.0427132951 Al,0,1.434741814,1.0289799971,-1.5332621574 Al,0,6.2582277857,0.5645700025,-0.708334756 Al,0,3.8514540697,-0.6593943292,1.087188084 C,0,2.3982185399,0.143411581,-0.1660644898 Be,0,3.7711789466,0.6471095179,-0.8978216459 HF $=-$ 2045.102178

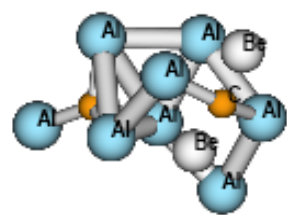

Al,0,-1.0208486023,0.7074460412,0.5776403681 Al,0,-0.8899665851,3.0053479988,-0.6477767853 Al,0,-0.1248131819,-2.1626616572,1.3300168527 Al,0,-3.4342258286,-1.6659626471,0.6029884632 Al,0,1.3949504636,0.1672166576,1.764018571 Al,0,1.6108358514,2.3322597759,-0.0881983714 Al,0,-0.764179062,-1.0926683323,-1.4960660793 Al,0,1.9095930728,-1.3834622655,-1.2229934707 C,0,2.0283758775,0.5704144378,-1.0706408129 C,0,-1.5428410773,-1.2072690955,0.2783632007 Be,0,3.0318644989,0.403649209,0.1903623906 Be,0,0.5254583851,0.852207171,-1.6657420039

$$
\text { HF }=-2045.1515559
$$

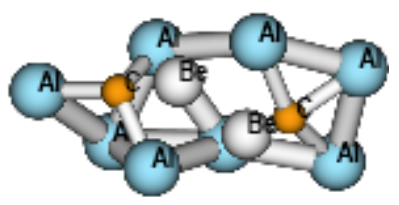


C,0,-1.977988473,0.4386417735,-0.2635075821

Al,0,0.9735224058,-1.2942244431,0.9940714522

Al,0,-0.4234854251,-0.2529199825,-1.3254512589

$\mathrm{Al}, 0,-3.0340834102,0.0791975785,-2.0090060372$

Al,0,1.4955330427,1.5800600613,-0.4449058604

Al,0,3.965973219,-0.1492641754,0.9824928688

Al,0,-1.4256960231,-0.1626710811,1.6420914408

Al,0,2.2562883555,-0.8854289108,-1.3344728666

Al,0,-3.7882361894,-0.1000840318,0.5392710277

C,0,2.0788806594,0.3916996308,1.0836620998

Be,0,-0.8531851767,1.5839813672,0.2428158588

Be,0,0.6374449776,1.0228452276,1.6336573738

$\mathbf{H F}=-\mathbf{2 0 4 5 . 1 8 1 8 3 7 7}$

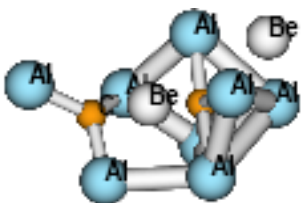

Al,0,-3.90306115,-0.8823272771,-0.6341784854 $\mathrm{Al}, 0,0.9497178083,0.3955174453,2.0137098427$ Al,0,-2.042679879,1.9035188444,0.0462451535 Al,0,0.8712781407,2.0986772571,-0.1910814272 Al,0,-1.1230363452,-1.0560377046,1.2218091737 Al,0,2.897905923,-0.1365104365,0.48563005 Al,0,0.8329558647,-2.15283588,-0.2030681581 C,0,-2.2364810756,-0.0308148001,-0.0715889411 Al,0,1.5051957466,0.3505176295,-2.0492850598 C,0,0.85226898,-0.1403376004,-0.0162431967 Be,0,2.7732633327,-1.4695717068,-1.3289854041 Be,0,-0.6588425438,0.0346107037,-0.7810549302 HF=-2045.160281

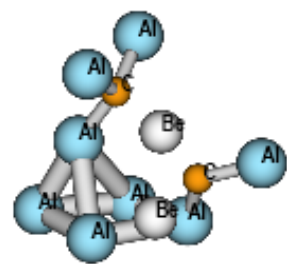

Al,0,-2.1952195402,2.4168299691,-0.8097392164 Al,0,-2.2589411042,-0.0949122622,-1.6702209975 Al,0,-1.8192614411,0.9729031561,1.2784310685 Al,0,-1.644541444,-1.6258787013,0.3300380971 Al,0,0.1126840119,1.4624063772,-0.652976073 Al,0,2.6357978227,-0.0135808594,-2.1878082456 Al,0,1.041043884,-3.4831647964,1.7130111775 Al,0,3.0384785984,1.3892506343,0.8526519106 Be,0,1.1133112608,-0.7413392069,0.3831664086 
C,0,1.8111271197,0.5353174639,-0.4656467535

C,0,0.1886389727,-1.7999572275,1.2336901965

Be,0,-0.5705929589,-0.689225079,2.1912583327

HF $=$-2045.178811 


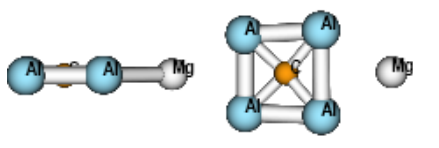

Mg,0,0.4859931566,-4.6103377435,5.2976459433 Al,0,1.3694839647,-2.1326774538,4.0694487086 Al,0,-0.7404229838,-3.8313990858,2.7842674575 Al,0,1.0922712387,-0.4388283642,1.9835558657 Al,0,-0.8353449928,-1.9907075628,0.8093339751 C,0,0.228548696,-2.1655655097,2.4887869186 Mg,0,-0.0492539743,0.4756769312,-0.5453335918 C,0,-0.3134054373,2.9686239261,-3.4118526506 Al,0,0.7730798488,1.3663397565,-3.0755269727 Al,0,-1.4559851951,4.5944068212,-3.6871792661 Al,0,-1.2085892395,2.7675708075,-1.674295917 Al,0,0.6415281483,3.1112627159,-5.1703233762

\section{HF $=-\mathbf{2 4 1 5 . 8 1 0 9 2 0 1}$}

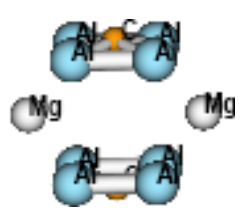

Al,0,1.9359918483,-0.0577630138,-2.0362688017 Al,0,0.0577630138,-1.9359918483,-2.0362688017 Al,0,-1.9359918483,0.0577630138,-2.0362688017 Al,0,-0.0577630138,1.9359918483,-2.0362688017 Al,0,-0.0577630138,1.9359918483,2.0362688017 $\mathrm{Al}, 0,-1.9359918483,0.0577630138,2.0362688017$ Al,0,0.0577630138,-1.9359918483,2.0362688017 Al,0,1.9359918483,-0.0577630138,2.0362688017 C,0,0.,0.,2.416634562 C,0,0.,0.,-2.416634562 Mg,0,1.9831113038,1.9831113038,0. Mg,0,-1.9831113038,-1.9831113038,0.

\section{HF $=-\mathbf{2 4 1 5 . 8 3 4 7 6 5 8}$}

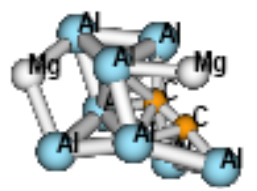

Al,0,0.8035566714,-1.1677202341,-0.7616920715 Al,0,2.8577038078,-0.899461938,1.0361448406 Al,0,-1.8817588951,-1.8501394732,-1.0169755229 Al,0,1.1239633452,1.0162865394,-2.5881274234 Al,0,-0.9775279389,1.1164472659,-0.9967688653 Al,0,0.4047781668,-1.2260445513,2.2113005634 Al,0,0.9620805942,1.2535917335,0.8702686435 
Al,0,-3.8061425579,0.3549873755,-0.2331469493

C,0,-0.6482288155,-0.5084908854,0.4692209186

C,0,-2.0125040962,-0.2047770687,0.2923161272

Mg,0,3.3411558199,0.8757291617,-0.8871387739

Mg,0,-1.4546636569,0.9997958712,2.108616768

HF $=-\mathbf{2 4 1 5 . 8 4 9 1 4 9 4}$

Mg,0,-1.1854917132,-0.0000093351,-3.1197183013

$\mathrm{Al}, 0,1.44842894,0.0000114148,-2.2126579091$

Al,0,-0.3101893624,-1.7511699368,-0.9455240267

Al,0,-2.7894102143,-0.0000219751,-0.7800997458

Al,0,-0.3102169555,1.7511650519,-0.9455240213

Al,0,-0.539731852,2.159322617,1.7198129116

Al,0,0.1495619592,0.000001173,3.4862098668

Al,0,-0.5396978276,-2.1593311266,1.719812905

Al,0,1.28573182,0.0000101287,0.6098374469

C,0,-0.6179941255,-0.0000048714,1.6383401549

C,0,-1.1844781971,-0.0000093325,0.3843882126

Mg,0,3.8260449913,0.0000301446,-0.7645022621

HF $=-2415.847859$

C,0,-2.2775290068,0.276136709,0.2045859036

Al,0,-3.2521010614,-1.2110239433,1.1188075993

Al,0,-0.3668084088,-0.1326288469,0.0084795929

Al,0,-1.9724424068,1.900467801,1.2964670631

Al,0,-2.9871324214,0.5022261969,-1.6505041704 Mg,0,1.0030982706,2.2004284243,1.2017227155

Al,0,4.5751188567,-1.4769179801,-1.0294506005

Al,0,1.7573395637,0.752780183,-1.1536790285

Al,0,1.7685076838,-1.6286349315,0.5695788782

Al,0,3.6697440172,1.0298654868,1.007958984

C,0,3.1373955203,-0.3937870385,-0.2410477831

Mg,0,-4.8912761688,-1.8557483894,-1.3651216204

HF $=-\mathbf{2 4 1 5 . 8 3 9 7 9 0 5}$

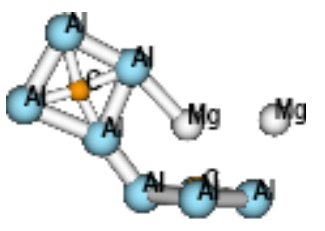


C,0,-2.2103399907,0.7405902071,0.

Al,0,-1.6657677951,-1.1021620691,0.

Al,0,-2.5077829801,0.6350993231,2.0269434718

$\mathrm{Al}, 0,-3.2668998239,2.4552607113,0$.

Al,0,-2.5077829801,0.6350993231,-2.0269434718

$\mathrm{Al}, 0,2.617394518,0.1424786338,0$.

$\mathrm{Al}, 0,2.7413456909,-3.8691816708,0$.

C,0,2.5544785268,-1.8843348214,0.

Al,0,0.6812441535,-1.744585504,0.

$\mathrm{Al}, 0,4.5910066169,-1.6235158391,0$.

Mg,0,0.0064958061,1.2370420992,0.

Mg,0,-0.9182189242,4.1789628907,0.

HF $=-2415.8088606$

Al,0,-0.6189340788,-1.9689171534,0.1372886103 Al,0,3.4878568712,-0.650239403,-1.0317662338 Al,0,-3.0127451991,-1.388219568,-0.7535129345 Al,0,0.9401544003,-0.468732303,-1.4579656487 Al,0,-0.9418457691,1.0848153178,-0.543215932 $\mathrm{Al}, 0,-0.1378661888,-0.0652388071,2.1393923868$ Al,0,1.226175562,1.7274946024,0.8419409949 Al,0,-4.1163323067,1.3545577923,-0.5694086663 C,0,-1.7373153101,-0.3594588043,0.9828043193 C,0,-2.7069937934,0.3839406714,0.4007831719 Mg,0,3.6226211392,1.9239490783,-0.4567819337 Mg,0,2.037531514,-1.5305038629,1.1053395632 HF $=-\mathbf{2 4 1 5 . 8 3 9 6 7 2 7}$

CMg

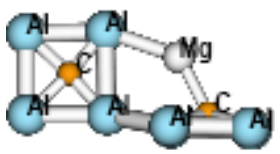

Al,0,-3.2174141257,-2.6423793275,0.

Al,0,-0.7183519401,-3.9087930849,0.

$\mathrm{Al}, 0,-2.0761459474,-0.1813354561,0$.

Al,0,0.5515804712,-1.45177301,0.

C,0,-1.2989952226,-1.9579925739,0.

C,0,2.0860449507,1.6954741438,0.

$\mathrm{Al}, 0,1.9182581424,0.3153152075,1.4740522692$

Al,0,3.2153606959,2.7472652293,-1.3733752743

Al,0,1.9182581424,0.3153152075,-1.4740522692

Al,0,3.2153606959,2.7472652293,1.3733752743 
Mg,0,-5.4924175008,0.6804557598,0.

Mg,0,-0.1085890091,1.681516794,0.

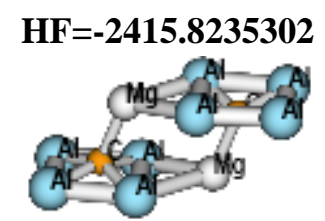

C, $0,0 ., 0 ., 0$.

C,0,0.,0.,4.68311269

Mg,0,1.6026321569,0.,1.474666741

Mg,0,-1.6026321569,0.,3.208445949

Al,0,0.1534361555,1.3646447315,-1.5591193908

Al,0,0.1534361555,-1.3646447315,-1.5591193908

Al,0,-0.9579437757,1.5027307829,0.9270552956

Al,0,-0.9579437757,-1.5027307829,0.9270552956

Al,0,-0.1534361555,1.3646447315,6.2422320808

Al,0,-0.1534361555,-1.3646447315,6.2422320808 Al,0,0.9579437757,1.5027307829,3.7560573944

Al,0,0.9579437757,-1.5027307829,3.7560573944

HF $=-\mathbf{2 4 1 5 . 8 4 8 5 8 7 7}$

CAg

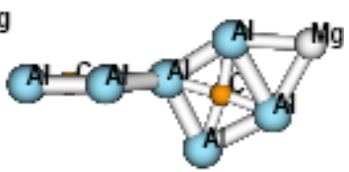

Al,0,-0.7131885975,4.6727978956,0.

$\mathrm{Al}, 0,-2.1947962081,2.3531874111,0$.

Al,0,1.7595367486,2.9372719563,0.

Al,0,0.1413243811,0.8512194336,0.

C,0,-0.1990327161,2.81346533,0.

C,0,-0.3711284071,-2.5979526603,0.

Al,0,-0.2849035432,-1.292164808,1.4286722941

Al,0,-0.5504618849,-4.0401509057,-1.3899940682

Al,0,-0.2849035432,-1.292164808,-1.4286722941

Al,0,-0.5504618849,-4.0401509057,1.3899940682

Mg,0,1.8998556806,5.7645910763,0.

Mg,0,1.2862339574,-6.0346797861,0.

HF $=-\mathbf{2 4 1 5 . 8 1 5 9 5 3 7}$

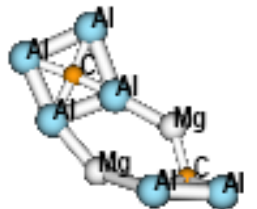

Al,0,-2.816390986,-1.60479028,0.0525087917

Al,0,-1.5298098861,0.8141197147,-0.0297180228

$\mathrm{Al}, 0,-5.2566734241,-0.4294272378,0.0093584811$ 
Al,0,-4.0536722995,2.1858571125,-0.0797346124 Al,0,4.3068369836,0.753874391,1.3540902941 Al,0,2.1795035578,-0.9873415792,1.4958673917 Al,0,2.1825393589,-1.0880641103,-1.4196254328 Al,0,4.3096984113,0.6589376967,-1.3939269483 C,0,-3.3454391959,0.303142227,-0.0139553203 C,0,2.8341679679,0.2127431181,-0.0043985172 Mg,0,-0.0733392212,-1.9638713083,0.0677702709 Mg,0,1.0634404763,1.3774991192,-0.0464816228 HF $=-\mathbf{2 4 1 5 . 8 4 1 2 7 8}$

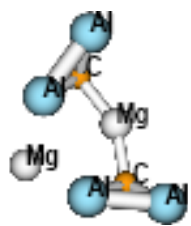

Mg,0,-0.6161726532,0.6161629451,-0.0779554338 Al,0,1.8968981857,0.1933135175,-1.8257121716 Al,0,-0.1932836306,-1.8969012313,-1.8257121716 Al,0,-1.7136002678,-0.2253871423,-3.3676579496 Al,0,0.2253601433,1.7136038186,-3.3676579496 Al,0,-0.185027304,2.1229159772,3.1325245671 Al,0,-2.1229188921,0.1849938561,3.1325245672 Al,0,-0.438394553,-1.6534216035,2.0058242389 $\mathrm{Al}, 0,1.6534146961,0.4384206037,2.0058242389$ C,0,-0.3872978825,0.3872917805,1.9986894128 C,0,-0.1297928378,0.1297907928,-2.062568438 Mg,0,1.8253989375,-1.8253701774,0.2291077958 HF $=-2415.834242$
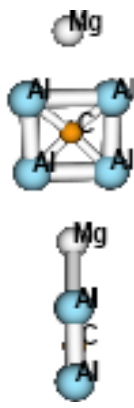

Al,0,-0.0698708835,-4.7946441238,0. $\mathrm{Al}, 0,0.6946633839,-2.2030592696,0$. Al,0,-2.8984276141,-3.813965837,0. Al,0,-1.8940427304,-1.3044668285,0. C,0,-1.0749791443,-3.1236585878,0. C,0,1.4754204359,4.2900141163,0. Al,0,1.0308294321,2.9821499334,1.3990453731 Al,0,1.9082801948,5.5644124836,-1.4864882524 Al,0,1.0308294321,2.9821499334,-1.3990453731 
$\mathrm{Al}, 0,1.9082801948,5.5644124836,1.4864882524$

Mg,0,-2.2994103606,-6.6558786225,0.

Mg,0,0.2461031875,0.6809630185,0.

HF $=-\mathbf{2 4 1 5 . 8 1 0 9 1 6 8}$

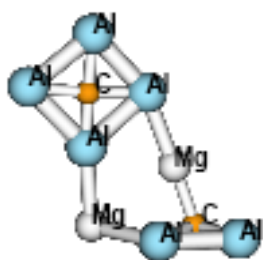

Al,0,-4.6341986822,-1.8896049122,0.

Al,0,-2.6831481895,-3.9695197711,0.

$\mathrm{Al}, 0,-2.7086954548,0.0049576043,0$.

Al, $0,-0.6871643174,-1.9315101805,0$.

C,0,-2.5836914205,-1.9548466364,0.

C,0,1.8076309919,1.9014058629,0.

Al,0,2.4645595673,0.7201272127,1.492132479

Al,0,2.2262032056,3.4262362981,-1.356520053

Al,0,2.4645595673,0.7201272127,-1.492132479

Al,0,2.2262032056,3.4262362981,1.356520053

Mg,0,2.0334918895,-1.6078455468,0.

Mg,0,-0.2028071524,1.0852620245,0.

\section{HF $=-\mathbf{2 4 1 5 . 8 4 5 6 3 5 2}$}

Al,0,2.6358511569,-1.8553353047,-0.5349897506 Al,0,2.8504746111,0.5325579941,-1.6535470698 Al,0,1.6924962726,0.3618473074,0.8271337038 Al,0,-0.0259946164,1.9256777732,2.1182422844 Al,0,0.6302827071,-2.0089775641,1.2922820228 Al,0,-3.9212629972,-0.6690226978,-1.6063302055 Al,0,-1.7340164371,-1.2844667844,1.0367309335 C,0,-0.1687380053,-0.0142426795,1.4383997063 Al,0,-1.5713336958,1.3061425155,0.1611087245 C,0,-2.3312247124,-0.334974226,-0.5722619366 Mg,0,0.8249850838,2.5881634186,-0.8417342198 Mg,0,-0.1778754763,-0.581013475,-1.3686845284 HF $=-\mathbf{2 4 1 5 . 8 3 5 2 1 2 6}$

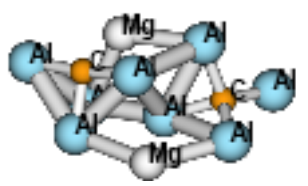

Al,0,-2.74036404,1.6243724397,0.1100531733 
Al,0,0.5617848359,0.5927045446,1.6860171998 Al,0,-1.5904875233,-1.3514461119,1.2972063746 Al,0,-3.9938713169,-1.3542553063,-0.7564610536 Al,0,1.7322662791,-0.8000650933,-1.7971011335 Al,0,-0.6349244889,-0.008218689,-0.9651761091 $\mathrm{Al}, 0,2.1860329943,1.5245201808,-0.4646368703$ Al,0,3.8455013022,-0.7923120774,0.1036979984 C,0,2.1453410689,-0.0640506552,0.8101208636 C,0,-2.433417461,-0.2805075476,-0.1831200379 Mg,0,1.0294940668,-1.9988457188,0.5707604997 Mg,0,-0.1985554166,2.7828832758,-0.0323271239 HF $=-\mathbf{2 4 1 5 . 8 4 1 5 1 4 1}$

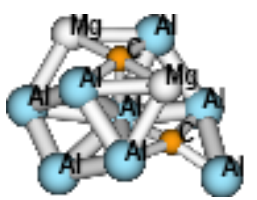

Al,0,0.4102453928,-2.1012473166,0.5816747739 Al,0,-1.546450405,-0.3265760267,1.4302839863 Al,0,2.4420043779,1.0336556628,-0.9139426093 Al,0,3.5661312335,-1.4725326228,-0.3699262754 Al,0,-3.0853560182,-0.257091357,-0.8580048845 Al,0,-0.1094377237,0.0922549391,-0.8663453805 Al,0,-1.6110949733,-2.3032252845,-1.0275595676 Al,0,0.4064732589,2.789230176,-0.0034454927 C,0,-0.4993567736,1.290341105,0.8938065541 C,0,1.729112549,-0.74737197,-0.0989196394 Mg,0,-2.2955925423,2.1988891287,0.0272051719 Mg,0,1.1688232499,0.287285786,1.771555608 HF $=-\mathbf{2 4 1 5 . 8 3 5 3 1 6}$ 


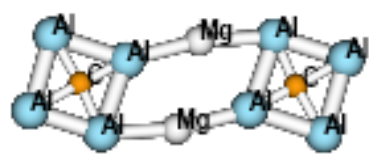

Al,0,0.8254465151,5.7263403594,0.3950447504 Al,0,-1.7395314854,4.9275901239,-0.5152514727 Al,0,1.6295414915,3.1620766593,0.6296343486 Al,0,-0.8892008024,2.2286022623,-0.2665155567 C,0,-0.0849494732,3.9082931198,0.0445370036 C,0,0.0855793914,-3.9073376486,-0.0478899391 Al,0,-1.6285512729,-3.1605614641,-0.6303087108 Al,0,0.8931163988,-2.2303161363,0.2693855016 Al,0,-0.8234373341,-5.7248509714,-0.4034458463 Al,0,1.7355556553,-4.9295705798,0.5222925385 Mg,0,-1.5232688515,-0.4318058045,-0.5397990225 Mg,0,1.5197697961,0.4320752945,0.5405703084 $H F=-2415.842215$

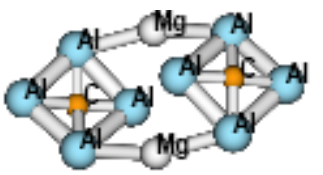

Al,0,-2.5940852992,-2.0974404897,-0.4137268401 Al,0,-0.9902024302,-0.4767067122,1.170791231 Al,0,-4.627220195,-0.3174428187,-0.2715145793 Al,0,-2.9102705241,1.4906282735,0.779793733 Al,0,2.9102684245,-1.4906353005,-0.7797851622 Al,0,2.5940843501,2.0974439206,0.4137399858 Al,0,0.9902402828,0.4767171784,-1.1708164637 Al,0,4.6272023838,0.3174101803,0.2715951738 C,0,-2.8446636959,-0.4589195771,0.6260533763 C,0,2.8446806856,0.4589164259,-0.6260308194 Mg,0,-0.1969292789,2.2346183089,0.7432638289 Mg,0,0.1969023752,-2.2345888176,-0.7433586089 $H F=-2415.8487963$

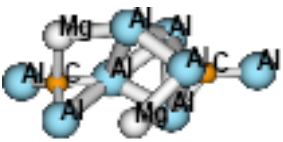

C,0,-2.7063789963,-0.3446830973,-0.3372035893 Al,0,-0.1991364016,-0.0412565803,2.2149597822 Al,0,-1.4065905927,-1.5843068205,0.5442801844 Al,0,-1.3382938039,0.2418861316,-1.6553547423 Al,0,0.8331689332,-0.710677171,-0.6207256973 Al,0,2.5753171969,1.6149320963,-0.6696985598 Al,0,-2.2056505892,1.111429195,0.9936262586 Al,0,4.1570921724,-1.4320446443,-1.0451060397 
Al,0,-4.5221693663,-0.8830903523,-0.7891246599

C,0,2.7481635826,-0.2878268074,-0.3440718161

Mg,0,-0.1997133774,2.4588091609,-0.3788368628

Mg,0,2.4606054063,-0.3191653842,1.8322133288

HF $=-\mathbf{2 4 1 5 . 8 6 4 3 6 9}$ 


\section{${ }^{a}$}

Al,0,1.9450675911,-0.0570475778,-2.2611169374 Al,0,0.0570475778,-1.9450675911,-2.2611169374 Al,0,-1.9450675911,0.0570475778,-2.2611169374 Al,0,-0.0570475778,1.9450675911,-2.2611169374 Al,0,-0.0570475778,1.9450675911,2.2611169374 Al,0,-1.9450675911,0.0570475778,2.2611169374 Al,0,0.0570475778,-1.9450675911,2.2611169374 Al,0,1.9450675911,-0.0570475778,2.2611169374 C,0,0.,0.,2.5869627517 C,0,0.,0.,-2.5869627517 Сa, $0,2.2506446187,2.2506446187,0$. Ca,0,-2.2506446187,-2.2506446187,0.

\section{HF $=-3370.7854905$}

Ca, 0,-1.6594508091,3.1357465001,-1.0098138998 Al,0,0.9638750036,2.8245406308,0.5865623489 Al,0,1.0727475428,0.6567644294,-1.0067530739 Al,0,-2.1151973052,-0.1389966476,-1.2871659443 Al,0,-0.4012152006,0.6567528088,1.4154083047 Al,0,-1.9330388037,-1.4998751693,1.1571786592 Al,0,-0.6314593508,-4.0411882619,-0.384282619 Al,0,0.1394985442,-1.4998588295,-2.2486197182 $\mathrm{Al}, 0,1.2584558832,-1.8047886572,0.7658020352$ C,0,-0.52892159,-2.095082502,-0.3218757722 C,0,-0.3476066274,-0.6686984069,-0.2115329694 Ca, $0,2.9925261705,0.8437100754,1.8210520271$ HF $=-3370.7861739$

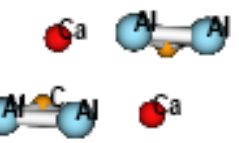

C, $0,0.0486956084,0 ., 0.1184436681$

C, $0,-0.0486956084,0 ., 5.2352669019$

Ca,0,2.044997002,0.,1.742726755

Ca,0,-2.044997002,0.,3.610983815

Al,0,0.3942077123,1.3646426786,-1.3970612071 Al,0,0.3942077123,-1.3646426786,-1.3970612071 
Al,0,-0.9676184332,1.4929516233,0.9696173247

Al,0,-0.9676184332,-1.4929516233,0.9696173247

Al,0,-0.3942077123,1.3646426786,6.7507717771

Al,0,-0.3942077123,-1.3646426786,6.7507717771

Al,0,0.9676184332,1.4929516233,4.3840932453

Al,0,0.9676184332,-1.4929516233,4.3840932453

\section{HF $=-3370.7820395$}

c
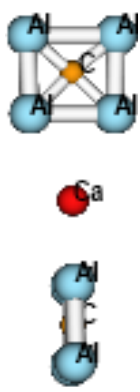

$\mathrm{Al}, 0,-0.0872827123,-4.7805806334,0$.

$\mathrm{Al}, 0,0.7133687203,-2.2040209345,0$.

Al,0,-2.8678255377,-3.8295888446,0.

$\mathrm{Al}, 0,-1.9231397628,-1.3025811045,0$.

C,0,-1.0605263295,-3.0858470218,0.

C,0,1.7622517223,5.125868992,0.

Al,0,1.3085366784,3.8151631951,1.3992901778

Al,0,2.199434903,6.3886693683,-1.4885628372

Al,0,1.3085366784,3.8151631951,-1.3992901778

Al,0,2.199434903,6.3886693683,1.4885628372

Ca, $0,-2.4284138226,-7.0851886053,0$.

Ca,0,0.3647046889,1.0841011679,0.

\section{$\mathbf{H F}=-3370.7501356$}

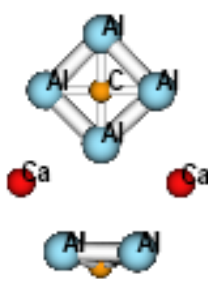

Al,0,-0.0087140439,5.0075302244,0.

$\mathrm{Al}, 0,-1.9753594806,2.9907089237,0$.

$\mathrm{Al}, 0,1.9656650519,2.9984225969,0$.

$\mathrm{Al}, 0,-0.0013606729,1.0885703238,0$.

C,0,-0.0050014226,3.0033097841,0.

C,0,0.0047562729,-3.2630621656,0.

Al,0,-1.3142013126,-2.7704706667,1.3930747186

Al,0,1.3233159889,-2.7663234676,-1.3923716622 Al,0,-1.3142013126,-2.7704706667,-1.3930747186 Al,0,1.3233159889,-2.7663234676,1.3923716622 
Сa,0,-2.861223348,-0.2948797562,0.

$\mathrm{Ca}, 0,2.8622977583,-0.2847629995,0$.

\section{$\mathbf{H F}=-\mathbf{3 3 7 0 . 7 8 8 4 2 5 8}$}

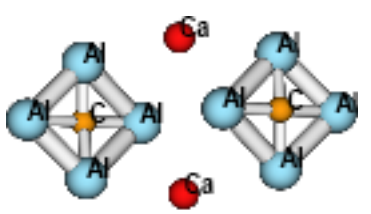

Al,0,-3.1614851754,1.9254304097,-0.1036945097 Al,0,-1.480699561,-0.0000000071,-1.2504951482 Al,0,-4.9288913831,-0.0000000406,0.660782204 Al,0,-3.1614851811,-1.9254303714,-0.1036945858 Al,0,3.1614883486,1.9254298061,0.1036854504 Al,0,3.1614883468,-1.92542981,0.1036854821 Al,0,1.4807151222,0.0000000071,1.2505241411 Al,0,4.9288589367,-0.0000000276,-0.6608355043 C, $0,-3.2340127495,0.0000000347,-0.4445000514$ C,0,3.2340128628,0.000000003,0.4444964486 Ca,0,0.0000033991,-2.5921769916,0.0000143754 Ca,0,0.000003422,2.5921770022,0.0000143112 HF $=-\mathbf{3 3 7 0 . 7 9 3 3 3 9 8}$

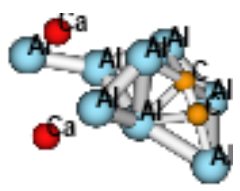

Al,0,-1.2862450631,-1.9081344972,-1.2441362851 Al,0,3.3641275433,-0.0145733512,-1.6363022573 Al,0,-3.4833138445,-0.475761464,-1.3666321548 Al,0,0.7869010987,-0.3890317663,-1.3876822626 Al,0,-1.0963674184,0.9351966202,0.0420055451 Al,0,-0.3284323878,-1.5551303875,1.2969324491 Al,0,0.8274548878,0.7013162038,2.0150965098 Al,0,-3.9951685721,1.6252911814,0.7338959107 C,0,-2.1253536583,-1.0781598243,0.4379833478 C,0,-2.9482297449,-0.0467547791,0.6733956062 Ca,0,1.9143744292,2.5439888022,-0.4503403752 Ca,0,2.9948790332,-1.5039765716,1.1223613434 HF $=-3370.7666943$

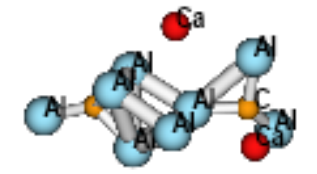

C,0,-2.8673918785,-0.55750222224,-0.4033748427 Al,0,-0.3210197835,-0.4579391636,2.1444439162 
Al,0,-1.4045442191,-1.7935834496,0.2023175113

Al,0,-1.625045332,0.446503414,-1.5933257106

Al,0,0.6644630429,-0.5277938801,-0.8737400901 Al,0,2.6153683001,1.666047807,-0.7045323797 Al,0,-2.5138612144,0.602736751,1.2104923828 Al,0,3.7698770855,-1.3236519332,-1.8888250814 Al,0,-4.6009115013,-1.2634955629,-0.9266830196 C,0,2.6072761278,-0.2506826493,-0.7827070021 Ca,0,-0.4342378603,2.8328709673,0.2007917246 Ca,0,2.7324604396,-0.8671510945,1.7344369349 HF $=-3370.7934362$

Al,0,0.6179943439,-1.6561306655,1.4211013743 $\mathrm{Al}, 0,-3.509397821,0.6363888621,0.1210509432$ Al,0,0.6600279454,-1.4104430128,-1.3983204449 Al,0,-1.9430177803,2.7250750725,0.1251293797 Al,0,2.9811874578,-2.0292557334,-0.0323271145 Al,0,-1.1304532651,0.3318760638,1.3929328161 Al,0,-0.9435511041,0.6109789295,-1.2689133262 Al,0,3.7568237224,0.5052923727,-0.0804389428 C, $0,0.4592540149,0.1738239379,0.189959463$ C,0,1.8171361485,-0.1004601262,0.1443392601 Ca,0,-2.3277645655,-2.5458640665,-0.0291244163 Ca,0,1.3948750985,2.6735230109,0.0482066941 HF $=-3370.7831268$

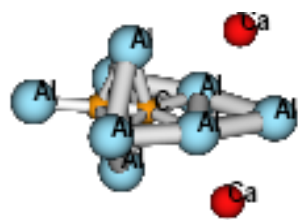

Ca,0,-3.2702158183,-1.1036096996,0. $\mathrm{Al}, 0,-0.8422822892,-3.0559866031,0$. Al,0,-0.2272858034,-0.8366751144,1.3958776823 Al,0,-1.6118092559,1.7931825459,0. Al,0,-0.2272858034,-0.8366751144,-1.3958776823 Al,0,0.4506221914,1.6246822565,-2.1112465918 Al,0,1.0899872837,3.9159704424,0. Al,0,0.4506221914,1.6246822565,2.1112465918 $\mathrm{Al}, 0,2.3093566318,0.7070862906,0$. C,0,0.5656547943,2.0306279102,0. C,0,0.1780214228,0.6342767968,0. 
Ca,0,2.2442478637,-2.6304668054,0.

\section{$\mathbf{H F}=-3370.7878581$}

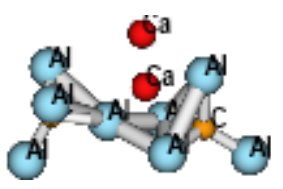

Al,0,-1.3116196145,-1.4594224884,1.1582093865 Al,0,-2.528932649,0.0295634479,-1.2554727353 Al,0,-4.3414989541,-0.0398644094,1.7004957687 Al,0,-1.3062789356,1.4298624355,1.2060544309 Al,0,2.9659809198,1.6309191459,-0.5340683561 Al,0,0.8252368123,-0.0126330506,0.6955836886 Al,0,2.9585458378,-1.6181286755,-0.5949213438 Al,0,3.6147918411,-0.0499081726,2.3717192 C, $0,-2.6429355329,-0.0037950699,0.7422670303$ C,0,2.8367193022,-0.0142322934,0.5545344 Ca,0,-0.319530199,-2.3039469695,-1.776636963 Ca,0,-0.3081513493,2.3676028272,-1.6983434917 HF=-3370.7783352

Ca,0,-3.5921919885,-0.8196761911,0. Al,0,-1.2159453355,-2.7911722855,0. Al,0,-0.1905184359,-0.743161245,1.4175698979 Al,0,-1.6391306213,1.8633641099,0. Al,0,-0.1905184359,-0.743161245,-1.4175698979 Al,0,0.3291799473,1.7979138843,-1.9935648323 Al,0,2.3461131251,3.372210309,0.

Al,0,0.3291799473,1.7979138843,1.9935648323 $\mathrm{Al}, 0,2.3188989346,0.2209512853,0$.

C,0,1.054997909,1.91141629,0. C,0,0.1886813033,0.7587707283,0. Ca,0,1.862369793,-3.0850380676,0.

\section{HF $=-3370.7861737$}

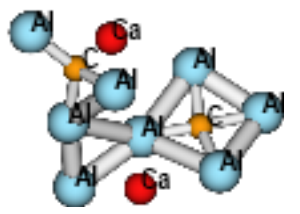

Al,0,-3.0175192134,1.0230261463,-0.9271735732 Al,0,1.6891974231,1.984672873,1.1801926553 Al,0,-2.1043945084,-1.9003984592,1.386256807 $\mathrm{Al}, 0,-4.3314470888,-0.5768307374,0.8598121984$ 
Al,0,0.9004869077,-0.4394155349,-3.0350800441

Al,0,-0.8030632396,-0.8278839923,-0.9560695017 Al,0,2.0626583042,-0.0364196973,-0.8315029034

Al,0,4.2796589927,-0.1300457789,1.7800205518

C,0,2.5232105062,0.2225105548,1.0648467125

C,0,-2.5119583985,-0.6654976051,-0.095197737

Ca,0,0.9959403157,-1.6550364054,1.7867104929

Ca,0,-0.1384413734,2.3750743879,-1.724301709

HF $=-3370.7861485$

G

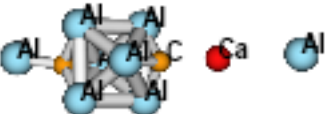

C,0,-1.6475685775,0.0988048405,-0.0920736071 Al,0,1.6465973263,-1.2245873073,0.516901796 Al,0,-0.5210616378,-1.0700897936,-1.2196480587 Al,0,-0.9322199199,1.8495066539,-0.6031558928 Al,0,1.4442355551,0.8496688332,-1.747030587 Al,0,1.2775173638,1.7661109809,1.149141952 Al,0,-0.6840959755,-0.1745480109,1.6116682529 Al,0,4.3113746212,0.515460429,0.1203879256 Al,0,-7.5405624902,-0.7918917837,-0.1722346523 C,0,2.387213054,0.6015414587,-0.0165434363 Ca,0,4.4923566858,-2.7961262159,1.1772381245 Ca,0,-4.0813670982,-0.2801696647,-0.1175713959 HF $=-3370.7426577$

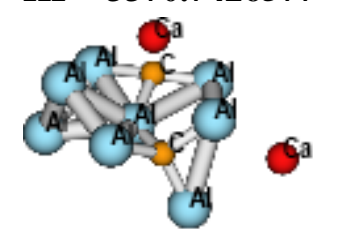

Al,0,-1.9962225449,0.3199392394,-2.063567047 Al,0,-3.0585792758,1.0514916212,0.187447742 Al,0,-0.3515947639,0.1195479031,0.4105579745 Al,0,-2.2953053095,-1.617873253,0.3451756588 Al,0,0.0906571167,1.967228444,-1.5194045358 Al,0,2.6399493602,-0.1504399033,-0.3947484187 Al,0,1.269613018,-2.3038079176,1.3323005826 Al,0,2.0806786472,2.2355274989,1.3879690109 C,0,1.2079835674,1.2212435002,-0.084643039 C,0,-0.3901894009,-1.8591978078,0.5213955073 Ca,0,4.0714491418,-0.547087898,2.4028490635 $\mathrm{Ca}, 0,0.4046790278,-1.8672272891,-2.0772459936$ HF $=-3370.769041$ 


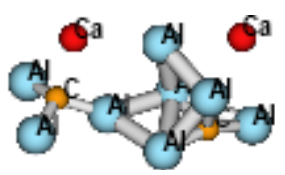

Al,0,-4.208510712,-1.3788999407,-0.099615151 Al,0,0.5885625933,1.0909502285,2.044877701 Al,0,-3.8251854557,1.7556604279,1.1137206296 Al,0,2.3370710853,1.4005297442,0.0998217179 Al,0,-1.3765367882,-0.1907191272,0.9110054157 Al,0,3.6295617946,-1.2208736912,1.772944903 Al,0,0.9081253529,-1.3515271028,0.5362959572 C,0,-3.1105273056,0.1967885614,0.1744554966 Al,0,0.6842363237,0.3196035713,-1.6707502868 C,0,2.1275642697,-0.0658345209,1.5390378337 Ca,0,3.4507979584,-1.2890605555,-1.4164117773 Ca,0,-2.3351697736,0.9737036719,-2.158031798

$$
\mathbf{H F}=-3370.778441
$$

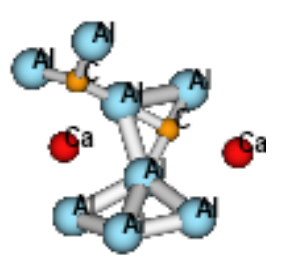

Al,0,3.2003092587,-0.9198994891,0.7008929977 Al,0,2.0959085965,-2.0212224564,-1.3581107008 Al,0,-3.1030970335,-2.6067573228,-1.2417877567 Al,0,-3.3389311322,-1.1545701357,1.7609316374 Al,0,3.0646447301,1.5976655379,1.1478245186 Al,0,-1.7674456223,2.7360075621,-1.4491179974 Al,0,-1.4847770967,0.0904301425,-0.5064029357 Al,0,1.1982594928,0.4466621739,-0.4743079379 C,0,-0.3766611973,1.6170967483,-0.7208918661 C,0,-2.3032039358,-1.4902922717,0.1507341199 Ca,0,0.8691173656,3.6413668401,0.2490222097 Ca,0,0.0226758987,-2.4888135912,0.8450759278 $\mathbf{H F}=-3370.7538835$

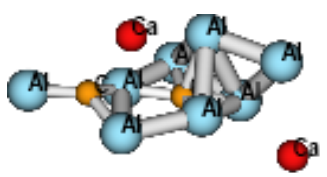

Al,0,-1.0250689355,-1.4874200567,0.6185758189 Al,0,-3.2694497899,-0.1138503271,0.3239033669 Al,0,-1.6914500195,-0.4302529316,-1.9542697101 Al,0,-0.4777509411,1.2325837664,0.0137416489 Al,0,0.2166151586,-2.2966789766,-1.8970686831 Al,0,4.8897107217,-0.4260262592,0.6914237854 Al,0,1.9534745267,-0.8453898035,-0.7002206843 
C,0,0.0628642169,-0.3438056786,-1.0308947759

Al,0,2.1557841023,1.789892177,0.5722540554

C,0,3.0147962731,0.0512415133,0.6452844207

Ca,0,1.2865710053,-0.6233902731,2.5133230961

Ca,0,-3.111548849,2.572900462,-1.1786325757

HF $=-3370.7751292$

Al,0,-1.4478978845,0.422835752,0.4188960025 Al,0,-1.2536396246,3.0088807854,-0.3849439491 Al,0,-0.5327828905,-2.2238465082,0.7435950658 Al,0,-3.736280566,-1.8051498306,1.5628365459 Al,0,0.8271554401,-0.3910976938,2.0702333523 Al,0,0.9675174221,1.7295487719,0.141694136 Al,0,-2.5302526838,-1.4673258895,-1.6559519036 Al,0,1.4353162793,-1.0539269319,-0.6981495463 C,0,2.0939194363,0.2191885406,0.663516028 C,0,-2.310023851,-1.3732366546,0.2710145179 Ca,0,4.5495117804,0.7251173682,1.0465125584 Ca, $0,-0.4086185259,0.7781500701,-2.7557080294$ HF $=-3370.7655514$

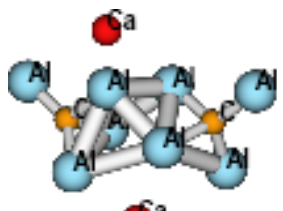

C,0,1.9185906084,1.1627843825,0.4881615126 $\mathrm{Al}, 0,0.6946261196,2.5386861535,1.2913902681$ Al,0,1.1699992208,-0.3735543573,1.5758751092 Al,0,3.7481372985,0.7752408278,0.065456338 Al,0,0.8516973009,0.6831008419,-1.1605079871 Ca,0,2.6438889352,-2.0516707696,-1.1011027995 Al,0,-3.1813063292,-0.289584406,-1.0595211704 Al,0,-0.3124799302,-1.6251238658,-0.285827163 Al,0,-1.3871467581,0.2760253789,1.2722088644 Al,0,-2.9655682745,-2.9187111665,1.0992892968 C,0,-2.1907801824,-1.3202461546,0.2639400795 Ca,0,-1.6639051841,2.7059576871,-0.9434639895 HF $=-3370.7908477$ 


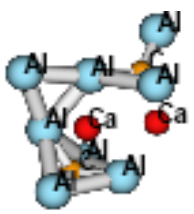

Al,0,3.4911887199,0.4686447099,0.5343017688 Al,0,-1.8893153313,-1.0286001717,1.9205155818 Al,0,1.0745663328,1.7270085458,1.3419339098 Al,0,0.8339380411,2.4516372598,-1.5383213965 Al,0,0.8719727343,-2.700469722,-1.9688639027 Al,0,1.6162060306,-0.2743428716,-1.4832443448 Al,0,-0.92878334477,-1.1954054894,-0.8428862864 Al,0,-4.175021216,-0.5815092242,-0.5756269729 C,0,-2.3894633976,-0.3753933136,0.1505810154 C,0,1.9695577496,1.3347787329,-0.4048423147 Ca,0,-1.8439913231,2.1223742425,0.1497783225 Ca,0,1.3883742391,-1.6737158421,1.6244246351 HF $=-\mathbf{3 3 7 0 . 7 8 6 8 1 4 8}$

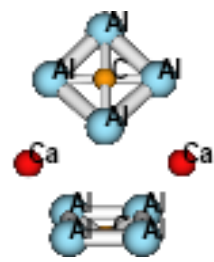

Al,0,-2.8879130677,1.943213053,-0.0320704614 Al,0,-1.1838218408,-0.0009486133,-1.0587510904 Al,0,-4.6817544198,-0.0102446162,0.746258 Al,0,-2.8810108473,-1.9539448501,-0.0331651887 Al,0,3.0644655984,-1.3217513962,-1.0258214874 Al,0,3.0576294579,1.3331897007,-1.0248364864 Al,0,2.2903156181,1.3214050398,1.6782107499 $\mathrm{Al}, 0,2.2985893002,-1.3162224205,1.6769291623$ C,0,-2.9359619925,-0.0057247935,-0.2644148061 C,0,3.103170022,0.0055867669,0.4465238966 Ca, $0,0.2700913357,2.8128647373,-0.3276621057$ Ca, $0,0.2800213861,-2.8093756624,-0.3293602$

\section{HF $=-3370.7899524$}

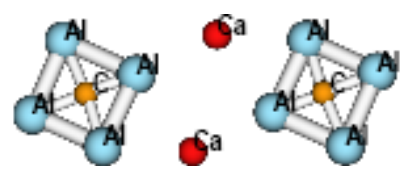

Al,0,0.7031889593,6.0750655254,0.3618303424

Al,0,-1.7644148584,4.977821134,-0.5222629411 Al,0,1.7573725023,3.5857449685,0.6829495193 Al,0,-0.646024532,2.41637105,-0.1799884906 C,0,-0.0096932975,4.1864108077,0.0764195346 
C,0,0.0095418851,-4.1864188336,-0.0759910502 Al,0,-1.7575469896,-3.5857573713,-0.6824566842 Al,0,0.6465459512,-2.4163428629,0.178490601 Al,0,-0.7040482188,-6.0751146156,-0.3593477988 Al,0,1.7641777274,-4.9778270713,0.5229548365 Ca, $0,-1.9468102835,-0.4174102315,-0.689894675$ Ca,0,1.9473428553,0.4174381472,0.6883560298 HF $=-3370.7754307$

Al,0,-1.9549208534,2.6221043537,-0.1520833312 Al,0,1.912029998,0.1224782727,-1.9019061337 Al,0,-0.1336802808,-1.6152397751,-0.6962606529 Al,0,4.1784197665,0.2413657728,-0.4048480902 Al,0,-2.3060604551,-1.0285249872,2.0659811709 Al,0,-0.5912701033,0.9272975409,-1.8226802368 Al,0,2.3328854445,-1.5917740239,0.2134678392 Al,0,0.36676953,-1.5296387503,2.0663377804 C,0,-1.192526529,0.8995153324,0.4167051178 C,0,-0.6742486205,-0.2564045419,0.8613722083 Ca,0,-3.238946396,-0.5974075709,-0.9144190803 Ca,0,1.3262664607,1.6082298714,0.9417904577 HF $=-3370.7823082$

Al,0,0.516668302,2.2671569709,-0.082465632 Al,0,2.9120878813,2.7217255422,0.8852789795 Al,0,2.5963516331,0.8011898542,-1.2645755976 Al,0,2.0071226533,0.3267186462,1.4554328682 Al,0,-1.8948279653,1.2017806366,-0.6858929798 Al,0,-2.9727292491,-1.7953120469,0.4281192267 Al,0,-0.5275386837,-1.7824798518,1.2168037242 Al,0,0.3319458334,-0.4342348358,-0.9053597218 C,0,-1.5217410773,-0.6998378346,-0.306587584 C,0,1.3051293022,-1.2888113713,0.7641428333 Ca,0,3.1349375492,-2.1732362127,-0.6611063122 Ca, $0,-5.0070664561,0.6109611407,-0.1786490928$ HF $=-3370.7530386$ 


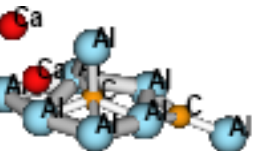

Al,0,-1.3721592933,-1.3062205935,-0.4320613943 Al,0,-2.7520999803,0.1611478352,-2.3052831511 Al,0,-0.346739238,1.0556036362,-1.3421946505 Al,0,-0.1457573744,1.0447086174,1.4742953505 Al,0,0.5968574385,-2.1691503603,1.1042391661 Al,0,5.4304226421,-0.0194193244,-1.1469486686 Al,0,2.3101953993,-0.7073825845,-0.0301574183 C,0,0.3201879948,-0.3033412973,0.0220101274 Al,0,2.2773681516,1.396099178,-1.8843743126 C,0,3.5320310864,0.2721522728,-1.0564316559 Ca,0,-1.9413899647,-1.3391649864,2.8840283372 Ca,0,-3.1130327942,1.702520531,0.3919134225 HF $=-3370.7713206$

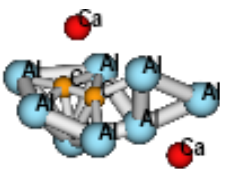

Al,0,-1.3516771847,-1.2360792825,0.9394983829 Al,0,-0.7790014188,-0.0112888831,-2.0625082985 Al,0,0.5679855158,-2.1483478548,-0.6458449856 Al,0,-2.9379336014,0.5637883723,2.0987297229 Al,0,1.1596272153,-0.7132862284,1.9206480976 Al,0,1.9074026053,-0.1924841865,-2.2721558621 Al,0,-0.7680870213,1.5005740356,0.7114043194 Al,0,3.4515291763,-1.3157715272,0.0649187419 C,0,0.4232802402,0.0829141543,-0.252672545 C,0,1.7307716631,-0.4037245112,-0.1669938832 Ca,0,-3.7160774982,0.3033943599,-0.882146471 Ca,0,2.257462491,2.1022308576,0.5174978224 $\mathrm{HF}=-\mathbf{3 3 7 0 . 7 8 8 5 1 3 1}$

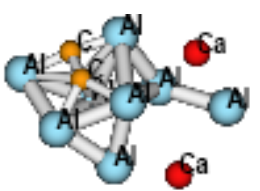

Al,0,2.0528170526,2.3949834368,0.1801845644 Al,0,-0.4866272051,2.4804309196,0.6605583274 Al,0,3.6689083218,1.1448354314,-1.7120975136 Al,0,-3.301547182,-1.6693944601,0.4987464808 Al,0,0.7280130215,0.6165607477,-1.6631377887 Al,0,0.5575894496,0.2038985626,1.2656127802 Al,0,-1.0225230076,-1.0909581694,-0.8157030801 Al,0,1.531506574,-1.9461920085,-0.5105904616 
C,0,2.1996746428,0.2921723649,0.0429972535

C,0,2.7065462317,-0.4563101178,-1.0181800534

Сa,0,-3.2937446959,1.4186992349,-0.21074551

Са,0,-0.6014106327,-2.756664808,1.8659776992

HF $=-3370.756558$ 
C,0,-2.8676370402,-0.5820470387,-0.3682865905 Al,0,-0.3285266825,-0.2916980584,2.1709929496 Al,0,-1.4090653551,-1.7713134397,0.3347691802 Al,0,-1.619869908,0.3263918868,-1.6272243041 Al,0,0.6656145163,-0.5937837631,-0.8278520177 Al,0,2.6209500622,1.6041761652,-0.8247358873 Al,0,-2.5174104171,0.698260197,1.1522145712 Al,0,3.7715455754,-1.4707233965,-1.7695352042 Al,0,-4.5979009706,-1.3317820267,-0.8393950805 C,0,2.6085147601,-0.3127410105,-0.7529574481 Ca,0,-0.4293366215,2.8405000227,-0.0184374954 Ca,0,2.7266043721,-0.7322565249,1.8048084724 $\mathrm{HF}=-3370.7934363$

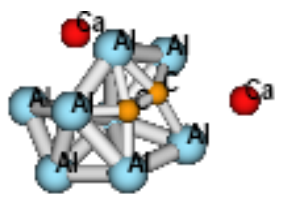

Al,0,1.9172437365,-1.7887652138,-0.1187698983 Al,0,-0.4744465418,-2.8737535384,0.2065707931 Al,0,-3.0476210774,0.7288054183,-1.1081797999 Al,0,-2.2884999836,-0.7748334689,1.1497456488 Al,0,-2.5812228688,-1.9008051125,-1.2092691621 Al,0,-0.578529579,1.0356276389,2.0088850166 Al,0,1.709214952,1.424478676,0.5155973531 C,0,-0.3384080708,-1.0136103318,1.3074461204 Al,0,-0.2604793644,-0.1747951625,-0.8117493788 C,0,0.8557815893,-0.3305003775,1.1452133539 Ca,0,-0.9943500804,3.1374933206,-0.4939159896 Ca,0,4.4819594971,0.0763663882,-0.653221725 HF $=-3370.767389$

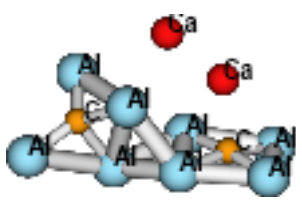

C,0,2.0948661187,-0.6264913582,-0.3868942311 Al,0,-1.4446093189,-1.3694894518,-1.2144524404 Al,0,0.6806532392,-1.9081328202,0.2319409568 Al,0,3.499326936,-1.7423735483,0.4655502344 Al,0,-1.2324468916,-0.4513065393,1.4579979164 
Al,0,-3.0098489064,1.2505503441,-0.1669192211

Al,0,0.6292488223,0.2709749403,-1.437273259

Al,0,-4.1353739526,-1.9946499405,-0.0700814919

$\mathrm{Al}, 0,3.4662617039,0.3030283302,-1.5490276061$

C,0,-2.7696101167,-0.6149356005,0.2009539369

Ca,0,1.6231830555,0.8415908387,1.804606251

Ca,0,-0.4153474167,3.1977463944,-0.2653519707

HF $=-3370.7892543$

$\mathrm{Al}, 0,-0.26849586,0.5484168301,-0.8840849733$

Al,0,-0.8013183989,-2.2692141639,-0.6351789662

Al,0,-1.5970056753,0.0382506096,1.6448621699

Al,0,1.6476006086,-1.5763234183,-1.0447731893

Al,0,1.7416746657,1.1392825564,1.3690541245

Al,0,0.6708720509,-1.3699061637,1.4651279044

Al,0,3.3872987524,-0.9505374209,1.0211471587

Al,0,-3.1991138304,1.9825306041,0.3866319819

C,0,0.0009015924,1.7549009928,0.8417989567

C,0,-1.2788240532,1.9475987776,0.6603159126

Ca,0,2.8780715252,1.3570249856,-1.6649594254

Ca,0,-3.5226777904,-0.8703995484,--0.9454860723

$\mathbf{H F}=-\mathbf{3 3 7 0 . 7 8 5 6 8 6 8}$

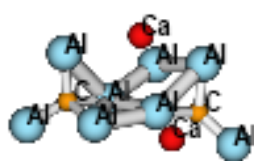

Al,0,-3.1010735348,1.4740106514,-0.5997609229

Al,0,0.6224554293,0.0313596015,1.166279584

Al,0,-1.9654091552,-0.208881633,2.0018012147

Al,0,-4.2197340195,-1.6738794364,-0.0807308024

Al,0,0.9918149506,0.1191452546,-2.28120453

Al,0,-1.0389360152,-0.6707121921,-0.6965842351

Al,0,2.532943003,1.4479263722,-0.3776862381

Al,0,3.8810392062,-1.0622048398,1.6089266736

C,0,2.4612530024,-0.2686303642,0.5870807629

C,0,-2.8143811975,-0.3188046085,0.0863812348

Ca,0,1.866240824,-2.4256063225,-0.8069661533

Ca,0,-0.2673172772,2.9549403584,0.1232510705

$\mathbf{H F}=-3370.7771783$ 
C,0,-2.722535163,0.2630625033,-1.1777790387

C,0,-2.7212399703,-1.0409125595,-0.6151017991

C,0,-2.7203120506,-0.9086947365,0.7988896914

C,0,-2.7206710876,0.4770714228,1.1099993066

C,0,-2.7219906699,1.2011742101,-0.1116271959

$\mathrm{H}, 0,-2.6858499943,0.5009403801,-2.2363569736$

H,0,-2.6849446535,-1.9741494346,-1.1684060457

H,0,-2.681446292,-1.7232506748,1.5154377785

$\mathrm{H}, 0,-2.681737547,0.9072761276,2.1059284479$

H,0,-2.6842972838,2.2813340048,-0.2127834082

Al,0,1.7163997458,-1.5877907998,-1.1854183202

Al,0,1.7145067439,-1.1835751387,1.588814503

Al,0,1.7130736882,1.5901470701,1.1841249421

Al,0,1.7148785417,1.1858486525,-1.5892266875

C,0,1.3922254241,0.0006688291,-0.0005437218

$\mathrm{Li}, 0,-0.8199154938,-0.002185204,-0.0015569802$

\section{$\mathrm{HF}=-\mathbf{1 2 0 8 . 9 3 7 4 6 8 6}$}

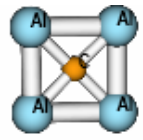

由)

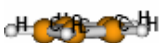

Al,0,1.4358744367,-3.7271588149,-0.0106923031 Al,0,1.3877596073,-0.9376059454,-0.0109040925 Al,0,-1.3728543798,-0.9462538832,0.0090392973 Al,0,-1.4045641136,-3.7353927567,0.0100561359 Li,0,-0.0052076083,1.7695781782,-0.000824277 C,0,1.1891918743,3.7367459154,0.0046610812 C,0,0.35819979,3.7315536781,-1.1457310533 C,0,0.3513747039,3.7290889254,1.1501218773 H,0,2.2739312925,3.6995395851,0.0078136657 C,0,-0.9942087315,3.723759096,-0.7108685081 H,0,0.6979812762,3.7052071471,-2.1770409039 C,0,-0.9984093645,3.7226447642,0.7073465874 $\mathrm{H}, 0,0.6850553604,3.7007487193,2.183376769$ H,0,-1.8708687171,3.6801529295,-1.3499059479 H,0,-1.8787708576,3.6777244679,1.3411528861 C,0,0.0117671131,-2.3552522428,-0.0005985057 $H F=-1208.9398322$ 


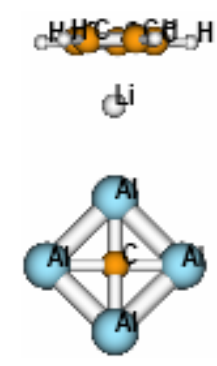

C,0,-4.1571714879,0.69278992,-0.5102296677

C,0,-4.1361227699,-0.7224515868,-0.6287019767

C,0,-4.0316598958,1.0197048902,0.8651119162

H,0,-4.2239575124,1.4012679025,-1.3301687986

C,0,-3.9981238659,-1.2699102952,0.6734883061

H,0,-4.1859128005,-1.2865395003,-1.5550982055

C,0,-3.9325182848,-0.1933141133,1.596802876

H,0,-3.9826577885,2.0215868721,1.280168699

H,0,-3.9181102388,-2.3248316872,0.9166056222

H,0,-3.801421388,-0.2815519944,2.6710876199

Al,0,4.5093542575,0.1080446252,-0.4490450448

Al,0,2.5044667,2.0475069131,-0.2780523685

Al,0,0.598908963,0.0112413123,-0.0551561128

Al,0,2.6079348341,-1.9278167259,-0.2262850213

Li,0,-2.1397707663,-0.0510926713,0.2159104994

C,0,2.532718007,0.0592906519,-0.2496923405

\section{HF $=-\mathbf{1 2 0 8 . 9 3 9 5 6 2 5}$}

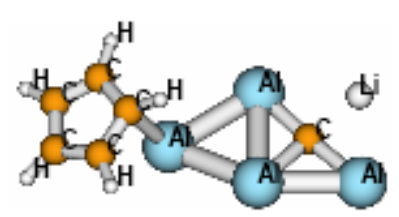

C,0,-2.9909985875,-0.7375582131,0.6201980062

C, $0,-2.2133503846,0.4468036336,0.86748776$

C,0,-4.0536706285,-0.3904983887,-0.2274574744

H,0,-2.7720326656,-1.7243430832,1.0142511025

C,0,-2.8618782981,1.5248892576,0.1740737308

H,0,-1.4816925167,0.5600245245,1.6622326227

C,0,-3.9740623696,1.0026522426,-0.501462575

H,0,-4.7723885597,-1.081731227,-0.6596011867

H,0,-2.5219134567, 2.5552440905,0.1605659992

H,0,-4.6207818299,1.5526620224,-1.1801111037

Al,0,1.390087225,-1.3040059689,0.2652262736

Al,0,4.1625471225,-1.325966874,0.5927600728

Al,0,1.6071278203,1.3870533452,-0.4574838089

Al,0,-0.8076843187,-0.0590916431,-0.8752992898

Li,0,4.3310721621,1.3136349546,-0.2024611763

C,0,2.8603903524,0.0076087414,0.0308945315 


\section{$H F=-1208.8640558$}

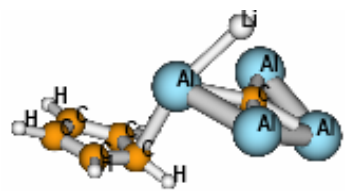

C,0,-3.5028584449,-0.6242828385,-0.4687020717

C,0,-2.326435489,-0.895326673,0.2338816216

C,0,-1.7709815394,0.3599062925,0.6819342759

C,0,-2.7053673304,1.3852093251,0.2924403984

C,0,-3.7368924708,0.7845355335,-0.429922341

H,0,-4.1049205157,-1.3528528122,-1.0063737481

H,0,-1.8809481109,-1.8717856658,0.3938030702

H,0,-1.0557012054,0.4542227422,1.4952130231

$\mathrm{H}, 0,-2.5795937138,2.4474849948,0.4771083748$

H,0,-4.5483343525,1.3047151655,-0.9349031844

Al,0,1.9193662264,1.7481739478,0.4911303189

Al,0,-0.1839009993,0.4464599007,-0.9813927756

Al,0,3.0167976257,-0.6025574793,1.3326978501

Al,0,1.6340271694,-2.0890848011,-0.4959808364

C,0,1.6298893314,-0.1577936534,-0.0572848012

Li,0,1.8745344228,0.1219457579,-2.1476130895

\section{$\mathrm{HF}=\mathbf{- 1 2 0 8 . 8 6 9 8 4 6 7}$}

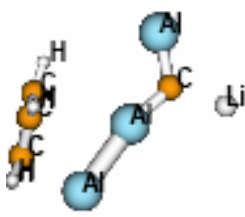

C,0,-3.0111986536,0.5641009426,-1.1445642119

C,0,-3.4939757699,-0.7005761409,-0.721379189

C,0,-2.7246625834,1.3403286739,-0.0010436778

H,0,-2.853251928,0.8668261149,-2.175294663

C,0,-3.4931032113,-0.7022247395,0.7155879732

H,0,-3.943180864,-1.4584318674,-1.3567993315

C,0,-3.009892492,0.5616403744,1.1410323001

H,0,-2.2555772457,2.3190510735,-0.0002095185

H,0,-3.9423053597,-1.4611086814,1.3497904395

$\mathrm{H}, 0,-2.8508640971,0.8621275307,2.1722433781$

Al,0,-1.6399803037,-2.1753409222,-0.0039117039

Al,0,6.3985719409,-1.2137747837,-0.0091612887

Al,0,0.2996785978,-0.2137045995,-0.0005815239

$\mathrm{Al}, 0,1.7531181014,2.675295823,0.0174010987$

Li,0,3.5934753876,-0.1037339184,-0.0016689181

C,0,1.8189502031,0.8101568655,0.0047952856

HF $=-\mathbf{1 2 0 8 . 7 9 5 5 8 8 6}$ 


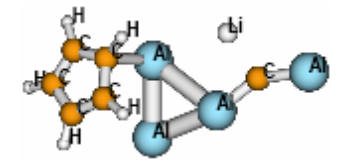

C,0,3.2777316496,0.6184191307,0.8605422357

C,0,2.1133591406,-0.0331198468,1.2963880448

C,0,3.9106196381,-0.2026273892,-0.1143943287

H,0,3.5976842979,1.6122600907,1.1599606173

C,0,1.9946820105,-1.2750320907,0.5719481691

H,0,1.4322493788,0.3083276646,2.0698923828

C,0,3.1522450885,-1.3675760338,-0.2766702307 H,0,4.7979093799,0.0626129837,-0.6841361712 H,0,1.4085068547,-2.1260018356,0.9094311818 H,0,3.3689449596,-2.1897683356,-0.9526000913 Al,0,0.4511479645,-0.6641662977,-1.0509324729 Al,0,-5.0529451925,-0.4950141083,0.3832026856 Al,0,0.5735478308,1.9868600859,-0.5772733991 Al,0,-1.7567612527,0.8515847215,0.22548187 Li,0,-2.1933752854,-1.5624522664,-1.0731539559 C,0,-3.2519759551,-0.2084822681,-0.0093653801 HF $=-\mathbf{1 2 0 8 . 8 3 1 3 9 2 5}$

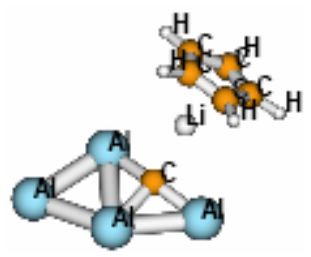

Al,0,2.4264852587,0.9244663,0.9433433288

$\mathrm{Al}, 0,0.2451460302,2.5599375243,0.1621667645$ Al,0,1.581457033,-0.8146477227,-0.9648211887 Al,0,4.0320647297,-1.2221293486,0.3438125253 C,0,0.7670381204,0.7303395377,-0.1382582157 Li,0,-1.2421349606,-0.0493077487,-0.2196768752 C,0,-3.480798026,0.4615558384,-0.3844707028 C,0,-3.187543299,-0.5498501646,-1.3369198089 C,0,-3.2577384023,-0.0777494794,0.9097350772 H,0,-3.7804616357,1.4811046935,-0.6060758303 C,0,-2.7833742827,-1.713746347,-0.6314684219 H,0,-3.2281210865,-0.4412467028,-2.4168454933 C,0,-2.8270166221,-1.4215360758,0.7567705903 H,0,-3.3581253125,0.4583851952,1.8486943488 H,0,-2.4548224015,-2.6475337752,-1.0766995044 H,0,-2.5424592829,-2.0960138097,1.5591074065 HF $=-\mathbf{1 2 0 8 . 9 0 7 1 8 3 5}$ 

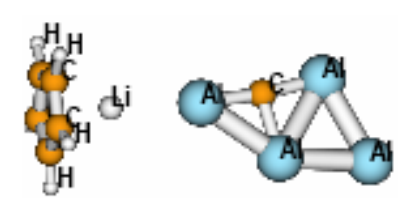

Al,0,-3.2250106036,-2.3006999861,-0.817194015 Al,0,-0.4616915201,0.1487149263,-0.0168961149 Al,0,-0.5523250159,-2.7133495223,-0.3314524735 Al,0,-2.10580859,-4.9077300139,-0.8556610154 C,0,-1.7637145028,-1.1374304088,-0.4126927134 Li,0,1.3288587428,2.2075664788,0.4693553096 C,0,3.2175183823,3.2660604008,-0.1514294544 C,0,2.1488914541,4.1993741154,-0.2107245983 C,0,3.3594710667,2.846915602,1.1973856536 H,0,3.8051134808,2.9140453079,-0.9935934685 C,0,1.6307357501,4.3571512391,1.1016010068 H,0,1.7780834347,4.6889862956,-1.1059599778 C,0,2.3788922348,3.5209648623,1.9719864261 H,0,4.0732630779,2.1171729318,1.5664482894 H,0,0.7943294495,4.9883479924,1.385540441 H,0,2.2147325026,3.4003729189,3.0383879098 HF $=-\mathbf{1 2 0 8 . 8 9 9 4 7 3 2}$

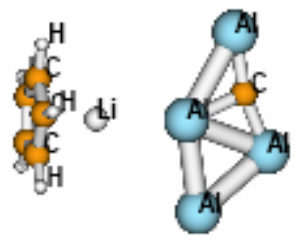

Al,0,1.0844299669,0.1702936376,0.102854878 $\mathrm{Al}, 0,2.211218408,2.9431134469,0.7388172133$ Al,0,3.709925965,-0.4676156548,0.1038907421 Al,0,1.8496104981,-2.4796662177,-0.4047161296 C,0,2.7566934398,1.1652931276,0.3960184479 Li,0,-1.7146351971,-0.1012331755,-0.1079338765 C,0,-3.7089234406,-0.3299236316,0.9417997485 C,0,-3.7466107565,0.8659851327,0.1772052397 C,0,-3.5470493664,-1.4196591881,0.0456298146 H,0,-3.7638427425,-0.3966222174,2.0242146987 C,0,-3.6076504244,0.515329792,-1.1913132558 $\mathrm{H}, 0,-3.8281398461,1.8738417675,0.572204011$ C,0,-3.4844385323,-0.8973385616,-1.2725942331 H,0,-3.4506720966,-2.4651038372,0.3210583326 H,0,-3.5696129541,1.2087954249,-2.0259414377 H,0,-3.3333551802,-1.4749593927,-2.1792156971 HF $=-\mathbf{1 2 0 8 . 8 9 7 3 6 9 1}$ 


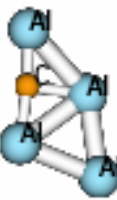

Al,0,-2.2402265918,-3.3591948382,-0.1684726651 Al,0,-0.4454979848,-0.0429717104,0.0878405568 Al,0,0.3930683243,-2.7062809571,-0.5985261869 Al,0,-0.3402117316,-5.321152518,-0.9428978482 C,0,-1.2544516377,-1.7251400123,-0.0641733881 Li,0,0.5161856428,2.5396361159,0.3786944162 C,0,2.2650862803,3.9567391386,0.3013218821 C,0,1.2823121411,4.4131217247,-0.6158963812 C,0,1.7065863598,4.0105303541,1.6054443643 H,0,3.2585383223,3.6008695228,0.0470488675 C,0,0.1163207323,4.7498093528,0.1215140774 H,0,1.3945592661,4.4710868928,-1.6941712795 C,0,0.3785145827,4.5010209526,1.4943543706 H,0,2.2002553261,3.7071157976,2.5235369062 $\mathrm{H}, 0,-0.8193638232,5.1112484467,-0.2937882429$ $\mathrm{H}, 0,-0.3214729802,4.6390822372,2.3126308131$

\section{HF $=-\mathbf{1 2 0 8 . 8 9 9 4 7 4 7}$}

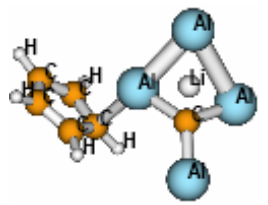

C,0,-2.7790077242,-0.7496666767,-0.7450649158 C,0,-2.0653473018,-0.0617548668,0.287489123 C,0,-3.1463251245,0.1836924754,-1.7227734934 H,0,-2.9352986409,-1.8226575747,-0.7894486653 C,0,-2.0528491213,1.3371985495,-0.0712124339 H,0,-1.8446092648,-0.4536647394,1.2761618797 C,0,-2.698694483,1.4674236989,-1.3127419614 H,0,-3.6191737578,-0.0462042052,-2.6741110061 $\mathrm{H}, 0,-1.6550022263,2.140120626,0.5408327083$ H,0,-2.7871354544,2.3856473844,-1.8862896308 Al,0,2.2378623963,-1.5448602347,1.1964093794 Al,0,2.0529943285,-1.2299233291,-1.5852148274 Al,0,1.2431226394,1.2002862134,2.5117625767 Al,0,0.0579038334,0.0192417219,-0.527176717 Li,0,2.678658689,1.187102001,-0.4137480339 


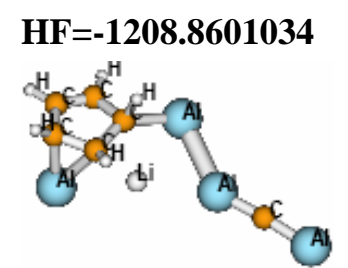

C,0,1.1535250991,3.5521382134,-1.224401483

C,0,1.6364134994,2.4967509887,-2.1286374832

C,0,0.1756576734,3.1093743715,-0.38588962

H,0,1.5701887432,4.5602056183,-1.1998905263 C,0,0.9323315106,1.2532502941,-1.6185109537

H,0,1.7592883384,2.7174651442,-3.1939639024 C,0,-0.2377713202,1.6844585948,-0.6822214421 $\mathrm{H}, 0,-0.3043418249,3.7237912821,0.3749497144$ H,0,0.698072379,0.47982816,-2.35951546 H,0,-1.2040612238,1.7206441687,-1.2648788752 Al,0,-2.0541158692,-5.2988554486,1.8665146894 Al,0,-1.4906149,0.8297778695,0.8473771458 Al,0,-0.8645683977,-1.9302035556,0.9755539958 Al,0,3.0011039916,1.4604233066,-0.9709544168 Li, $0,0.900576707,-0.0323347722,-0.025749643$ C,0,-1.4792130047,-3.5792688442,1.4363542473 HF $=-\mathbf{1 2 0 8 . 7 7 4 3 8 9 1}$

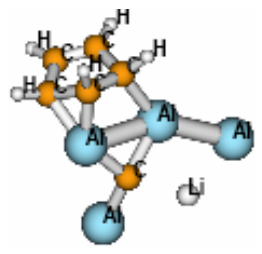

C,0,1.4610283542,2.6575039592,0.1687920879 C,0,1.8856690513,1.713581338,-0.9007903865 C, $0,0.1108128579,2.6729814744,0.2842954633$ H,0,2.147226977,3.202562367,0.8186638229 C,0,0.572096765,1.4826038169,-1.7775342665 H,0,2.7852725695,1.9731188024,-1.4719751521 C,0,-0.5208608324,1.6696080922,-0.6578745537 $\mathrm{H}, 0,-0.4506375033,3.2263844895,1.0364159664$ $\mathrm{H}, 0,0.4449559842,2.1458984319,-2.6470593589$ H,0,-1.4821090254,1.9974423944,-1.0800660221 Al,0,-3.1638335723,-1.3272753494,1.6846703423 Al,0,-1.1222181155,-0.102452405,0.4107946857 Al,0,1.4614734941,-2.7712454202,0.6515949138 Al,0,1.1697662133,-0.1682949489,-1.0115460454 Li,0,-1.4823907818,-2.5846505583,-0.5055625763 C,0,0.2437569856,-1.5281068816,-0.0670503745 


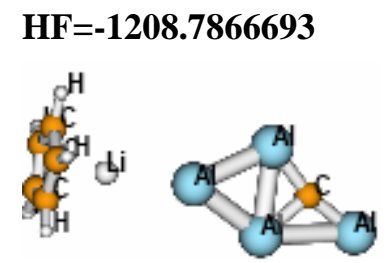

C,0,0.1699457536,4.3227303529,-1.805279996 C,0,0.7242785175,3.6171141561,-2.9050205952 C,0,-1.2389347236,4.1486593177,-1.8375864091 H,0,0.7280306172,4.8732084024,-1.0542038641 C,0,-0.3418348886,3.0068707397,-3.6171557842 H,0,1.7803985942,3.5334936257,-3.1420985881 C,0,-1.5551598152,3.3357680986,-2.9575378959 $\mathrm{H}, 0,-1.9474502937,4.544042652,-1.1161683673$ H,0,-0.2440614849,2.3771463652,-4.496198735 H,0,-2.5473609006,2.998825129,-3.2413392997 Al,0,-1.2879016578,-2.4296079741,1.2238000346 Al,0,-0.0429706387,-0.217090481,0.1155841713 Al,0,1.3865695006,-1.8669390756,1.696600376 Al,0,1.164381544,-4.3826654514,3.3430130216 Li,0,-0.2799564078,2.1095170005,-1.5280571083 C,0,0.1099199839,-3.2650307333,2.2404492359

\section{$\mathrm{HF}=\mathbf{- 1 2 0 8 . 9 0 1 5 3 7 7}$}

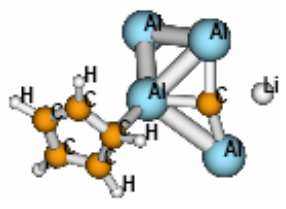

C,0,1.7346495475,0.6447860901,0.8925085562 C,0,2.5013033061,-0.5551542434,1.0835051611 C,0,3.3484055179,-0.713048683,-0.0176540748 C,0,3.1634180787,0.3936301519,-0.8935983077 C,0,2.1999099601,1.243432384,-0.3373665823 H,0,1.2004581158,1.1748205522,1.6760150668 H,0,2.3909507377,-1.2437354788,1.9153831657 H,0,3.9893813524,-1.5700950021,-0.2091564601 $\mathrm{H}, 0,3.6426024165,0.5245067647,-1.860171188$ H,0,1.8539446814,2.1868719122,-0.7473940564 Al,0,-0.0960284712,-0.1512373726,-0.1725052895 Al,0,-1.1371152987,-2.3038095726,-1.2780441855 Al,0,-1.8029933434,2.3806719373,-0.4984647872 Al,0,-2.4221929331,-1.0972556764,0.9039042804 Li,0,-3.0799289825,1.2080722614,1.9046687703 C,0,-1.7608963689,0.7421231941,0.4555630683

\section{HF $=-\mathbf{1 2 0 8 . 8 5 1 8 5 8 5}$}




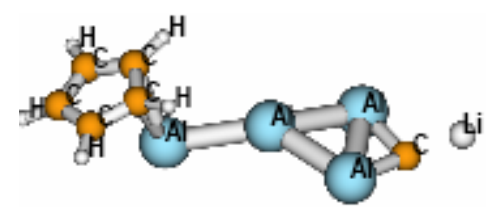

C,0,-2.771802644,0.6559360762,-0.9015432684

C,0,-2.8562996351,-0.7737953113,-0.8512412307

C,0,-4.0402772645,-1.1071290896,-0.1416634309

C,0,-4.6868709227,0.0899804396,0.2347778864

C,0,-3.9052090135,1.1741621243,-0.2198355036

$\mathrm{H}, 0,-2.0313023844,1.2274631965,-1.4507981847$

$\mathrm{H}, 0,-2.1887294401,-1.4648354591,-1.3549365447$

H,0,-4.3685588462,-2.1140834017,0.0965099962

H,0,-5.5707250623,0.1638367558,0.8610189342

H,0,-4.1125272091,2.2267363961,-0.0543776824

Al,0,-1.6143692914,-0.0413727616,1.0913509264

Al,0,1.0560156354,-0.176520309,0.0485320325

$\mathrm{Al}, 0,3.227044728,1.4721174631,-0.0846832813$

Al,0,3.5382470999,-1.2280103464,0.0096101559

Li,0,6.2662826879,-0.8326086277,-0.0672538423

C,0,4.7242592536,0.3138333936,-0.0768582574

HF $=-\mathbf{1 2 0 8 . 8 1 3 2 7 1 3}$

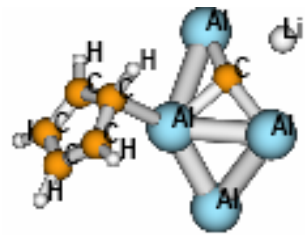

C,0,3.346100264,0.7114403022,0.2359745263

C,0,2.4504310944,0.4318596633,1.2728283338

C, 0,1.690275045,-0.7311335932,0.9089741756

C,0,2.2082741849,-1.1854638205,-0.3610230251

C,0,3.1977059342,-0.283319581,-0.7710921499

H,0,3.9969417552,1.5802244951,0.1748288943

H,0,2.3037950386,1.0196830005,2.1735842209

H,0,1.1177057801,-1.3438572997,1.5996089538

H,0,1.8807621323,-2.0730074026,-0.8929019529

$\mathrm{H}, 0,3.7193587211,-0.3041488968,-1.7241858124$

Al,0,-1.0974147019,2.4593456427,-1.0006662122

Al,0,-0.0890112396,0.1784561872,-0.1554052559

$\mathrm{Al}, 0,-2.481666873,0.9454218242,0.9163254423$

Al,0,-1.7352840027,-2.294714979,-0.925386444

Li,0,-3.1674862438,-1.4834006792,1.5344400304

C,0,-1.7714875345,-0.8066004098,0.2497494248

HF $=-\mathbf{1 2 0 8 . 8 5 1 8 5 9 1}$ 


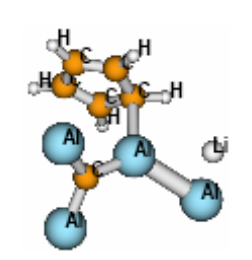

C,0,2.77049992665,-0.1534348195,0.9590437374

C,0,1.9079538457,-1.2483292694,0.9627958837

C,0,1.4144418719,-1.4423804173,-0.3839088558

C,0,2.1002981472,-0.4691684208,-1.2085624546

C,0,2.8895331642,0.3253523403,-0.3825899291

H,0,3.2275190122,0.2996090522,1.8344247054

H,0,1.6190542403,-1.8383551354,1.827128817

H,0,1.0772861507,-2.4085571269,-0.7566389884

H,0,1.9677587463,-0.3528018703,-2.2800035803

H,0,3.4422392102,1.2100073452,-0.68861755

Al,0,-0.3590411304,1.6049239619,2.2233579336

Al,0,-0.6403069537,-0.5183401911,-0.2220688601

Al,0,-1.6345464574,2.512035085,-0.7498091148

Al,0,-2.3852776388,-1.9408099059,-1.6658674119

Li,0,-2.3838592722,-2.6951431427,1.0236318129

C,0,-0.9048998287,1.2586290558,0.4498629602

HF $=-\mathbf{1 2 0 8 . 8 3 8 4 4 2 2}$ 


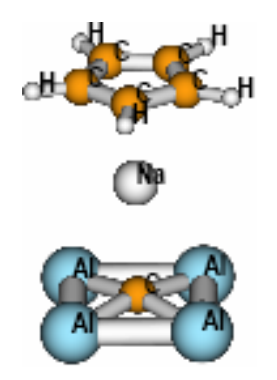

C,0,3.1783154097,0.075979947,-1.2094938662

C,0,3.1755913852,1.1754315953,-0.3103650102 C,0,3.1802064743,0.6601105581,1.0133118539 C,0,3.1886518578,-0.7579371053,0.9323074764 C,0,3.1869454212,-1.1188633772,-0.4415278883 H,0,3.1657300754,0.1381252673,-2.2944652506 H,0,3.1603718496,2.2264833817,-0.586682149 H,0,3.1684494575,1.2479083235,1.9274106326 H,0,3.1840765894,-1.4456631647,1.7738499605 H,0,3.1804765002,-2.1315442785,-0.8359204518 Al,0,-2.0960524747,1.6233411167,1.1424480491 Al,0,-2.0970530704,1.1373079273,-1.6251013971 Al,0,-2.0910521537,-1.6311599993,-1.1401568166 Al,0,-2.0894213428,-1.145258749,1.6294708454 $\mathrm{Na}, 0,0.7702578461,-0.001259884,0.0012210711$ C,0,-1.8222794213,-0.0041290593,0.0017318721

\section{$\mathrm{HF}=\mathbf{- 1 3 6 3 . 6 9 4 8 7 5}$}

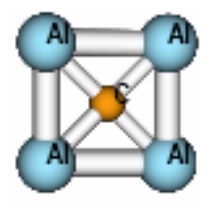

(1)

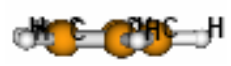

Al,0,1.4300090958,-4.1261673014,-0.0124630493 Al,0,1.4104074439,-1.3488921907,-0.0106200117 Al,0,-1.3971781801,-1.3624518071,0.0103701147 Al,0,-1.3896339218,-4.1399526591,0.0085806252 $\mathrm{Na}, 0,-0.0051943865,1.5852746168,0.0009643738$ C,0,1.1873939492,4.0543040366,0.0050165202 C,0,0.3562892739,4.0507117758,-1.1457426146 C,0,0.3493772486,4.0509133989,1.1507856074 H,0,2.2741663624,4.0468089616,0.0082704067 C,0,-0.9958390746,4.0455084511,-0.7110158888 H,0,0.6960619508,4.0489129476,-2.178534846 C,0,-1.0001155053,4.0457790434,0.7079761727 
$\mathrm{H}, 0,0.6829207103,4.0494127382,2.185616332$

H,0,-1.8737530277,4.0333310518,-1.3518265965

H,0,-1.8818406215,4.0336844204,1.3435201034

C,0,0.0133483059,-2.7377399468,-0.0010086862

$\mathrm{HF}=-\mathbf{1 3 6 3 . 7 0 3 3 0 1}$

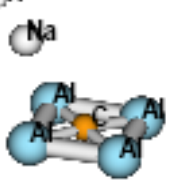

$\mathrm{Na}, 0,-1.104660701,-0.0252495015,-0.3580376965$ C,0,-3.3965703836,-1.1999821041,0.454158826 C,0,-3.0717592513,-0.2548569307,1.4632294909 C,0,-3.7524504287,-0.4839518433,-0.7195176213 H,0,-3.3695459658,-2.2813939385,0.5593518541 C,0,-3.2268388683,1.0452124213,0.9129649823 $\mathrm{H}, 0,-2.7546381301,-0.4858428367,2.4767695567$ C,0,-3.647825345,0.9035026691,-0.4359450649 H,0,-4.0441131058,-0.9219264924,-1.6706823293 H,0,-3.0477192155,1.983542504,1.43123991 H,0,-3.84389256,1.7148979315,-1.1320263476 Al,0,1.5843333223,-1.465017144,-1.2955472458 Al,0,1.5701669803,1.3972053802,-1.3770695461 Al,0,2.9871979915,1.4401618333,1.0198328093 Al,0,3.0018906162,-1.3581199631,1.0988531379 C,0,2.1528644192,0.00398844485,-0.0957467798 HF $=-\mathbf{1 3 6 3 . 6 8 9 2 3 2 8}$

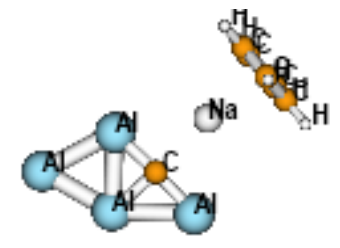

Al,0,-1.6904643587,-1.2811011564,-0.0013761443 $\mathrm{Al}, 0,-0.8978765524,2.499746437,0.000007965$ Al,0,-3.2109589905,0.9423487571,0.0005662014 Al,0,-4.4912413112,-1.4688560735,0.001030455 C,0,-1.2445324112,0.6015293809,-0.0010040191 $\mathrm{Na}, 0,1.2228057915,-0.0286245433,-0.0052971888$ C,0,3.8016513773,0.8231270141,-0.2397141463 C,0,3.7238538679,0.2400240502,1.0522353163 C,0,3.6731593348,-0.2168793671,-1.1980528363 H,0,3.9206434244,1.8813476933,-0.456098846 C,0,3.5478056251,-1.1604624172,0.8923294109 
H,0,3.7822817711,0.7740157666,1.9974684527

C,0,3.5164849185,-1.4426599829,-0.4980727271

H,0,3.6883111867,-0.0952465155,-2.2785080757

H,0,3.4440028477,-1.8881364736,1.6932753555

H,0,3.3803965543,-2.4229760972,-0.9471640028

$H F=-1363.6656651$

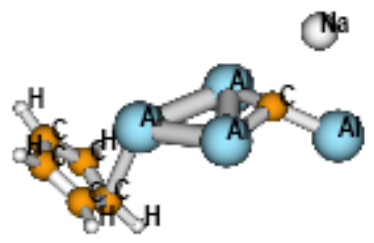

C,0,-2.8118367621,0.4088078484,1.1951521159

C,0,-3.2254122403,1.2612130423,0.1275864291

C,0,-3.8869496396,0.4640538932,-0.8362970368

C,0,-3.8922269988,-0.8776400048,-0.3740755918

C,0,-3.2351978117,-0.916631961,0.8783019612

H,0,-2.3105835144,0.7200758265,2.1045487722

H,0,-3.0298244307,2.3246518506,0.0553945624

H,0,-4.262806574,0.8081445005,-1.7932985866

H,0,-4.2726422829,-1.7353771928,-0.9173427463

H,0,-3.046144439,-1.8004233371,1.476192486

Al,0,-1.261876683,-0.1656647418,-0.4741982816

Al,0,1.0839777925,1.3854612359,-0.5839639853

Al,0,1.079065664,-1.4514370604,0.4222040901

Al,0,3.6258398979,0.4917481218,1.38149325

Na,0,4.237404622,-0.5313670458,-1.542325079

C,0,2.2948673976,0.0179584541,0.0673530275

$H F=-1363.6297665$

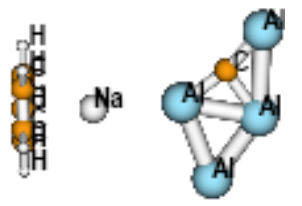

Al,0,4.0944096551,-2.6174763127,0.1579865553

$\mathrm{Al}, 0,1.2712407871,-0.0960638312,-0.0002316572$

$\mathrm{Al}, 0,3.9092355867,0.3953955287,-0.0586912804$

Al,0,2.2931198345,2.5775459455,-0.2008280709

C,0,2.8090020146,-1.2378715075,0.0690622514

$\mathrm{Na}, 0,-1.788999835,0.0887501089,0.0108794307$

C,0,-4.2174250326,0.6378132544,-1.0528767642

C,0,-4.2269569967,-0.7651955458,-0.8324383358

C,0,-4.2058989239,1.2809785081,0.2129905942

H,0,-4.2219193967,1.1331773548,-2.02041204

C,0,-4.2209485676,-0.9891378662,0.5693863296

H,0,-4.2370300857,-1.5330305792,-1.6017754276 


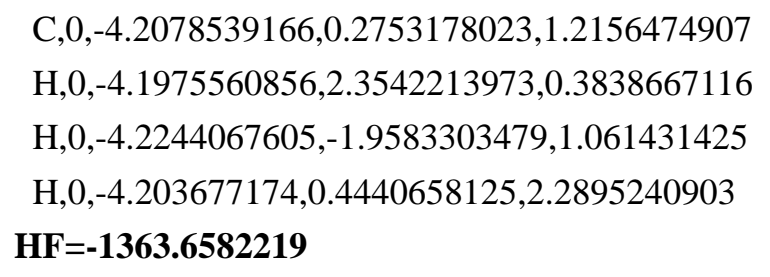

C,0,4.4646713313,-0.9275667778,0.6278829337 C,0,3.2164161469,-1.1768031626,1.2043820434 C,0,2.2804670676,-1.4609831457,0.143079703 C,0,3.0351350767,-1.4459772835,-1.0873874955 C,0,4.3529639713,-1.0930712378,-0.7845029865 H,0,5.3559514667,-0.6038134875,1.1582185852 $\mathrm{H}, 0,2.9688994375,-1.1245041189,2.2589551741$ H,0,1.3190245028,-1.9518501105,0.2772117497 H,0,2.6264817137,-1.6347093859,-2.0740823785 H,0,5.1449584996,-0.9158098868,-1.5068382124 $\mathrm{Al}, 0,0.7310710084,2.9629898954,-0.2706114208$ Al,0,1.6291402345,0.6671390295,-0.0634846269 Al,0,-1.0315680239,1.1858139965,0.0843481547 Al,0,-3.3463854391,0.0119433936,0.3230498784 $\mathrm{Na}, 0,-5.9918103723,-1.4346425998,-0.7552918782$ C,0,-4.8954457042,-0.6873928103,1.1035157905 $H F=-1363.5124473$

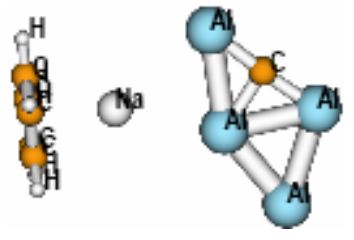

C,0,3.8906593633,-0.8378228357,1.1229692172^M $\mathrm{C}, 0,3.4485517731,-2.0362027373,0.5017929099 \wedge \mathrm{M}$ $\mathrm{C}, 0,4.1936577976,0.1003544946,0.1013876851^{\wedge} \mathrm{M}$ $\mathrm{H}, 0,3.9784705063,-0.6670757226,2.1931121892 \wedge \mathrm{M}$ C,0,3.4780295808,-1.8385435885,-0.9030637938^M $\mathrm{H}, 0,3.1333781665,-2.9426517922,1.0121431711^{\wedge} \mathrm{M}$ C,0,3.9383777252,-0.5178972892,-1.1508920072^M $\mathrm{H}, 0,4.5447669356,1.1176950237,0.2518880348 \wedge \mathrm{M}$ H,0,3.1894032419,-2.5672484671,-1.6561438434^M $\mathrm{H}, 0,4.0691296969,-0.0591184841,-2.1278745946 \wedge \mathrm{M}$ Al,0,-4.127677826,1.1505843891,0.0760939781^M $\mathrm{Al}, 0,-0.6345754933,2.6088499061,0.3505915609 \wedge \mathrm{M}$ 


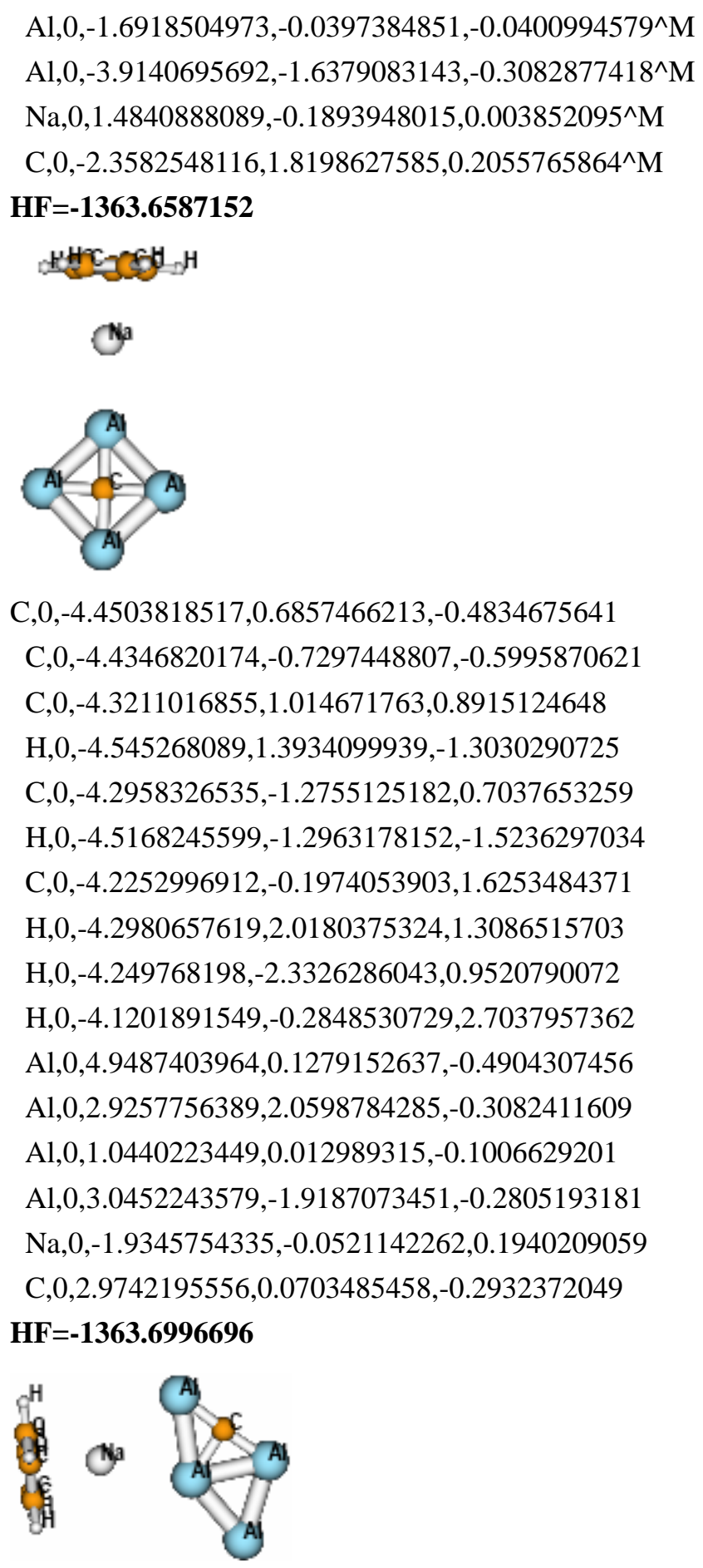

Al,0,1.6299348981,-0.4607795764,-0.0211106778 Al,0,1.2436270061,2.3138667341,0.6385049499 Al,0,4.2779580561,0.0655372493,0.260884086 Al,0,3.3988176341,-2.5267074793,-0.4255768015 C,0,2.724182453,1.1382951509,0.4350048242 Na,0,-1.4842440815,0.1898657496,-0.0385730492 C,0,-4.0057137696,-0.0855999606,0.9725600362 C,0,-4.0440540571,1.097430847,0.1882508063 C,0,-3.8495247724,-1.19041614,0.0937641486 
H,0,-4.0809524915,-0.1362628253,2.0560398083

C,0,-3.9107680522,0.7238596952,-1.1749165833

$\mathrm{H}, 0,-4.1431864431,2.1116376405,0.5657489574$

C,0,-3.7906461652,-0.6903658042,-1.2329673254

H,0,-3.7770518262,-2.2348013049,0.3859297735

H,0,-3.8993202849,1.4022577585,-2.0244112296

$\mathrm{H}, 0,-3.6680466041,-1.2854973031,-2.1342994442$

\section{$H F=-1363.6587184$}

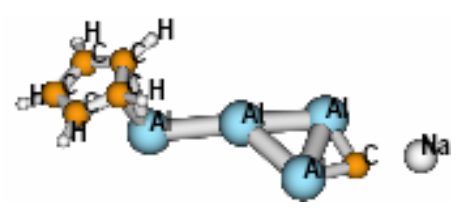

C,0,-3.3443601075,0.6502400453,-0.8455992508 C,0,-3.3616522705,-0.7815962561,-0.881196255 C,0,-4.4882503072,-1.2142708788,-0.1320345345 C,0,-5.1673933393,-0.0745115181,0.3509212135 C,0,-4.4607238346,1.0715280418,-0.0741359639 H,0,-2.6586252908,1.2896433718,-1.3909806825 H,0,-2.6890393671,-1.4088610322,-1.4563010832 $\mathrm{H}, 0,-4.7583320556,-2.2482951918,0.0573465658$ H,0,-6.0204670276,-0.0811797875,1.0220885463 H,0,-4.7063830087,2.1009192008,0.1664578044 Al,0,-2.1135774309,-0.1155748403,1.0699768535 Al,0,0.5262188459,-0.0522148586,-0.1132890972 Al,0,2.6478331483,1.6756199718,-0.062682894 Al,0,3.0509899923,-1.0122558012,-0.1817222913 $\mathrm{Na}, 0,6.1184680601,-0.6670972044,0.1594674479$ C,0,4.1691563366,0.5558396584,0.0133070405 HF $=-\mathbf{1 3 6 3 . 5 7 3 5 2 9 9}$
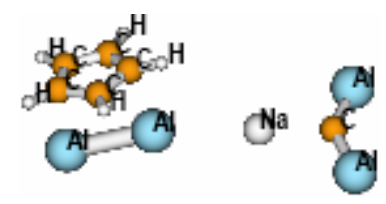

C,0,-4.1477356083,1.1405025394,-0.0862524873 C,0,-4.3933967675,0.0384812634,0.7738966897 C,0,-2.835791006,1.6150879851,0.1657782458 H,0,-4.8523181138,1.5649116775,-0.794450168 C,0,-3.213637764,-0.1839182321,1.536845728 H,0,-5.3292322142,-0.4994183149,0.8724353226 C,0,-2.2635629023,0.8236176354,1.2003775533 H,0,-2.3673978339,2.4702904157,-0.3109887425 H,0,-3.0915993242,-0.9349906685,2.3108111535 H,0,-1.3270655428,1.0157759846,1.7087469044 Al,0,5.0373900799,1.633216808,-0.6155021126 
Al,0,-1.1844788013,-0.5178550206,-0.5645416657

Al,0,5.1604174891,-1.5702110181,0.7577374561

Al,0,-3.7760740345,-1.2302063271,-1.35802977

Na,0,2.1195500191,-0.1994348878,-0.2038726394

C,0,4.4488325957,-0.019948371,0.0094232309

HF $=-\mathbf{1 3 6 3 . 5 8 3 1 1 8 6}$

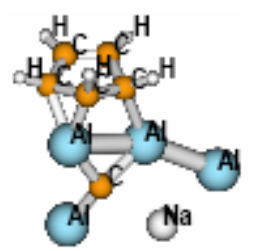

C,0,1.5608762799,2.8848206775,0.1929598936

C,0,1.9997067166,1.9446953353,-0.8730015622

C, $0,0.2125866466,2.8553866729,0.3327834174$

H,0,2.2388914944,3.4613889535,0.824237061

C,0,0.6801509895,1.6513177789,-1.7201720816

H,0,2.8782672448,2.229067253,-1.4648276539

C,0,-0.4017060829,1.8206434684,-0.5864182233

$\mathrm{H}, 0,-0.3537154245,3.4007854916,1.0875733127$

H,0,0.5157003617,2.2918184381,-2.600217932

H,0,-1.3782845828,2.1147720592,-0.9986486

Al,0,-3.1309498359,-0.9123706458,1.7604349109

Al,0,-0.9123570954,0.04346988,0.5152308835

Al,0,1.6527479785,-2.5678656004,0.8141792627

Al,0,1.3524863361,0.0327019559,-0.9396814343

Na,0,-1.5940801439,-2.5638935844,-0.8322730285

C,0,0.4698798682,-1.3305581719,0.046308561

HF $=-1363.5549635$

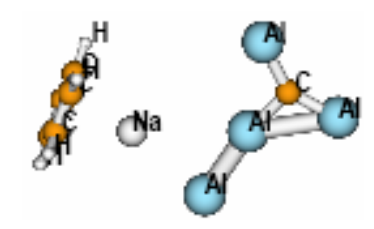

C,0,0.7617276204,3.7439158105,-1.2872893543

C,0,1.3178501647,2.9035003289,-2.2869244374

C,0,-0.574984353,4.0452864229,-1.6609492384

$\mathrm{H}, 0,1.2701906791,4.0918060579,-0.3917705481$

C,0,0.3247154309,2.6856538969,-3.278350771

H,0,2.3242010911,2.4935375034,-2.2882622547

C,0,-0.8447469471,3.3915912527,-2.8913580557

H,0,-1.2721952585,4.6627355814,-1.0999409089

$\mathrm{H}, 0,0.4394804908,2.0820658237,-4.1754231658$

H,0,-1.7864064105,3.4148675502,-3.4338476236

Al,0,0.0813617057,-4.2552205094,2.9638024543

Al,0,-2.65614209,-0.8608452753,0.1263805572 
Al,0,-0.4758388697,-1.8291566451,1.1798897927

Al,0,2.5723580614,-1.9359049498,1.781540163

$\mathrm{Na}, 0,-0.5569113806,1.3489333873,-1.0354609501$

C,0,0.9101297671,-2.7913850188,2.0895962531

\section{HF $=-\mathbf{1 3 6 3 . 6 4 9 4 4 7 5}$}

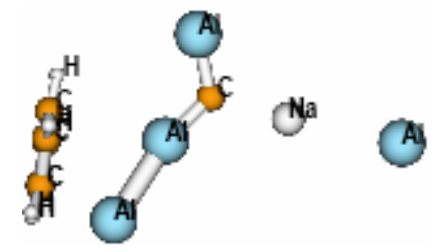

C,0,3.468356978,0.3754004136,1.2514871425 C,0,3.857556495,-0.8935335968,0.7550941577 C,0,3.2876953625,1.2494266366,0.1574846552 H,0,3.2974922132,0.6192933983,2.2957508164 C,0,3.9050617784,-0.799081677,-0.678390284 H,0,4.2111477529,-1.7318198166,1.3484922197 C,0,3.5458161023,0.5289752738,-1.0274951677 H,0,2.8997356293,2.2617569948,0.2120915836 H,0,4.3211673735,-1.5448218481,-1.3497358615 H,0,3.4498740437,0.9112961233,-2.0393072785 Al,0,1.919126719,-2.1596698764,-0.1642469114 Al,0,-6.737231366,-1.2329679468,0.0867459201 Al,0,0.0823066747,-0.1022590339,-0.066746168 Al,0,-1.3311416329,2.818116575,-0.0743667177 $\mathrm{Na}, 0,-3.5652991256,-0.0201653965,-0.0034778849$ C,0,-1.4129720101,0.9561893126,-0.0560228944 $H F=-1363.5572146$

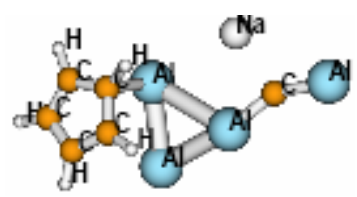

C,0,-3.5621926425,-0.8092868946,0.6646782595 C,0,-2.324520642,-0.4473363897,1.221434581 C,0,-4.1221769082,0.3360524861,0.0357695378 H,0,-3.9806152324,-1.8115568127,0.6507421544 C, $0,-2.0908624628,0.9447988599,0.9240304656$ H,0,-1.6786946939,-1.0748493262,1.828498109 C,0,-3.2457630753,1.4152969998,0.2136503312 $\mathrm{H}, 0,-5.0435495182,0.3541885505,-0.5412734271$ H,0,-1.3987324971,1.5802464483,1.471568344 H,0,-3.3837306616,2.426813632,-0.1566923373 Al,0,-0.6557407114,0.7994564917,-0.9356396983 Al,0,4.920572681,-0.1331535181,0.6869926337 
Al,0,-0.845017924,-1.8742305642,-1.0248257034 Al,0,1.5012590201,-1.0726082785,0.0535342256 $\mathrm{Na}, 0,2.2599974226,1.8887801821,-0.6334599707$ C,0,3.1207492475,-0.2069347608,0.2028398057 HF $=-\mathbf{1 3 6 3 . 5 9 4 3 2 6 5}$

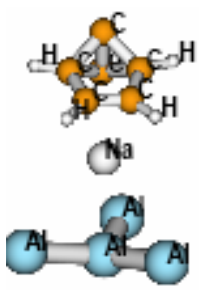

C,0,-3.3283727377,-0.3046922705,0.3385442388 C,0,-2.8666555187,-0.2271544763,-1.0884089054 C,0,-2.4604859881,-1.2939596617,1.1051706382 $\mathrm{H}, 0,-3.8097485733,0.5361921136,0.8349333528$ C,0,-2.3454001684,-1.4465978924,-1.3605161194 H,0,-2.9507966071,0.632644746,-1.7472744282 C,0,-2.5034200571,-2.2347000381,-0.0918015904 H,0,-1.9573650215,-1.2968655125,2.0811508682 H,0,-1.908975443,-1.8010822388,-2.290136847 H,0,-2.1823005127,-3.2720957169,-0.0138873035 Al,0,2.5447505548,1.0784597907,0.0375501676 Al,0,2.7755689775,-0.1451057525,2.2747877775 Al,0,2.7201225908,-0.3069828476,-2.1077505636 Al,0,1.2069085188,3.2603078454,0.0140484494 $\mathrm{Na}, 0,-0.3222112814,-0.1460904662,0.059656525$ C,0,-3.8060068784,-1.7793332828,0.7031328682 $H F=-1363.4850767$ 

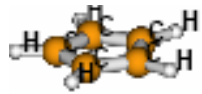

c

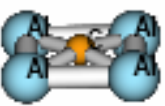

C,0,3.6875718935,0.0900536027,-1.2085334484

C,0,3.684849563,1.1798014902,-0.2991827434

C,0,3.6887834335,0.6517106179,1.0182651209

C,0,3.6934976859,-0.76444907,0.9231509332

C,0,3.6922097104,-1.1115518618,-0.4531129327

H,0,3.6949044843,0.1630836444,-2.2936574007

H,0,3.6904251351,2.2343449268,-0.5652575281

H,0,3.6975338272,1.2306036129,1.9389575467

H,0,3.7056412803,-1.4611827568,1.7581791586

H,0,3.7034720853,-2.1210100917,-0.8577473778

Al,0,-2.521774021,1.6444835834,1.1095833732

Al,0,-2.5236635956,1.1016977539,-1.6476360218

Al,0,-2.5161833457,-1.6556260088,-1.1049050632

Al,0,-2.5142284429,-1.1128126152,1.6524669241

$\mathrm{K}, 0,0.8324184164,0.0000662981,-0.0000358476$

C,0,-2.3338930291,-0.005190491,0.0021775615

HF $=-1801.3037692$

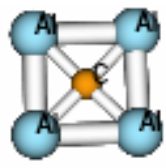

(6)

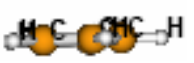

Al,0,1.4231136808,-4.6056180203,-0.0140729675 Al,0,1.4196337017,-1.8254605859,-0.012799009 Al,0,-1.4005811912,-1.835634801,0.0119596909 Al,0,-1.3843968073,-4.6155497628,0.0106437836 $\mathrm{K}, 0,-0.0039132293,1.5835102444,0.0007162128$ C,0,1.1855848882,4.4786376983,0.0053798079 C,0,0.3551460772,4.4752149651,-1.1453740335 C,0,0.3475088691,4.4743514119,1.1505971312 $\mathrm{H}, 0,2.2730810536,4.4907858766,0.008986335$ C,0,-0.9962730769,4.4688856388,-0.7112886682 $\mathrm{H}, 0,0.6951153767,4.4886893197,-2.1786203658$ C,0,-1.0010072053,4.4683745814,0.7075668803 H,0,0.6806290144,4.4872013326,2.1860882401 H,0,-1.8744507587,4.4740474274,-1.3529831577 
H,0,-1.8834025761,4.4730540803,1.3434424504

C,0,0.0144366568,-3.2039728739,-0.0010529544

\section{$\mathrm{HF}=-\mathbf{1 8 0 1 . 3 1 1 2 7 2 6}$}

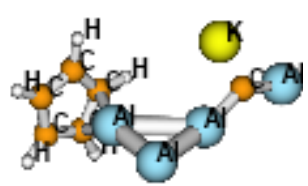

C,0,2.0967342086,-1.5602015221,0.6792013051

C,0,2.8146349119,-1.713364483,-0.5416311025

C,0,3.8835596722,-0.7775269368,-0.5433327077

C,0,3.8275966099,-0.0417489848,0.6648821638

C,0,2.7235359223,-0.5171249214,1.420169947

H,0,1.2201217026,-2.1209921662,0.9802253896

H,0,2.5711693727,-2.4094654122,-1.3353958071

H,0,4.5789811869,-0.6116172291,-1.3580512205

$\mathrm{H}, 0,4.4713465095,0.7871009534,0.9362644641$

H,0,2.3986565336,-0.1419544981,2.3834994771

Al,0,1.5648624143,0.3736119179,-0.5551789534

Al,0,-1.3542867469,-0.0029263576,-0.9942686945

Al,0,0.1273714094,2.0336691848,-1.9434196466

Al,0,-3.8258975825,-2.0944870279,0.1076535652

K,0,-2.4085168729,1.6729641119,1.6695337632

C,0,-2.7019110158,-0.6096448347,0.1007261738

HF $=-\mathbf{1 8 0 1 . 2 0 4 3 6 9}$

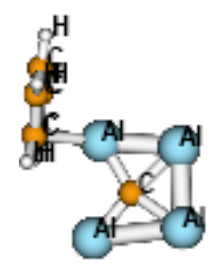

C,0,-3.0789115127,-0.5450007553,-0.5981126762

C,0,-2.9067533425,-1.8339915819,-1.1714143926

C,0,-2.8921174027,-0.6850592502,0.8316072551

$\mathrm{H}, 0,-3.4433600247,0.34638212,-1.0981198255$

C, $0,-2.6301956378,-2.7517800493,-0.1384436256$

$\mathrm{H}, 0,-2.933053629,-2.0561093853,-2.2327401113$

C,0,-2.6161865774,-2.0567354421,1.086144183

$\mathrm{H}, 0,-3.1003334363,0.08357493,1.5687366731$

H,0,-2.3647595616,-3.7946393507,-0.2748329752

$\mathrm{H}, 0,-2.3785722264,-2.4780747676,2.0568333092$

Al,0,-1.4930850033,2.9301920746,0.2239807965

Al,0,1.3517038581,2.9412022363,-0.272695076

Al,0,-0.9124408209,-0.1855614882,-0.1053346263

Al,0,1.5395161956,0.2968717809,-0.3871391902 
K,0,4.9155866609,-1.6978207805,0.385831761

C,0,-0.124184304,1.6029506514,-0.0623199572

\section{HF $=-\mathbf{1 8 0 1} .2538623$}

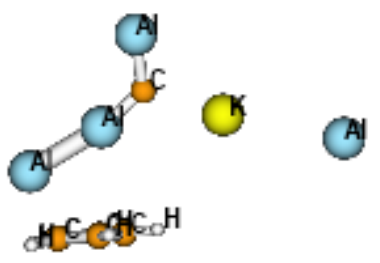

C,0,2.1454763052,-2.3796317289,-0.9548921603 C,0,3.4632635701,-2.0505753057,-0.5501005885 C,0,1.3167777616,-2.3911104502,0.1875046689 H,0,1.8253549401,-2.5578268018,-1.9770678936 C,0,3.4384329351,-1.8442144289,0.8719444004 H,0,4.3547917965,-2.1146155007,-1.1671819705 C,0,2.1051342569,-2.0531190846,1.3079638935 H,0,0.2403553786,-2.5338086485,0.1885174105 H,0,4.3086349942,-1.7338905047,1.5125510009 H,0,1.7501114407,-1.9399842512,2.3280192609 Al,0,3.7659596835,0.3914070767,-0.1566417592 Al,0,-6.4397821754,-1.1735722855,0.8561072291 Al,0,1.1199015221,1.1438288346,-0.3107810551 Al,0,-0.7729952895,3.7389805974,-0.8536625535 $\mathrm{K}, 0,-2.8377642757,0.5359473047,0.1294488131$ C,0,-0.5210541522,1.9501096675,-0.4123617901 HF $=-\mathbf{1 8 0 1 . 1 5 7 9 7 9}$

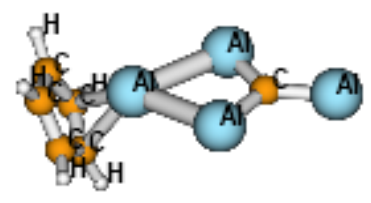

C,0,-2.6277070316,-0.0531145283,-0.2989272101 Al,0,-1.1213377553,1.0311857042,0.1453094585 Al,0,-2.0000474533,-1.7110038801,-1.0185403159 Al,0,-4.4581748639,0.3829181795,-0.0812488958 C,0,2.9202985247,-2.1190156697,-1.0332222806 C,0,3.2236792371,-0.9235181113,-0.3188474658 C,0,2.1977657755,-2.9772138568,-0.16845246 H,0,3.1768478383,-2.3250557452,-2.0658156447 C,0,2.6833659024,-1.0433480964,0.9840266979 H,0,3.7516057161,-0.0617955249,-0.7099737311 C,0,2.0299333334,-2.3058413083,1.0746937004 H,0,1.7806629005,-3.9420465737,-0.4299668463 
H,0,2.7196436747,-0.2865557257,1.7585641872

H,0,1.5075479308,-2.6916889553,1.9422529064 Al,0,0.7642293224,-1.0187452504,-0.504261568

$\mathrm{K}, 0,0.6894198548,4.3654126399,0.8964710743$

\section{HF $=-\mathbf{1 8 0 1 . 2 5 4 7 8 3 8}$}

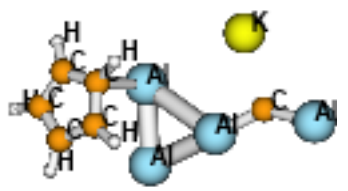

C,0,3.7637750813,-0.9635241179,-0.5330898042 C,0,2.535470792,-0.6650317843,-1.1449985585 C,0,4.344510403,0.2513975111,-0.0777121337 H,0,4.1659206218,-1.9595430776,-0.3739501652 C,0,2.3279815102,0.7621861547,-1.0580266793 H,0,1.8815581995,-1.3605212866,-1.6618806194 C,0,3.4938789143,1.3091067215,-0.4198718388 H,0,5.2657052303,0.336831885,0.4926275473 H,0,1.6601305994,1.3213974453,-1.7091929073 H,0,3.6542825598,2.3612032307,-0.2049046785 Al,0,0.9392392527,0.8045945951,0.7833887884 Al,0,-4.6276299673,-1.1609532594,-0.7963303936 Al,0,1.056675249,-1.812536252,1.2868935966 Al,0,-1.2817091907,-1.1464120588,0.0725638579 K,0,-2.4586421824,2.1732572357,0.3537278645 C,0,-2.9720959032,-0.5095119849,-0.2276734262 HF $=-\mathbf{1 8 0 1 . 1 9 7 6 7 0 7}$

C,0,4.272411542,-1.1491755814,0.5953018272 C,0,3.0471651239,-1.5146918812,1.208739108 C,0,2.1386446155,-1.9034223866,0.1823952599 C,0,2.8156536125,-1.7793608234,-1.0649597502 C,0,4.1296207253,-1.3123813397,-0.8070199407 H,0,5.1428799042,-0.7512395177,1.1040109384 H,0,2.8217878664,-1.469674904,2.2673232853 H,0,1.1154244891,-2.2299677794,0.3256456295 H,0,2.3839072617,-1.9712862848,-2.0398181432 H,0,4.8719789154,-1.0606204199,-1.5555984082 Al,0,1.9097065197,2.9298294915,-0.3020950212 Al,0,2.3716821594,0.4418365186,-0.1078054584 Al,0,-0.1751996757,1.5321375359,0.0484053506 
Al,0,-2.5222143736,0.5251273371,0.4138406964

K,0,-5.8499102587,-0.8616518487,-0.4887621428

C,0,-4.0333879038,-0.1279558959,1.3029476686

HF $=-\mathbf{1 8 0 1 . 1 1 4 8 5 9 5}$

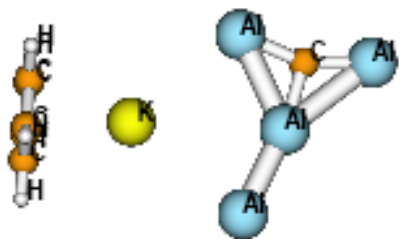

Al,0,-5.4893872047,-0.4734367751,0.1025311948 Al,0,-2.0110364439,-1.7549195574,0.4581958281 Al,0,-2.7943194168,0.9227296316,-0.2206355126 $\mathrm{Al}, 0,-1.2780444548,2.9836414506,-0.7183361752$ C,0,-3.6213398642,-0.7653498966,0.1914663724 K,0,1.3540384315,0.0851241817,-0.0027808752 C,0,4.2721301767,0.0442141624,1.1627504144 C,0,3.9672048287,-1.3317210734,0.9928146855 C,0,4.4548388828,0.6151655988,-0.1235285319 H,0,4.3630862982,0.5667295145,2.1126622851 C,0,3.9605694657,-1.6106187983,-0.3980580353 H,0,3.7766066563,-2.0484071978,1.7883037618 C,0,4.2616373678,-0.4072529314,-1.0882876103 $\mathrm{H}, 0,4.7031524087,1.652997695,-0.333116469$ $\mathrm{H}, 0,3.7645158051,-2.5784620218,-0.8538035134$ H,0,4.3419012491,-0.2910315565,-2.166972562 HF $=-\mathbf{- 1 8 0 1 . 2 5 9 4 9 9 9}$

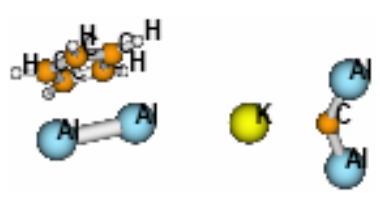

C,0,4.3437704843,0.1914029638,1.3711892589 C,0,4.9981964006,0.5992166176,0.1756342417 C,0,3.0415576536,0.7531517177,1.382749937 H,0,4.7840332454,-0.4020417506,2.1664976461 C, $0,4.0608218961,1.3587394087,-0.5814849734$ H,0,6.0407800382,0.4406944379,-0.0696139266 C,0,2.8674214846,1.4878893448,0.180991425 H,0,2.3171590659,0.6668111741,2.1860689191 H,0,4.2529233119,1.8331072381,-1.5387226646 $\mathrm{H}, 0,2.0048868702,2.0906807021,-0.0795801319$ Al,0,-5.4024388206,1.7425805171,-0.5613499527 Al,0,1.733539127,-0.7108334561,-0.405790849 Al,0,-5.6835499241,-1.4981239408,0.6773203523 Al,0,4.3622858532,-1.5728550524,-0.8851736653 
K,0,-2.1616025796,-0.2520991598,-0.144459293

C,0,-4.8879686829,0.0547078394,0.0300868134

\section{$\mathrm{HF}=\mathbf{- 1 8 0 1 . 1 8 4 2 3 6 4}$}

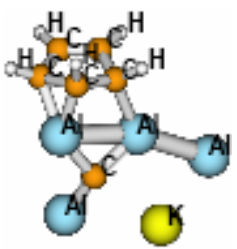

C,0,1.6741022253,3.0927230396,0.2933811811 C,0,2.139703911,2.190039304,-0.7913672409 C,0,0.3234362892,3.0560214553,0.4063197605 H,0,2.3368461026,3.6550049224,0.9523152239 C,0,0.83278126,1.8934769382,-1.6613927576 H,0,3.0180538809,2.5049955114,-1.366601025 C,0,-0.2754242537,2.0478196219,-0.5532628267 H,0,-0.257824437,3.586288103,1.1603077913 $\mathrm{H}, 0,0.6849793713,2.5337544925,-2.5430793282$ H,0,-1.2396098322,2.3600646865,-0.9807800781 Al,0,-2.8689352442,-0.524967832,2.0492134353 Al,0,-0.7546271264,0.2638710301,0.524083944 Al,0,1.7463539333,-2.2817128248,0.9767599338 Al,0,1.5304562446,0.2715388051,-0.9026207044 K,0,-1.6802329154,-2.7496049091,-0.9557745386 C,0,0.6203603701,-1.0919293192,0.0598015062

\section{HF =-1801.1668939}
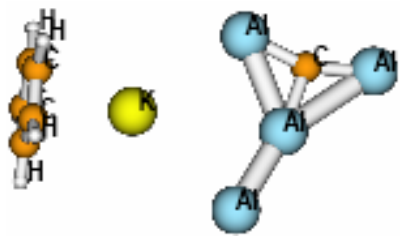

C,0,-4.1281563643,-1.0378129287,-0.8607288944 C,0,-4.0686704302,-1.3555096287,0.520670382 C,0,-4.3154234835,0.3643324174,-0.980918802 H,0,-4.053803601,-1.746658273,-1.6823999127 C,0,-4.2186161956,-0.1497813443,1.2544970078 H,0,-3.9369460295,-2.3492517093,0.9422404491 C,0,-4.3717059044,0.9129831252,0.3263000849 H,0,-4.4101773807,0.91998828,-1.9112639542 H,0,-4.2285556517,-0.0581880802,2.3385183492 H,0,-4.5113412397,1.9628607619,0.5728647475 Al,0,5.4678371752,-0.8372575917,-0.0440762033 Al,0,1.436201706,2.9568910126,-0.0962197576 Al,0,2.8711550717,0.7803789239,-0.0582948155 $\mathrm{Al}, 0,1.9087687428,-1.9219930627,0.0396717627$ 
K,0,-1.3491677249,0.1152100579,0.0170880144

C,0,3.5831549837,-1.0062104321,-0.0129339073

\section{$\mathrm{HF}=\mathbf{- 1 8 0 1 . 2 5 9 5 4 1 8}$}

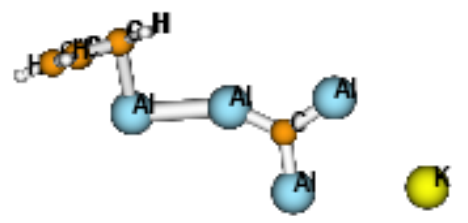

C,0,-4.0433338459,0.8578972097,-0.7256714716

C,0,-4.0347363728,-0.5226551602,-1.0939947181

C,0,-5.1017620171,-1.1583036851,-0.4013519074

C, $0,-5.7697087089,-0.186255088,0.3756663349$

C,0,-5.1153259057,1.0516243102,0.1886148921

H,0,-3.3860826038,1.6220375452,-1.1249266216

H,0,-3.371170646,-0.9794012863,-1.8194445202

H,0,-5.3420523374,-2.2151737737,-0.4489211692

H,0,-6.5817924407,-0.3763521591,1.0695094621

H,0,-5.3683762793,1.9884284775,0.6736093168

Al,0,-2.8916671756,-0.3266506207,0.9811280868

Al,0,-0.1078006493,0.0181209343,-0.1988729934

Al,0,2.8682206106,0.4829947152,-1.5315988627

Al,0,2.6791920237,-0.4208314579,1.5690193195

K,0,6.5698990407,0.1487496835,0.0641639756

C,0,1.7478851856,0.0138558778,-0.0473838228

HF $=-\mathbf{1 8 0 1} .2457863$ 
4

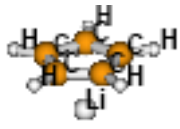

bi

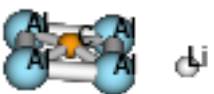

C,0,-2.7122746714,-1.2900090749,-0.1911648829

C,0,-2.6777242495,-0.6303774138,1.067519135

C,0,-2.6698024917,0.7700400571,0.8287149096

C,0,-2.6991931742,0.9756729393,-0.5784708097

C,0,-2.7252415073,-0.2975206708,-1.2083755554

H,0,-2.7459271066,-2.3627369896,-0.348286426

H,0,-2.6764173465,-1.1104696109,2.0399587486

H,0,-2.6684517557,1.5446188054,1.5878172887

H,0,-2.7248186155,1.935344471,-1.0835094767

H,0,-2.7648700007,-0.4796321734,-2.2766813444

Al,0,1.331812393,1.4021955535,1.424276686

Al,0,1.607898015,-1.2994478779,1.4220109592

Al,0,1.7113980282,-1.2895611813,-1.4011507351

Al,0,1.4375677175,1.4166901568,-1.3990135073

C,0,1.2606208463,0.0282851296,-0.0009262172

Li,0,-0.9236604962,-0.1150085182,-0.0570324017

Li,0,1.3676740785,3.7452337547,0.026729489

Li,0,2.145781855,-3.5809141582,0.0227420775

HF $=-\mathbf{1 2 2 4 . 0 2 0 3 5 9 8}$

di

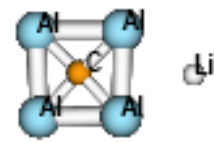

di

HOGHECCH

Al,0,1.3862327596,-3.6894869683,-0.0919592307

Al,0,1.3405553035,-0.8963143358,-0.0629705348

Al,0,-1.3323341714,-0.8878019547,0.0817060061

Al,0,-1.3990836979,-3.6803193127,0.0602844997

Li,0,0.0079604792,1.8624687833,0.0175415776

C,0,1.1954739968,3.6596993065,0.0003112691

C,0,0.3598293591,3.6399813244,-1.1486635952

C,0,0.3613141371,3.661683709,1.1505953962

H,0,2.2799798947,3.6791257703,-0.0005877562

C,0,-0.9912708094,3.6302177857,-0.7083762443

H, 0,0.6944189216,3.6436678635,-2.1805908198

C,0,-0.9903291089,3.6435796854,0.7123058905

H,0,0.6972569554,3.6856434742,2.1818292244

H,0,-1.8694835338,3.622992555,-1.3450882923

H,0,-1.8676282055,3.648643947,1.3503123872

C,0,-0.0011668284,-2.3230011827,-0.0032347548 
Li,0,-3.7170786834,-2.2049845393,0.1992759782

Li,0,3.7152006115,-2.2308091908,-0.2053732811

\section{HF $=-1224.0120246$}

dुi

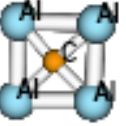

di

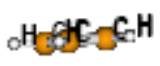

di

Al,0,1.1878519578,-3.8531888413,-0.0198121032 Al,0,1.3577227029,-1.0375526152,-0.0362976654 Al,0,-1.3858438832,-0.9122140122,0.0331335142 Al,0,-1.5848770574,-3.6726134685,0.0509314816 Li,0,-0.0437369087,1.5214168459,-0.0077334175 C,0,1.0943051362,3.619506671,0.0133611923 C,0,0.2723143905,3.5887769053,-1.1518964934 C,0,0.2402636807,3.5786991982,1.1552043344 H,0,2.1773225737,3.6325633107,0.0284632456 C,0,-1.0894859735,3.5325367551,-0.729681412 H,0,0.6223710673,3.5819776111,-2.177384087 C,0,-1.1092372341,3.5264161824,0.6952333171 H,0,0.5618101521,3.563140763,2.1899016351 H,0,-1.9557502226,3.4632670969,-1.3763223761 H,0,-1.9928224,3.4510203682,1.3172169953 C,0,-0.0625584829,-2.3641978008,0.0057292881 Li,0,-0.1902826105,5.33444084,0.0036677458 Li,0,3.5808066413,-2.6558578303,-0.08693257 HF $=-\mathbf{1 2 2 4 . 0 1 9 3 6 8 5}$

bi

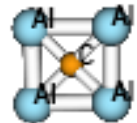

di<smiles>[10BH2][18OH]</smiles>

di

Al,0,1.4367064804,-3.6138812827,-0.0125719147 Al,0,1.4007732069,-0.8876715867,-0.0148793453 Al,0,-1.3788020908,-0.8911833994,0.0105184046 Al,0,-1.4085866486,-3.6169146181,0.0135054278 Li,0,0.0088344549,1.5904749248,-0.0030128048 C,0,1.1937501872,3.6653861182,0.0043764671 C,0,0.3585929084,3.6561058543,-1.1511814292 C,0,0.3521551096,3.651216899,1.1552539103 H,0,2.2765750857,3.6403555667,0.0073089357 
C,0,-0.9995572474,3.6398026549,-0.7141756393

H,0,0.6976228224,3.63489634,-2.1802133883

C,0,-1.0035051876,3.6368429333,0.7107649933

$\mathrm{H}, 0,0.6854536591,3.6259186771,2.1860659649$

H,0,-1.8744152548,3.5957652127,-1.3515709559

H,0,-1.8818211273,3.5901205675,1.3431726932

C,0,0.0126409532,-2.280092074,-0.0009501871

Li,0,-0.0377885521,5.4133282836,0.0044545314

Li,0,0.0159348142,-5.9295262579,0.0036464799

HF $=-\mathbf{1 2 2 4 . 0 2 4 4 1 9 2}$

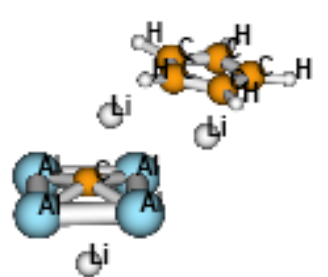

Al,0,-2.6541052676,-1.978880779,-0.0172550863 Al,0,-0.5860116605,-1.1822165937,1.612147487 Al,0,-0.9114056696,1.4761814412,0.7640989711 Al,0,-3.0718590024,0.610567577,-0.8303009302 Li,0,1.8123389475,0.3856559611,1.0262562015 C, $0,2.465741048,-0.8608507703,-0.8868785211$ C,0,2.3117019866,0.5001270286,-1.2967845455 C,0,3.4218292644,-0.8930809419,0.1622835276 H,0,2.0587760558,-1.7462954737,-1.3755555709 C,0,3.1727394801,1.2944121818,-0.495316645 H,0,1.7555178615,0.8681697284,-2.1587527212 C,0,3.8651015728,0.4326829381,0.3925248262 H,0,3.7611691355,-1.7832076581,0.6786202654 H,0,3.2924249089,2.3689299862,-0.5699982086 H,0,4.6088399171,0.7360130256,1.1212759539 C,0,-1.5369529903,-0.2664212318,0.0459064406 Li,0,-2.7035782613,3.2574774141,-0.3211514911 Li,0,0.2135155067,-0.4577427574,-1.0575016733

\section{HF $=-\mathbf{1 2 2 4 . 0 1 9 0 0 0 8}$}

di

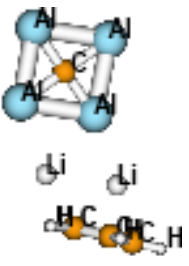

$\begin{array}{rcccl}6 & 0 & 4.010145 & -0.211661 & -1.146855 \\ 6 & 0 & 3.359947 & 0.974854 & -0.715094 \\ 6 & 0 & 3.355511 & 0.976267 & 0.712960\end{array}$




\begin{tabular}{|c|c|c|c|c|}
\hline 6 & 0 & 4.002900 & -0.209403 & 1.151250 \\
\hline 6 & 0 & 4.418738 & -0.932068 & 0.004263 \\
\hline 1 & 0 & 4.186522 & -0.496916 & -2.177531 \\
\hline 1 & 0 & 3.051177 & 1.787465 & -1.371924 \\
\hline 1 & 0 & 3.043524 & 1.790563 & 1.366264 \\
\hline 1 & 0 & 4.172709 & -0.492596 & 2.183589 \\
\hline 1 & 0 & 4.958021 & -1.872918 & 0.006923 \\
\hline 13 & 0 & -4.127613 & 0.386428 & 0.001768 \\
\hline 13 & 0 & -1.619929 & 1.598509 & -0.001125 \\
\hline 13 & 0 & -0.456618 & -0.881203 & -0.003053 \\
\hline 13 & 0 & -2.955849 & -2.134974 & 0.000136 \\
\hline 6 & 0 & -2.278443 & -0.240905 & -0.000498 \\
\hline 3 & 0 & 2.280264 & -0.972575 & -0.003627 \\
\hline 3 & 0 & 1.189008 & 1.429313 & -0.002858 \\
\hline 3 & 0 & -3.984150 & 3.059266 & 0.001845 \\
\hline \multicolumn{5}{|l|}{$H F=-1224.0280961$} \\
\hline \multicolumn{5}{|l|}{ نbا } \\
\hline 6 & 0 & -4.024909 & -0.340221 & -1.149120 \\
\hline 6 & 0 & -4.382796 & -1.094575 & -0.003041 \\
\hline 6 & 0 & -4.023906 & -0.349616 & 1.149078 \\
\hline 6 & 0 & -3.462775 & 0.881938 & 0.718936 \\
\hline 6 & 0 & -3.463284 & 0.887800 & -0.709084 \\
\hline 1 & 0 & -4.177982 & -0.631411 & -2.181888 \\
\hline 1 & 0 & -4.851853 & -2.072404 & -0.006891 \\
\hline 1 & 0 & -4.175819 & -0.649536 & 2.179522 \\
\hline 1 & 0 & -3.210240 & 1.712876 & 1.376656 \\
\hline 1 & 0 & -3.213877 & 1.725072 & -1.360125 \\
\hline 13 & 0 & 3.945293 & 0.949634 & 0.001824 \\
\hline 13 & 0 & 3.097688 & -1.737896 & -0.000536 \\
\hline 13 & 0 & 0.499821 & -0.871142 & -0.003158 \\
\hline 13 & 0 & 1.375970 & 1.783152 & -0.000803 \\
\hline 6 & 0 & 2.221968 & 0.012803 & -0.000901 \\
\hline 3 & 0 & 5.745708 & -1.086081 & 0.003453 \\
\hline 3 & 0 & -1.331126 & 1.492615 & -0.003856 \\
\hline 3 & 0 & -2.247933 & -0.967234 & -0.002180 \\
\hline .0333652 & & & & \\
\hline
\end{tabular}




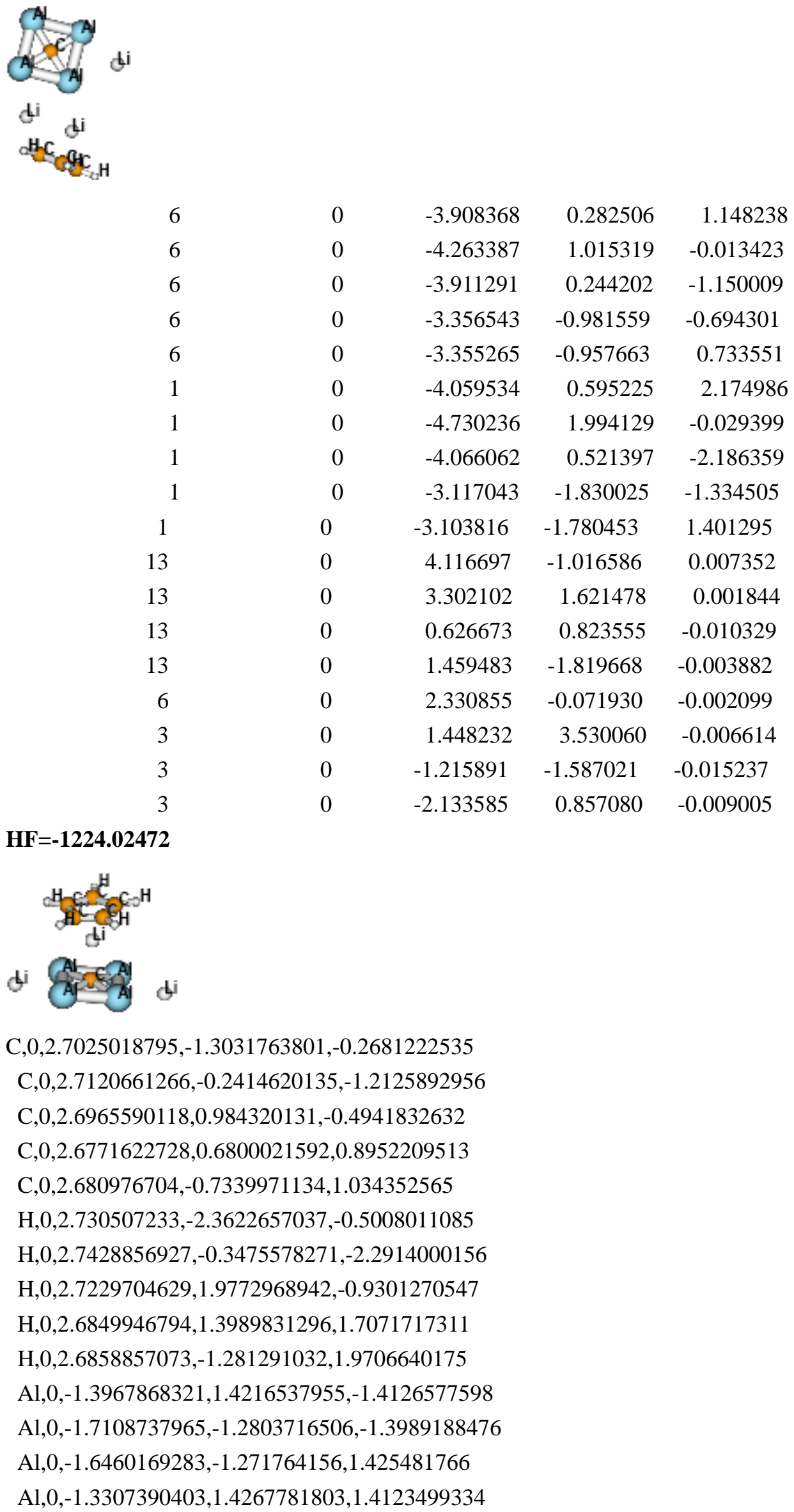


C,0,-1.2631909182,0.0413473035,-0.0012144163

Li,0,-1.3082657417,3.7590161502,-0.0039522187

Li,0,-2.1794422995,-3.5590111964,0.0334348839

Li,0,0.918948551,-0.1330783471,-0.0353524953

\section{$H F=-1224.0203633$}

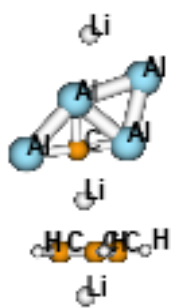

Al,0,2.5977781956,1.110001192,0.59420766

Al,0,0.0726473083,2.3561615533,0.843368889

Al,0,1.4525929418,-1.1093535526,-0.2866551972

Al,0,4.1243982589,-1.1187764211,-0.0986339903

C,0,0.7503367558,0.6151021736,0.2771268986

Li,0,-1.1441225703,-0.0828472738,-0.1106110344

C,0,-3.3315868876,0.5531237124,-0.834351143

C,0,-2.946356039,-0.5627015642,-1.6354903325

C,0,-3.4448481428,0.1078720068,0.5153536019

H,0,-3.4743474086,1.5696080683,-1.1808496778

C,0,-2.8231623019,-1.6975235908,-0.7811403695

H,0,-2.7514251365,-0.5448066102,-2.7012890149

C,0,-3.1295063136,-1.2830135248,0.5482582645

$\mathrm{H}, 0,-3.6878735561,0.7283451317,1.3694882761$

H,0,-2.5075991627,-2.6889410316,-1.0819901242

H,0,-3.0984706589,-1.9083578804,1.4327164705

Li,0,-4.8316557734,-0.9603537636,-0.7066688915

Li,0,5.260457123,1.1607247085,0.7984955431

HF $=\mathbf{- 1 2 2 3 . 9 8 5 8 7 2}$

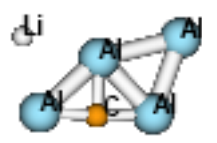

di

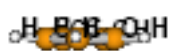

di

Al,0,-2.8370021766,0.7728902195,0.002240054 Al,0,-0.5938941067,2.3669332918,0.0053449375 Al,0,-1.3181533289,-1.4014322714,-0.0147869699 Al,0,-4.097392822,-1.77936045,-0.0040420867 C,0,-0.8665097122,0.4570200626,-0.0036893548 Li,0,1.1444489475,0.0890743554,-0.0084088471 C,0,3.4172665837,0.8658762431,-0.1707378576 
C,0,3.2784874398,0.2158018282,1.0907253038

C,0,3.2304214666,-0.1111660545,-1.1925902068 H,0,3.5906888242,1.9238610115,-0.3251104137

C,0,3.0069039351,-1.1634565734,0.8486088692

$\mathrm{H}, 0,3.3360215828,0.693375761,2.0616745431$

C,0,2.9778998732,-1.3655916356,-0.5623226497

H,0,3.2473925489,0.0732519857,-2.2603071821

H,0,2.8199527513,-1.919091364,1.6022613867

H,0,2.7629391736,-2.3005188793,-1.0655757867

Li,0,4.9078775705,-0.6554611779,0.0146482226

Li,0,-3.0590134357,3.456659487,0.0181825156

HF =-1223.9957694

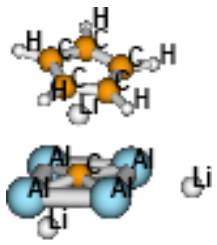

C,0,2.2134763679,0.7657706251,-1.3757493561

C,0,2.4343987996,1.4959092344,-0.1751394719

C,0,2.909920432,0.5894293169,0.8091394398

C,0,2.9836865096,-0.7013393907,0.2194906135

C,0,2.5545156278,-0.592996053,-1.1298886227

H,0,1.8923090543,1.1804037898,-2.3252568411

H,0,2.2936785001,2.5629733281,-0.0400543433

$\mathrm{H}, 0,3.1803918172,0.8402954028,1.8288066197$

$\mathrm{H}, 0,3.321422828,-1.607380873,0.7103027046$

H,0,2.5228635599,-1.4005722809,-1.8534495702

Al,0,-1.607050448,1.5928331194,1.0378333281

Al,0,-1.4469760856,0.3917984506,-1.4891839347

Al,0,-1.3990289415,-2.0996689978,-0.2046563353

$\mathrm{Al}, 0,-1.5200363525,-0.8765166656,2.2653389772$

C,0,-1.2291164947,-0.221524661,0.3654072097

Li,0,0.9464684793,-0.0261324485,0.2041175441

Li,0,-1.7350796913,3.1382689753,-1.1546008235

Li,0,-1.4653086064,-2.011140055,-2.8899246878

HF $=-\mathbf{1 2 2 4 . 0 1 4 7 2 0 1}$

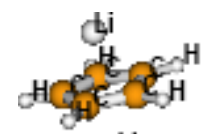

bi

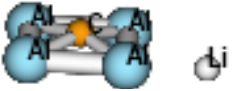

Al,0,1.440403532,-1.667372856,1.3530920239

Al,0,1.4242299416,-1.5962632481,-1.4391402587

Al,0,2.0188174935,1.094848637,-1.4102864547 

Al,0,2.0337613635,1.023065853,1.45412378
C,0,1.3782855788,-0.1391362297,-0.0045545927
Li,0,-0.6678941242,0.1606421122,0.0135765811
C,0,-2.6128960363,-1.0461657658,-0.168022446
C,0,-2.6281267,-0.3855819231,1.0963605717
C,0,-2.6234281476,-0.0484413866,-1.1875583405
$\mathrm{H}, 0,-2.5573674044,-2.1165146741,-0.3260864913$
C,0,-2.6482284599,1.021556143,0.8589548567
H,0,-2.5920480567,-0.8678652303,2.0659285425
C,0,-2.6454617441,1.2300597925,-0.5532723079
H,0,-2.5850954316,-0.2302812414,-2.2549872358
$\mathrm{H}, 0,-2.6400362815,1.7948648956,1.6181255417$
H,0,-2.6369210175,2.1898460813,-1.0564980216
Li,0,-4.4000756299,0.1199511166,0.0005945608
$\mathrm{Li}, 0,2.99025007,3.162935894,0.0694332047$

\section{HF $=-\mathbf{1 2 2 4 . 0 1 9 9 5 8}$}

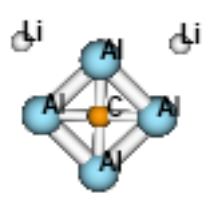
bi

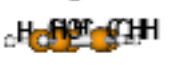

Al,0,2.2076139486,-0.5399485262,1.8891996137 Al,0,0.2732552674,-0.0475474506,-0.0318226492 Al,0,2.2557728589,0.4428330682,-1.9060899139 Al,0,4.1938794648,-0.0459394452,0.0176849751 Li,0,-2.5264064259,-0.0014531222,-0.0506933692 C,0,-4.3435762606,0.2022705165,-1.1901382354 C,0,-4.377863776,-1.0207227238,-0.4672287212 C, $0,-4.258633941,1.2649387416,-0.2508649376$ H,0,-4.3848459592,0.3066974186,-2.2691664094 C,0,-4.314412315,-0.7133786156,0.9189460781 H,0,-4.4525160878,-2.0138893236,-0.8974567177 C,0,-4.2410296774,0.6989184002,1.0526798899 $\mathrm{H}, 0,-4.2242498047,2.3232432917,-0.4871146018$ H,0,-4.3287110391,-1.4309095317,1.7324822419 $\mathrm{H}, 0,-4.1884285497,1.2490408966,1.9862062$ C,0,2.2485921445,-0.0480843739,-0.0082839102 Li,0,4.8309560228,0.6385112096,-2.6044887044 Li,0,4.7632885285,-0.723726028,2.6577660644

\section{HF $=-\mathbf{1 2 2 4 . 0 0 7 4 4}$}




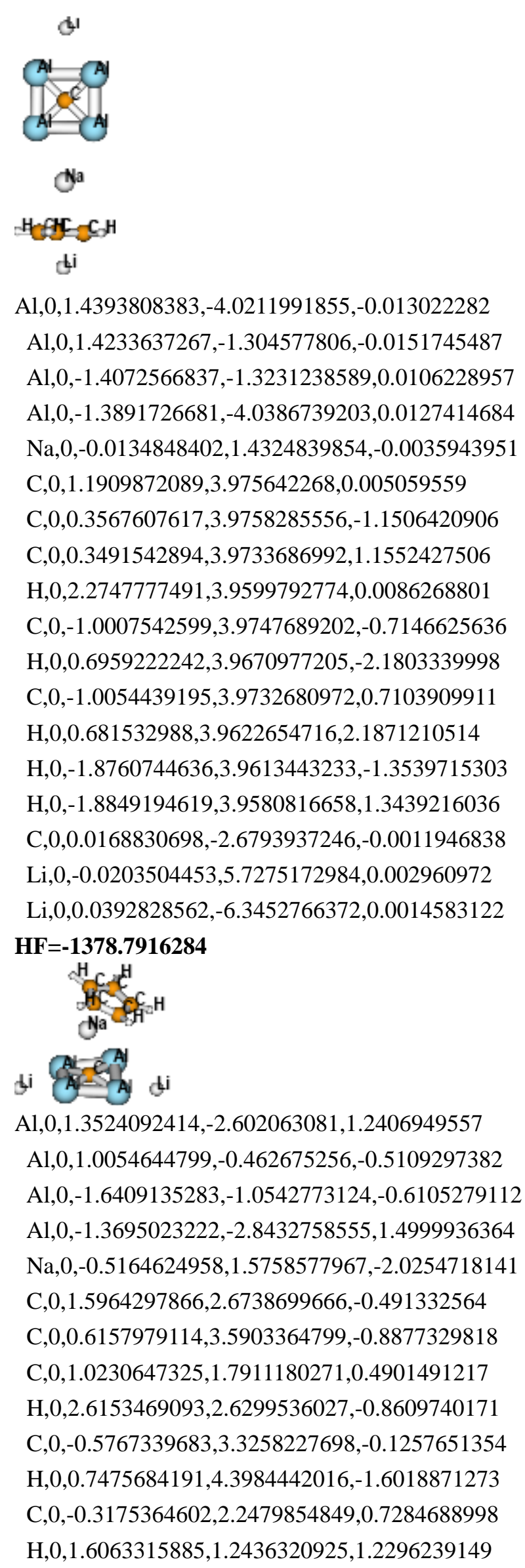


H,0,-1.4995116879,3.897063401,-0.1726115344

$\mathrm{H}, 0,-1.0035014412,1.8256886474,1.4541890371$

C,0,-0.1963768996,-1.6002361006,0.5889986228

Li,0,-3.7525011258,-2.5749504511,0.24168439

Li,0,3.3625113698,-1.7559855191,-0.4533071736

HF =-1378.7555695

bi

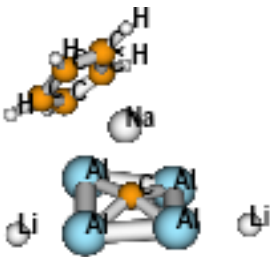

C,0,2.4157790382,-0.4562804525,-1.415618537

C,0,3.0807376038,0.6732544457,-0.9289096132

C,0,3.1928448917,0.5445252248,0.5016576762

C,0,2.5917643818,-0.659571921,0.8801372639

C,0,2.0653996407,-1.3006110086,-0.29857897

H,0,2.2227748981,-0.679849047,-2.4596207961

H,0,3.4844699774,1.4848892398,-1.5277208118

H,0,3.698077697,1.242701525,1.1635867826

H,0,2.5514531897,-1.0662297243,1.8849713484

H,0,1.9923570364,-2.3821599494,-0.3824872689

Al,0,-2.5641202021,1.594190295,0.5469611022

Al,0,-1.9186113302,0.2612498795,-1.7467846114

Al,0,-0.3713265395,-1.5838461961,-0.2854920175

Al,0,-1.4583944567,-0.5944073417,1.9431641062

C,0,-1.2589813605,0.195850796,0.1250158609

$\mathrm{Na}, 0,0.8425140374,1.3785870421,0.0595312843$

Li,0,-3.1828825684,1.1186801419,3.165067132

Li,0,-1.3771739359,-2.3020929055,-2.6543427984

HF $=-\mathbf{1 3 7 8 . 7 6 2 4 0 1 9}$

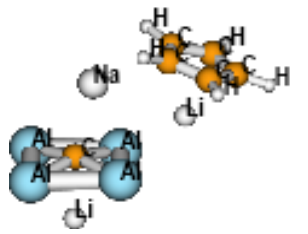

C,0,-3.557886203,-1.00715252,-0.2685908826

C,0,-2.7392477794,-0.8413025383,0.8786530657

C,0,-2.6112748676,0.5593222248,1.1276557822

C,0,-3.3514037035,1.2475503075,0.1316564792

C,0,-3.9413947225,0.2803922419,-0.7218416106

H,0,-3.8473296968,-1.9545685782,-0.7077587743

H,0,-2.397041411,-1.6561774428,1.5148801541 
H,0,-2.1456085338,1.0323769602,1.9908045734 H,0,-3.4571755839,2.3230475316,0.0505278817 H,0,-4.580114014,0.4898702463,-1.5727900205 Al,0,3.2194949198,1.043630353,0.5925513484 Al,0,2.9907263425,-1.699102206,0.2122834338 Al,0,0.7553496905,-1.2964776874,-1.4026396406 Al,0,0.8743051106,1.452109169,-0.947912789 C,0,1.7924843,-0.0990267939,-0.1403841369 Li,0,2.4471681277,3.5523125691,-0.1399224369 Li,0,-1.8183303996,0.1334578491,-1.0078538501 $\mathrm{Na}, 0,-0.0841177193,-0.512057516,1.4744638436$ HF $=-\mathbf{1 3 7 8 . 7 8 3 3 3 0 1}$

C, $,-2.9923803943,-0.172829714,0.3484215258$
$\mathrm{C}, 0,-3.4049316658,0.8081952958,1.2863749513$
$\mathrm{C}, 0,-3.153614985,1.8424591116,-0.7519342559$
$\mathrm{C}, 0,-2.8361494087,0.4699315854,-0.9182753475$
$\mathrm{H}, 0,-2.9426207594,-1.2411966659,0.553920997$
$\mathrm{H}, 0,-3.61253887,0.6331315608,2.3354767131$
$\mathrm{H}, 0,-3.8205825122,2.9890587945,1.0446815589$
$\mathrm{H}, 0,-3.1358302512,2.5952342165,-1.5312838769$
$\mathrm{H}, 0,-2.6423806326,-0.0071333354,-1.8779352594$
$\mathrm{Al}, 0,0.9300037096,0.0828661,1.7482152323$
$\mathrm{Al}, 0,2.5802565591,-1.9538463411,0.9333185822$
$\mathrm{Al}, 0,2.8915295922,-0.6760500754,-1.5903957193$
$\mathrm{Al}, 0,1.2388186241,1.3506263975,-0.7552112193$
$\mathrm{C}, 0,1.6969994284,-0.4818760726,-0.0349054564$
Li,0,-1.4235860384,1.5301769132,0.5997955055
Li,0,4.4915935761,-2.764698822,-0.8460018057
Na,0,-0.6512526353,-1.1626611082,-0.6690396443
HF =-1378.784836




$\begin{array}{rcccc}6 & 0 & -3.534399 & 0.872146 & 1.149015 \\ 6 & 0 & -3.522616 & 1.701809 & -0.000042 \\ 6 & 0 & -3.534478 & 0.872014 & -1.149005 \\ 6 & 0 & -3.574452 & -0.478420 & -0.713566 \\ 6 & 0 & -3.574522 & -0.478333 & 0.713733 \\ 1 & 0 & -3.537903 & 1.205928 & 2.180372 \\ 1 & 0 & -3.498455 & 2.785749 & -0.000103 \\ 1 & 0 & -3.538025 & 1.205679 & -2.180400 \\ 1 & 0 & -3.717954 & -1.336465 & -1.368858 \\ 1 & 0 & -3.718083 & -1.336297 & 1.369115 \\ 13 & 0 & 3.660823 & -1.638890 & 0.000099 \\ 13 & 0 & 3.944969 & 1.144166 & -0.000093 \\ 13 & 0 & 1.167903 & 1.446389 & -0.000155 \\ 13 & 0 & 0.890142 & -1.241381 & 0.000056 \\ 6 & 0 & 2.415778 & -0.066734 & -0.000049 \\ 3 & 0 & 2.856501 & 3.611476 & 0.000476 \\ 11 & 0 & -1.753969 & -2.366999 & -0.000065 \\ 3 & 0 & -1.649062 & 0.636460 & -0.000047\end{array}$

HF $=-1378.790133$

Cு

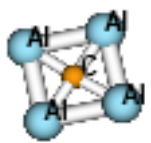

di Na

Heffec-

C,0,-3.9943920961,-0.6953846176,-1.1492999414

C,0,-4.2109549397,-1.4987779199,-0.0004042749

C,0,-3.9931136099,-0.697306442,1.1496969049

C,0,-3.6572198278,0.6116268009,0.7145937349

C,0,-3.657950794,0.6128434683,-0.7121852643

H,0,-4.0927327932,-1.0137176594,-2.1808477366

H,0,-4.4964764663,-2.5449269032,-0.0011538735

H,0,-4.0900557856,-1.0175336549,2.1807922207

H,0,-3.5416232117,1.473912881,1.368379052

H,0,-3.5446767166,1.4765386013,-1.3646011872

Al,0,4.1912473917,0.7489656377,0.0016103172

Al,0,3.1371541558,-1.855049713,0.0049931727

Al,0,0.6135037887,-0.8132070921,0.0000805237

$\mathrm{Al}, 0,1.7184061135,1.8029763075,-0.0033763301$ 
C,0,2.3915294514,-0.041806859,0.0008672165

Li,0,5.8262483765,-1.420172479,0.007425955

$\mathrm{Na}, 0,-1.2836474863,1.8616643593,-0.0078026474$

Li,0,-2.1481652521,-0.9423790622,-0.0005424532

\section{HF $=-\mathbf{1 3 7 8 . 8 0 1 4 9 8 1}$}

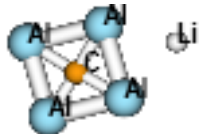

di

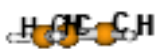

C,0,-3.3373125876,-0.9605565787,-0.5523014366

C,0,-3.3648759817,-1.7807070784,0.6062721152

C, $0,-3.7718992205,-0.9768266758,1.7018351771$

C,0,-3.9807873194,0.3446566306,1.2303207533

C,0,-3.7197755352,0.3581691844,-0.1651180203

H,0,-3.171218228,-1.321180288,-1.5657575162

H,0,-3.1390480952,-2.840463332,0.6390656524

H,0,-3.9028800164,-1.3148752359,2.7238816555

H,0,-4.3075477395,1.1914521564,1.8230439897

H,0,-3.9030093856,1.2048664395,-0.8240541548

Al,0,4.0499019939,2.023181157,-0.7938252986

Al,0,3.2311776468,1.0739267167,1.6598468622

Al,0,0.6944740553,0.6420635622,0.605647995

Al,0,1.5200059297,1.6076079411,-1.8758926659

C,0,2.3399172765,1.3410058693,-0.1289277383

Li,0,3.6262168947,2.645083078,-3.3617601481

$\mathrm{Na}, 0,-1.4294078355,0.6982252223,-1.706778037$

Li,0,-1.8776239423,-0.2353788088,1.0488382954

HF $=-\mathbf{1 3 7 8 . 7 9 5 9 7}$

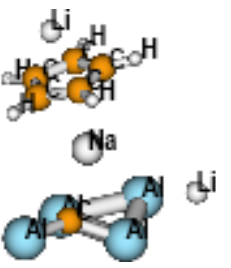

Al,0,2.3387898191,0.2922735697,1.5527212637

Al,0,1.1891044336,2.899383558,-0.2553286095

Al,0,2.5508841833,-0.1472968223,-1.2435537497

Al,0,2.5789073534,-2.1407199457,0.4535717113

C,0,1.5691417325,1.0343667057,-0.0818524746

$\mathrm{Na}, 0,-0.5352426926,-0.2012673885,-0.2267557047$

C,0,-3.0618602456,1.0031405334,-0.0119863259

C,0,-3.2002215323,0.1054291175,-1.1102620873

C,0,-2.9215884787,0.227550153,1.1751387482

H,0,-3.0439515496,2.0852735178,-0.0714744777 
C,0,-3.14630514,-1.2255424598,-0.601430806

H,0,-3.3171190722,0.3863391423,-2.1509623329

C,0,-2.9735941475,-1.1497663167,0.8112611088

H,0,-2.7810345984,0.6171981288,2.1769360942

H,0,-3.2118030701,-2.1357508968,-1.186546596

H,0,-2.8810951531,-1.9917091034,1.4877697566

Li,0,-4.7987858427,-0.1943817585,0.2678829098

Li,0,1.7918940654,-2.6275517709,-2.0822018023

HF $=-\mathbf{1 3 7 8 . 7 4 3 4 1 2}$

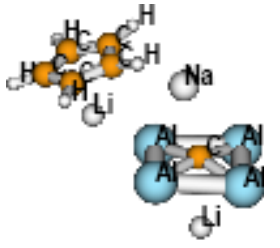

Al,0,-3.2125771778,1.0959033911,0.5442861009 Al,0,-3.0106912133,-1.6574187239,0.2529491319 Al,0,-0.7659930623,-1.3309701761,-1.3610372252 Al,0,-0.8542111604,1.4326707327,-0.9946190064 C,0,-1.7909726691,-0.0821471506,-0.1398819326 Na,0,0.0785415825,-0.4653087821,1.4904443868 C,0,2.7329691137,-0.8424786869,0.9203345817 C,0,3.5543334344,-1.0545323086,-0.2172670642 C,0,2.610360648,0.5669999615,1.1161893133 H,0,2.3857643891,-1.6313587118,1.5859397839 C,0,3.9447128138,0.2135262176,-0.7171864198 H,0,3.8417937982,-2.0189827896,-0.6192011808 C,0,3.3562775075,1.2143402122,0.097451078 H,0,2.1463653151,1.0741010034,1.9607979004 H,0,4.5874333929,0.3882712674,-1.5729968299 H,0,3.467389403,2.2856027914,-0.023196417 Li,0,1.8218933529,0.0628604997,-1.0070171047 Li,0,-2.4131082915,3.5718413874,-0.2678481832 $H F=-1378.7833354$

bi

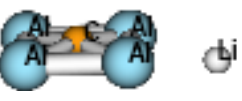

C,0,2.7770001308,1.1555131366,0.6037236467 C,0,2.2865607062,0.7128160995,1.8559943972 C,0,2.2879078938,-0.710136577,1.8560974579 C,0,2.7791255871,-1.1520930305,0.6038726831 C,0,3.0826526481,0.0019355315,-0.1747263494 H,0,2.9222662267,2.1872632866,0.3041526899 
H,0,1.9692956989,1.3504383161,2.6737137268

H,0,1.9718343711,-1.3482187623,2.6739216122

H,0,2.9261434493,-2.1835728261,0.3042436004

H,0,3.6477480207,0.0022063936,-1.1060798787

Al,0,-2.1215454586,-1.4120085021,0.3442596867

Al,0,-2.1256053262,1.4026987644,0.34985537

Al,0,-0.505091,1.4557572937,-1.8209750876

Al,0,-0.5009158627,-1.451691574,-1.8268254481

C,0,-1.0970419066,-0.0013215285,-0.58738127

Li,0,-3.7876552745,-0.0102993869,1.9873534907

$\mathrm{Na}, 0,1.914982249,0.0076206401,-2.8865896591$

Li,0,0.9117368467,-0.0003682747,0.3667623313

HF $=-\mathbf{1 3 7 8 . 7 9 5 3 2 6 3}$

Na

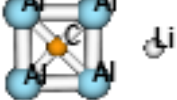

\section{di}

HC. $998 . \mathrm{H}$

C,0,-3.8905585834,-1.4426996892,-0.3187514133

C,0,-3.7425715536,-1.5632481371,1.0892071635

C,0,-3.7819411566,-0.2578346568,1.6484644066

C,0,-3.9554222422,0.669908723,0.5861505154

C, $0,-4.0224363485,-0.062316207,-0.6292894252$

H,0,-3.9124750255,-2.2613197373,-1.0303206106

H,0,-3.6274791784,-2.4898788301,1.6412035967

H,0,-3.7042023325,-0.0131861668,2.7025061468

H,0,-4.0319443605,1.7473507721,0.6866857482

H,0,-4.1591461591,0.3577979871,-1.6200323289

Al,0,3.3150559294,1.1043624098,-0.9143484023

Al,0,3.5641059969,-1.0107784363,0.8524182999 Al,0,0.7904020317,-1.2166796892,0.9809263805 $\mathrm{Al}, 0,0.4870074492,0.8166604814,-0.718230298$ C, $0,2.0655696531,-0.0620244731,0.0368148848$ Li,0,-2.1036877982,-0.4025096804,0.2988603342 $\mathrm{Na}, 0,1.5084361464,2.9914315429,-2.511399321$ Li,0,2.360748645,-2.9153457565,2.4210781483 $\mathrm{HF}=-\mathbf{1 3 7 8 . 7 8 8 2 8 4 8}$

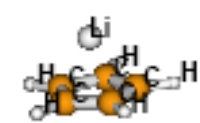

Na

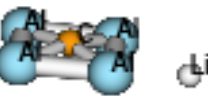

C,0,3.1320445647,-0.7504627862,-0.8232671759

C,0,3.1033637245,0.6466664123,-1.1060689418 
C,0,3.073067472,1.3470536709,0.1360178141

C,0,3.0830540809,0.3820789569,1.1862019041

C,0,3.1197945838,-0.913827345,0.5928889856

$\mathrm{H}, 0,3.1444661961,-1.5491585108,-1.5561166524$

H,0,3.0973785613,1.0964963753,-2.0925612666

$\mathrm{H}, 0,3.0434881785,2.4238407759,0.2603549235$

H,0,3.0567486938,0.5952324265,2.2489130392

H,0,3.1202876173,-1.8582910495,1.1249759936

Al,0,-2.4045999508,1.0549021135,-1.4406588191

Al,0,-1.8209968815,-1.6497290088,-1.3900243763

Al,0,-1.7967153055,-1.6220869775,1.4235771718

$\mathrm{Al}, 0,-2.3790231708,1.0823982058,1.4317622219$

C,0,-1.8357191151,-0.1665944278,0.0022902526

Li,0,4.8588268456,0.1814660647,0.0187671898

Na,0,0.6237554189,0.0518133133,-0.0216908319

Li,0,-3.2454774144,3.2189173756,-0.017392022

HF $=-\mathbf{1 3 7 8 . 7 7 9 9 0 1 6}$

C,0,3.5466580958,1.150445735,-0.033259037

C,0,2.7367325899,0.7185175737,1.0483435552

C,0,2.7361704847,-0.7105097957,1.0522510683

C,0,3.5457336071,-1.149027447,-0.0268799614

C,0,4.05117773,-0.0012845517,-0.6887852156

H,0,3.751150415,2.180930398,-0.2990698805

H,0,2.3138564875,1.378142826,1.8047837792

H,0,2.3116206683,-1.3656073617,1.8116668644

H,0,3.7491374895,-2.1811157218,-0.2872340101

H,0,4.71503537,-0.0039217865,-1.5463960775

Al,0,-3.0419669853,-1.4239678514,0.2483916345

Al,0,-3.0438481158,1.4217493745,0.2396661684

Al,0,-0.7082435611,1.4075401064,-1.2029896211

Al,0,-0.7061080143,-1.4153391579,-1.1940406257

C,0,-1.7530946702,-0.0016004006,-0.1982874368

Li,0,1.9239425654,-0.0016292511,-1.0117892363

$\mathrm{Na}, 0,0.1296902595,0.0080121211,1.484846195$

Li,0,-5.2391070698,-0.0002309151,1.0382176077

HF $=-\mathbf{1 3 7 8 . 7 8 4 8 3 6}$ 


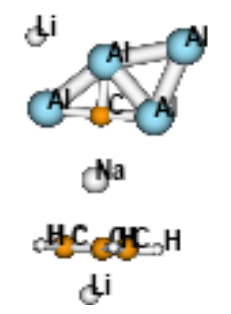

Al,0,3.2737529426,0.6821836182,0.0197132133 Al,0,1.10787562,2.3890338712,-0.0185893286 Al,0,1.6277248022,-1.4068666342,-0.0324303111 Al,0,4.3906021422,-1.9185387695,0.0223148314 C,0,1.2841790135,0.4706638291,-0.0219270332 $\mathrm{Na}, 0,-1.110740759,0.1667675244,-0.0191652123$ C,0,-3.7775122663,0.7642423922,-0.5844941562 C,0,-3.5590933672,-0.5124109991,-1.18059856 C,0,-3.7376487252,0.6017000479,0.8310194285 H,0,-3.9312512452,1.6976855161,-1.1139172345 C,0,-3.3848448041,-1.4636518795,-0.1335127468 H,0,-3.5205631053,-0.7214480201,-2.2437653706 C,0,-3.494606995,-0.7753604275,1.109595786 H,0,-3.8564796567,1.3900736482,1.5656706799 H,0,-3.1828567514,-2.5210946945,-0.2601693027 H,0,-3.3967678606,-1.2194983553,2.0937514842 Li,0,-5.3184856717,-0.5768290884,0.0219440512 Li,0,3.6264217526,3.3545571698,0.0332697835

\section{HF $=-\mathbf{1 3 7 8 . 7 5 3 9 7 2 7}$}

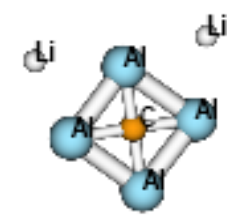

\section{Na

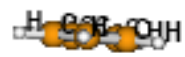

Al,0,1.3699853374,-3.1423406843,-0.8456237779 Al,0,0.1472907788,-0.7846123014,-0.0989678731 Al,0,-2.0269232562,-2.1947396461,0.8697390068 Al,0,-0.7984017543,-4.5771745553,0.1272806446 $\mathrm{Na}, 0,0.4780599344,2.3202123383,-0.0785776169$ C,0,1.7025891194,4.5347440718,0.3515498836 C,0,1.268437761,4.5817600891,-1.001163358 C,0,0.5497531585,4.5565622878,1.1838037943 $\mathrm{H}, 0,2.734561283,4.5345341594,0.6904375597$ C,0,-0.1522894323,4.6324489633,-1.0053044637 H,0,1.9107395758,4.6234467456,-1.8762453334 C,0,-0.5962601687,4.6169736285,0.3451397036 
H,0,0.5470415593,4.5743450601,2.2698797087

H,0,-0.7852245793,4.717804662,-1.8838986359

$\mathrm{H}, 0,-1.6277810834,4.6859835818,0.6783138904$

C,0,-0.3335480326,-2.6935411181,0.0164257368

Li,0,-3.3053397859,-4.4990083828,1.3536350163

Li,0,1.4168548393,-5.8166129612,-1.0331034125

HF $=-\mathbf{1 3 7 8 . 7 5 6 2 2 8 9}$

ن

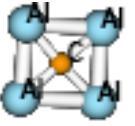

Na

Hesoger

di

Al,0,-4.367468505,0.6900812971,0.1177348257

Al,0,-1.5945346528,1.1491926118,0.2079586352

Al,0,-1.2090749129,-1.5777225976,-0.2971838222

Al,0,-3.9329156208,-1.9836299209,-0.3784862656

Na,0,1.3531375052,0.0180300469,-0.0018767689

C,0,3.827544378,1.3671544196,-0.0669098671

C,0,3.8689608671,0.5915673045,1.1282884855

C,0,3.8600525064,0.4703524256,-1.1746538051

H,0,3.7669630268,2.4480426974,-0.1234153421

C,0,3.9280480419,-0.7842137151,0.7588516991

H,0,3.8485491199,0.9795897289,2.1404559638

C,0,3.9228902317,-0.8589649682,-0.664072336

H,0,3.8317866688,0.7498746029,-2.221824263

H,0,3.9559705122,-1.6271367094,1.4399178887

H,0,3.942373749,-1.7683630439,-1.2535773701

C,0,-2.7505411784,-0.3819771291,-0.078440135

Li,0,5.6347789911,0.2344139278,-0.0168295516

Li,0,-3.4411294301,3.1582597872,0.5790961036

HF $=-\mathbf{1 3 7 8 . 7 8 6 2 7 5 8}$

di

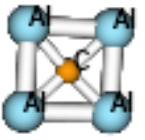

Na

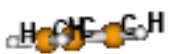

di

Al,0,1.2930408853,-4.1706594436,-0.017300878

Al,0,1.4933582965,-1.3660633971,0.0040883363

Al,0,-1.3039710299,-1.2474491845,-0.0075565652

Al,0,-1.4555569815,-3.9983894381,-0.028540445

$\mathrm{Na}, 0,0.0686741355,1.457837332,0.0143257083$ 
C,0,1.1626466305,4.055020683,-0.0020826458

C,0,0.321194386,4.0014419604,-1.1515583092

C,0,0.3304212131,4.0137338926,1.1545866877

H,0,2.2455925183,4.1037070322,-0.006689547

C,0,-1.0307588364,3.9277012597,-0.7051172146

$\mathrm{H}, 0,0.6522284073,4.0052316044,-2.1839608024$

C,0,-1.0250711747,3.935316988,0.7197237637

H,0,0.6696887881,4.0290270843,2.1842176932

H,0,-1.9085516993,3.8585059686,-1.337301302

$\mathrm{H}, 0,-1.8978570264,3.87337621,1.3595689065$

C,0,0.0533507233,-2.6656383687,-0.0118164747

Li,0,-0.1459737017,5.7387029222,-0.0063281041

Li,0,3.7038891359,-3.0083051254,0.0021621422

$\mathrm{HF}=-\mathbf{1 3 7 8 . 7 8 6 2 7 6 5}$

di
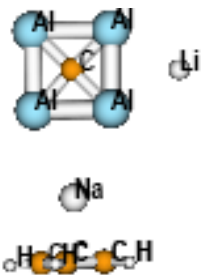

Al,0,1.3817104537,-4.0062827459,-0.088076069 Al,0,1.3514333638,-1.2163487134,-0.0784325846 Al,0,-1.3415728864,-1.2114846768,0.0846103531 Al,0,-1.3825049638,-4.0014448068,0.0788773748 $\mathrm{Na}, 0,0.0165822083,1.8968447168,0.0041693365$ C,0,1.1998404433,4.1765201575,0.0026755526 C,0,0.3668032712,4.1654452054,-1.1491195681 C,0,0.3620248818,4.1699131834,1.1510860208 $\mathrm{H}, 0,2.2846598281,4.2310359045,0.0048873453$ C,0,-0.9861238528,4.1524938878,-0.7124522165 $\mathrm{H}, 0,0.70377387,4.2117129411,-2.1806973454$ C,0,-0.9891184574,4.1552689934,0.7089111633 $\mathrm{H}, 0,0.6947238215,4.2209447073,2.1838346572$ H,0,-1.8631997529,4.1856125609,-1.3523216843 H,0,-1.8688036995,4.1912346246,1.3450412488 C, $0,0.0023049387,-2.6259896173,-0.0003785344$ Li,0,-3.7225703212,-2.5600745008,0.2231574273 Li,0,3.7273025607,-2.5750759077,-0.2270472259 HF $=-\mathbf{1 3 7 8 . 7 6 0 9 3 6}$ 


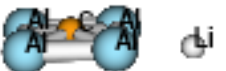

C,0,-2.8295224238,-1.0697907602,0.7765670283 C,0,-2.0090390074,-0.6408955798,1.8526959774 C,0,-1.9524662969,0.7823988586,1.82077748847 C,0,-2.7382961392,1.2264372676,0.725105014 C,0,-3.2812567474, $0.0831386832,0.0840587851$ $\mathrm{H}, 0,-3.0730535241,-2.0983916111,0.5361518672$ H,0,-1.5761515528,-1.3010339446,2.5997305999 H,0,-1.4672745664,1.4388831571,2.5383095632 H,0,-2.8999454328,2.2593251065,0.4385071861 H,0,-3.9325306264,0.0895805841,-0.7830156077 $\mathrm{Al}, 0,1.5948630959,1.3248198486,-0.0989027845$ Al,0,1.4808106624,-1.5479289276,-0.0344705218 Al,0,0.4327000006,-1.538241216,-2.5433478393 $\mathrm{Al}, 0,0.5448193652,1.285913335,-2.6066897291$ C,0,0.7630423239,-0.1066703545,-1.2204131621 Li,0,-1.1320904809,-0.0060019265,-0.0867033642 Li,0,-0.1165051857,-0.1525070171,-4.8208518744 K,0,1.1729665356,-0.0269825032,3.0582513772 HF $=-\mathbf{- 1 8 1 6 . 4 0 7 9 5 3}$

di
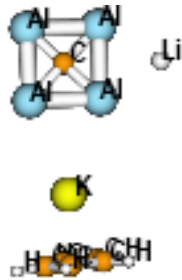

Al,0,0.4622656151,-4.7265763447,-0.7575790521 Al,0,0.8322880014,-1.9594796643,-0.5670856723 Al,0,-1.762402835,-1.6462689555,0.1538170291 Al,0,-2.1645076116,-4.4087720757,-0.0264645499 $\mathrm{K}, 0,0.0062636133,1.8465838408,-0.0210008343$ C,0,1.3486415698,4.4752429272,-0.1407336401 C,0,0.3716576192,4.5435600042,-1.1691577846 C,0,0.6728007209,4.4934781635,1.1083316244 H,0,2.4262679238,4.4753420129,-0.2836723435 C,0,-0.9077768024,4.6045696995,-0.5559355126 $\mathrm{H}, 0,0.5716258758,4.6048631458,-2.2359517754$ C,0,-0.7217729659,4.5734479079,0.851533322 H,0,1.1432760581,4.5111898455,2.0882206232 H,0,-1.8577885894,4.7195004343,-1.0717246194 H,0,-1.5045247506,4.6608442313,1.6008394115 
C,0,-0.6579306637,-3.1903223212,-0.29814422282

Li,0,-4.2229264051,-2.7487710918,0.6891471859

Li,0,2.9050222463,-3.6152091395,-1.2962350036

HF $=-\mathbf{1 8 1 6 . 3 6 7 4 9 5 5}$

di

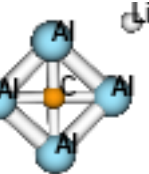

C

Hefea GHH

Al,0,-3.2292112284,1.9585439758,-0.0061833061 Al,0,-1.2849637248,-0.003158701,-0.0401047781 Al,0,-3.233985779,-1.9583830847,-0.0067975484 $\mathrm{Al}, 0,-5.2065246301,0.0016627632,0.029655531$ $\mathrm{K}, 0,2.3721502018,0.0011485248,-0.0563911443$ C,0,5.1091325662,-0.0801173859,-1.172023262 C,0,5.0852474098,1.1213381134,-0.4150731689 C,0,5.0788441958,-1.1716573482,-0.2639302862 H,0,5.2006153354,-0.151755892,-2.252920828 C,0,5.0403030871,0.7723309343,0.9607278521 H,0,5.1537582624,2.1295536495,-0.8158016044 C,0,5.0362629647,-0.6447878343,1.0541623465 H,0,5.1420334932,-2.2241185415,-0.5287471319 H,0,5.0683142917,1.466993401,1.7964564276 H,0,5.0611449794,-1.2238021297,1.9738922874 C,0,-3.2643435674,0.000229487,-0.0053744473 Li,0,-5.802794471,-2.7271503956,0.0414923873 Li,0,-5.796702337,2.7320328454,0.0425775144

\section{$\mathbf{H F}=\mathbf{- 1 8 1 6 . 3 6 2 8 3 7 4}$}

di

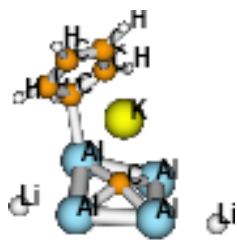

C,0,-2.1215531343,-1.2271613643,0.0220166305 C,0,-2.6903124062,-0.3611223918,1.0404472648 C,0,-3.2745721947,0.7364647395,0.4197659163 C,0,-3.1295586529,0.5783945998,-1.0072524122 C,0,-2.4521941922,-0.6103540694,-1.2486215669 H,0,-2.189694001,-2.3100788548,0.1254443569 H,0,-2.6800129171,-0.5605694216,2.1072464226 H,0,-3.7918342776,1.552379026,0.9189566086 
H,0,-3.5228390573,1.2549123211,-1.7627161059 H,0,-2.2301893075,-1.035875468,-2.2218011219 Al,0,2.9667573008,1.0142652431,-0.2212281211 Al,0,1.7824656235,-0.0126600327,2.0163190569 Al,0,0.1265628067,-1.6373899457,0.4371390056 Al,0,1.7363775197,-1.1073892338,-1.601117194 C,0,1.3767402309,-0.0820521793,0.0725892523 Li,0,3.9389977141,0.1118712599,-2.6081246567 Li,0,0.7640595029,-2.4419353301,2.8967446894 $\mathrm{K}, 0,-0.626527487,1.9234563262,-0.2121645517$

\section{$\mathrm{HF}=-\mathbf{1 8 1 6 . 3 6 5 0 3 6}$}

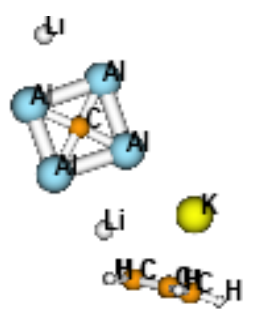

C, $0,-4.0472438647,0.9932784977,-0.531028484$ C,0,-4.5832909746,-0.3048895413,-0.7156833953 C,0,-4.048599687,-1.1529214718,0.2851185674 C,0,-3.1991811215,-0.3753232666,1.1122045132 C,0,-3.1981544718,0.9608666545,0.6040769462 H,0,-4.2985974878,1.8798870456,-1.1051639418 H,0,-5.3192656315,-0.588877261,-1.4636775093 $\mathrm{H}, 0,-4.3006665532,-2.1968839023,0.4447452307$ $\mathrm{H}, 0,-2.7854755656,-0.7094354654,2.0647143942$ H,0,-2.7839961388,1.842904644,1.0943354045 Al,0,3.9442642821,-0.494141811,-1.2394674813 Al,0,4.2618009572,0.5180024969,1.3814501155 Al,0,1.5757578655,0.6108116931,1.6826737772 Al,0,1.2213225827,-0.3768090954,-0.8880094147 K,0,-2.0393826194,-0.8044524488,-2.0272368663 C,0,2.7587597135,0.0442072924,0.2169495337 Li,0,-1.0779246341,0.3424944326,0.9922492067 Li,0,6.4139687412,-0.0485775391,-0.1734291044 HF =-1816.3969607

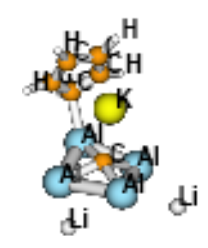

C,0,-2.1883620946,-1.3422270476,0.0077082747 C,0,-2.6345234463,-0.5769864201,1.1647244389 
C,0,-3.2532454378,0.5824183559,0.7243304218

C,0,-3.2547037282,0.5769596267,-0.7212775939

C,0,-2.6366608377,-0.5856383388,-1.1541721182

$\mathrm{H}, 0,-2.3113015044,-2.4267977535,0.0117569472$

H,0,-2.5158813099,-0.8881158928,2.1973152897

$\mathrm{H}, 0,-3.7039715012,1.3449128508,1.3556753517$

H,0,-3.7067239074,1.3346941926,-1.3574220645

H,0,-2.5199592478,-0.9044810451,-2.1846280772

Al,0,1.6576060645,-0.7989923862,1.831156785

Al,0,0.030566909,-1.6700609238,0.0066680664

Al,0,1.6536453237,-0.8135229567,-1.8280872165

Al,0,2.9028885403,0.9617774743,-0.0067356158

K,0,-0.7008628399,1.9454945376,-0.0077640258

C,0,1.2540332858,-0.0395298761,-0.0010024649

Li,0,3.8594130326,0.4992542881,-2.5862800188

Li,0,3.8651923321,0.5193450235,2.5742557002

HF $=-\mathbf{1 8 1 6 . 3 5 3 9 0 1 2}$

di

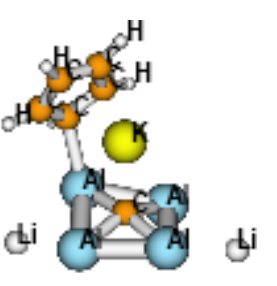

C,0,-2.693437556,0.9860504751,-0.4814091478

C,0,-3.2971645691,-0.2427878676,-0.7197079854

C,0,-3.1312347032,-1.0629171651,0.4559943636

C,0,-2.4217525876,-0.3329074486,1.4014723223

C,0,-2.091084405,0.9634206192,0.8405192137

$\mathrm{H}, 0,-2.6913220423,1.837381249,-1.154595751$

H,0,-3.8408936707,-0.5238160353,-1.6185088362

H,0,-3.5320646487,-2.0649682155,0.5911950126

$\mathrm{H}, 0,-2.1772853366,-0.654355684,2.408606767$

$\mathrm{H}, 0,-2.1341159349,1.8484026437,1.4753641135$

Al,0,2.9438427039,-0.9441695626,-0.5699481922

Al,0,1.7614765447,1.3148790695,-1.5526065768

Al,0,0.1613808008,1.5184139653,0.7421158893

Al,0,1.7799353604,-0.2369415863,1.8925894592

C,0,1.3772030113,0.092319598,-0.0341373439

Li,0,3.9624197937,-1.8421290261,1.7990636352

Li,0,0.7935173595,3.7353873027,-0.5940890675

$\mathrm{K}, 0,-0.6712402787,-1.5799922405,-1.0921753198$

HF $=-\mathbf{1 8 1 6 . 3 6 5 0 3 6 5}$ 


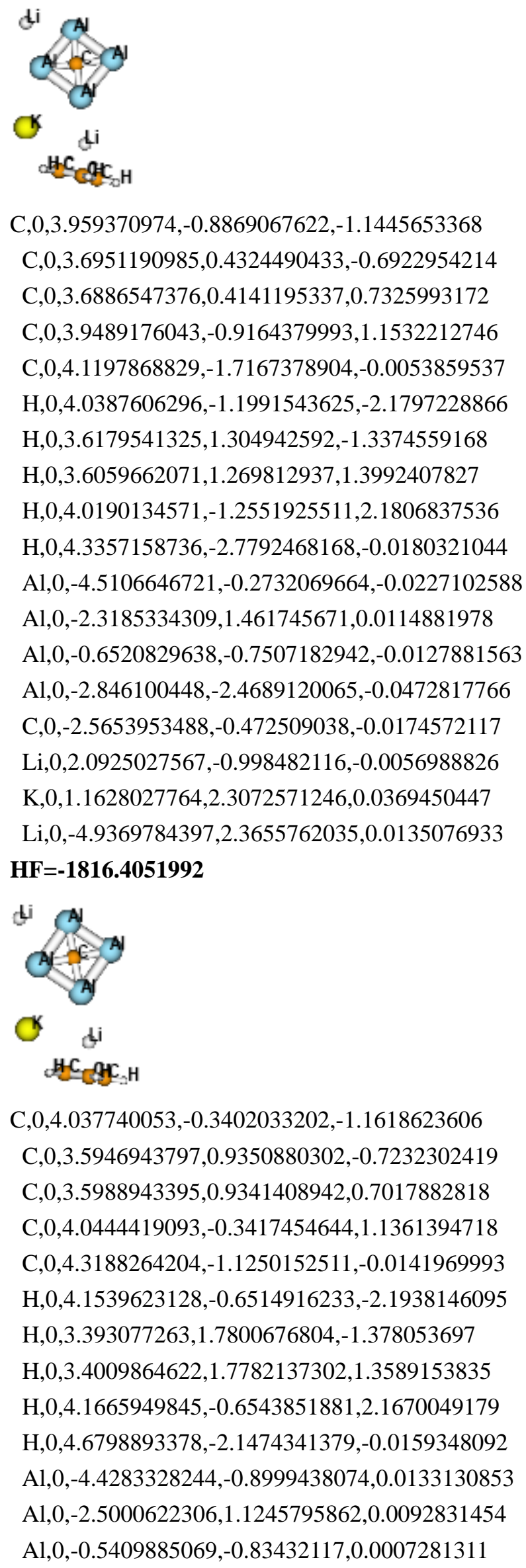

C,0,3.959370974,-0.8869067622,-1.1445653368

C,0,3.6951190985,0.4324490433,-0.6922954214

C,0,3.6886547376,0.4141195337,0.7325993172

C,0,3.9489176043,-0.9164379993,1.1532212746

C,0,4.1197868829,-1.7167378904,-0.0053859537

H,0,4.0387606296,-1.1991543625,-2.1797228866

H,0,3.6179541325,1.304942592,-1.3374559168

H,0,3.6059662071,1.269812937,1.3992407827

H,0,4.0190134571,-1.2551925511,2.1806837536

H,0,4.3357158736,-2.7792468168,-0.0180321044

$\mathrm{Al}, 0,-4.5106646721,-0.2732069664,-0.0227102588$

Al,0,-2.3185334309,1.461745671,0.0114881978

Al,0,-0.6520829638,-0.7507182942,-0.0127881563

$\mathrm{Al}, 0,-2.846100448,-2.4689120065,-0.0472817766$

C,0,-2.5653953488,-0.472509038,-0.0174572117

Li,0,2.0925027567,-0.998482116,-0.0056988826

K,0,1.1628027764,2.3072571246,0.0369450447

Li,0,-4.9369784397,2.3655762035,0.0135076933

HF $=-\mathbf{1 8 1 6} .4051992$

C,0,4.037740053,-0.3402033202,-1.1618623606

C,0,3.5946943797,0.9350880302,-0.7232302419

C,0,3.5988943395,0.9341408942,0.7017882818

C,0,4.0444419093,-0.3417454644,1.1361394718

C,0,4.3188264204,-1.1250152511,-0.0141969993

H,0,4.1539623128,-0.6514916233,-2.1938146095

H,0,3.393077263,1.7800676804,-1.378053697

H,0,3.4009864622,1.7782137302,1.3589153835

H,0,4.1665949845,-0.6543851881,2.1670049179

H,0,4.6798893378,-2.1474341379,-0.0159348092

Al,0,-4.4283328244,-0.8999438074,0.0133130853

Al,0,-2.5000622306,1.1245795862,0.0092831454

$\mathrm{Al}, 0,-0.5409885069,-0.83432117,0.0007281311$ 
Al,0,-2.4735172877,-2.8419752664,0.0047707509

C,0,-2.4743313896,-0.8254746985,0.0070232669

Li,0,2.2114053536,-0.696687469,-0.0077229832

Li,0,-5.21836631,1.6539125691,0.0187099178

K,0,0.829604699,2.446010613,-0.0005412088

$\mathrm{HF}=-\mathbf{1 8 1 6 . 4 0 5 1 9 9 1}$

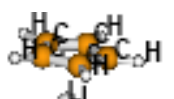

ti

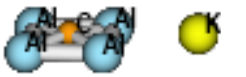

C,0,-2.4801326221,-1.1623481208,1.3116691403

C, $0,-2.0061052823,-0.7265078548,2.5773726045$

C,0,-1.9996884623,0.6948880527,2.579972838

C,0,-2.4698554482,1.1395415551,1.3159440531

C,0,-2.7680610014,-0.008673806,0.5328435501

H,0,-2.6136581282,-2.1929048659,1.0014869803

H,0,-1.7143401551,-1.3677728764,3.4020108093

$\mathrm{H}, 0,-1.702033428,1.3305653314,3.40682369$

H,0,-2.5947394123,2.1724420252,1.0099505563

H,0,-3.1808086757,-0.00524219,-0.4710586073

Al,0,2.1993493617,1.378325653,1.2429073107

Al,0,2.1918209065,-1.4458084667,1.1912632085

Al,0,0.9184639178,-1.4219849674,-1.2071465061

Al,0,0.9250766576,1.449884174,-1.1533678121

C,0,1.3353314114,-0.0117940436,0.131961622

Li,0,3.4866702562,-0.0722185049,3.1534224347

K,0,-0.8097240261,0.0685607991,-3.8218039807

Li,0,-0.6632823363,-0.0197116322,1.0495604794

HF $=-\mathbf{1 8 1 6 . 4 0 7 3 2 0 2}$

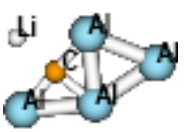

ck

HCeft $\mathrm{H}$

di

Al,0,4.7447418642,-0.1295595048,0.0231634832

Al,0,1.5240930809,-2.2488125644,-0.0047845391 Al,0,2.1354233256,0.4779217256,-0.0108944028 $\mathrm{Al}, 0,3.9886573784,2.6003760653,0.0010398237$ C,0,3.1522287352,-1.2113131853,0.0124280176

K,0,-1.3260763923,-0.1604777551,-0.0482088318 C,0,-4.2718877038,0.4821979499,-1.1714673756

C,0,-4.4256942204,-0.8057090736,-0.5821197663 
C,0,-4.1215733352,1.4322566982,-0.1206041191

H,0,-4.2679599023,0.7010066545,-2.2337144286

C,0,-4.3704222382,-0.6516794973,0.8328292918

H,0,-4.5558796445,-1.7403892574,-1.1163820969

C,0,-4.1825376449,0.731503873,1.1181788812

H,0,-3.9809845082,2.5006925534,-0.2422108867

H,0,-4.4512730808,-1.4486816379,1.5638008685

H,0,-4.0977575025,1.1734553317,2.1049523535

Li,0,-6.0158711964,0.4645449453,0.0589099335

Li,0,4.2694448957,-2.8310720346,0.0055019568

HF $=-\mathbf{1 8 1 6 . 3 6 9 1 8 5 4}$ 


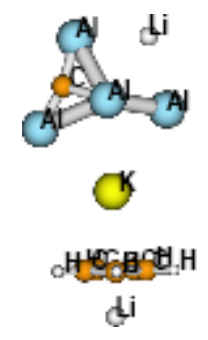

Al,0,-4.8915090972,-0.5847123088,-0.4101246269 Al,0,-1.6592670759,-2.2896562126,-0.1220136518 Al,0,-2.5694458201,0.390164266,0.9328387391 Al,0,-1.8511810302,2.5394953242,-0.2568290285 C,0,-3.2161363985,-1.2517139219,0.1836846903 $\mathrm{K}, 0,1.0170381765,0.1652285205,-0.1399192902$ C,0,3.9236514416,-0.0067244406,1.2083923439 C,0,3.8529840783,-1.1906879354,0.4193421619 C,0,4.082009591,1.1015298773,0.3270085769 $\mathrm{H}, 0,3.8602593865,0.0427982639,2.2899269587$ C,0,3.9677806432,-0.814394579,-0.9496486464 H,0,3.7203829887,-2.1999190428,0.7929207005 C,0,4.1091297709,0.6023150557,-1.006902587 H,0,4.159533724,2.1428959949,0.6193412758 H,0,3.9425412059,-1.4885807673,-1.7986342555 H,0,4.2133531498,1.1971938485,-1.9077011647 Li,0,5.7292980418,-0.2339389365,0.1008669458 Li,0,-4.6986551296,2.1691194642,-0.2005252306 HF =-1816.3528595

di

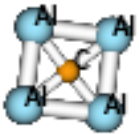

\section{H-gage-OH}

di

Al,0,-4.7607467891,1.0434290907,0.0045017456 Al,0,-1.9545234992,1.3458472238,-0.004624462 Al,0,-1.7405494707,-1.4444096584,-0.0024745626 Al,0,-4.4923085211,-1.6886953256,0.0066175049 K,0,1.4069503372,-0.0091836602,-0.0190941148 C,0,4.3826659137,1.1981333096,-0.2059402386 C,0,4.3807021256,0.5768794829,1.0761408709 C,0,4.3876848871,0.1702975468,-1.1925908749 H,0,4.3787100247,2.265652183,-0.3971242209 C,0,4.3840474552,-0.8344716578,0.8818005816 H,0,4.375185954,1.088745218,2.0323039695 C,0,4.3884541785,-1.085694683,-0.5201694205 
H,0,4.3899812901,0.3180545717,-2.2670733609

H,0,4.3794755156,-1.5861312824,1.663476965

H,0,4.3870528676,-2.062005796,-0.992237129

C,0,-3.2065655333,-0.1379113594,0.0013401877

Li,0,6.1416078965,0.0085920536,0.0115420054

Li,0,-3.6811840896,3.4902584523,-0.0023112105

HF $=-\mathbf{1 8 1 6} .3934635$

لو

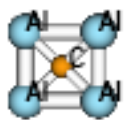

ck

CHCACSCH

di

Al,0,1.4000418084,-4.5069960963,-0.010291537

Al,0,1.4404031977,-1.7896471433,-0.0113633114

Al,0,-1.396295054,-1.7494943938,0.0093045087

Al,0,-1.4319380633,-4.4674889046,0.0102949289

K,0,0.0699255286,1.4619374512,-0.0013721527

C,0,1.1801547786,4.4861228458,0.004822818

C,0,0.3465481052,4.4585715564,-1.150025832

C,0,0.3397594694,4.4551542593,1.1546780611

$\mathrm{H}, 0,2.2641019922,4.520312458,0.0080565514$

C,0,-1.0088127309,4.4101665645,-0.7138288617

H,0,0.6849820291,4.4697725416,-2.180384593

C,0,-1.0130216882,4.4080026952,0.7104052643

$\mathrm{H}, 0,0.6721176728,4.4634812105,2.1870431837$

H,0,-1.8840120764,4.3755740535,-1.3532888434

H,0,-1.8919546802,4.3714854212,1.3446135974

C,0,0.0027727947,-3.1359137131,-0.0005756231

Li,0,-0.0907895582,6.1987877164,0.0035947902

Li,0,-0.0482034107,-6.7964268872,0.0010373382

HF =-1816.3987799 


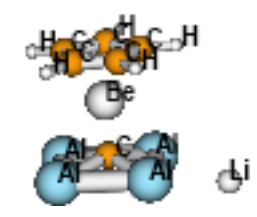

C,0,-2.1449284542,1.4004932768,-0.277076381

C,0,-2.2639513746,0.3314133869,-1.2098562115

C, $0,-2.4078307555,-0.8786855421,-0.4748824182$

C,0,-2.3745693599,-0.5587189819,0.911222462

C,0,-2.2116342184,0.8500743475,1.033934372

H,0,-1.9791591435,2.4418029986,-0.5216691559

$\mathrm{H}, 0,-2.2007989145,0.4160218745,-2.287075532$

H,0,-2.4718448731,-1.8742208685,-0.8948854335

H,0,-2.4147160847,-1.2673196141,1.7284522374

H,0,-2.1078789998,1.3980813102,1.9616606865

Al,0,1.1429519521,-1.6931964437,-1.396857422

Al,0,1.5529590255,1.0441114616,-1.4475959148

Al,0,1.5464935946,1.0477494223,1.4494945604

Al,0,1.1357435698,-1.6912134479,1.4025052966

Be,0,-0.7769965701,0.0596111368,0.0017091235

C,0,0.922036792,-0.1757705236,0.0012965917

Li,0,2.4172408913,3.2124970237,0.0002484839

\section{HF =-1223.6910435}

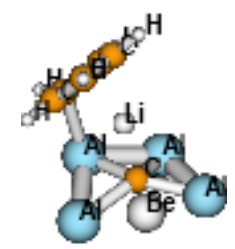

C,0,-2.6606746419,-1.0644479617,1.088782731

C,0,-1.6160612774,-0.6995115357,1.9388682406

C,0,-1.3209820014,0.705278719,1.7328487273

C,0,-2.2855820007,1.1800354496,0.7591956332

C,0,-3.0760926041,0.1034739807,0.3544665748

H,0,-3.0942604024,-2.0548332834,1.0003710388

H,0,-1.1110251788,-1.3478659279,2.645602032

H,0,-0.9685143893,1.3409501277,2.5452852447

$\mathrm{H}, 0,-2.376938155,2.2043001768,0.4162195894$

H,0,-3.8747206147,0.139383815,-0.3786736693

Al,0,0.8521293856,0.9860887929,0.8837744109

Al,0,2.0060898963,-1.2410765793,0.5359555607

Al,0,1.7470758035,-1.8035092379,-1.9600703539

Al,0,0.9403754059,2.0766908408,-1.412626058

C,0,0.912752883,-0.1433668783,-0.8704166192

Li,0,-1.0811500743,-0.4852907489,-0.2963335653 
Be,0,0.7137576077,0.1121617701,-2.4934278852

HF=-1223.645983

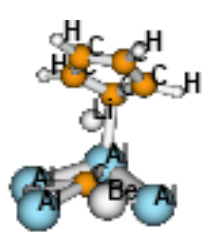

C,0,2.2611920961,1.1697035377,0.8426132519

C,0,1.2545771573,0.710009236,1.7800987775

C,0,1.530440069,-0.6950053027,2.0093829254

C,0,2.606012458,-1.0745431491,1.2054132223

C,0,3.0594773301,0.084142534,0.4790210132

$\mathrm{H}, 0,2.374282112,2.1904634521,0.4957098971$

H,0,0.8728352653,1.3550083409,2.5716416321

H,0,0.9922594126,-1.3338280015,2.7001452567

H,0,3.0352361354,-2.0687716122,1.1425093592

H,0,3.8875285309,0.1083145839,-0.2212075292

$\mathrm{Al}, 0,-2.03519148,-1.223259915,0.4627779609$

Al,0,-0.8802673864,0.9986833316,0.840201266

Al,0,-0.8686079164,2.0726502133,-1.4657302674

Al,0,-1.6774365932,-1.8061635858,-2.0163619648

C,0,-0.8770030219,-0.1437466435,-0.9058575672

Li,0,1.0884755141,-0.4971059754,-0.2469402521

Be,0,-0.6090501604,0.0979848291,-2.5208846324

HF $=-\mathbf{1 2 2 3 . 6 4 5 9 8 3}$

di
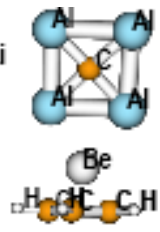

Al,0,1.264475021,-3.2912883679,-0.0087529146 Al,0,1.2078561835,-0.5312409336,-0.007211446 $\mathrm{Al}, 0,-1.515395786,-0.5281104396,0.0085130503$ Al,0,-1.5886112888,-3.3102389473,0.0076078148 Be,0,-0.0282090843,1.6373849515,0.0009557902 C,0,1.1618183964,3.194268625,0.0040733074 C,0,0.3293847669,3.1664905674,-1.1466616323 C,0,0.3247729541,3.1649299658,1.151561115 $\mathrm{H}, 0,2.2424712551,3.1461610591,0.0062066384$ C,0,-1.0252429148,3.1443960801,-0.7097056354 H,0,0.6660057532,3.1203657164,-2.1744106159 C,0,-1.0280663699,3.1436751556,0.7092541581 $\mathrm{H}, 0,0.6573294089,3.1177013551,2.1805838867$ H,0,-1.8971991637,3.06207238,-1.3447399135 
H,0,-1.9025101458,3.0602636842,1.3406889002

C,0,-0.1278479319,-1.9362113969,-0.0002296039

Li,0,3.5832040457,-1.9099916779,-0.0213122895

HF $=-\mathbf{1 2 2 3 . 6 7 7 3 5 3 3}$

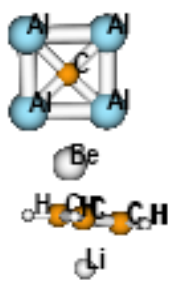

Al,0,1.3963916086,-3.7519803325,0.0020556205 Al,0,1.6136496625,-0.9657254183,0.0017141948 Al,0,-1.1550376338,-0.7315852997,-0.0030982874 Al,0,-1.4392044572,-3.482384684,-0.0028411254 Be,0,0.3746610353,1.0673570901,-0.0002170584 C,0,0.9580578203,2.8744839026,0.0027414337 C,0,0.1198072584,3.0609864496,-1.158515163 C,0,0.1149587648,3.0579774701,1.1609945283 $\mathrm{H}, 0,2.0362457328,2.7618003445,0.004673667$ C,0,-1.1847057623,3.3856999176,-0.7100235958 $\mathrm{H}, 0,0.4419401405,2.9730728027,-2.1892581521$ C,0,-1.187666916,3.3838584718,0.7078586629 $\mathrm{H}, 0,0.4327616195,2.9675026596,2.1928557645$ H,0,-2.0529561564,3.5191858027,-1.3433512337 H,0,-2.058540507,3.51575463,1.3379055284 C,0,0.1202999182,-2.1873525421,-0.0007148332 Li,0,0.2173363961,4.8837059771,0.0040670769 HF $=-\mathbf{1 2 2 3 . 6 3 5 5 9 5 4}$

di
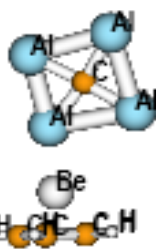

Al,0,1.4693783056,-2.7576259299,-0.0035871921 Al,0,0.7264391082,-0.3160957698,-0.0014807459 Al,0,-2.1016876949,-0.9715481567,0.0040443998 Al,0,-1.1482723112,-3.6873164326,0.0014056274 Be, $0,0.4217755474,1.9642211379,-0.0003572197$ C,0,1.5308031159,3.5359505744,0.0020321026 C,0,0.6986592806,3.4740565338,-1.1496708511 C,0,0.6950702235,3.4729718221,1.1511161518 H,0,2.6129992069,3.5486439607,0.0037157255 C,0,-0.6530150697,3.3793949229,-0.7120209348 
H,0,1.0373716681,3.4400877815,-2.1770937196

C,0,-0.6552335335,3.3787743191,0.7091960952

H,0,1.0305845634,3.4380914649,2.1795575617

H,0,-1.5204024248,3.2503295522,-1.3457344316

$\mathrm{H}, 0,-1.5245724743,3.2490093668,1.3400778301$

C,0,-0.3601221288,-1.911862755,0.0002988112

Li,0,0.9479332145,-5.4117124749,-0.0032564992

HF $=-\mathbf{1 2 2 3 . 6 8 5 1 4 4}$ 


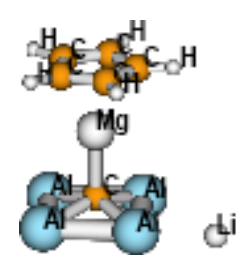

C,0,-2.8013738228,0.3389874016,-1.1961930269

C,0,-2.8513490997,-0.9863062521,-0.6731735714

C,0,-2.8601097005,-0.8984109013,0.7499299682

C,0,-2.8154554349,0.4811998435,1.1065975992

C,0,-2.7790094118,1.2458180287,-0.0962490091

H,0,-2.8077411636,0.6096608504,-2.2457153187

H,0,-2.9020083777,-1.899166843,-1.2552598976

H,0,-2.9181048835,-1.7329567907,1.4390509798

$\mathrm{H}, 0,-2.8346230418,0.8787495011,2.1147660908$

H,0,-2.7692420532,2.3276783115,-0.1631550972

Al,0,1.7365131021,-1.5441289575,1.4434917515

Al,0,1.7576962833,-1.6156271147,-1.3362856828

Al,0,1.8551998767,1.1406541478,-1.4613858672

Al,0,1.8329930481,1.2150106191,1.4282180253

Mg,0,-0.8164483779,-0.0390020514,-0.005262167

C,0,1.2740751943,-0.0935414046,0.0117618855

$\mathrm{Li}, 0,2.5524012249,3.4035887534,-0.068360258$

$H F=-1409.0130179$

C,0,-2.4164973209,1.0362610933,-0.2082564602

C,0,-2.3724220071,0.774015634,-1.6071596508

C,0,-2.5914548708,-0.6124964485,-1.8017914398

C,0,-2.7722271942,-1.2158729789,-0.525295712

C, $0,-2.6697076725,-0.1984324535,0.4579128704$

$\mathrm{H}, 0,-2.3822628798,2.0165796292,0.2539657897$

H,0,-2.221374135,1.5116830352,-2.3871342957

H,0,-2.6274218044,-1.123649158,-2.7573210215

H,0,-2.9769878592,-2.2642795813,-0.3392196715

H,0,-2.8181717299,-0.3322361258,1.5243408545

Al,0,2.3126540106,-1.5741534854,-1.0659359952

Al,0,2.0585487653,1.0253841029,-1.6377872652

Al,0,0.7502291149,1.6748258225,0.6170493758

Al,0,1.1916850219,-1.4070862515,1.3771498472

C,0,1.2619526575,-0.0949662173,-0.0585789073

Li,0,-0.8106392362,-0.3660259731,-0.6565089046 
Mg,0,0.229146225,0.5676929912,3.113143612

HF=-1409.005966

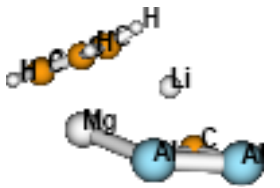

C,0,-2.7422769727,-0.751266823,1.1289618461

C, $0,-3.5034303702,0.372429962,0.7358888135$

C,0,-3.505363375,0.4262575899,-0.7002833137

C,0,-2.7453352009,-0.6647720222,-1.1784261849

C,0,-2.2604719737,-1.3905908392,-0.0509701046

H,0,-2.5748569971,-1.0774562136,2.1486483889

$\mathrm{H}, 0,-4.0473203895,1.0352346447,1.4001388157$

H,0,-4.0511233137,1.1367661763,-1.311582722

H,0,-2.5805856584,-0.9136745902,-2.220098644

H,0,-1.8403544011,-2.3984165825,-0.0894117391

Al,0,0.9513026404,0.8640896912,-1.4840944325

Al,0,0.9530832144,0.7536675671,1.5422565289

Al,0,3.2579110901,-0.6683103161,1.3432439594

Al,0,3.2561928843,-0.5684245033,-1.3922216427

C,0,1.7873346075,-0.1876726868,-0.007793073

Li,0,0.0781673354,-1.4008516735,-0.0511805629

Mg,0,-1.396947443,1.2200415662,0.0451818599

$H F=-1409.0120485$

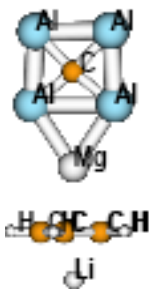

Al,0,1.3897343051,-3.9716417444,-0.0126730294

Al,0,1.5114602185,-1.2530511464,-0.0132093554

Al,0,-1.4917407074,-1.2560854869,0.0125971735

Al,0,-1.3644052074,-3.9745048588,0.0108838371

Mg,0,0.0075521299,1.0845179084,0.0000369429

C,0,1.1956444233,3.4953089615,0.0047597007

C,0,0.36026423,3.4875549348,-1.1520639234

C,0,0.3527210622,3.486597773,1.1561929183

$\mathrm{H}, 0,2.2785259039,3.4728323906,0.0082546543$

C,0,-0.9996500622,3.4821760909,-0.7151290954

$\mathrm{H}, 0,0.699640385,3.4750862929,-2.1811340546$

C,0,-1.0043066586,3.481710292,0.7104855845

H,0,0.6853776139,3.4735905918,2.1874574313 
H,0,-1.8746749087,3.4533263097,-1.3531812248

H,0,-1.8834352443,3.4522306223,1.3428283454

C,0,0.0110900362,-2.4733861048,-0.0005539637

Li,0,-0.0254231362,5.2725430934,0.0014673569

\section{$H F=-1409.0065567$}

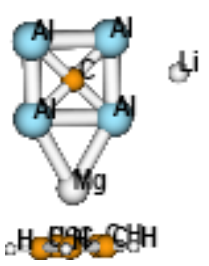

Al,0,1.8735242628,-3.5466202679,-0.0892156615 $\mathrm{Al}, 0,1.5638449788,-0.822244661,0.073208038$ Al,0,-1.2213431807,-1.0910524276,-0.1703760852 Al,0,-0.8838659757,-3.8357581955,-0.331647977 C,0,0.9416917184,3.6801624553,0.4070347315 C, $0,0.2912198575,3.6784171982,-0.8591360591$ C,0,-0.0521728452,3.51143428,1.4127985009 H,0,2.005583389,3.7977300094,0.5762813598 C,0,-1.1078703396,3.5167667112,-0.6351527913 H,0,0.7720579368,3.8089145448,-1.8219300034 C,0,-1.3197145478,3.41464884,0.7672556484 H,0,0.1213476367,3.495203138,2.4828038028 H,0,-1.8766577729,3.4942375244,-1.3989749964 H,0,-2.2781774969,3.2993632618,1.2601159563 C,0,0.2938901056,-2.2926038077,-0.1305488936 Li,0,-3.3748074216,-2.8111182206,-0.4633721254 Mg,0,-0.0183396857,1.5273945247,0.1043937791 $H F=-1409.0224861$

bi

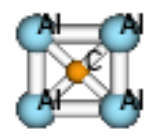

Cag

\section{CH-SFE-CH}

Al,0,1.4201089332,-3.5886369428,-0.0177824754 Al,0,1.420196406,-0.8774528992,-0.0166528649 Al,0,-1.4052790846,-0.889568557,0.0164399484 Al,0,-1.3854050709,-3.5981225465,0.0152223908 Mg,0,-0.0064947565,1.6088464525,0.000704068 C,0,1.1911369398,3.6739604512,0.0059149202 C,0,0.3593690562,3.6643138877,-1.1488789318 C,0,0.3500093821,3.6635917763,1.1540670386 $\mathrm{H}, 0,2.2747771089,3.6899278929,0.0103014861$ C,0,-0.9986479999,3.6573189106,-0.7135621576 
H,0,0.6987037011,3.690074602,-2.1783007488

C,0,-1.0043834741,3.6570469697,0.7079206077

H,0,0.681079896,3.6893226746,2.1861879849

H,0,-1.8733239858,3.6654375338,-1.3537777269

H,0,-1.8841359973,3.6646096566,1.3411304624

C,0,0.0130447479,-2.2288403097,-0.0006609313

Li,0,0.0241963516,-5.9435758715,-0.0022481781

HF $=-\mathbf{1 4 0 9 . 0 2 8 4 2 4 3}$ 


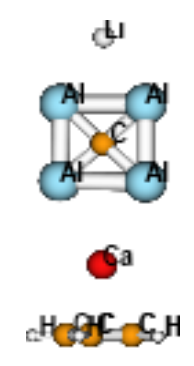

Al,0,1.4251369259,-4.0213082008,-0.01316018 Al,0,1.4304072336,-1.3131180589,-0.0103755227 Al,0,-1.4146710543,-1.3263579748,0.0116871393 Al,0,-1.3848833637,-4.0339074595,0.0085873819 Ca,0,-0.0064747633,1.608977275,0.0019216014 C,0,1.1881998806,4.0537511163,0.0043668058 C,0,0.3556487254,4.0476355379,-1.1489373318 C,0,0.3485757359,4.0489910178,1.1525745994 $\mathrm{H}, 0,2.271813654,4.1121593266,0.0076492854$ C,0,-0.9991725891,4.040017718,-0.7132930366 $\mathrm{H}, 0,0.6939780169,4.107680717,-2.1785557312$ C,0,-1.0035533935,4.0408587824,0.7086544613 $\mathrm{H}, 0,0.6805522172,4.1104846785,2.1841795687$ H,0,-1.87488688524,4.0883944543,-1.3525304302 $\mathrm{H}, 0,-1.8831561143,4.0899492494,1.3424691894$ C,0,0.0140450268,-2.657492138,-0.0006124957 Li,0,0.0302891289,-6.3665980367,-0.0052555217

\section{HF =-1886.5061434}

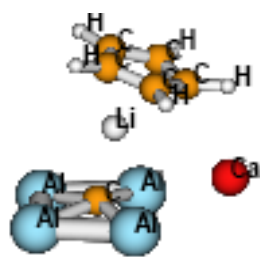

C,0,-2.5044974553,-1.1649887275,1.131430178 C,0,-2.0589196047,-0.7310199633,2.3973506067 C,0,-2.0562639082,0.6944175786,2.4068257742 C,0,-2.5004533352,1.1468149556,1.1468742864 C,0,-2.7701848492,-0.0032877829,0.3388800713 $\mathrm{H}, 0,-2.6539455976,-2.1950548363,0.8279800148$ H,0,-1.7683699625,-1.3735605694,3.220934379 $\mathrm{H}, 0,-1.763359923,1.3249099927,3.238835811$ $\mathrm{H}, 0,-2.6465942391,2.1814377271,0.8575930523$ H, $0,-3.4206805337,0.0033086403,-0.5390614063$ Al,0,2.6454168918,1.3128221522,1.0959347095 Al,0,2.6418736703,-1.3475829441,1.0662550749 $\mathrm{Al}, 0,0.9677609639,-1.6117915847,-0.9878734341$ 
Al,0,0.971727692,1.6274584994,-0.9513948665

C,0,1.4099982466,-0.0044233693,0.0646563278

Li,0,-0.550970703,-0.0131140682,1.0352066992

Ca,0,-1.374924083,0.0251130599,-2.2500373483

HF $=-\mathbf{1 8 8 6 . 4 7 7 1 6 3 9}$

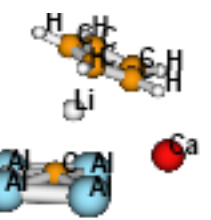

C,0,-2.8144851018,-1.0686825685,0.8305471399 C,0,-2.0187834613,-0.6410805138,1.9267873975 C, $0,-1.9621030527,0.7867328225,1.8947799581$ C,0,-2.7233677122,1.2265568627,0.7790933452 C, $0,-3.2570959438,0.0832797845,0.1340712457$ H,0,-3.0568052733,-2.0971891329,0.5901782666 H,0,-1.6773855923,-1.3003659976,2.7238942552 H,0,-1.5681181396,1.4518977614,2.6621911925 H,0,-2.8838611289,2.2592414982,0.4925163987 H,0,-3.88943574699,0.0886363133,-0.7467655834 Al,0,1.4474591263,1.5084753666,-0.1287236362 Al,0,1.3192615025,-1.7205229736,-0.0563813492 Al,0,0.748852514,-1.4704585073,-2.649051408 Al,0,0.8544282377,1.188716044,-2.7086271741 C,0,0.8337991838,-0.1063823488,-1.0824261606 Li,0,-1.1332606836,-0.0071225181,-0.1377323463 Ca,0,0.8784764625,-0.027171854,2.5322603484

\section{HF $=-\mathbf{1 8 8 6 . 4 7 7 1 9 0 4}$}

di
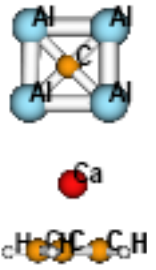

Al,0,1.2907768356,-4.1814423458,-0.0182943867 Al,0,1.3773042052,-1.4015405976,-0.016216622 Al,0,-1.4403288448,-1.3978685965,0.020266627 Al,0,-1.4668327137,-4.1525099576,0.0174478862 Ca,0,-0.1508719752,1.5376829208,0.0047416464 C,0,1.0740198635,3.9679917622,0.0071605573 C,0,0.2446804419,3.9688960535,-1.1485121272 C,0,0.2312879549,3.9734600499,1.1531510952 H,0,2.1581142123,4.016498435,0.0133717866 C,0,-1.1112880116,3.9756437625,-0.7165540365 
H,0,0.5861454514,4.0248117499,--2.1773155489

C,0,-1.1195866312,3.9784464188,0.7054472025

$\mathrm{H}, 0,0.5607489377,4.0337820431,2.1856219418$

H,0,-1.9847031129,4.032985547,-1.3581374428

$\mathrm{H}, 0,-2.0003628064,4.0382340086,1.3366464903$

C,0,-0.0268971014,-2.735300737,0.0006794409

Li,0,3.6840814846,-2.9803648635,-0.0482995024

HF $=-\mathbf{1 8 8 6 . 4 9 9 1 8 4 5}$ 


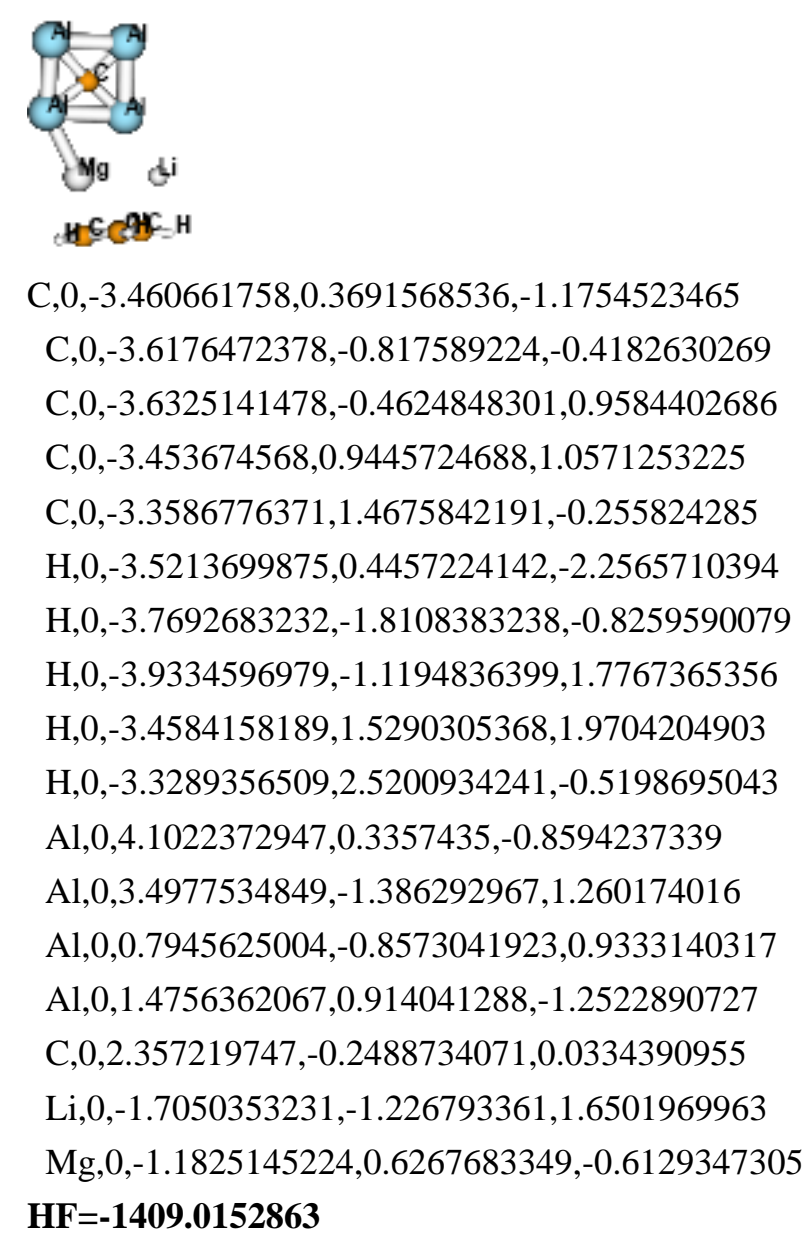




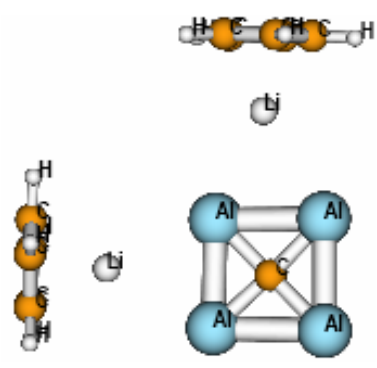

C,0,4.3555796619,0.5470927823,-0.5710644094 C,0,4.3447201789,1.8519418912,-1.1326096117 C,0,4.3278630098,0.6776424043,0.8410770459 H,0,4.3483297684,-0.3869645081,-1.1240130889 C,0,4.3128439337,2.7887290709,-0.0664708601 H,0,4.3349245672,2.0908777589,-2.1918995134 C,0,4.3005162541,2.0634308697,1.1533606947 H,0,4.2965568082,-0.1394786639,1.5551136362 H,0,4.2562434158,3.8678484412,-0.1668136819 H,0,4.2490694422,2.4925897132,2.1494965259 Al,0,-0.3396591297,0.3395478419,-0.0136432646 Al,0,-0.3128941792,3.08542355,-0.0542829229 Al,0,-3.142380689,3.1028691704,-0.0913777183 Al,0,-3.081790247,0.2780942231,-0.0488940218 $\mathrm{Li}, 0,2.4048142014,1.560044846,0.0090789793$ C,0,-1.737683144,1.7216517848,-0.0523090996 Li,0,-1.5738605555,-2.4167809565,0.0103037658 C,0,-0.3363584257,-4.3235274006,-0.0210593642 C,0,-1.1119176513,-4.316268222,1.167434714 C,0,-1.2277422085,-4.3515254432,-1.1254071157 H,0,0.7461251718,-4.2693286678,-0.0761108232 C,0,-2.4834759982,-4.3427413418,0.7976057857 H,0,-0.7245106328,-4.2691553451,2.1807383185 C,0,-2.5548978522,-4.3648869407,-0.6190457618 H,0,-0.9444152478,-4.3372975171,-2.1736064621 H,0,-3.3295633642,-4.3125361021,1.4771776385 H,0,-3.4648922342,-4.3527357165,-1.2107298331

\section{HF $=-\mathbf{1 4 1 0 . 0 7 9 5 8 3 1}$}

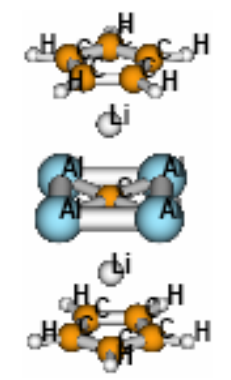

C,0,-4.18869804,-0.3078073704,-1.181345453

C,0,-4.1857644207,-1.2205709797,-0.0762621021 
C,0,-4.1915030113,-0.4517766473,1.133384667

C,0,-4.1979270084,0.9361965918,0.77593931

C,0,-4.1958658553,1.0251754996,-0.6546155143

C,0,4.1934961621,0.3031697586,1.1800179121

C,0,4.187335724,-1.0305282178,0.6551220301

C,0,4.1880276651,-0.9435717125,-0.7755087305

C,0,4.19421302,0.4438627765,-1.1349232467

C,0,4.1975530571,1.2144304526,0.0736371289

H,0,-4.1575764041,-0.5779420096,-2.2313632375

H,0,-4.1521369167,-2.3026153453,-0.1435529875

H,0,-4.1630507445,-0.850021802,2.1418986664

H,0,-4.174646368,1.7723216068,1.4665646307

H,0,-4.1705035213,1.9404379522,-1.2361488198

H,0,4.1667332538,0.5749339434,2.2297517055

H,0,4.1538505229,-1.9446716917,1.2379683899

H,0,4.1553764419,-1.7803976611,-1.4648686452

H,0,4.1682520122,0.8408567243,-2.1440008309

H,0,4.1749855032,2.2968876649,0.139421864

Al,0,0.0023548622,-1.5351749738,1.3855311904

Al,0,0.0010270019,-1.3822953414,-1.5374798613

Al,0,-0.0027344939,1.5413264073,-1.3851343201

Al,0,-0.0014066645,1.3885411243,1.5388014955

Li,0,-2.3042288122,0.0077977539,0.0023019835

Li,0,2.3042237425,0.0043216103,-0.0002415108

C,0,0.0005664164,0.0035346339,0.0005218798

\section{HF $=-448.2134547 \quad$ (B3LYP/LanL2DZ)}

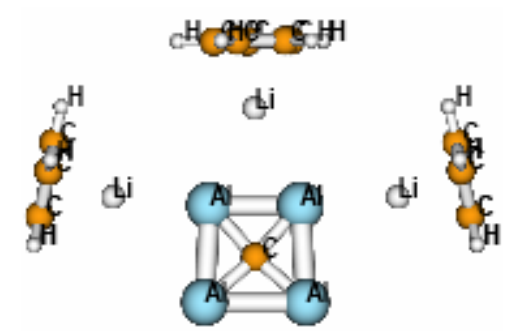

C,0,1.1704217743,4.0277127149,-0.509254589

C,0,-0.0726696028,4.074590366,-1.195379888

C,0,0.9014800231,4.020986038,0.8839159208

H,0,2.1524715115,3.9706750795,-0.967734129

C,0,-1.1097383387,4.0987020882,-0.2255001215

$\mathrm{H}, 0,-0.2073250896,4.0716011449,-2.2728413627$

C,0,-0.5079007925,4.0643784497,1.0593871404

H,0,1.6430268115,3.9612481451,1.6743381693

H,0,-2.1755377611,4.1037755701,-0.4299000389

H,0,-1.0347453104,4.0482969409,2.0085461349

$\mathrm{Al}, 0,1.3304641543,-0.6036669316,-0.0225719324$ 
Al,0,-1.3346126124,-0.5370223587,-0.0176969236

Al,0,-1.5253217068,-3.3051753314,-0.0056727605

Al,0,1.3656578169,-3.3949822134,-0.01066419

Li,0,-4.1651230383,-0.7051187551,0.0009306393

Li,0,0.0157016467,2.1709330132,-0.0180906547

C,0,-5.8273296597,0.8124061933,0.0081343749

C,0,-5.9785399093,-0.0197456146,-1.1319550211

C,0,-5.9534233245,-0.0016002419,1.1642440536

H,0,-5.6131591427,1.8764678077,-0.0024059255

C,0,-6.1998757891,-1.3485886323,-0.6803395448

H,0,-5.9121098946,0.2997394353,-2.1675003872

C,0,-6.1844054287,-1.337353351,0.7386760561

H,0,-5.8642003611,0.3343326688,2.1928287725

H,0,-6.3271805677,-2.2247465705,-1.3082815662

H,0,-6.2979281522,-2.2033922606,1.3830861854

C,0,-0.0369430609,-2.0143519009,-0.0152127388

Li,0,4.1507880139,-0.7599043354,-0.0113332778

C,0,5.8453342718,0.7193586682,-0.0038055766

C,0,5.9555931865,-0.1007699303,1.149652843

C,0,5.9747016543,-0.1125250019,-1.1468624376

H,0,5.6565967792,1.7882243802,-0.0105784652

C,0,6.1549769939,-1.4399907435,0.7195626005

H,0,5.8767875051,0.2341163017,2.1794174616

C,0,6.1667306805,-1.4473000147,-0.6997498293

H,0,5.9135222914,0.211736557,-2.181233061

H,0,6.2511095666,-2.3101926287,1.36119902

H,0,6.2736564012,-2.3240580065,-1.3306649005

HF $=-\mathbf{1 6 1 1 . 2 1 3 8 9 1 1}$

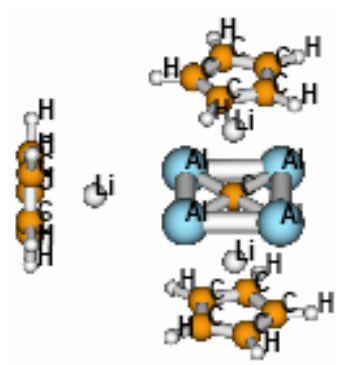

C,0,-0.729400309,4.7413642002,-1.0859424838

C,0,-1.3517021799,4.7273941592,0.1910192237

C,0,0.6748659853,4.7829029039,-0.8883544646

$\mathrm{H}, 0,-1.2367485714,4.6880998277,-2.0438041234$

C,0,-0.3310485674,4.7628364356,1.1773902798

H,0,-2.419355682,4.6723837919,0.3798006185

C,0,0.9214285369,4.7950720258,0.510863912

H,0,1.4285975409,4.7702032782,-1.6691235607

H,0,-0.4804513806,4.7266422362,2.2514947729 
H,0,1.8969498001,4.7982538376,0.9870631092

Al,0,-0.0153730366,0.1296998574,-1.4053544778 Al,0,-0.0174654099,0.133742773,1.4169388757 Al,0,0.1062688182,-2.7074903268,1.4628037269 Al,0,0.1066273436,-2.710832682,-1.4450296736 Li,0,-0.1065386599,2.8476694817,-0.0109662827 C,0,0.0451587741,-1.3063564794,0.0077724824 Li,0,-2.2791035896,-1.4193663993,0.0059137828 Li,0,2.3690508977,-1.2182301028,0.0044232138 C,0,-4.0671311989,-2.7006661705,0.0158491826 C,0,-4.1033714244,-1.8546804465,1.156644912 C,0,-4.0993095392,-1.8778020349,-1.1419386505 H,0,-3.9900876529,-3.7826861654,0.0270041331 C,0,-4.1581395487,-0.5091409185,0.703951649 $\mathrm{H}, 0,-4.0609800766,-2.1774856697,2.1914460294$ C,0,-4.1557241288,-0.5234327244,-0.716411241 H,0,-4.0528126049,-2.2211219803,-2.1699368974 H,0,-4.1677814167,0.3753419355,1.331669245 H,0,-4.1628455629,0.3482664369,-1.3618033093 C,0,4.1584875096,0.0628581755,-0.0458426115 C,0,4.1903555135,-0.7243660254,1.1364067124 C,0,4.1931204397,-0.817602163,-1.1602537707 H,0,4.0868099273,1.1442973483,-0.0900176293 C,0,4.2446630664,-2.0912357293,0.7526191985 H,0,4.1459293667,-0.3490431298,2.1532001911 C,0,4.2462411463,-2.1488067311,-0.6669745457 H,0,4.1516014608,-0.5257713546,-2.2041950692 H,0,4.2478818834,-2.9425737809,1.4249700344 H,0,4.2513522739,-3.051611495,-1.2683382591

\section{HF =-1611.2036867}

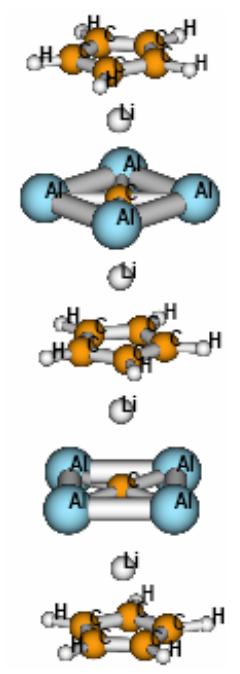

C,0,-8.4773952109,-0.7450590149,0.956649353 
C,0,-8.489863645,0.6878286353,0.9993084658

C,0,-8.4900387692,1.1711274695,-0.3501407872

C,0,-8.4776342214,0.0368070465,-1.2268370018

C,0,-8.4699681288,-1.1473417032,-0.4191005922

C, $0,-0.0052342133,-0.3610296841,-1.1594600931$

C,0,0.0020339604,0.9984074464,-0.6905116319

C,0,0.0077023214,0.9724699744,0.7471528392

C,0,0.0039821921,-0.4027546779,1.1669607624

C,0,-0.0041612473,-1.2270236947,-0.0113037279

H,0,-8.4439115525,-1.4078146128,1.8145039374

H,0,-8.4683783158,1.2988890959,1.8951645635

H,0,-8.4680362256,2.2120482281,-0.6542084619

H,0,-8.4436018631,0.0689550225,-2.310418411

H,0,-8.4289247444,-2.167716236,-0.7842762017

H,0,-0.0107991489,-0.6767530515,-2.1942074971

H,0,0.0032515036,1.8849501252,-1.310540248

$\mathrm{H}, 0,0.0141740356,1.8361196562,1.3986926367$

H,0,0.0073685858,-0.7552665484,2.1897355098

H,0,-0.0095249553,-2.3086926297,-0.0305119602

C,0,-4.2582271234,0.0107803451,0.0073849996

Li,0,-6.598586497,0.0175558636,-0.0010189386

Li,0,-1.9678347087,0.0038751437,0.0109838588

Li,0,1.9696946653,-0.0143097727,-0.0014079556

C,0,4.2588763518,-0.0180341016,-0.0041023468

Li,0,6.5970892667,-0.0179763403,-0.006416525

C,0,8.5017318098,-1.1297276334,0.4038985333

C,0,8.495150805,-0.7280542286,-0.9718915674

C,0,8.4774477937,0.0548997802,1.2112346823

H,0,8.4918560039,-2.1507161673,0.7699056013

C,0,8.4667127005,0.7049935486,-1.0147991988

H,0,8.4788236639,-1.391851129,-1.8294969553

C,0,8.4556914307,1.1884224095,0.3342203575

H,0,8.4461420407,0.0874006985,2.2949075515

H,0,8.4245456658,1.3152527758,-1.9104208627

$\mathrm{H}, 0,8.4034357702,2.2282576372,0.6379286294$

Al,0,4.2961984575,0.2493533284,-2.0594562005

Al,0,4.299968941,-2.0720502226,-0.27242577

Al,0,4.3041423026,-0.284200744,2.0502314369

Al,0,4.3014868113,2.0375435422,0.2611067128

Al,0,-4.2936389386,1.6841782112,-1.213221372

Al,0,-4.2963540903,-1.2123368704,-1.6657301025

Al,0,-4.3063884758,-1.6646711115,1.2274266336

Al,0,-4.303749583,1.2282785549,1.6832103982

HF=-702.8817486 (B3LYP/LanL2DZ) 


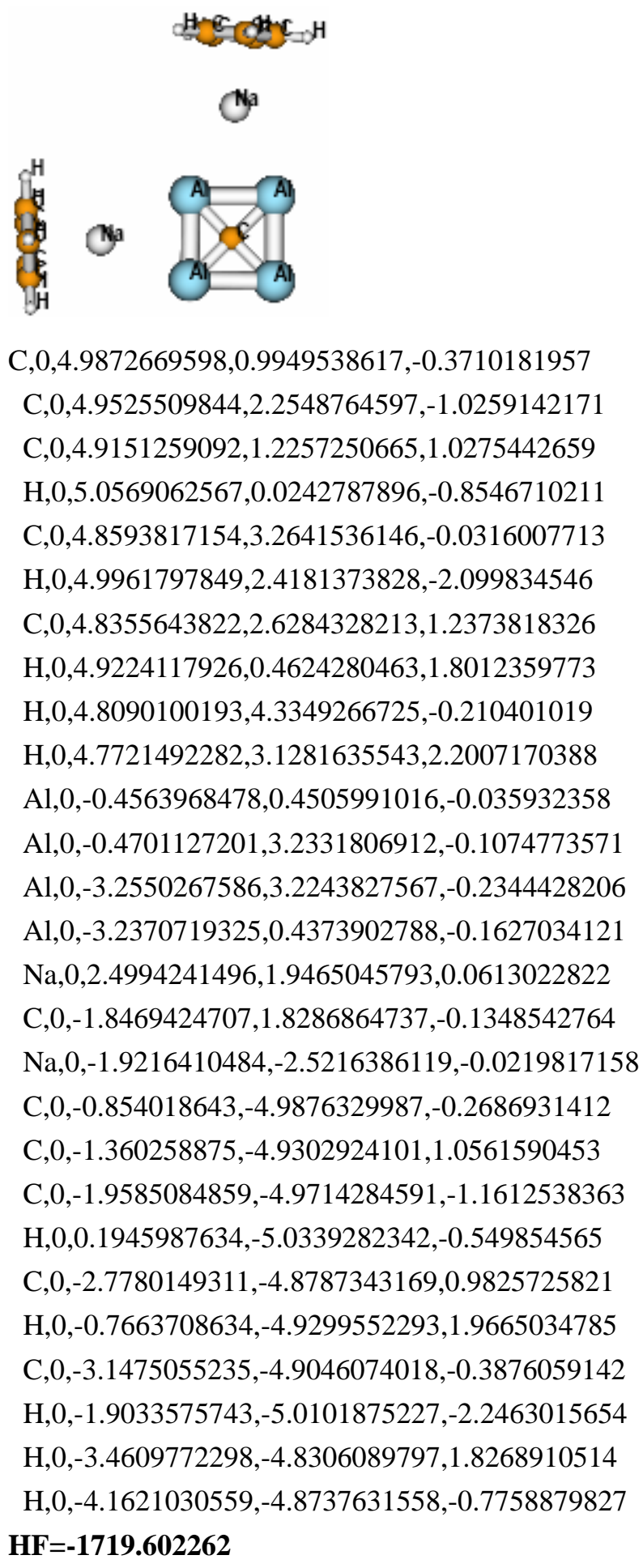




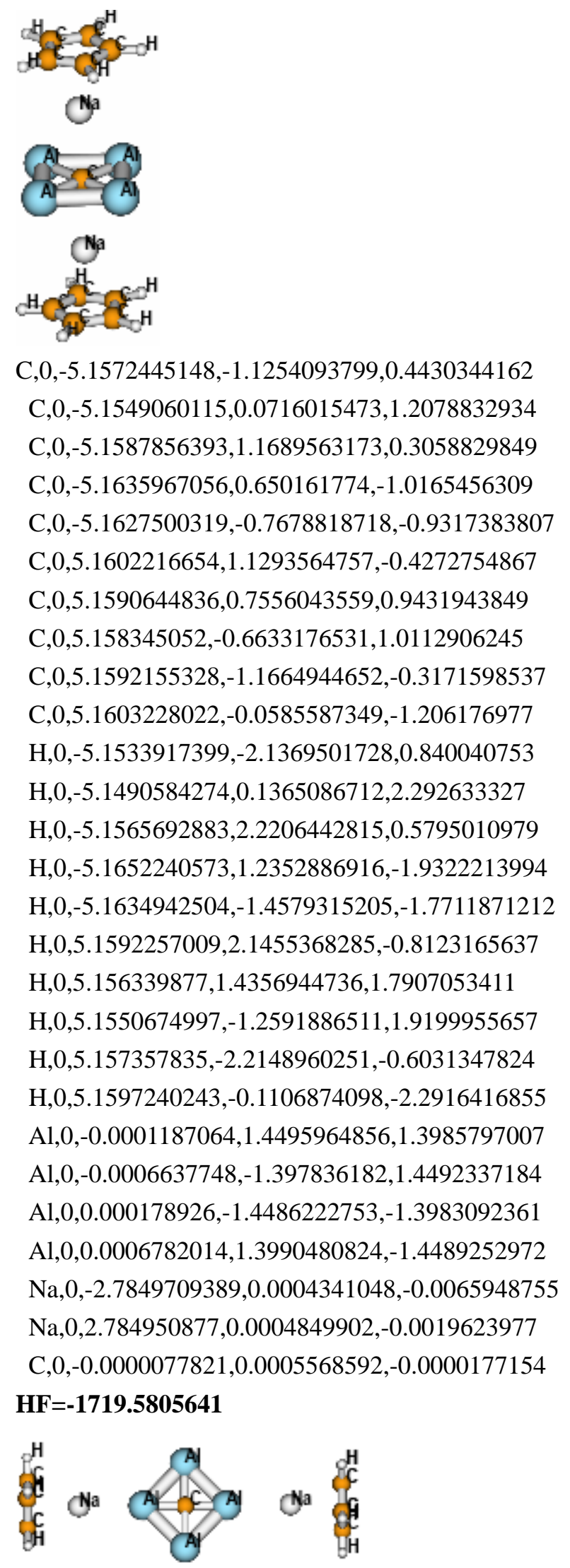

Al,0,1.4346224171,-1.3780926383,-0.0026922945 Al,0,1.3367535337,1.4047374748,-0.0000831545 Al,0,-1.4469255934,1.3897386131,0.0002001562 
Al,0,-1.3500742539,-1.3920784649,-0.0021896676

C, $0,-0.0060186456,0.0057119228,-0.0012841178$

$\mathrm{Na}, 0,3.4161897415,3.5666458481,-0.0018868275$

$\mathrm{Na}, 0,-3.4148843864,-3.5679082923,0.0016715736$

C,0,5.4305782229,4.9451205122,-1.1087983692

C,0,4.427058472,5.909991872,-0.8265872896

C,0,5.9379363805,4.4649095325,0.1275341814

H,0,5.7594205202,4.6355313573,-2.0974361766

C,0,4.3141121893,6.0258371112,0.5840877574

H,0,3.8514990877,6.4672061257,-1.5610665522

C,0,5.2476535887,5.1324129831,1.1739249343

H,0,6.7206621462,3.7211831817,0.2512205374

H,0,3.6367851825,6.6869273849,1.1182791675

$\mathrm{H}, 0,5.4123901399,4.9918657577,2.2390918061$

C,0,-4.4018318359,-5.9267195648,0.8110030771

C,0,-5.4054659657,-4.97038117,1.1204913353

C,0,-4.3095610905,-6.0241507783,-0.6025604194

H,0,-3.8126242222,-6.4903052489,1.5296236795

C,0,-5.9336871778,-4.4771199301,-0.1018951587

H,0,-5.7208323703,-4.6749266807,2.1178105315

C,0,-5.2561109095,-5.1279353841,-1.166916732

H,0,-3.6372880669,-6.6751472302,-1.1552197142

H,0,-6.721694674,-3.7357455126,-0.2043693303

H,0,-5.4375453656,-4.9747296891,-2.227616863

HF $=-\mathbf{1 7 1 9 . 5 9 8 0 3 7}$

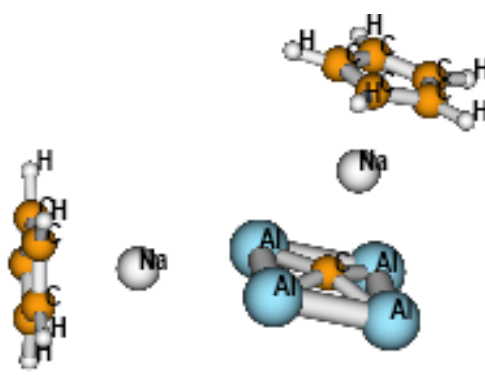

Al,0,0.7892825614,-1.1154742142,-1.3024544862 Al,0,-1.067641955,-3.1538086978,-1.6362809119 Al,0,-3.1564737105,-1.2720206724,-1.479498213 Al,0,-1.2940585242,0.7622411338,-1.144810547 C,0,-1.1869879259,-1.2248867775,-1.0894400404 C,0,2.4392316966,4.5682559728,-1.9401216776

C, $0,2.8762786841,4.3488493773,-0.6075622581$ C,0,3.2272351402,3.7571898104,-2.7993429114 H,0,1.6385233098,5.235679957,-2.247644851 C,0,3.9351501876,3.4024132518,-0.6429805519 $\mathrm{H}, 0,2.4724758483,4.8231972258,0.283175988$ C,0,4.1522795195,3.0373268947,-1.9974654043 
H,0,3.1404922479,3.7005420708,-3.8815798121 $\mathrm{H}, 0,4.4844595688,3.0255355111,0.2159522648$ H,0,4.8925221942,2.327241502,-2.3565642961 C,0,-1.9544060192,-0.8657170349,3.9630584692 C,0,-2.6901904866,-2.0574748585,3.726742027 C,0,-0.5745229303,-1.201085973,3.9968988745 H,0,-2.3734904879,0.1279120748,4.0976379476 C,0,-1.7651898329,-3.1294523961,3.6144169705 H,0,-3.7711413196,-2.1352070465,3.6457880774 C,0,-0.4576767364,-2.6002454975,3.7813047389 H,0,0.2470274731,-0.5094919646,4.1634280656 H,0,-2.013952472,-4.1715063359,3.4319948182 H,0,0.469438218,-3.166510642,3.7518787067 $\mathrm{Na}, 0,1.7234338213,2.0210615587,-1.3632770564$ Na,0,-1.3341584752,-1.5974812961,1.4707732133 HF $=-\mathbf{1 7 1 9 . 5 9 5 6 6 3 7}$

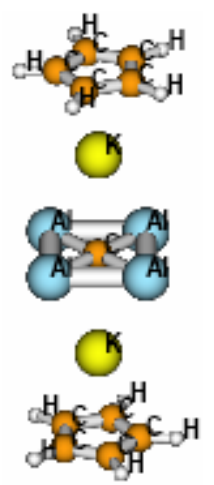

C,0,6.1534349238,1.2051500401,-0.0866238456 C,0,6.1518317542,0.4567022833,1.1194802291 C,0,6.1556973397,-0.9216285456,0.7803247786 C,0,6.1600781619,-1.0250749258,-0.6353801459 C,0,6.158513163,0.2894024173,-1.171191719 C,0,-6.1554962433,-1.2043935254,0.0857466837 C,0,-6.1549800128,-0.2905931547,1.1719626355 C,0,-6.1558175419,1.0248435438,0.638533656 C,0,-6.1565939716,0.9239565061,-0.7773561101 C,0,-6.1566398199,-0.4537691153,-1.1190161631 H,0,6.1664281759,2.2896889919,-0.1657799168 H,0,6.1628949457,0.8672403616,2.1264491281 H,0,6.1703031192,-1.752396898,1.4819268275 H,0,6.1788984953,-1.948979059,-1.2087068246 $\mathrm{H}, 0,6.176332236,0.5493196113,-2.2270308993$ H,0,-6.1704291054,-2.2890471,0.1630094472 H,0,-6.169502573,-0.5523743325,2.22738575 H,0,-6.1706802596,1.9477380284,1.2135863691 H,0,-6.1720337547,1.7560293285,-1.4773958069 
H,0,-6.1721745568,-0.8624578385,-2.1266868673 Al,0,0.0003769667,-0.8058302894,1.8302302458 Al,0,0.0005523543,1.8292047267,0.808659747 Al,0,-0.0003833594,0.8077300823,-1.8265140499 Al,0,-0.0005663429,-1.8275450414,-0.8048829616 $\mathrm{K}, 0,3.3375112519,-0.0029977098,-0.0057624681$ $\mathrm{K}, 0,-3.3375220065,-0.0013964469,-0.0023105401$ C,0,0.0000443423,0.0008135875,0.0017235326 HF $=-\mathbf{2 5 9 4 . 8 0 2 3 2 3 6}$

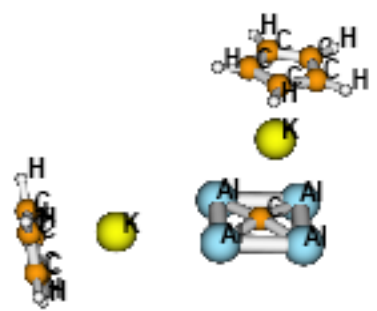

Al,0,1.9546737672,-0.3173533618,0.6422884093 Al,0,1.818394332,-1.2049830894,3.27893414 Al,0,-0.2684511216,-2.9628640062,2.579493504 $\mathrm{Al}, 0,-0.1329412769,-2.0748216051,-0.0573371212$ C,0,0.7204561549,-1.5050551802,1.6368456223 C,0,1.3805409197,-1.0392527606,-6.0771057226 C,0,0.8083867132,0.2557064984,-5.9794397135 C,0,2.7650512377,-0.9301986047,-5.7837495036 H,0,0.853937882,-1.9516850904,-6.3470950168 C,0,1.8392595673,1.165274054,-5.6256817586 H,0,-0.2327749551,0.5098239279,-6.1647764634 C,0,3.0484563377,0.4323905428,-5.5048316754 H,0,3.4864083034,-1.7442914284,-5.7924978315 $\mathrm{H}, 0,1.7267534685,2.238850471,-5.492501588$ H,0,4.0240655943,0.8453849794,-5.2591798566 C,0,-4.2034016773,1.9740125932,2.8084751428 C,0,-3.8016064216,1.8687414216,4.165833728 C,0,-3.4031448149,2.9700824154,2.1901167605 H,0,-4.9984332199,1.4057560831,2.3312850025 C,0,-2.7531711219,2.7997351495,4.3863671598 H,0,-4.2337624349,1.2043132935,4.9105129307 C,0,-2.5068591134,3.4804588681,3.1653240665 H,0,-3.4787766782,3.3000574656,1.1566474716 H,0,-2.2411712299, 2.9744030889,5.3298272175 H,0,-1.7742775699,4.2689871972,3.009702386 K,0,1.4208746612,-0.4740579163,-3.0358421853 K,0,-1.438114659,0.6528640658,2.6285382376 $\mathrm{HF}=-\mathbf{2 5 9 4 . 8 1 1 5 4 5 1}$ 


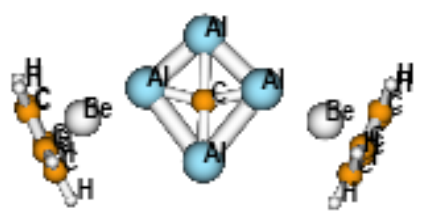

Al,0,-2.6925603197,-0.0802117231,-0.1134905041

Al,0,-0.9081810766,-1.8759753145,-0.2740306316

Al,0,1.3960645959,0.0414285098,0.0593254885

Al,0,-1.037794352,1.8178805807,0.1924126888

C,0,-0.5511866067,-0.0164953634,-0.0229619868

Be,0,0.1687430681,-3.8822149948,-0.4945971421

Be,0,-0.1045006832,3.8841188803,0.4973655027

C,0,-0.0803582738,-5.584187939,-1.3435585301

C,0,1.1018681636,-4.9322994029,-1.795920699

C,0,-0.000258902,-5.7067182749,0.0702420626

H,0,-0.9240264718,-5.8688817654,-1.9587812872

C,0,1.914352612,-4.6563321996,-0.6613185098

H,0,1.3154619073,-4.6393908635,-2.8156915835

C,0,1.2323367804,-5.1316411001,0.4923539217

H,0,-0.7713330288,-6.1031101754,0.7180774186

H,0,2.8457241309,-4.1054964107,-0.6655811996

H,0,1.5620172598,-5.0163015987,1.5166814983

C,0,-0.525630913,5.5668156229,1.3162972363

C,0,0.6503718118,4.9855776993,1.8698589169

C,0,-0.3327978157,5.6962986951,-0.0859576268

$\mathrm{H}, 0,-1.434054723,5.7997954311,1.8564936783$

C,0,1.5715067886,4.7603351121,0.8094825109

$\mathrm{H}, 0,0.7933281051,4.7041442384,2.9050938882$

C,0,0.9632395204,5.1962350647,-0.3996566624

H,0,-1.0680511018,6.0472526162,-0.7984218314

$\mathrm{H}, 0,2.5301871149,4.2658320482,0.8948715587$

H,0,1.3852432564,5.1024267639,-1.391800924

\section{$\mathrm{HF}=\mathbf{- 1 4 2 4 . 4 6 5 1 6 7 2}$}

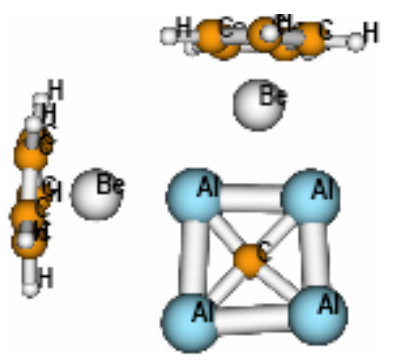

C,0,3.7782154668,-0.243173886,0.1261671503

C,0,3.7570237186,0.9045817678,-0.7151155433

C,0,3.562801799,0.1888673475,1.463184134

H,0,3.876494417,-1.2697332802,-0.2026142231

C,0,3.5343884587,2.046131551,0.1036317762 
H,0,3.8354774341,0.9037676834,-1.7946060965

C,0,3.4115481171,1.6043848964,1.4488818791

H,0,3.4706025075,-0.4501979631,2.3319303569

H,0,3.3974694063,3.0614459309,-0.2440365355

H,0,3.1756177831,2.22817916,2.3011467755

Al,0,-0.1379185406,0.4265326045,-0.0033720968

Al,0,-0.5115232026,3.3052799838,-0.11832137

Al,0,-3.3285908287,2.6451109924,-0.5249166592

Al,0,-2.7286028875,0.0198906323,-0.3921381651

Be,0,2.1360696633,0.7477981087,0.3019413095

C,0,-1.582775753,1.6555704672,-0.2429618722

Be,0,-1.1775772459,-1.9266143058,-0.1292371406

C,0,0.0030965301,-3.3148355514,-0.8158114604

C,0,0.0402884497,-3.2644242027,0.6026249811

C,0,-1.3469960606,-3.52904041,-1.2127180049

H,0,0.842028075,-3.1560875915,-1.4809082142

C,0,-1.2875183492,-3.4395581339,1.0833533385

H,0,0.911727729,-3.0539083268,1.2083882658

C,0,-2.1409476966,-3.6159470048,-0.0389050731

H,0,-1.7131871344,-3.5612095297,-2.2306475609

H,0,-1.6001031723,-3.3972521055,2.1188240012

H,0,-3.2185838287,-3.7036650027,-0.0085534933

$H F=-1424.4612476$

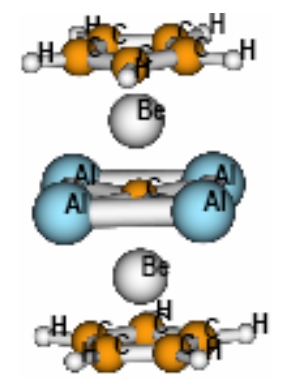

C,0,3.2117355475,0.0111754792,1.2103811373

C,0,3.2019979788,-1.1443656364,0.3735552089

C,0,3.2116884114,-0.7044250431,-0.9836211771

C,0,3.2273634353,0.7208118744,-0.9840898551

C,0,3.2275192122,1.1626116121,0.3703990645

C,0,-3.2161358776,-0.00557342,-1.2120472583

C,0,-3.2232020862,-1.1576856804,-0.3728584229

C,0,-3.2226657082,-0.7171442295,0.9816234724

C,0,-3.2154036242,0.708150579,0.9824943672

C,0,-3.2118870377,1.1493377064,-0.3743256031

H,0,3.1739786145,0.0156569376,2.2914852673

H,0,3.1513583122,-2.1716327055,0.7086132953

H,0,3.1738607862,-1.338346532,-1.8593820051

H,0,3.2139113066,1.3575977078,-1.8584304737 
H,0,3.213616366,2.1925241027,0.7007233171

H,0,-3.180912434,-0.0091232064,-2.2932455242

H,0,-3.2033555104,-2.1871183187,-0.7044905595

$\mathrm{H}, 0,-3.2026671727,-1.3542639821,1.8556488751$

H,0,-3.1795005542,1.3414014151,1.8588279373

H,0,-3.1683392524,2.1771615922,-0.7086302099 Al,0,0.0122949991,-1.905928655,-0.9673176033 Al,0,0.0107485818,-0.9839402384,1.8972942366 Al,0,-0.0098214186,1.900481815,0.9866428623 Al,0,-0.0077327986,0.9694099536,-1.9092600324 Be,0,1.7150194429,0.019854541,-0.0064958191 Be,0,-1.7165240328,-0.0014394865,-0.0003427576 C,0,-0.0005592229,0.0041376573,-0.0014173728

\section{$\mathrm{HF}=\mathbf{- 1 4 2 4 . 4 5 2 8 1 8 4}$}

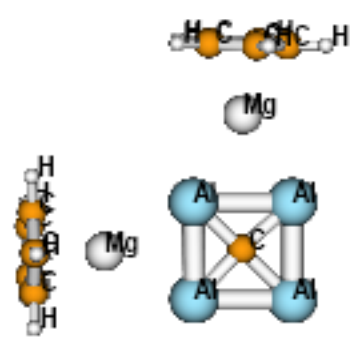

C,0,4.3148996812,0.6760878368,-0.4582448314

C,0,4.2999336082,1.9787433774,-1.0384460114 C,0,4.2431742055,0.8229772327,0.9546988143 H,0,4.3893665276,-0.2607378289,-0.9982231366 C,0,4.2251856051,2.9303197014,0.0188310332 H,0,4.3706947996,2.2061559835,-2.0960029499 C,0,4.1847971212,2.217196231,1.2507496146 H,0,4.2548705238,0.0177923995,1.6802772831 H,0,4.2110150308,4.0080006518,-0.0937783743 H,0,4.1518761292,2.6576318607,2.2407146059 Al,0,-0.2737516963,0.2680720227,-0.0151249254 Al,0,-0.2983623985,3.0492032877,-0.1412343879 Al,0,-3.0610867451,3.037659239,-0.2857187806 Al,0,-3.0529564719,0.2751091327,-0.1613049069 Mg,0,2.2196759195,1.6758401559,0.0490932306 C,0,-1.6333388271,1.6199306563,-0.1467251346 Mg,0,-1.6725055649,-2.235123705,0.0174083793 C,0,-0.5050829283,-4.2945955652,-0.2122019069 C,0,-1.0365603559,-4.2457804156,1.1066905244 C,0,-1.597289795,-4.3066699664,-1.1275610028 H, 0,0.5460180288,-4.3293307778,-0.4741968476 C,0,-2.4598904753,-4.2326005139,1.008360067 H,0,-0.4619006535,-4.2483397011,2.0258270311 


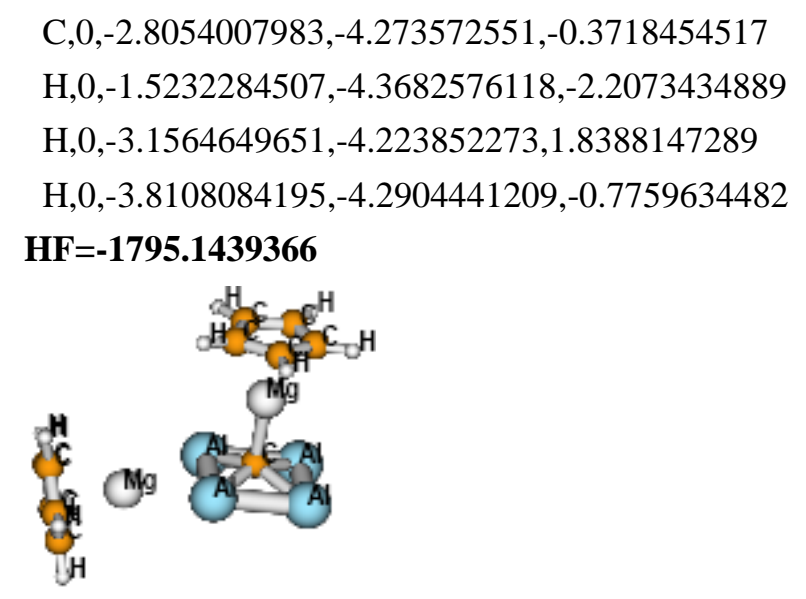

Al,0,0.9678862334,-1.0744964931,-1.0259619131 Al,0,-0.9368059645,-2.9853769004,-1.5233452087 Al,0,-2.9526547854,-1.0788450357,-1.5014735648 Al,0,-1.1443500197,0.9241557055,-1.0034936832 C,0,-1.0117191488,-1.0543376238,-0.7127545451 C,0,2.1474818787,4.0459230034,-1.2429904644 C,0,2.8868871701,3.4448516974,-0.1848656593 C,0,2.6051255848,3.4897480308,-2.4705579044 $\mathrm{H}, 0,1.3772897879,4.8005036081,-1.1338449197$ C,0,3.8097256237,2.5225886096,-0.7605170671 H,0,2.7920323607,3.6769140026,0.8699153407 C,0,3.6353366241,2.5492878596,-2.1714344923 H,0,2.2562796387,3.759150254,-3.4609738696 H,0,4.5304553773,1.9204972939,-0.2193920405 H,0,4.2010033824,1.9722736586,-2.8938917226 C,0,-2.3867095947,-0.8174131526,3.3270896512 C,0,-2.4761906877,-2.2274070488,3.1332077802 C,0,-1.0126694683,-0.4847539155,3.513459394 H,0,-3.2200477293,-0.1251211965,3.3595765811 C,0,-1.1572810986,-2.7658572947,3.1993505115 H,0,-3.3893335988,-2.7942942461,2.9930362739 C,0,-0.2529784735,-1.6887922691,3.4345064554 $\mathrm{H}, 0,-0.6189718862,0.5049736326,3.7146194442$ H,0,-0.8925413479,-3.8137371735,3.1181530114 H,0,0.8196574915,-1.7753849364,3.5639726048 Mg,0,1.6143464315,1.7248020549,-1.229312072 Mg,0,-1.2577510119,-1.3430926272,1.3465982509 HF $=-\mathbf{1 7 9 5 . 1 3 7 8 0 1 1}$ 


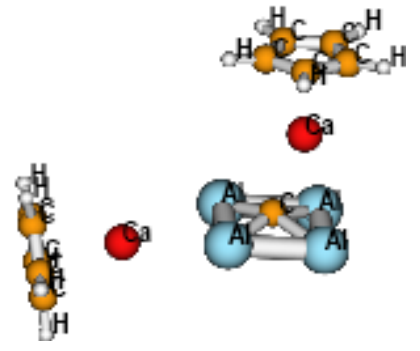

Al,0,-0.4035252261,1.0836034091,1.4347906

Al,0,1.7986430711,2.7168260082,1.4452691113

Al,0,1.8228380591,2.7439041445,-1.3790701717

Al,0,-0.3839807951,1.1163295694,-1.4363975449

C,0,0.9081345245,1.5295883722,0.0127684286

C,0,-5.3577491503,-0.9228019728,-1.267267765

C,0,-4.8875903209,-2.0125369309,-0.4830262569

C,0,-5.814103462,0.0920529757,-0.3807514784

H,0,-5.41642468,-0.8959715806,-2.3505361032

C,0,-5.0543244344,-1.6716003273,0.8887227184

H,0,-4.5315781326,-2.9643149731,-0.8645282289

C,0,-5.6265238315,-0.3712010849,0.9520070239

H,0,-6.2890320279,1.0240418065,-0.6705277704

H,0,-4.8438238352,-2.31599399,1.7362365109

H,0,-5.9283942315,0.1481614104,1.8558507134

C,0,4.1524597733,-2.1687067353,-1.1596398387

C,0,5.1796954624,-1.4916099261,-0.4444916623

C,0,3.2553653649,-2.73385517355,-0.2111702492

H,0,4.1019972947,-2.2932050899,-2.2365739719

C,0,4.9170850136,-1.6386367631,0.9464956729

H,0,6.0493639456,-1.0105852421,-0.8805088298

C,0,3.7280986633,-2.4067658982,1.0901755583

H,0,2.4020711642,-3.36446044,-0.4396153555

H,0,5.5505183991,-1.2879410854,1.7549323453

H,0,3.2967799852,-2.7421696813,2.0278626211

Ca,0,-3.1234190956,0.0024374451,-0.0314444236

Ca,0,2.9415970999,-0.0579255292,0.009683385

HF $=-\mathbf{2 7 5 0 . 0 8 6 9 8 1 4}$

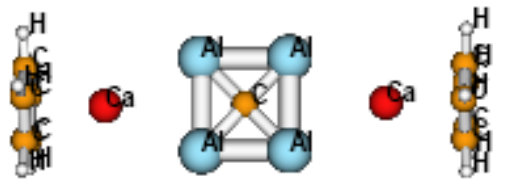

Al,0,-1.3455134779,-1.4305284846,0.0061409241

Al,0,1.3539885982,-1.4228042967,0.0149845707

Al,0,1.3448484597,1.4111076575,0.0901025501

Al,0,-1.3533494041,1.4031381285,0.081210684

C,0,0.000010303,-0.0102103705,0.0538893235 
Сa,0,4.2866368277,0.005978326,0.0274195687

Ca,0,-4.2865348785,-0.018380072,0.0039765628

C,0,6.6928777967,-0.4176367608,-1.1593671979

C,0,6.685677891,0.9632077972,-0.8147839236

C,0,6.7349132515,-1.1713747205,0.0462371521

H,0,6.7300818106,-0.8234936811,-2.1653391686

C,0,6.7230775631,1.0625244928,0.6033885646

H,0,6.7122002998,1.7945489179,-1.5119163868

C,0,6.7532911319,-0.2569129696,1.136038372

H,0,6.8046836126,-2.2518290173,0.1198528324

H,0,6.7828697519,1.982616957,1.1757875789

H,0,6.8471428821,-0.5185723102,2.1852800807

C,0,-6.7293769168,-1.1800695369,0.2591585681

C,0,-6.7493108739,-0.0689907427,1.1479467648

C,0,-6.6933648937,-0.6780905029,-1.0711307446

H,0,-6.7938923844,-2.2252970856,0.5438108091

C,0,-6.726420869,1.1198645852,0.3662731887

H,0,-6.8395835304,-0.119244815,2.2284842301

C,0,-6.6916229189,0.7436511064,-1.0048445582

H,0,-6.7299943492,-1.2741089308,-1.9774586247

H,0,-6.7878680407,2.1342966831,0.7468564075

H,0,-6.7258521112,1.4214748747,-1.8518169213

HF $=-\mathbf{2 7 5 0 . 1 0 6 8 7 2 6}$ 